Aus der Klinik für Psychiatrie und Psychotherapie

(Prof. Dr. med. J. Wiltfang)

der Medizinischen Fakultät der Universität Göttingen

\title{
Pharmakologische Behandlung von stationären Patienten mit einer emotional instabilen Persönlichkeitsstörung
}

\author{
INAUGURAL-DISSERTATION \\ zur Erlangung des Doktorgrades \\ für Zahnheilkunde \\ der Medizinischen Fakultät der \\ Georg-August-Universität zu Göttingen
}

\author{
vorgelegt von \\ Margarita Nuss \\ aus \\ Zelinograd
}

Göttingen 2015 
Dekan:

I. Berichterstatter: $\quad$ PD Dr. med. D. Wedekind

II. Berichterstatter/in: Prof. Dr. med. C. Spitzer

III. Berichterstatter/in: Prof. Dr. med. dent. R. Mausberg

Tag der mündlichen Prüfung: 05.04.2016 


\section{Inhalt}

Abkürzungsverzeichnis

1 Einleitung 1

$1.1 \quad$ Konzept von Persönlichkeitsstörungen ........................................... 1

1.2 Historische Begriffsbestimmung der Borderline- Persönlichkeitsstörung ..... 2

1.3 Der Begriff der Borderline-Persönlichkeitsstörung.................................... 4

1.4 Definition der BPS nach ICD-10 und DSM-IV ........................................ 5

1.4.1 ICD-10-Kriterien für die Borderline-Persönlichkeitsstörung (F60.31; WHO 1993)

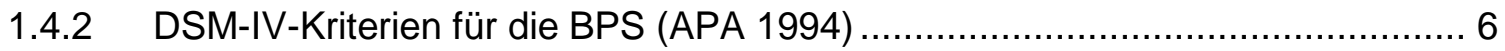

1.5 Diagnostik der Borderline-Persönlichkeitsstörung …................................ 7

1.6 Das klinische Erscheinungsbild der BPS ................................................ 8

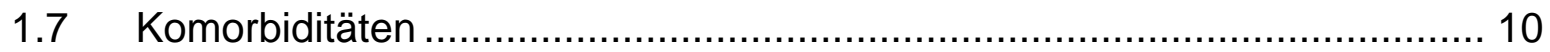

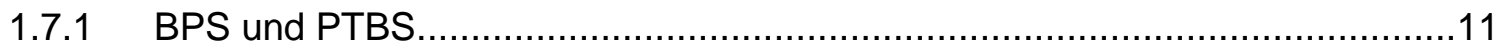

1.7.2 Epidemiologie der BPS ……………………....................................11

1.7.3 BPS und Suizidalität...........................................................................12

1.8 Pathogenese der Borderline-Persönlichkeitsstörung.............................. 13



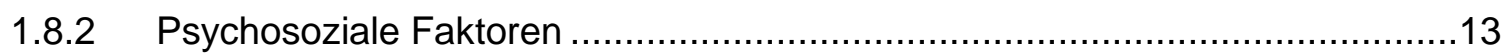

1.9 Der Verlauf und die Prognose bei BPS ……........................................ 16

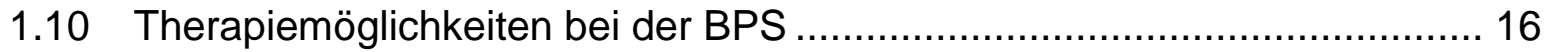

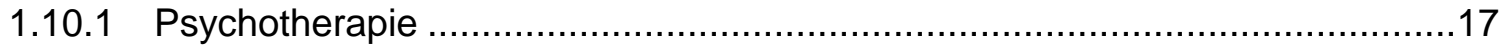

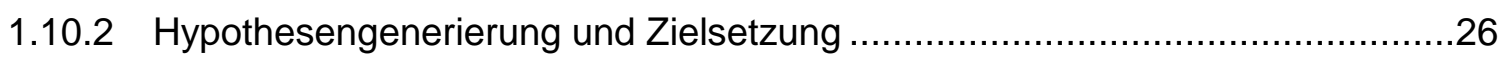

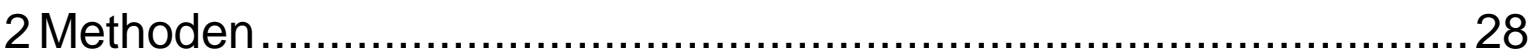

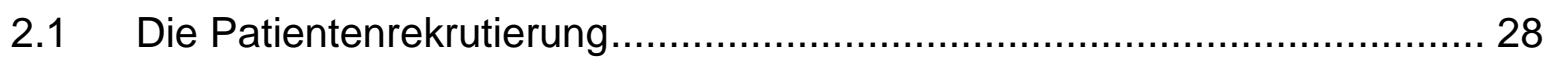

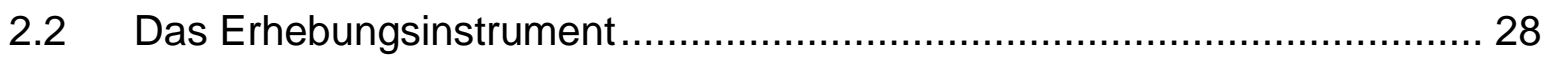

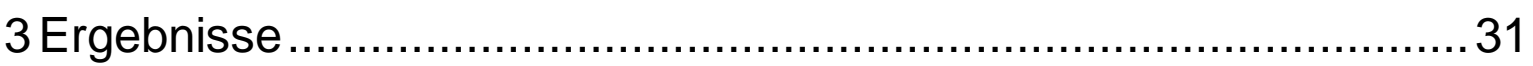

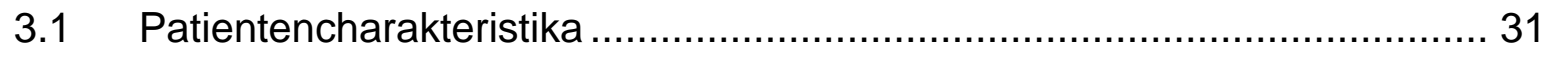

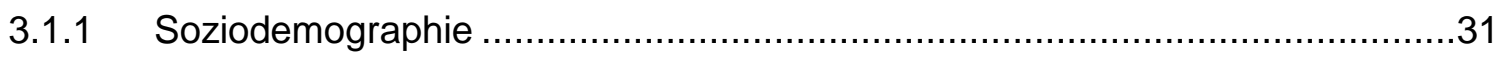

3.1.2 Psychiatrische Komorbiditäten .................................................................33

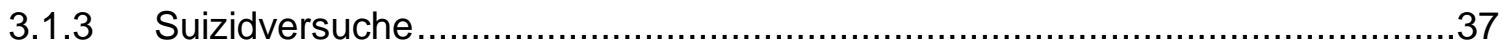

3.1.4 Dokumentation der diagnostischen Kriterien nach ICD-10 und DSM-IV ..........37 


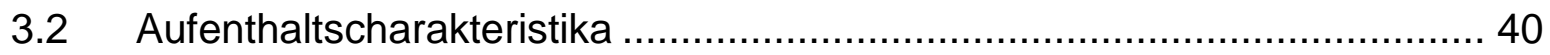

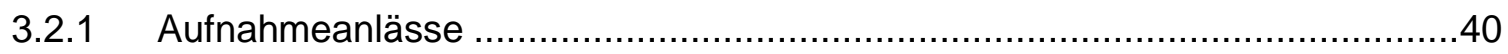

3.2.2 Dauer der stationären Behandlung bei verschiedenen Behandlungsmodi .......41

3.3 Psychopharmakotherapie ..................................................... 41

3.3.1 Psychopharmakaanamnese über den gesamten Behandlungszeitraum .........41

3.3.2 Häufigkeitsverteilung verschiedener Substanzgruppen ...............................42

3.4 Vergleich der Ergebnisse mit der Studie des Zeitraums 1996 - 2004........ 50

3.4.1 Vergleich Soziodemographie................................................................. 51

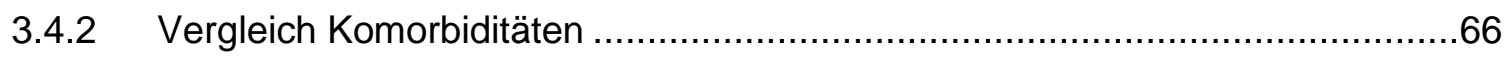

3.4.3 Vergleich der Dokumentation der diagnostischen Kriterien nach ICD-10.

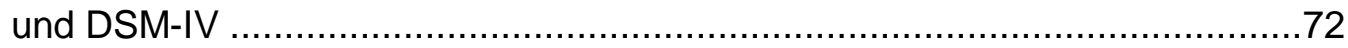

3.4.4 Veränderung der Psychopharmakotherapie zum Zeitpunkt der Entlassung.....77

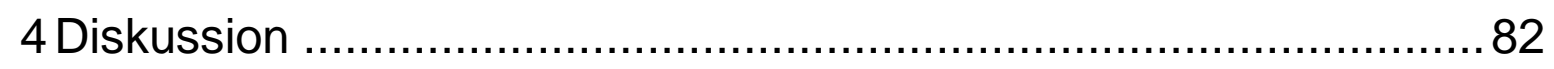

4.1 Psychopharmakotherapie allgemein .......................................... 83

4.2 Psychopharmakotherapie speziell ............................................... 85

4.3 Demographische Gesichtspunkte............................................ 91

4.4 Einschränkungen der Untersuchung .......................................... 94

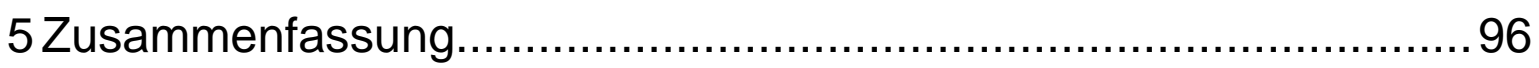

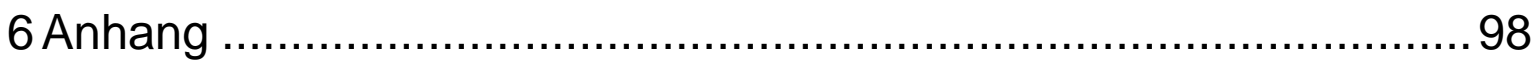

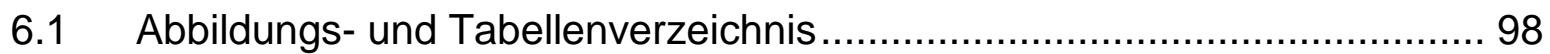

6.2 Das Erhebungsinstrument - der Fragebogen .................................. 101

7 Literaturverzeichnis ................................................... 110 


\section{Abkürzungsverzeichnis}

Abb.

Abbildung

APA

American Psychiatric Association

BPS

Borderline-Persönlichkeitsstörung

DBT

Dialektisch-Behaviorale Therapie

CBZ

Carbamazepin

DIB

Diagnostisches Interview für das Borderlinesyndrom

DSM

Diagnostic and Statistical Manual of Mental Disorders

GABA

ү-Aminobuttersäure

ICD

International Classification of Diseases

$\mathrm{MAOH}$

Monoaminooxidasehemmer

NL

Neuroleptika

n.e.

nicht eruierbar

n.s.

nicht signifikant

PS

Persönlichkeitsstörung

PSOM

Psychosomatik

PSY

Psychiatrie

SD

Standardabweichung

SSRI

selektiver Serotonin-Wiederaufnahme-Hemmer

tZA

Trizyklisches Antidepressivum

Uni-Gö

Universität Göttingen

VPA

Valproat

WHO

Weltgesundheitsorganisation 


\section{Einleitung}

\subsection{Konzept von Persönlichkeitsstörungen}

Persönlichkeitsstörungen sind gekennzeichnet durch tief verwurzelte, von der jeweiligen Kultur abweichende Verhaltensmuster, Einstellungen, Gedanken und Wahrnehmungen.

Das überdauernde Muster ist unflexibel und sozial wenig angepasst, es äußert sich in starren Reaktionen auf unterschiedliche persönliche und soziale Lebenslagen. Es resultiert eine komplexe Beziehungs- und Anpassungsstörung der Betroffenen an die Umwelt.

Persönlichkeitsstörungen gehen häufig mit persönlichem Leid und gestörter sozialer Funktions- und Leistungsfähigkeit einher. Nicht selten sind sie aber auch durch eine geringere bis fehlende Störungseinsicht gekennzeichnet, so dass vorrangig das Umfeld leidet.

Die Auffälligkeiten der Persönlichkeitsstruktur sind stabil und lang andauernd. Sie beginnen in der Kindheit oder frühen Adoleszenz und dauern bis ins späte Erwachsenalter oder lebenslang.

Pathogenetisch lassen sich oft psychosozial ungünstige Bedingungen in der Entwicklung finden, worauf später detaillierter eingegangen wird.

Im Gegensatz hierzu muss man „Persönlichkeitsänderungen“ oder anhaltende Persönlichkeitsänderungen nach Extrembelastungen (ICD-10, F62.0) abgrenzen. Diese werden im Erwachsenalter erworben, in Folge schwerer oder anhaltender Belastungen, extremer, umweltbedingter Deprivation, schwerwiegender psychiatrischer Störungen, Hirnerkrankungen oder -verletzungen (Schmitz et al. 1996).

Auf der folgenden Seite findet sich ein tabellarischer Überblick über die verschiedenen Persönlichkeitsstörungen (Tabelle 1). 
Tabelle 1 Übersicht über die verschiedenen Persönlichkeitsstörungen in der ICD-10 und dem DSM-IV

(International Classification of Diseases, zehnte Version; WHO 1993) und dem DSMIV (Diagnostic and Statistical Manual of Mental Disorders, vierte Version; APA 1994)

\begin{tabular}{|c|c|}
\hline ICD-10 & DSM-IV \\
\hline $\begin{array}{l}\text { Paranoide PS (F60.0) } \\
\text { Schizoide PS (F60.1) }\end{array}$ & $\begin{array}{l}\text { Cluster A (sonderbar, exzentrisch) } \\
\text { Paranoide PS (301.00) } \\
\text { Schizoide PS (301.20) } \\
\text { Schizotypische PS (301.22) }\end{array}$ \\
\hline $\begin{array}{l}\text { Dissoziale PS (F60.2) } \\
\text { Emotional instabile PS (F60.3) } \\
\text { impulsiver Typus (F60.30) } \\
\text { Borderline-Typus (F60.31) } \\
\text { Histrionische PS (F60.4) }\end{array}$ & $\begin{array}{l}\text { Cluster B (dramatisch, emotional, launisch) } \\
\text { Antisoziale PS (301.7) } \\
\text { Borderline PS (301.83) } \\
\text { Histrionische PS (301.50) } \\
\text { Narzisstische PS (301.81) }\end{array}$ \\
\hline $\begin{array}{l}\text { Anankastische PS (F60.5) } \\
\text { Ängstlich-vermeidende PS (F60.6) } \\
\text { Abhängige PS (F60.7) } \\
\text { sonstige näher bezeichnete PS (F60.8) } \\
\text { nicht näher bezeichnete PS (F60.9) }\end{array}$ & $\begin{array}{l}\text { Cluster C (ängstlich) } \\
\text { Zwanghafte PS (301.4) } \\
\text { Selbstunsichere PS (301.82) } \\
\text { Dependente PS (301.6) } \\
\text { Nicht näher bezeichnete PS (301.9) }\end{array}$ \\
\hline
\end{tabular}

PS = Persönlichkeitsstörung (modifiziert nach Saß 2000; Schmitz et al. 1996)

Die vorliegende Arbeit befasst sich ausschließlich mit der emotional instabilen Persönlichkeitsstörung bzw. der Borderline-Persönlichkeitsstörung

\subsection{Historische Begriffsbestimmung der Borderline- Persönlichkeitsstörung}

Das wissenschaftliche Interesse für Persönlichkeitsstörungen beginnt mit dem französischen Psychiater Philippe Pinel (1745-1826), der als erster den Versuch unternahm, die Störung der Persönlichkeit als nosologische Einheit herauszuarbeiten (Saß et al. 2002). Pinel und Esquirol (Jean-Etienne Dominique Esquirol, 1772-1840, 
franz. Psychiater) beobachteten Auffälligkeiten, die heute als charakteristisch für PS zählen, z.B. Veränderungen des Willens und der Gefühle bei unbeeinträchtigter Intelligenz ( $\mathrm{Saß}$ et al. 2002). Der amerikanische Arzt Benjamin Rush (1745-1813) bezog sich auf Pinels Konzept der manie sans délire (1809) und beschrieb mit dem Begriff der moral alienation of the mind (1812) Personen, die bei unauffälliger Intelligenz antisoziales Verhalten zeigten ( $\mathrm{Saß}$ et al. 2002). Partridge (1930) führte später den Begriff sociopathy für psychopathische Persönlichkeiten ein, der im angelsächsischen Raum als Synonym für psychopathy eingesetzt wurde (Saß et al. 2002). In Deutschland wurde der Begriff „Psychopathie“ zunächst als Oberbegriff für alle psychischen Abnormitäten verwendet und erst ab 1883 entwickelte der deutsche Psychiater Emil Kraepelin (1856-1926) das Konzept der psychopathischen Zustände im Sinne des heutigen Verständnisses von „abnormen Persönlichkeiten“. 1923 unternahm Kurt Schneider (deutsche Psychiater, 1887-1967) durch seine Monographie „Die psychopathischen Persönlichkeiten“ einen Versuch, durch deskriptiv-symptomatologische Beschreibungen einen wertneutralen psychopathologischen Standpunkt einzunehmen. $\mathrm{Er}$ führte in die Definition von Persönlichkeitsstörungen die Orientierung an eine statistische Norm und den durch die Störung verursachten Leidensdruck für den Betroffenen und/oder das Umfeld ein (Saß, et al. 2002). Diese Aspekte finden sich auch in der heutigen Definition von Persönlichkeitsstörungen wieder. 1974 löste der Begriff der PS den der „Psychopathie“ ab, der in den modernen internationalen Klassifikationsschemata der WHO nicht mehr zu finden ist.

Im Gegensatz zu den aufgegebenen Begriffen der „Psychopathie“ oder der "Charakterneurose" nimmt der Begriff der PS einen ätiologiefreien und rein beschreibenden Standpunkt ein.

Nach dem heutigen Verständnis werden Persönlichkeitsstörungen als "charakteristische, dauerhafte innere Erfahrungs- oder Verhaltensmuster" definiert (Bronisch 2003), die deutlich von den kulturell erwarteten Normen abweichen. Sie gehen mit einem Leidensdruck bei den Betroffenen selbst sowie mit einem nachteiligen Einfluss auf die soziale Umwelt der Betroffenen einher.

Im ICD-10 (Internationale Klassifikation psychischer Störungen, Dilling et al. 2005) sind die Persönlichkeitsstörungen im Abschnitt „Persönlichkeits- und Verhaltensstörungen“ unter den Diagnoseziffern F60-F62 dargestellt. Sie werden beschrieben als „tief 
verwurzelte, anhaltende Verhaltensmuster, die sich in starren Reaktionen auf unterschiedliche persönliche und soziale Lebenslagen zeigen. Dabei findet man bei Personen mit PS gegenüber der Mehrheit der Bevölkerung deutliche Abweichungen im Wahrnehmen, Denken, Fühlen und in Beziehungen zu anderen. Solche Verhaltensmuster sind meistens stabil und beziehen sich auf vielfältige Bereiche von Verhalten und psychischen Funktionen. Häufig gehen sie mit persönlichem Leiden und gestörter sozialer Funktions- und Leistungsfähigkeit einher" (ICD-10, Dilling et al., S. 244). Im amerikanischen Klassifikationssystem DSM-IV (Diagnostical and Statistical Manual for Mental Disorders - IV, 1994) sind die Persönlichkeitsstörungen wie erstmals im DSM-III (APA 1980) zusammen mit den Entwicklungsstörungen mit einer eigenen Achse repräsentiert und werden in drei Hauptgruppen unterteilt. Das Cluster A beinhaltet die paranoide, schizotypische und schizoide PS, die durch sonderbare und exzentrische Verhaltensweisen auffallen. Das Cluster B fasst die antisoziale, emotional instabile, histrionische und narzisstische PS zusammen. Ihnen ist gemeinsam, dass die Betroffenen oft als dramatisch, emotional und launisch wahrgenommen werden. Das Cluster $\mathrm{C}$ beinhaltet die ängstliche, abhängige, selbstunsichere, anakastische und passiv-aggressive PS, die sich vor allem durch Ängstlichkeit kennzeichnen. Als Untergruppe der emotional instabilen Persönlichkeitsstörungen zählt die Borderline-Persönlichkeitsstörung zum Cluster B. Die Definition und eine nähere Beschreibung des klinischen Erscheinungsbildes der Borderline- Persönlichkeitsstörung folgen in den nächsten Abschnitten.

\subsection{Der Begriff der Borderline-Persönlichkeitsstörung}

Der Begriff der Borderline-Persönlichkeitsstörung (BPS) wurde durch Vertreter der psychoanalytischen Schule eingeführt und bezeichnete ursprünglich den Bereich zwischen neurotischen und psychotischen Erkrankungen. Lange Zeit stellte die BPS eine Restkategorie für schwer fassbare und kaum therapierbare Patienten dar (Herpertz und Wenning, 2002). Durch die Operationalisierung der Diagnosekriterien und durch die Einführung der Persönlichkeitsstörungen in die Klassifikationssysteme (erstmals 1980 im DSM-III) hat sich das geändert. Die Diagnosekriterien im DSM-III fundierten zum Teil auf der Arbeit von Gunderson und Singer (1975), die nach einer Durchsicht der BPS-Literatur jene Merkmale herausarbeitet haben, die es 
ermöglichen, beim Erstkontakt eine Diagnose zu stellen. Es handelte sich hierbei um die folgenden Merkmale: (1) einen intensiven depressiven oder feindseligen Affekt, (2) ein impulsives Verhalten, (3) eine begrenzte soziale Anpassungsfähigkeit, (4) ein Beziehungsverhalten, welches zwischen Oberflächlichkeit und Abhängigkeit schwankt, (5) psychosenahe Wahrnehmungen und (6) ein bizarres Denken in unstrukturierten Situationen. Zusammen mit dem von Kernberg entwickelten Kriterium der „Identitätsstörung“ wurden die oben erwähnten Merkmale in die DSM-III Definition der BPS aufgenommen (Skodol et al. 2002). Im DSM-IV kam noch die „Dissoziativität und Paranoia“ hinzu. Die Definition der BPS auf der Basis dieser neuen Kriterien, von denen mindestens fünf erfüllt sein müssen, führt zu einer Heterogenität des Krankheitsbildes, die die klinische Einordnung erschwert. Das alternative Klassifikationssystem der Weltgesundheitsorganisation (WHO) versucht dieser Problematik entgegenzusteuern, indem es, je nach Anzahl der erfüllten Kriterien, zwei Typen der emotional-instabilen PS unterscheidet. Trotzdem bleibt die Diagnose der BPS weiterhin verwirrend, da zwischen den Klassifikationssystemen bedeutsame Unterschiede vorliegen (Herpertz und Saß 2002). So steht im ICD-10 die Impulsivität im Mittelpunkt der diagnostischen Kriterien, im DSM-IV dagegen steht die Instabilität von Affekt, Verhalten und Beziehungsgestaltung im Mittelpunkt der Diagnose. Des Weiteren werden im ICD-10 die dissoziativen oder paranoiden Erlebnisse nicht erwähnt. Das DSM-IV hingegen unterscheidet nicht zwischen dem impulsiven Typ und dem Borderline Typus (Herpertz und Saß 2002).

\subsection{Definition der BPS nach ICD-10 und DSM-IV}

\subsubsection{ICD-10-Kriterien für die Borderline-Persönlichkeitsstörung (F60.31; WHO 1993)}

Mindestens drei der folgenden Eigenschaften oder Verhaltensweisen müssen vorliegen:

- Deutliche Tendenz, unerwartet und ohne Berücksichtigung der Konsequenzen zu handeln

- Deutliche Tendenz zu Streitereien und Konflikten mit anderen, vor allem dann, wenn impulsive Handlungen unterbunden oder getadelt werden 
- Neigung zu Ausbrüchen von Wut oder Gewalt mit Unfähigkeit zur Kontrolle explosiblen Verhaltens

- Schwierigkeiten in der Beibehaltung von Handlungen, die nicht unmittelbar belohnt werden

- Unberechenbare und unbeständige Stimmung.

Zusätzlich müssen mindestens zwei der folgenden Eigenschaften und Verhaltensweisen vorliegen:

- Störung und Unsicherheit bezüglich Selbstbild, Zielen und inneren Präferenzen (einschließlich sexueller)

- Neigung, sich auf intensive aber instabile Beziehungen einzulassen, oft mit der Folge von emotionalen Krisen

- Übertriebene Bemühungen, das Verlassenwerden zu vermeiden

- Wiederholt Drohungen oder Handlungen mit Selbstschädigung

- Anhaltende Gefühle von Leere.

Während die erste Kriteriengruppe auch für den „impulsiven Typus“ der emotional instabilen Persönlichkeit gilt (F60.30), müssen für den „Borderline-Typus“ (F60.31) zusätzlich Kriterien aus der zweiten Gruppe erfüllt sein (WHO 1993).

\subsubsection{DSM-IV-Kriterien für die BPS (APA 1994)}

Mindestens fünf von den neun folgenden Kriterien müssen erfüllt sein:

- Übermäßige, starke Wut oder Unfähigkeit, die Wut zu kontrollieren (z.B. häufige Wutausbrüche, ständige Wut oder häufige psychische Kämpfe)

- Chronische Leeregefühle

- Affektive Instabilität mit starken Stimmungsschwankungen

- Vorübergehende, stressbedingte Wahnvorstellungen oder schwere dissoziative Symptome

- Identitätsstörung: deutliches und dauerhaft bestehendes unbeständiges Selbstbild

- Häufig Suiziddrohungen, -andeutungen, -versuche oder Selbstverletzungen 
- Impulsivität bei mindestens zwei potentiell sich schädigenden Aktivitäten, ausgenommen suizidale oder selbstverletzende Handlungen (Drogen- und Alkoholabusus, Glücksspiel, Ladendiebstahl etc.)

- Unbeständige und intensive zwischenmenschliche Beziehungen, mit einem Wechsel zwischen den Extremen der Idealisierung und der Abwertung

- Verzweifeltes Bemühen, ein reales oder vorgestelltes Verlassenwerden zu vermeiden (APA 1994)

\subsection{Diagnostik der Borderline-Persönlichkeitsstörung}

Die Systematisierung und Operationalisierung der Störung geschah erstmals 1980 mit der Aufnahme in das DSM-III (APA 1980), das als ein multiaxiales Klassifikationssystem für psychiatrische Störungen dient. Das Schwergewicht bei der BorderlinePersönlichkeitsstörung liegt hierbei auf der Instabilität von Affekten und zwischenmenschlichen Beziehungen sowie auf Impulsdurchbrüchen (Dulz und Schneider 1996). Mit dem DSM-IV (APA 1994) wurden vorübergehende paranoide oder dissoziative Symptome als weiteres Diagnosekriterium aufgenommen.

Die „International Classification of Diseaeses“ der Weltgesundheitsorganisation (WHO 1993) übernahm weitgehend die phänomenologische und deskriptive Sichtweise des DSM-IV, ordnete die Borderline-Störung (F60.31) neben dem impulsiven Typus (F60.30) allerdings als Untergruppe der emotional instabilen Persönlichkeit (F60.3) zu (Bohus et al. 1999).

In dem heute gültigen DSM-IV (APA 1994) und in dem ICD-10-Klassifikationssystem (WHO 1993) erfolgt die Diagnosestellung bei Persönlichkeitsstörungen mittels expliziter Ein- und Ausschlusskriterien. Trotz vieler Gemeinsamkeiten sind die beiden Klassifikationssysteme ICD-10 und DSM-IV nicht direkt ineinander übersetzbar. So enthält die ICD-10, wie erwähnt, eine emotional instabile Persönlichkeitsstörung, die in einen impulsiven und einen Borderlinetypus unterteilt ist. Letzterer findet sich als eigenständige Borderline-Persönlichkeitsstörung im DSM-IV.

Bedenkt man, dass der impulsive Typus im DSM-IV diagnostisch auch der BorderlinePersönlichkeitsstörung entspräche, wird in der vorliegenden Arbeit im Einklang mit der internationalen Literatur statt von emotional instabiler Persönlichkeitsstörung nach 
ICD-10 bzw. Borderline-Persönlichkeitsstörung nach DSM-IV allgemein der Begriff Borderline-Persönlichkeitsstörung bzw. die generell gebräuchliche Abkürzung BPS Anwendung finden.

\subsection{Das klinische Erscheinungsbild der BPS}

Als zentrales Merkmal der BPS wird eine Störung der Affektregulation postuliert (Herpertz und Saß 2002). Patienten mit einer BPS reagieren bereits auf schwach ausgebildete emotionale Reize mit intensiven, aufschießenden Affektregungen. Sie neigen zu undifferenzierten dysphorischen Verstimmungen, die sie als quälend empfinden, da sie die Gefühle (z.B. Angst, Wut, Traurigkeit) voneinander nicht differenzieren können (Herpertz und Saß 2002). Oft kommt es zu inneren, aversiven Spannungszuständen, die als unerträglich erlebt werden (Stigmayr et al. 2001). Zwar kann es auch ohne Auslöser zu solchen Spannungszuständen kommen, doch sind meistens reale oder wahrgenommene Erfahrungen von Verlassen werden oder Zurückweisung typische Auslöser. Da zwischenmenschliche Nähe oft als bedrohlich wahrgenommen wird, können auch enge Beziehungen und Immunität solche Spannungen auslösen. Die aversiven Spannungszustände führen zu selbstschädigendem Verhalten, z.B Selbstverletzung, Essanfälle, Substanzmissbrauch, das eingesetzt wird, um die Spannungszustände zu lindern oder zu lösen. Das Erlebnis der negativen inneren Verstärkung führt zu einer baldigen Habituierung von selbstschädigendem Verhalten.

Die Abbildung 1 stellt den beschriebenen Kreislauf von Spannungsaufbau und Spannungslösung graphisch dar. 
Abbildung 1: Typischer Ablauf von Spannungsaufbau und Spannungslösung im Kontext von selbstschädigenden Verhaltensweisen bei der BPS (Abbildung nach Herpertz und Wenning, 2002)

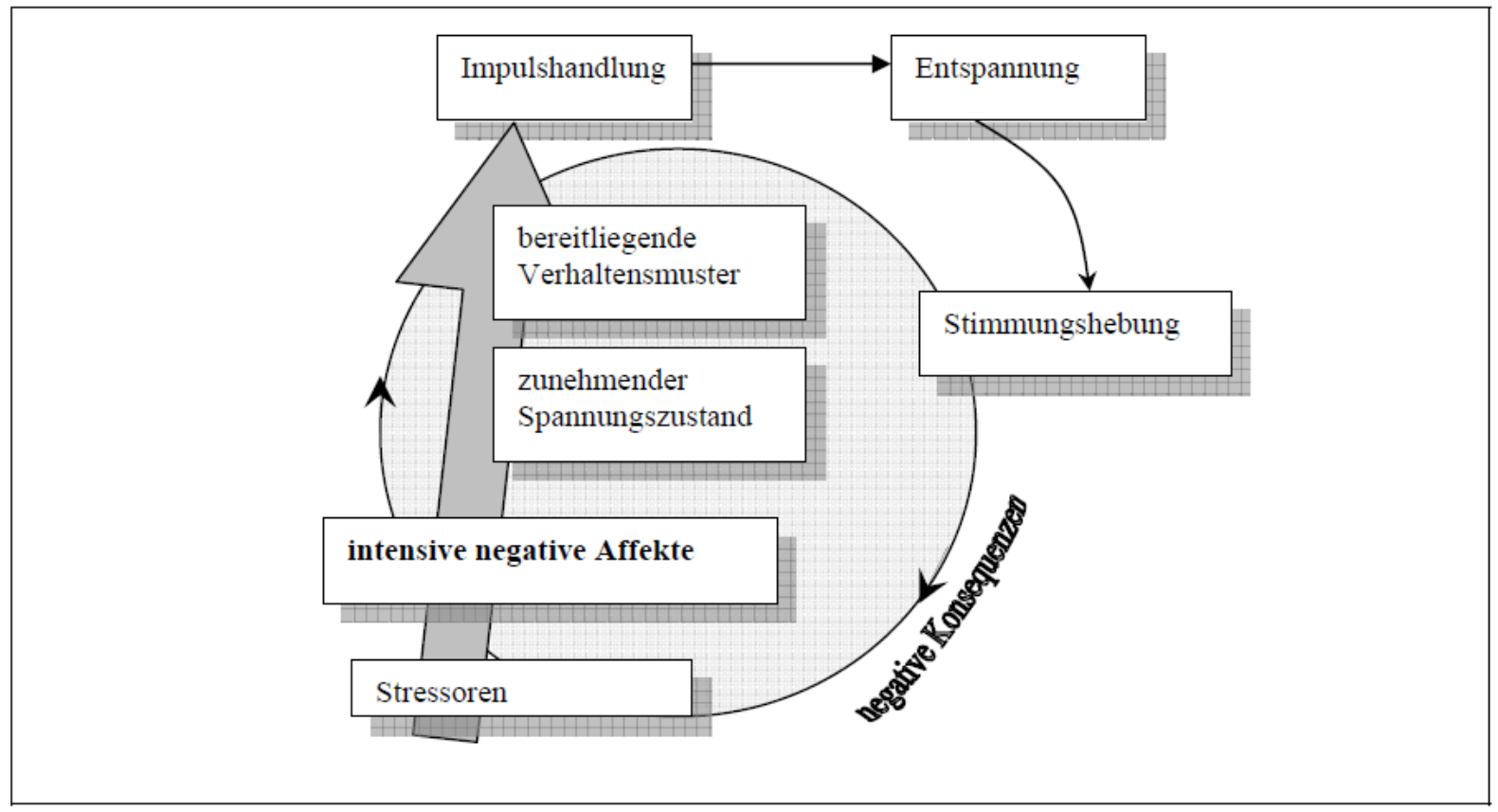

Im Gegensatz zu antisozialen Persönlichkeiten versuchen die Patienten mit einer BPS, Impulse zurückzuhalten oder zu unterdrücken (Herpertz und Saß 2002). Diese Versuche scheitern jedoch, da sie weder flexibel noch ausdifferenziert sind, noch liegt innen eine stabile Motivationslage zu Grunde. Daraus folgt ein Wechsel zwischen angespanntem Zurückhalten von Regungen und Impulsen und dem plötzlichen Ausleben von Affekt- und Handlungsimpulsen (Herpertz und Saß 1997)

Die Instabilität des Selbstbildes und der Selbstwahrnehmung drückt sich in einem Gefühl der Inkohärenz und in einer mangelnden Zukunftsorientierung und -planung aus. Die Folgen sind häufige Ausbildungsabbrüche und Stellenwechsel. Dem Selbstbild liegt meist ein negatives Selbstwertgefühl zu Grunde. Das Denkmuster der Patienten mit BPS entspricht häufig einem dichotomen „Schwarz-Weiß-Denken“. Bei besonderen Belastungen können Patienten mit einer Major Depression mit Schlafstörungen, Grübeln, Panik -und Angststörung reagieren (Herpertz und Saß 2002).

Ebenso können dissoziative Amnesien, Depersonalisationserlebnisse und pseudopsychotische Symptome beobachtet werden. Bei den pseudopsychotischen Symptomen handelt es sich gewöhnlich um angstintensive Zuspitzungen von Befürchtungen oder um intrusives Wiedererleben, die besonders in Zuständen 
ausgeprägter Dissoziation oder affektiver Erregung auftreten, bis hin zu passageren Wahneinfällen.

\subsection{Komorbiditäten}

Selten tritt die BPS isoliert auf. Die Prävalenz von Komorbidität ist bei der BPS groß. Häufige begleitende Störungen sind affektive Störungen, Essstörungen, Angststörungen, insbesondere die posttraumatische Belastungsstörung (PTBS), die Aufmerksamkeitsdefizit / Hyperaktivitätsstörung (ADHS) und Störungen in Zusammenhang mit Substanzmissbrauch. In einer Studie zur Prävalenz von Achse I-Störungen wurde bei 379 stationären Patienten mit einer BPS in 96,3\% der Fälle ein zusätzlicher Substanzmissbrauch diagnostiziert (Zanarini et al. 1998). Skodol et al. (1999) fanden bei 240 ambulanten Patienten mit einer BPS, dass 39,2\% die Kriterien einer zusätzlichen affektiven Störung erfüllten. Zimmerman und Mattia (1999) fanden bei 59 ambulanten Patienten mit einer BPS, dass mit einer Ausnahme alle eine komorbide DSM-IV Achse I-Störung aufwiesen und dass bei 69,5\% der Fälle mindestens vier komorbide Störungen aus der Achse I vorlagen.

Häufige Achse II-Überlappungen finden sich mit der histrionischen, der dependenten, der selbstunsicheren, der paranoiden und der schizotypischen PS.

Obwohl klare Unterschiede zwischen der BPS und der PTBS bestehen - z. B. dass der zeitliche Zusammenhang zwischen dem Auftreten der Symptomatik und dem auslösenden Ereignis nur bei der PTBS ein entscheidendes diagnostisches Kriterium darstellt und dass bei der PTBS normalerweise keine affektive Hyperreagibilität und kein selbstschädigendes Verhalten vorliegen - bleibt die Differentialdiagnose zur posttraumatischen Belastungsstörung schwierig (Bremner 1999). Dies trifft insbesondere dann zu, wenn eine frühe Traumatisierung vorlag, die zu einer andauernden Persönlichkeitsveränderung geführt haben könnte. In den letzten Jahren wird auch der Zusammenhang mit der ADHS immer häufiger diskutiert (Winkler et al. 2001; Dowson et al. 2004; Davids et al. 2005; Lampe et al. 2007). Deshalb wird in den folgenden Abschnitten auf die Komorbidität mit der PTBS und ADHS näher eingegangen. Aus der Literatur geht deutlich hervor, dass die BPS in den meisten Fällen mit einer komorbiden Achse I-Störung einhergeht. Ob die häufig zu beobachtende Komorbidität eher mit dem heterogenen Krankheitsbild der BPS zusammenhängt oder ob es sich tatsächlich um das gleichzeitige Auftreten von zwei 
ätiologisch differenzierbaren Störungen handelt (was der Definition von Komorbid entspricht), ist noch unklar (Paris 2005).

\subsubsection{BPS und PTBS}

Verschiedene Autoren schlagen vor, die BPS als eine Untergruppe der Angststörungen bzw. als komplexe oder chronifizierte PTBS zu betrachten. Bremner (1999) schlug vor, die BPS zusammen mit der PTBS und den dissoziativen Störungen in das Spektrum der traumatischen Störungen zusammenzufassen, da bei allen drei Störungsbildern die neurobiologischen Auswirkungen von Stress in der Pathogenese eine wichtige Rolle spielen. Nach Driessen et al. (2002) besteht eine deutliche Überschneidung in der Symptomatik einer BPS und einer PTBS entsprechend einer Typ-II-Traumatisierung, da BPS-Patienten häufig von multiplen und chronischen Traumatisierungen in Form von emotionaler Vernachlässigung, körperlicher Misshandlung und/oder sexuellem Missbrauch berichten. Traumaerfahrungen vom Typ-II scheinen für die Entstehung einer BPS eine sehr wahrscheinliche, aber keine notwendige Voraussetzung zu sein (Driessen et al. 2002). Allerdings wird die Typ-IITraumatisierung von etwa $80 \%$ der BPS-Patienten berichtet (Driessen et al. 2002). Driessen (2002) schlussfolgert daraus, dass die BPS eine Subgruppe der (komplexen) PTBS darstellen könnte. Diese Ansicht wird auch von anderen Autoren geteilt, hat sich aber noch nicht etabliert.

Bei der Typ II-Traumatisierung handelt es sich um mehrmalige, sich wiederholende oder andauernde Traumata. Sie unterscheidet sich dadurch von der Typ ITraumatisierung, die aus einem einzelnen, plötzlichen Ereignis von kurzer Dauer mit klarem Beginn und Ende besteht.

\subsubsection{Epidemiologie der BPS}

Die Lebenszeitprävalenz der BPS in der Allgemeinbevölkerung, d.h. die Häufigkeit in der Allgemeinbevölkerung, im Laufe eines Lebens an einer BPS zu erkranken, wird auf ca. $1,5 \%$ bis 4,6\% geschätzt (Bronisch 2001). Man geht davon aus, dass in Deutschland zur Zeit (2006) knapp eine Million Menschen an einer BPS leiden, 8\% bis $11 \%$ aller ambulanten psychiatrischen Patienten (Widiger und Weissmann 1991) und 
ca. $14 \%$ bis $20 \%$ der stationären psychiatrischen Patienten (Modestin et al. 1997) haben eine BPS Diagnose. In klinischen Populationen mit einer PS liegt die Prävalenz für eine BPS zwischen 30\% und 60\% (APA, DSM-IV, 1996). Unter Gefängnisinsassen oder Patienten in forensischen Kliniken ist die BPS nach der antisozialen PS die zweithäufigste Diagnose einer PS. Der Geschlechtsbias entsteht zum größten Teil durch die diagnostischen Kriterien. Da das selbstschädigende Verhalten für die Diagnose einer BPS und das streitsüchtige Verhalten für die Diagnose des impulsiven Typus vorliegen muss, wird die BPS eher bei Frauen und die impulsive PS eher bei Männern diagnostiziert.

\subsubsection{BPS und Suizidalität}

Die BPS ist die PS mit der höchsten Suizidrate. Die Angaben variieren von ca. 3\% (McGlashan 1986) bis ca. 10\% (Bohus und Remmel, 2004; Lieb et al. 2004), was eine 50-fach erhöhte Suizidrate gegenüber der Normalbevölkerung darstellt (Lieb et al. 2004). Nach Paris (2002) suizidiert sich einer von zehn Patienten mit einer BPS. Dabei findet sich die höchste Suizidrate bei BPS-Patienten im Alter zwischen 18 und 30 Jahren (Paris 2002). In einer Studie von Gunderson (1984) hatten aus einer Stichprobe von stationär behandelten Patienten mit einer BPS 75\% mindestens einen Suizidversuch im Laufe ihres Lebens begangen. Da bei anderen psychiatrischen Störungen Persönlichkeitseigenschaften wie Impulsivität und Aggression mit einem erhöhten Suizidrisiko korrelieren (Apter et al. 1993), scheint es wahrscheinlich, dass diese Merkmale auch bei der BPS zu einem erhöhten Suizidrisiko beitragen. Brodsky et al. (1997) untersuchten bei 214 Patienten mit einer BPS den Zusammenhang zwischen Impulsivität, sexuellem Missbrauch in der Kindheit und der Häufigkeit von Suizidversuchen und fanden, dass nicht die Ausprägung der BPS- Symptomatik, sondern die der Impulsivität mit dem suizidalen Verhalten korrelierte, auch dann, wenn weitere Faktoren (Lifetime Depression oder Substanzmissbrauch) kontrolliert wurden. Ein Zusammenhang zwischen Impulsivität und Missbrauchserfahrung in der Kindheit konnte in dieser Studie nicht gefunden werden, jedoch zeigte sich eine signifikante Korrelation zwischen dem Vorhandensein von Missbrauch in der Kindheit und der Häufigkeit von Suizidversuchen. Da mehrere Studien vorliegen, die eine Korrelation zwischen einem reduzierten Serotoninspiegel und einer erhöhten Impulsivität bei suizidalen Patienten (Roy et al. 1988; Linnoila et al. 1992), sowie zwischen 
aggressivem und suizidalem Verhalten bei anderen psychiatrischen Störungen suggerieren (Roy et al. 1988; Mann et al. 1997), schlagen die Autoren vor, vor allem die Impulsivität sowohl psychotherapeutisch, als auch medikamentös zu behandeln, um das suizidale Risiko zu reduzieren.

\subsection{Pathogenese der Borderline-Persönlichkeitsstörung}

Aufgrund der vielseitigen behaviouralen Auffälligkeiten und des Fehlens von klaren biologischen Markern, stellt die BPS für das Verständnis der psychobiologischen Grundlagen der Entstehung von Persönlichkeitstörungen eine besondere Herausforderungen dar (Clarkin et al. 2001).

\subsubsection{Genetik}

Prospektive Studien fanden ein zwischen 15,3\% und 23,4\% erhöhtes Risiko an einer BPS zu erkranken, wenn Familienangehörige ersten Grades die Diagnose einer BPS hatten (Silverman et al. 1991; Baron et al. 1985; Links et al. 1988). Togersen et al. $(1994,2000)$ konnten eine familiäre Häufung der BPS feststellen, die sich vor allem in der Vererbung von bestimmten Eigenschaften, nämlich der emotionalen Labilität, der Impulsivität und des selbstschädigenden Verhaltens zeigte. Torgersen et al. (2000) untersuchten 221 Zwillingspaaren und fanden bei 35\% der monozygoten und bei $7 \%$ der dizygoten Zwillingspaaren eine BPS.

\subsubsection{Psychosoziale Faktoren}

Da die Interaktion von Temperament und Umfeld eine zentrale Rolle in der Entwicklung von Selbstkontrolle (self-control), emotionaler Kontrolle (emotional control), Empathie und sozialem Verhalten spielt (Posner et al. 2005) und die Grundlage für die Ausbildung von Persönlichkeit darstellt, kommt dem sozialen Umfeld in der Erforschung der Pathogenese der BPS eine besondere Bedeutung zu. 
Mehrere Autoren vermuten, dass die interaktionellen Schwierigkeiten und die Störung der Selbstregulation bei der BPS aus einem gestörten Bindungsverhalten resultieren (Fonagy et al. 1996). Die Grundannahme der Bindungstheorie nach Bowlby (1969) geht davon aus, dass für die Entwicklung der emotionalen Gesundheit eines Kindes die Erfahrungen der interpersonellen Interaktionen entscheidend sind. Die moderne klinische Bindungsforschung bestätigt die Grundannahmen Bowlbys, da Hinweise vorliegen, die die Rolle dramatischer familiärer Beziehungen in der Ätiologie der BPS zeigen (Buchheim et al. 2002). Das relativ häufige Auftreten von Missbrauch, Trennung oder Vernachlässigung in der Kindheit bei Patienten mit einer BPS könnte im Zusammenhang mit der Entwicklung von unsicherem Bindungsverhalten bei diesen Patienten stehen (Posner et al. 2005). Signifikant gehäuft wurden Probleme im Bindungsverhalten zwischen Eltern und Kindern in BPS-Familien beobachtet (Paris et al. 1994). Patienten mit einer BPS wachsen häufig in einer chaotischen und feindseligen Familienatmosphäre auf und erfahren einen invalidierenden Erziehungsstil (Herpertz und Saß 2002), d.h., die Betroffenen wachsen in einem Umfeld auf, das ihre Gefühle, Gedanken und Verhalten nicht ernst nimmt und ständig in Frage stellt. Die Familieninteraktionen werden häufig als desorganisiert, chaotisch, konfliktreich und feindselig beschrieben (Links, 1992). Vor diesem Hintergrund, der dem Kind als Vorbild für seine Reifung und Entwicklung dient, können langfristige Zielorientierungen und moralische Normen nicht oder nur dysfunktional entwickelt werden (Herpertz und Saß 2002). Ein Zusammenhang zwischen Missbrauch, insbesondere sexuellem Missbrauch in der Kindheit und der Entwicklung einer BPS wurde in mehreren Studien bestätigt (Herman et al. 1989; Ogata et al. 1990; Zanarini, 1997). Herman et al. (1989) fanden, dass $81 \%$ der Patienten mit einer BPS Missbrauchs-erfahrungen in der Kindheit aufwiesen, sowohl körperliche (71\%) als auch sexuelle (67\%). Studien zu psychosozialen Risikofaktoren berichten signifikant häufiger von sexuellem sowie körperlichem Missbrauch, von schwerer körperlicher Vernachlässigung und von körperlicher Gewalt in der Kindheit von Patienten mit einer BPS im Vergleich zu Patienten mit anderen PSen (Paris et al. 1994). Die erste prospektive Studie mit einer Stichprobe von 639 Jugendlichen und ihren Müttern aus dem Staat New York konnte zeigen, dass Probanden mit nachgewiesenem körperlichen, sexuellen oder emotionalen Missbrauch oder schwerer Vernachlässigung mindestens viermal häufiger im jungen Erwachsenenalter unter einer PS leiden (Johnson et al. 1999). Unter Berücksichtigung der Komorbidität, 
fanden die Autoren nur für die BPS einen signifikanten Zusammenhang zwischen Missbrauch in der Kindheit und der PS (Zelkowitz et al. 2001). Sie fanden, dass Kinder, die einen sexuellen Missbrauch erlebt hatten, ein vierfaches Risiko zeigten, eine BPS zu entwickeln. Insbesondere über einen längeren Zeitraum sich erstreckende Erfahrungen von sexuellem Missbrauch und körperlichen sowie psychischen Misshandlungen führen bei Kindern und Jugendlichen, deren Entwicklung von Regulations- und Bewältigungsmechanismen noch nicht abgeschlossen ist, zu Störungen der adäquaten Affektsteuerung und der Verhaltenskontrolle. Resch (1996) gibt als typische Symptome nach Misshandlungen eine überschießende Reagibilität, expansive Verhaltensauffälligkeiten und eine verminderte Fähigkeit des verbalen Ausdrucks von emotionalem Erleben an. Durch das zusätzliche invalidierende, missachtende und kaum unterstützende oder berechenbare Umfeld wirken sich die traumatischen Erfahrungen stärker auf die Persönlichkeitsentwicklung aus (Herpertz und Saß 2002). Helgeland und Torgersen (2004) halten vor allem fünf psychosoziale Risikofaktoren für die Entstehung einer BPS verantwortlich: (1) Missbrauch, (2) Vernachlässigung, (3) ein instabiles Umfeld, (4) psychiatrisch erkrankte Eltern und (5) fehlende oder wenige Schutzfaktoren (z.B. höhere Schulbildung, Begabungen, durchschnittliche Intelligenz). Teicher et al. (2002) beschreiben in einem Übersichtsartikel bezüglich des Einflusses von Trauma und Stress in der Kindheit auf die neurobiologische Entwicklung, dass bei Personen mit Missbrauchserlebnissen eine verminderte Vernetzung der linken und rechten Hirnhemisphäre besteht. Die Autoren nehmen an, dass Probleme in der frühen Mutter-Kind- Interaktion die Integration der Funktionen der beiden Gehirnhälften beeinträchtigt, die das Kind daran hindert, die positiven und die negativen Eigenschaften der Bezugsperson in eine Repräsentation $\mathrm{zu}$ integrieren. Stattdessen verinnerlicht das Kind zwei widersprüchliche Bilder, wobei die positive Repräsentation in der linken Gehirnhälfte und die negative in der rechten Hemisphäre gespeichert werden. Diese gegensätzlichen Repräsentationen bleiben über die Entwicklung bis ins Erwachsenenalter bestehen und tragen dazu bei, dass eine Person in einem Zustand übermäßig positiv und einem anderen Zustand deutlich negativ wahrnimmt und beurteilt.

Zusammenfassend lässt sich sagen, dass auch nichtgenetische Faktoren eine große Rolle bei der Entwicklung der BPS spielen und vor allem aus ungünstigen Umgebungsfaktoren bestehen. Dabei sind Vernachlässigung und sexueller 
Missbrauch wichtige Faktoren. Wenn zusätzlich ein invalidierendes Umfeld vorliegt, ist es dem heranreifenden Kind kaum möglich, adäquate Regulations- und Bewältigungsmechanismen zu entwickeln, die für eine funktionale Affektsteuerung und Verhaltenskontrolle notwendig sind.

\subsection{Der Verlauf und die Prognose bei BPS}

Der Verlauf der BPS ist interindividuell sehr unterschiedlich. Es gibt jedoch Hinweise dafür, dass mit zunehmendem Alter die Symptome abklingen (McGlashan, 1986), was zu einer größeren Stabilität in persönlichen Beziehungen sowie beruflichen Situation beitragen könnte, die zwischen dem 30. oder 40. Lebensjahr beobachtet wurde (Bohus et al. 2000). In einer Langzeitstudie, in der 64 Patienten mit einer BPS über 27 Jahre lang betreut wurden, zeigte sich, dass $75 \%$ der Patienten im Alter von 35 - 40 Jahren zu einem normalen allgemeinen Funktionsniveau fanden. Im Alter von 50 Jahren waren es sogar $90 \%$ der Patienten, die ein normales allgemeines Funktionsniveau erreicht hatten (Paris und Zweig-Frank, 2001).

Eine hohe Intelligenz, Attraktivität, künstlerische Talente sowie begleitende anankastische Züge sind günstige prognostische Faktoren bei der BPS. Ungünstige Outcome-Kriterien sind eine Vorgeschichte mit elterlicher Gewalt und Inzest, begleitende schizotypische und antisoziale Persönlichkeitszüge, eine ausgeprägte Impulsivität und ein schlechtes prämorbides Funktionsniveau (Stone, 1993).

\subsection{Therapiemöglichkeiten bei der BPS}

Der Behandlungsbedarf von Patienten mit einer BPS ist ausgesprochen hoch. Lieb et al. (2004) geben einen Überblick über die Inanspruchnahme der Behandlungsmöglichkeiten von Seiten dieser Patientengruppe. Danach erhalten 97\% der BPSPatienten im Laufe ihres Lebens ambulante Hilfe von durchschnittlich sechs verschiedenen Psychotherapeuten. 95\% erhalten Einzeltherapie, 56\% Gruppentherapie und 42\% Familien- oder Paarberatung. 37\% erhalten Behandlung in einer Tagesklinik, $72 \%$ sind in stationärer psychiatrischer Behandlung. Die hohe Anzahl von Psychotherapeuten, auf die ein BPS-Patient im Durchschnitt im Laufe seines Lebens kommt, ist zum Teil durch die hohe Abbruchrate unter unspezifischen 
Behandlungsbedingungen zu erklären (ca. 75\%), bei der auch die emotionale Belastung der Therapeuten eine Rolle spielen dürfte (Bohus, 2002). Viele Studien zeigen einen geringeren Therapieerfolg sowie eine ungünstigere Prognose bei gleichzeitigem Vorliegen von mehreren Persönlichkeitsstörungen oder zusätzlichen Achse-I-Erkrankungen (Skodol et al. 2002).

\subsubsection{Psychotherapie}

Die Hauptziele einer Psychotherapie bei Patienten mit einer BPS sind eine Verbesserung der Affektregulation und Spannungstoleranz, die Verarbeitung traumatischer Erfahrungen und letztlich das Wiedererlangen einer selbstständigen Lebensführung und Arbeitsfähigkeit (Herpertz und Saß 2002). Zur Behandlung werden hauptsächlich die dynamische Psychotherapie, die kognitive Verhaltenstherapie, traumazentrierte sowie schulenübergreifende Ansätze eingesetzt.

Im Rahmen der psychodynamischen Ansätze wurde für die BPS und andere ClusterB- Persönlichkeitsstörungen die übertragungsfokussierte Psychotherapie (TFT, Transference- Focused Psychotherapy) (Clarkin et al. 2001) entwickelt, die annimmt, dass es sich bei den aktuellen Schwierigkeiten des Patienten um unbewusste Aktualisierungen pathologischer, internalisierter Beziehungen aus der Vergangenheit handelt. Das therapeutische Vorgehen besteht darin, eine tiefgreifende Veränderung der psychischen Struktur durch eine Lockerung der fixierten internalisierten Objektbeziehungen und eine Integration der abgespaltenen Selbst- und Objektrepräsentanzen herbeizuführen (Herpertz und Saß 2002). Im Rahmen der kognitiven Verhaltenstherapien hat sich besonders der Ansatz der DialektischBehaviouralen Therapie (DBT) nach Linehan $(1993,1996)$ als wirksam erwiesen, die bei der Behandlung von Patienten mit einer BPS unspezifischen Psychotherapien deutlich überlegen ist (Bohus und Berger, 1996).

Ausgehend von der biosozialen Theorie, dass die Ursache der BPS in der Interaktion zwischen einer konstitutionell bedingten gestörten Affektregulation und einer aus der Lernerfahrung resultierenden mangelhaften Emotionsregulation liegt, versucht die DBT spezielle Fertigkeiten zur Verbesserung der Spannungstoleranz, der Emotionsregulation und der sozialen Kompetenz und einer erhöhten Achtsamkeit zu vermitteln. Die neuen Strategien streben an, die maladaptiven Verhaltensmuster durch Einübung und Reflexion zu reduzieren. Auch die Schematherapie nach Young et al. 
(1988, 2005) versucht maladaptive Verhaltensmuster aktiv aufzubrechen. Die Schematherapie ist ein integratives Modell, das davon ausgeht, dass frühe, unerfüllte zentrale Bedürfnisse zu dysfunktionalen Schemata bezüglich der eigenen Person und der Beziehungen zu anderen Menschen führen. Schemata umfassen Erinnerungen, Emotionen, Kognitionen und Körperempfindungen und werden dann aktiviert, wenn Menschen mit Umgebungen konfrontiert werden, die sie an Situationen aus ihrer Kindheit erinnern, in denen das betreffende Schema entstanden ist. Es fand eine Erweiterung der theoretischen Konzepte und therapeutischen Strategien statt, da die Schematherapie ursprünglich nicht spezifisch zur Behandlung der BPS konzipiert wurde. Das Ziel war diese Behandlungsmethode dem Störungsbild der BPS besser anzupassen. Die Wirksamkeit einer psychotherapeutischen Behandlung der BPS ist mittlerweile durch mehrere Studien belegt (Binks et al. 2006; Giesen-Bloo et al. 2006; Linehan et al. 2006). Ihre Zahl ist jedoch begrenzt und der Nachweis beschränkt sich nur auf drei Psychotherapieformen (DBT; Mentalization Based Treatment nach Bateman et al. 1999, 2001 (MBT); Schema-fokussierte Therapie (SFT)). Die Wirksamkeit der DBT konnte durch fünf unabhängige Arbeitsgruppen in acht randomisierten kontrollierten Therapiestudien gezeigt werden. $\mathrm{Zu}$ den anderen Therapieformen liegen weniger randomisiert kontrollierte Studien vor (Bohus und Schmahl, 2006; Lieb et al. 2004). Trotz der positiven Ergebnisse zeigt sich, dass etwa die Hälfte der behandelten Patienten auf die angebotenen Verfahren ansprechen (Bohus und Schmahl, 2006). Welche Patienten auf welche Verfahren ansprechen, ist bisher unbekannt.

\subsubsection{Die Pharmakotherapie bei der BPS}

Zum aktuellen Zeitpunkt gibt es nur eine begrenzte Anzahl von randomisiert kontrollierten Studien zur BPS, die meist auf kleinen Stichproben und kurzen pharmakologischen Behandlungszeiträumen basieren (Bohus und Schmahl, 2006). Die Wirksamkeit folgender Medikamente wurde in den letzten zehn Jahren belegt: selektive Seoronin- Wiederaufnahmehemmer (SSRIs), Stimmungsstabilisatoren wie Valproinsäure sowie atypische Neuroleptika (Bohus und Schmahl, 2006). Ein zugelassenes Medikament zur Behandlung der BPS existiert derzeit nicht und die eingesetzten Medikamente dienen der mehr oder weniger effizienten Behandlung bestimmter Bereiche der Psychopathologie bzw. der komorbiden Störungen (Remmel und Bohus, 2006). Im Folgenden soll kurz erläutert werden, welche Medikamente zum 
aktuellen Zeitpunkt vorzugsweise zur Behandlung der verschiedenen Symptome (Depression, Angst, Fremdaggressivität, selbstverletzendes Verhalten, Suizidalität und dissoziative Symptomatik) zum Einsatz kommen.

Die Ergebnisse zur Behandlung der depressiven Symptomatik mit Fluoxetin sind widersprüchlich. Markovitz et al. (1991) fanden einen signifikanten Rückgang der Symptomatik bei der mit Fluoxetin behandelten BPS-Patienten im Vergleich zur Placebo- Gruppe. Die Studien von Salzman et al. (1995) und Coccaro und Kavoussi (1997) fanden keinen signifikanten Rückgang der Symptomatik. Bei der Behandlung der Angstsymptomatik bei Patienten mit BPS führte nur die Medikation durch Fluoxetin (Coccaro und Kavoussi, 1997; Markovitz et al. 1991) zu einem signifikanten Rückgang der Angstsymptome im Vergleich zu Haloperidol, Amitriptylin und Phenelzin, die dem Placebo nicht signifikant überlegen waren (Soloff et al. 1986, 1993). Die von Soloff et al. (1986, 1993) berichtete signifikante Behandlung der Impulsivität durch Haloperidol konnte nicht repliziert werden. Weder Amitriptylin noch Phenelzin waren der Behandlung durch Placebo überlegen. Zur Behandlung der Fremdaggressivität zeigte Fluoxetin die besten Ergebnisse (Coccaro und Kavoussi, 1997; Salzmann et al. 1995). Haloperidol war in der Behandlung der Fremdaggressivität Amitriptylin deutlich überlegen (Soloff et al. 1986). Die Überlegenheit von Haloperidol konnte im Vergleich zu Phenelzin jedoch nicht repliziert werden (Soloff et al. 1993). Für Topiramat bzw. Lamotrigin konnte keine signifikante Reduktion von Wut, Ärger und Aggressivität gefunden werden (Nickel et al. 2004, 2005; Tritt et al. 2005). Randomisierte, placebokontrollierte Pharmakastudien liegen bezüglich selbstverletzendes Verhalten nicht vor (Remmel und Bohus, 2006). In offenen klinischen Studien zeigten Fluoxetin (Markovitz et al, 1991) und Venlafaxin (Markovitz und Wagner, 1995) gute Ergebnisse, d.h. es konnte ein Rückgang der Selbstverletzungen beobachtet werden. Unter Carbamazepin, Trifluoperazin und Tranylcypromin wurden die Patienten mit BPS nach sechs Wochen im Fremdrating bezüglich Suizidalität signifikant besser eingeschätzt als die Patienten, die nur ein Placebo erhalten hatten (Cowdry und Gardner, 1988). In der Selbsteinschätzung jedoch führte keine der Substanzen zu einer Reduktion der Suizidalität. Die Behandlung mit Alprazolam musste vorzeitig beendet werden, da es zu einer Zunahme von schweren Kontrollverlusten und Suizidalität kam. Die dissoziative Symptomatik verringerte sich signifikant unter Naltrexon (Schmahl et al. 1999; Bohus et al. 1999). Unter Naloxon ergab sich keine signifikante Verbesserung der dissoziativen Symptomatik im Vergleich zu Placebo (Philipsen et al. 2004). 
Tabelle 2 Übersicht über bisherige Medikamentenstudien

Legende: K: kontrollierte Studie; O: offene Studie; F: Fallberichtsstudie

\begin{tabular}{|c|c|c|c|}
\hline Substanz & Autor / Jahr & Art & Haupteffekte auf \\
\hline Neuroleptika & & & \\
\hline \multirow[t]{3}{*}{ Haloperidol } & Soloff et al. 1986 & $\mathrm{~K}$ & \multirow{2}{*}{$\begin{array}{l}\text { Schizotype und affektive Symptome, Impulsivität, } \\
\text { klinisches Gesamtbild }\end{array}$} \\
\hline & Soloff et al. 1989 & $\mathrm{~K}$ & \\
\hline & Soloff et al. 1993 & K & Keine Wirkung \\
\hline Trifluperazin & $\begin{array}{c}\text { Cowdry und Gardner } \\
1988\end{array}$ & K & Depressivität, Angst, Suizidalität \\
\hline Thiothixen & Goldberg et al. 1986 & K & Psychotische, phobische und Zwangssymptome \\
\hline Flupenthixol D & $\begin{array}{l}\text { Montogomery und } \\
\text { Montogomery } 1982\end{array}$ & K & Suizidversuche reduziert \\
\hline \multirow[t]{3}{*}{ Clozapin } & $\begin{array}{l}\text { Frankenburg und } \\
\text { Zanarini } 1993\end{array}$ & $\mathrm{O}$ & \multirow{3}{*}{ Depressivität, selbst verletzendes Verhalten } \\
\hline & Benedetti et al. 1998 & $\mathrm{O}$ & \\
\hline & Chengappa 1999 & $\mathrm{O}$ & \\
\hline \multirow[t]{4}{*}{ Risperidon } & $\begin{array}{c}\text { Szigethy und Schulz } \\
1997\end{array}$ & $\mathrm{~F}$ & \multirow{4}{*}{ Selbstschädigung, klinisches Gesamtbild } \\
\hline & Rocca 2002 & $\mathrm{~F}$ & \\
\hline & Friedel 2008 & $\mathrm{O}$ & \\
\hline & Diaz-Marsa 2008 & $\mathrm{~F}$ & \\
\hline \multirow[t]{4}{*}{ Quetiapin } & Hilger et al. 2003 & $\mathrm{~F}$ & Impulsivität, klinisches Gesamtbild \\
\hline & Perella 2006 & $\mathrm{O}$ & Depressivität, Aggressivität \\
\hline & Villeneuve 2005 & $\mathrm{O}$ & Impulsivität \\
\hline & Van den Eynde 2007 & $\mathrm{O}$ & Impulsivität, Depressivität \\
\hline \multirow[t]{8}{*}{ Olanzapin } & Schulz et al. 1999 & $\mathrm{O}$ & Impulsivität, klinisches Gesamtbild \\
\hline & $\begin{array}{c}\text { Zanarini und } \\
\text { Frankenburg } 2001\end{array}$ & K & $\begin{array}{l}\text { Angst, Ärger, Feindseligkeit, Paranoia, } \\
\text { zwischenmenschliche Probleme }\end{array}$ \\
\hline & Zanarini et al. 2004 & $\mathrm{~K}$ & Chronische Dysphorie \\
\hline & $\begin{array}{l}\text { Bogenschutz und } \\
\text { Nurnberg } 2004\end{array}$ & K & klinisches Gesamtbild \\
\hline & Linehan 2008 & K & Reduktion von Aggressivität \\
\hline & Soler 2005 & K & Depression, Angst, Impulsivität \\
\hline & Pascual 2006 & $F$ & Agitiertheit (i.m) \\
\hline & Keshevan 2004 & $\mathrm{O}$ & psychotische Symptome, Depressionen \\
\hline \multirow[t]{2}{*}{ Ziprasidon } & Pascual 2008 & $\mathrm{~K}$ & kein Unterschied zu Placebo \\
\hline & Pascual 2006 & $\mathrm{~F}$ & Agitiertheit (i.m) \\
\hline Aripiprazol & Nickel 2006 & $\mathrm{~K}$ & Depression, Angst \\
\hline
\end{tabular}




\begin{tabular}{|c|c|c|c|}
\hline Substanz & Autor / Jahr & Art & Haupteffekte auf \\
\hline & Nickel 2007 & $\mathrm{~F}$ & Bestätigung der Ergebnisse der Vorstudie \\
\hline & Mobascher 2006 & $\mathrm{~F}$ & Gesamteindruck \\
\hline & Bellino 2008 & $\mathrm{~F}$ & Augmentation von Sertalin-Non-Respodern \\
\hline \multicolumn{4}{|l|}{ Antidepressiva } \\
\hline \multirow[t]{2}{*}{ Amitriptylin } & Soloff et al. 1986 & $\mathrm{~K}$ & \multirow{2}{*}{ Depressivität } \\
\hline & Soloff et al. 1989 & $\mathrm{~K}$ & \\
\hline \multirow[t]{2}{*}{ Imipramin } & Liebowitz et al. 1988 & $\mathrm{~K}$ & \multirow{2}{*}{ Atypische depressive Symptome } \\
\hline & Parsons et al. 1989 & K & \\
\hline Tranylcypromin & $\begin{array}{l}\text { Cowdry und Gardner } \\
1988\end{array}$ & K & Depressivität, Ärger, Impulsivität, Suizidalität \\
\hline Phenelzin & Soloff et al. 1993 & $\mathrm{~K}$ & Angst, Feindseligkeit \\
\hline \multirow[t]{3}{*}{ Fluoxetin } & Markowitz 1995 & $\bar{K}$ & klinisches Gesamtbild, Depressivität, Angst \\
\hline & Salzman et al. 1995 & K & Ärger, Aggressivität, Affektregulation \\
\hline & Cocarro et al. 1997 & K & Angst \\
\hline \multirow[t]{2}{*}{ Fluvoxamin } & Rinne et al. 2002 & K & Schnelle Stimmungsschwankungen \\
\hline & Van Vliet 1994 & $\bar{K}$ & ÄVPS schlechter beinflussbar \\
\hline \multirow[t]{3}{*}{ Sertralin } & Kavoussi et al. 1994 & $\mathrm{O}$ & Aggressivität, Irritierbarkeit \\
\hline & Markowitz 1995 & $\mathrm{~F}$ & $\begin{array}{l}\text { Depressivität, Dissoziation, Selbstschädigung, } \\
\text { Angst, Suizidalität }\end{array}$ \\
\hline & Katzelnick 1995 & $\mathrm{~K}$ & Soziale Phobie mit/ohne ÄVPS \\
\hline Venlafaxin & $\begin{array}{c}\text { Markowitz und Wagner } \\
1995\end{array}$ & $\mathrm{O}$ & $\begin{array}{l}\text { klinisches Gesamtbild, Stimmung, } \\
\text { Impulskontrolle }\end{array}$ \\
\hline \multicolumn{4}{|l|}{ Mood Stabilizer } \\
\hline \multirow[t]{3}{*}{ Carbamazepin } & $\begin{array}{l}\text { Gardner und Cowdry } \\
1986\end{array}$ & K & \multirow{2}{*}{$\begin{array}{l}\text { Suizidalität, Impulsivität, Ärger, klinisches } \\
\text { Gesamtbild }\end{array}$} \\
\hline & $\begin{array}{l}\text { Cowdry und Gardner } \\
1988\end{array}$ & K & \\
\hline & $\begin{array}{l}\text { De la Fuente und } \\
\text { Lotstra } 1994\end{array}$ & $\mathrm{O}$ & Depressivität, psychotische Symptome \\
\hline Oxcarbazepin & Bellino 2005 & $\mathrm{O}$ & Angst,Impulsivität,Gesamteindruck \\
\hline \multirow[t]{5}{*}{ Valproat } & Stein DJ et al. 1995 & $\mathrm{O}$ & $\begin{array}{l}\text { Affektregulation, Stimmung, klinisches } \\
\text { Gesamtbild }\end{array}$ \\
\hline & Wilcox 1995 & $\mathrm{O}$ & $\begin{array}{l}\text { Affektregulation, Fremdaggressivität, klinisches } \\
\text { Gesamtbild }\end{array}$ \\
\hline & $\begin{array}{l}\text { Frankenburg und } \\
\text { Zanarini } 2002\end{array}$ & K & $\begin{array}{c}\text { Zwischenmenschliche Sensibilität, Aggression, } \\
\text { Ärger/Feindseligkeit }\end{array}$ \\
\hline & Hollander et al. 2003 & K & klinisches Gesamtbild, Aggression, Depression \\
\hline & Hollander et al. 2005 & K & Impulsivität, Aggression \\
\hline Lamotrigin & Pinto und Akiskal 1998 & $\mathrm{~F}$ & Impulsivität, Suizidalität, klinisches Gesamtbild \\
\hline
\end{tabular}




\begin{tabular}{|c|c|c|c|}
\hline Substanz & Autor / Jahr & Art & Haupteffekte auf \\
\hline & Rizvi 2002 & $\mathrm{~F}$ & \\
\hline & Tritt 2005 & K & Ärgergefühle \\
\hline \multirow[t]{2}{*}{ Lithium } & Rifkin et al. 1972 & $\mathrm{~K}$ & \multirow{2}{*}{$\begin{array}{c}\text { Impulsivität, Stimmungsschwankungen, } \\
\text { Feindseligkeit }\end{array}$} \\
\hline & Sheard et al. 1976 & K & \\
\hline \multirow{2}{*}{ Topiramat } & Nickel 2005 & $\mathrm{~K}$ & Ärgergefühle \\
\hline & Nickel und Loew 2008 & $\mathrm{~F}$ & Ärgergefühle, Gewichtsabnahme \\
\hline Gabapentin & Paris 2007 & $\mathrm{O}$ & Gesamteindruck, Affekt, Ängstlichkeit \\
\hline \multicolumn{4}{|l|}{ Hypnotika/Sedativa } \\
\hline Clonazepam & Faltus 1984 & $\mathrm{~K}$ & klinisches Gesamtbild \\
\hline Lorazepam & Bick und Hannah 1986 & $\mathrm{~K}$ & Klinisches Gesamtbild \\
\hline Triazolam & Stein G 1992 & $\mathrm{~K}$ & klinisches Gesamtbild \\
\hline \multirow[t]{2}{*}{ Alprazolam } & $\begin{array}{c}\text { Gardner und Cowdry } \\
1985\end{array}$ & K & \multirow{2}{*}{ Erhöhung der Suizidalität und Aggressivität } \\
\hline & $\begin{array}{c}\text { Cowdry und Gardner } \\
1988\end{array}$ & K & \\
\hline \multicolumn{4}{|l|}{ Opiatantagonisten } \\
\hline \multirow[t]{4}{*}{ Naltrexon } & Sonne et al. 1996 & K & \multirow{4}{*}{ Autoaggression und Dissoziation } \\
\hline & McGee 1997 & $\mathrm{~K}$ & \\
\hline & Bohus et al. 1999 & $\mathrm{~K}$ & \\
\hline & $\begin{array}{c}\text { Griengl und } \\
\text { Dantendorfer } 2001\end{array}$ & $\mathrm{~F}$ & \\
\hline
\end{tabular}

Eine Übersichtsstudie von Zanarini (2004) fand (1), dass die meisten der in doppelblinden, placebokontrollierten Studien untersuchten Psychopharmaka effektiv waren und (2), dass die meisten dieser Medikamente in der Behandlung von Symptomen wie affektiver Dysregulation und impulsiver Aggression, die zentrale Symptome der BPS darstellen, nützlich waren. 
Tabelle 3 Medikamentenwirkungen bei der Borderline-Persönlichkeitsstörung Legende: $\mathrm{NL}=$ Neuroleptika; $\mathrm{MAO}-\mathrm{Hemmer}=$ Monoaminooxidasehemmer; SSRI = selektiver Serotonin-Wiederaufnahme-Hemmer; TZA = Trizyklisches Antidepressivum.

++ = übliche/deutliche Besserung; + = mögliche/leichte Besserung; +/- = kann verbessern oder verschlechtern; 0 = keine Wirkung; - = etwas verschlechtert; ? = noch keine Studien

* Diese Aussagen beziehen sich ausschließlich auf die kurz wirkenden Varianten.

\begin{tabular}{|c|c|c|c|c|}
\hline $\begin{array}{c}\text { Wirkung auf/ } \\
\text { Medikamentengruppe }\end{array}$ & Affekt & $\begin{array}{c}\text { Impulsivität/ } \\
\text { Selbstdestruktivität }\end{array}$ & $\begin{array}{c}\text { Kognition/ } \\
\text { Wahrnehmung }\end{array}$ & $\begin{array}{c}\text { Selbstbild/Rolle im } \\
\text { zwischenmenschlichen } \\
\text { Bereich }\end{array}$ \\
\hline Neuroleptika & + & + & + & 0 \\
\hline Atypische NL & + & + & + & $?$ \\
\hline MAO-Hemmer & ++ & $?$ & $?$ & + \\
\hline SSRI & ++ & ++ & 0 & 0 \\
\hline TZA & + & $+/-$ & $+/-$ & 0 \\
\hline Benzodiazepine & 0 & - & $?$ & 0 \\
\hline Mood Stabilizer & $+/-$ & ++ & + & $?$ \\
\hline Lithium & + & + & $?$ & $?$ \\
\hline Naltrexon & $?$ & + & $?$ & + \\
\hline
\end{tabular}

(modifiziert nach Gunderson 2005, S. 174)

\subsubsection{Symptomorientierte Therapiekonzepte}

Die pharmakologisch beeinflussbaren Symptome bei der BPS lassen sich in drei Domänen zusammenfassen: Impulsivität, Störungen der Affektivität und Störungen der Kognition. Soloff (1998) entwickelte Behandlungsalgorithmen für jede dieser Domänen, diese wurden von der American Psychiatric Association inzwischen in ihre offiziellen Behandlungsleitlinien für die Borderline-Persönlichkeitsstörung übernommen (APA 2001).

Für Patienten, die primär affektive Symptome wie Stimmungslabilität, Stimmungseinbrüche, unangebrachten Ärger, Angst, Zurückweisungssensitivität, innere Leere, Anhedonie, Dysphorie, Suizidalität und Depressivität zeigen, ist ein selektiver Serotonin-Wiederaufnahme-Hemmer oder ein verwandtes Antidepressivum wie Venlafaxin Mittel der ersten Wahl (siehe Abb. 2). Bei fehlender Response sollte ein zweiter SSRI oder ein anderes verwandtes Antidepressivum versucht werden. Sollte sich dann nach einer ausreichenden Behandlungsdauer immer noch kein oder nur ein partieller Effekt zeigen, kann, wenn Symptome wie Wut oder Ärger im 
Vordergrund stehen, ein niedrig dosiertes Neuroleptikum hinzugenommen werden oder aber ein langwirksames Benzodiazepin, wenn Angstgefühle dominieren. Bringt dieses Vorgehen keinen ausreichenden Erfolg kann man zu Monoaminooxidasehemmern wechseln und bei fehlender Response einen Einsatz von Carbamazepin, Valproat oder Lithium erwägen.

Zeigt der Patient vorrangig impulsive Symptome (siehe Abb. 2) wie Suizidandrohungen, Selbstverletzungen, Aggressivität, Feindseligkeit, geringe Frustrationstoleranz und Suchtverhalten, ist ebenfalls ein SSRI Mittel der ersten Wahl. Bei unbefriedigendem Effekt sollte auf ein niedrig dosiertes Neuroleptikum gewechselt werden bzw. ein solches hinzugefügt werden. Ein Wechsel zu Monoaminooxidasehemmern oder ein zusätzlicher Einsatz von Lithium sollte bei fortbestehender Symptomatik bedacht werden. Mood Stabilizer wie Carbamazepin und Valproat stellen bei fehlender Response eine weitere Option dar. Ist auch hierdurch keine wesentliche Besserung zu erreichen, kann als letzte Option zusätzlich ein atypisches Neuroleptikum hinzugenommen werden.

Zeigt der Patient primär kognitiv-perzeptionelle Symptome (siehe Abb. 2) wie Misstrauen, paranoide Ideen, Halluzinationen, Derealisation oder Depersonalisation, sollte mit einem niedrig dosierten Neuroleptikum begonnen werden. Bei ausbleibendem Erfolg kann zunächst die Dosis gesteigert werden. Zeigt sich mit der Dosissteigerung keine ausreichende Besserung, kann bei bestehenden affektiven Symptomen auf ein SSRI oder in zweiter Linie auf einen Monoaminooxidasehemmer gewechselt werden. Findet man hingegen bei dem Patienten wenig affektive Symptome, sollte man auf ein atypisches Neuroleptikum wechseln.

Bei der Therapieplanung sollte man grundsätzlich zuerst eine Monotherapie anstreben, bevor bei unzureichendem Erfolg eine Kombinationstherapie überlegt werden kann. Die Gabe von Benzodiazepinen sollte immer nur vorübergehend sein und kritisch überlegt werden (APA 2001; Soloff 2000; Wedekind et al. 2005). 


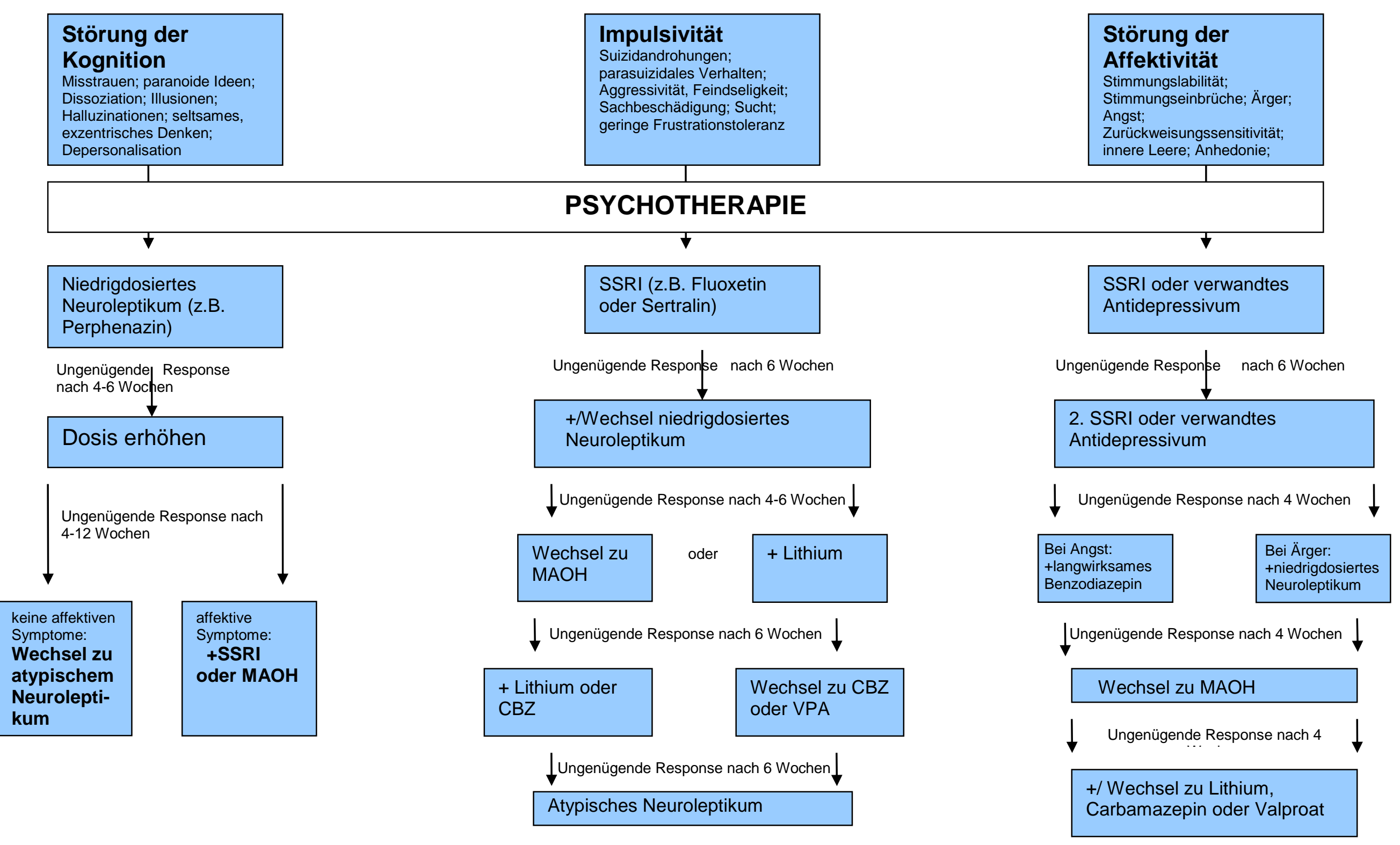

Abbildung 2 Behandlungsalgorithmus bei BPS bei verschiedenen Syndromkomplexen (modifiziert nach APA 2001; Soloff 1998; Wedekind et al. 2005)

Legende: $\quad \mathrm{CBZ}=$ Carbamazepin, $\mathrm{VPA}=$ Valproat, $\mathrm{SSRI}=$ selektiver Serotonin-Wiederaufahme-Hemmer, MAO-Hemmer $=$ Monoaminooxidasehemmer 


\subsubsection{Hypothesengenerierung und Zielsetzung}

Im klinischen Alltag lässt sich die Beobachtung machen, dass BPS-Patienten besonders häufig, anhaltend und intensiv die Ressourcen von psychiatrischen Institutionen beanspruchen. So stellen Borderline-Patienten einen wesentlichen Anteil an der klinisch psychiatrischen Population, was sich auch in der Tatsache zeigt, dass die BPS die häufigste Persönlichkeitsstörung bei Patienten in psychiatrischen Kliniken ist.

Die BPS gilt für viele Psychiater und Psychotherapeuten als allgemein schwer behandelbar oder sogar behandlungsrefraktär. Therapeutische Interventionsmöglichkeiten werden immer wieder heftig diskutiert: Psychotherapie, Psychopharmakotherapie oder Beides?

Nach herrschender Lehrmeinung ist die Psychotherapie heutzutage die Basis und der Standard der Behandlung der BPS. Etliche Studien widmeten sich der Psychotherapieforschung, allerdings konnte bislang hauptsächlich die von Linehan entwickelte DBT ihre Wirksamkeit in randomisierten kontrollierten Studien unter Beweis stellen.

BPS-Patienten werden im klinischen Alltag aber erfahrungsgemäß auch intensiv medikamentös versorgt. Aufgrund der schwerwiegenden und vielseitigen klinischen Symptomatik und einer hohen Morbidität und Mortalität gerät die BPS auch zwangsläufig immer wieder in den Fokus der Psychopharmakotherapie; so ist die BPS mit einer Suizidrate von 10\% eine der psychiatrischen Erkrankungen mit der höchsten Sterblichkeit.

Betrachtet man die empirische Datenlage bezüglich Medikamentenstudien, gibt es zwar in zunehmenden Maß kontrollierten Studien, die Effekte auf einzelne Symptome zeigen konnten im Vergleich zu anderen psychiatrischen Störungen, allerdings ist die Anzahl (offene und kontrollierte Studien) relativ gering und es fehlen weitgehend Evidenz-basierte und etablierte pharmakologische Leitlinien. Folglich herrscht im klinischen Alltag eine große Unsicherheit im Hinblick auf eine sinnvolle Psychopharmakotherapie, häufig basiert die Verschreibungspraxis mehr auf individueller praktischer Erfahrung als auf Evidenz-basierter Medizin.

Bezüglich der Psychopharmakotherapie bei der BPS ergeben sich somit drei große Fragestellungen: 
(1) Wie häufig bekommen Borderline-Patienten eine Psychopharmakotherapie in der universitären psychiatrischen Versorgung?

(2) Welche Substanzklassen und Wirkstoffe werden in welchen Dosierungen gegeben und entsprechen diese den Literaturempfehlungen?

(3) Gab es in den letzten beiden Jahrzehnten Veränderungen in der Verschreibungspraxis von Psychopharmaka bei der BPS und ist eine rationale Evidenz basiertes Procedere zu erkennen?

Um diesen Fragen nachzugehen, sollten im Rahmen der vorliegenden Arbeit Medikamentenanamnesen und Behandlungsverläufe von 2008 - 2012 aus Patientenakten der Klinik für Psychiatrie und Psychotherapie retrospektiv erfasst werden und mit den Behandlungsverläufe von 1996- 2004 verglichen werden.

Weiterhin interessant erscheint, ob sich die Häufigkeit einer Medikamentengabe oder die Art der verordneten Medikamente bei verschiedenen Behandlungsmodalitäten oder verschiedenen Syndromkomplexen signifikant in diesem Zeitraum unterscheidet. Folglich sollte geprüft werden, wie häufig Patienten welche Modi an stationären Therapien in Anspruch nehmen, mit welchen Syndromkomplexen und weshalb sie in die Behandlung kommen und welche Auswirkungen dies auf die erfolgte Psychopharmakotherapie hat.

Im Kontext sollten zusätzlich zu diesen Untersuchungen wichtige soziodemographische Parameter und Komorbiditäten erfasst werden, um eine genauere Beschreibung der Stichprobe zu ermöglichen.

Auch hier soll geprüft werden, ob sich dies bezügliche Charakteristika der Patientengruppen zwischen den beiden Untersuchungszeiträumen unterscheiden. 


\section{Methoden}

Nach der Genehmigung der Studie durch die lokale Ethikkomission wurde bei 87 stationär behandelten Patienten mit BPS die durchgeführte Psychopharmakotherapie retrospektiv erfasst. Genaues Augenmerk wurde auf Unterschiede in der Psychopharmakotherapie verschiedener Behandlungsmodi und vorliegender Syndromkomplexe gelegt. Ferner wurden die Soziodemographie, psychiatrische Komorbiditäten, die Dokumentation der diagnostischen Kriterien und verschiedene Aufenthaltsparameter, wie der Aufnahmeanlass, erhoben. Mit Hilfe eines von der Arbeitsgruppe entwickelten Fragebogens wurden Daten aus den Krankenakten der Patienten ermittelt, die im Zeitraum von 2008 bis 2012 wegen der Hauptdiagnose einer Borderline- Persönlichkeitsstörung (F60.3 nach ICD-10) in der stationären Behandlung in der Klinik für Psychiatrie und Psychotherapie der Universität Göttingen waren.

Meine Aufgabe bei der vorliegenden Studie war es die Auswertung der erhobenen Daten vorzunehmen und diese Daten statistisch auszuwerten und zu interpretieren.

\subsection{Die Patientenrekrutierung}

Mit Hilfe des Rechenzentrums der Universität Göttingen wurden die Patienten ermittelt, die unter der Diagnose einer emotional instabilen Persönlichkeitsstörung (F60.3 nach ICD-10) im Zeitraum von 2008 bis 2012 an der Klinik für Psychiatrie und Psychotherapie an der Universität Göttingen stationär behandelt und abgerechnet wurden $(n=87)$.

\subsection{Das Erhebungsinstrument}

Zur Datenerhebung wurde von der Arbeitsgruppe zunächst ein mehrfach überarbeiteter Fragebogen entwickelt (siehe Anlage). Im Fragebogen wurden alle psychiatrisch- oder psychotherapeutisch-stationären Aufenthalte der Patienten im Zeitraum von 2008-2012 mit Dauer und Behandlungsmodalitäten dokumentiert, sei es an der Universität Göttingen oder an anderen Kliniken. Zu den einzelnen Aufenthalten 
wurden verschiedene Parameter erhoben. So zum Beispiel der Aufnahmeanlass, der Syndromkomplex bei Aufnahme, Suizidversuche oder Selbstverletzungen vor- oder während der stationären Behandlung, gesetzliche Betreuung vor und nach dem Aufenthalt, oder der Entlassungsmodus soweit dies erfassbar war.

Hinsichtlich der oben genannten Parameter wurde nachfolgend ein Vergleich der Zeitintervalle, von 1996 bis 2004 und von 2008 bis 2012 durchgeführt, welche dazu diente, Unterschiede in der praktizierten Therapie sichtbar zu machen. Überdies wurden weitere Behandlungsvariablen, wie nicht strukturierte Therapien (z.B. Physiotherapie) und eventuell erfolgte strukturierte Psychotherapien, dokumentiert. Der Fragebogen umfasst weiterhin soziodemographische Gesichtspunkte wie:

- Partnerschaft und Ehe

- Wohnsituation der Patienten

- ausgeübte Tätigkeit, Ausbildung und Abschluss der Patienten

- psychiatrische Erkrankungen in der Familie.

Ferner wurde untersucht, ob die einzelnen diagnostischen Kriterien nach ICD-10 (WHO 1993) und DSM-IV (APA 1994) bei Patienten dokumentiert waren und ob weitere Diagnosen einschließlich Substanzmissbrauch festgestellt wurden. Die Zahl der Suizidversuche in der Krankengeschichte wurde festgehalten.

Bearbeitet wurden die Fragebögen anhand von Daten, die durch Einsicht in die Akten der betroffenen Patienten gewonnen werden konnten. Relevant waren dabei die Daten aus Arztbriefen und -aufzeichnungen, stationären Behandlungsprotokollen, Patientenaufzeichnungen und anderweitigen Dokumentationen.

\section{$\underline{\text { Statistik }}$}

Patientencharakteristika und soziodemographische Daten wurden zunächst deskriptiv dargestellt, ebenso wie die Häufigkeit und die Dosierungen der verordneten Pharmaka. Es wurden anschließend Mittelwertvergleiche der Häufigkeit einzelner verordneter Pharmaka und deren durchschnittliche Dosierungen, im Hinblick auf Änderungen über den individuellen stationären Behandlungsverlauf, durchgeführt. Zum Vergleich der Daten aus dem hier untersuchten Zeitraum 2008-2012 lagen entsprechende Vergleichswerte einer früheren Studie der Arbeitsgruppe vor, die unter ähnlicher Fragestellung aus dem Zeitraum 1996-2004 untersucht hatte. 
Datenvergleiche zwischen den nominal skalierten Werten wurden mit Hilfe des $X^{2}-$ Tests und bei intervallskalierten Daten wurde der t-Test angewandt. Häufigkeitstabellen wurden mit Hilfe von STATISTICA aufgestellt. 


\section{Ergebnisse}

\subsection{Patientencharakteristika}

Das Kriterium für die Aufnahme einer Person in die Stichprobe war die (Vergabe der) Diagnose „Emotional instabile Persönlichkeitsstörung, impulsiver oder Borderline-Typ“ gemäß ICD-10 (F60.30 und F 60.31) während einer stationären Behandlung in der Klinik für Psychiatrie und Psychotherapie der UMG im Zeitraum 2008-2012.

Beide Geschlechter wurden für die Untersuchung retrospektiv ausgewertet, 86,2 \% Frauen und 13,8\% Männern befanden sich in der Studienpopulation, die insgesamt 87 Patienten umfasste. Das Durchschnittsalter bei der Erstaufnahme lag bei 30,5 Jahren (SD 10,7; Spanne 19-62).

Die Patienten wurden innerhalb des Untersuchungsintervalls durchschnittlich über einen Zeitraum von 1,4 Jahren (SD 0,7 Jahre) beobachtet, das heißt von der ersten dokumentierten stationären Aufnahme bis zur Entlassung aus dem letzten dokumentierten Aufenthalt, an der Klinik für Psychiatrie und Psychotherapie der Universität Göttingen. Patienten befanden sich durchschnittlich 22,7 Tage (SD 34,6) in stationärer Behandlung in diesen Kliniken.

\subsubsection{Soziodemographie}

Im Folgenden werden die erhobenen demographische Daten beschrieben (siehe Tabelle 1): 83\% der Personen waren ledig, 27,6\% lebten in einer Partnerschaft, 9,2\% waren verheiratet, weitere $6,9 \%$ waren geschieden. Fast alle Personen beschrieben sich als heterosexuell.

Die Mehrheit der Patienten war kinderlos. Waren Kinder vorhanden, lebten diese allerdings häufig nicht bei den Patienten. Viele lebten stattdessen beim getrennt lebenden Partner oder kamen in Pflegefamilien bzw. bei Verwandten unter; einige lebten schon alleine.

Die Patienten selbst wohnten zur knappen Hälfte alleine, 21,8\% wohnten zusammen mit dem Partner.

Im Heim oder betreutem Wohnen lebten 9,2\% der Patienten. 
Als höchsten erzielten Schulabschluss hatten 18,5\% Patienten das Abitur. 30,9\% hatten die Realschule, 39,5\% Patienten die Hauptschule abgeschlossen. 7,4\% der Patienten besaßen keinen Abschluss.

\section{Tabelle 4 Soziodemographie}

\begin{tabular}{|c|c|c|}
\hline & Prozent & Häufigkeit \\
\hline Ehe & \multicolumn{2}{|c|}{$\mathrm{n}=87$} \\
\hline Nein & $83,9 \%$ & 73 \\
\hline $\mathrm{Ja}$ & $9,2 \%$ & 8 \\
\hline Geschieden & $6,9 \%$ & 6 \\
\hline Partnerschaft & \multicolumn{2}{|c|}{$n=87$} \\
\hline $\mathrm{Ja}$ & $27,6 \%$ & 24 \\
\hline Nein & $72,4 \%$ & 63 \\
\hline Häufiger Partnerwechsel & \multicolumn{2}{|c|}{$\mathrm{n}=70$} \\
\hline Nein & $67,1 \%$ & 47 \\
\hline $\mathrm{Ja}$ & $32,9 \%$ & 23 \\
\hline Sexuelle Ausrichtung & \multicolumn{2}{|c|}{$n=87$} \\
\hline Heterosexuell & $89,7 \%$ & 78 \\
\hline Homosexuell & $10,3 \%$ & 9 \\
\hline Bisexuell & $0,0 \%$ & 0 \\
\hline Wohnsituation & \multicolumn{2}{|c|}{$\frac{1}{n=87}$} \\
\hline Allein & $43,7 \%$ & 38 \\
\hline Mit Partner & $21,8 \%$ & 19 \\
\hline Bei Eltern & $17,2 \%$ & 15 \\
\hline Betreutes Wohnen & $9,2 \%$ & 8 \\
\hline Stark wechselnd & $1,1 \%$ & 1 \\
\hline Im Heim & $1,1 \%$ & 1 \\
\hline Kein fester Wohnsitz & $1,1 \%$ & 1 \\
\hline Anderes & $4,6 \%$ & 4 \\
\hline Tätigkeit & \multicolumn{2}{|c|}{$\mathrm{n}=85$} \\
\hline Arbeitslos & $32,9 \%$ & 28 \\
\hline Student & $10,6 \%$ & 9 \\
\hline In ungelernter Arbeit & $8,2 \%$ & 7 \\
\hline Im gelernten Beruf & $8,2 \%$ & 7 \\
\hline Auszubildender & $14,1 \%$ & 12 \\
\hline In Rente & $7,1 \%$ & 6 \\
\hline Hausfrau & $1,2 \%$ & 1 \\
\hline Schüler & $8,2 \%$ & 7 \\
\hline Erwerbsunfähig & $3,5 \%$ & 3 \\
\hline Arbeitsunfähig & $3,5 \%$ & 3 \\
\hline Häufig wechselnd & $1,2 \%$ & 1 \\
\hline In Umschulung & $1,2 \%$ & 1 \\
\hline
\end{tabular}




\begin{tabular}{|l|c|c|}
\hline Schulischer Abschluss & $39,5 \%$ & 32 \\
\hline Hauptschule & $30,9 \%$ & 25 \\
\hline Realschule & $18,5 \%$ & 15 \\
\hline Abitur & $7,4 \%$ & 6 \\
\hline Keine & $2,5 \%$ & 2 \\
\hline Noch in Ausbildung & $1,2 \%$ & 1 \\
\hline Sonderschule & Prozent & Häufigkeit \\
\hline \multicolumn{3}{|c|}{$\mathbf{n = 8 1}$} \\
\hline Beruflicher Abschluss & $45,7 \%$ & 37 \\
\hline Keiner & $25,9 \%$ & 21 \\
\hline Noch in Ausbildung & $24,7 \%$ & 20 \\
\hline Abgeschlossene Ausbildung & $3,7 \%$ & 3 \\
\hline Abgeschlossenes Studium & $\mathbf{n}=85$ \\
\hline $\begin{array}{l}\text { Verwandte mit psychiatrischen } \\
\text { Diagnosen }\end{array}$ & $38,8 \%$ & 33 \\
\hline Ja & $61,2 \%$ & 52 \\
\hline Nein & \multicolumn{2}{|c|}{$\mathbf{n = 8 5}$} \\
\hline Verwandte mit Hinweisen auf eine BPS & $42,4 \%$ & 36 \\
\hline Ja & $57,6 \%$ & 49 \\
\hline Nein
\end{tabular}

Die oben aufgeführte Tabelle zeigt die bisherige berufliche Qualifikation der Untersuchten. 32,9\% der Patienten waren nie erwerbstätig oder befanden sich zum Zeitpunkt der Studie in einer Ausbildung bzw. Lehre. 32\% waren arbeitslos oder ungelernt arbeitstätig.

Familienanamnestisch ließen sich bei mehr als einem Drittel der Patienten psychiatrische Erkrankungen bei Verwandten ersten Grades eruieren. Der Großteil der Patienten (42,4\%) beschrieb auch Symptome bei Verwandten ersten Grades die auf eine BPS hinwiesen.

\subsubsection{Psychiatrische Komorbiditäten}

Neben der Emotional- instabilen -Persönlichkeitsstörung, erfüllten 6\% der Patienten auch Kriterien wenigstens einer weiteren Persönlichkeitsstörung. Bei $40 \%$ der Patienten standen psychische Störungen und Verhaltensstörungen durch psychotrope Substanzen (ICD-10 F 1X.x) im Vordergrund. Zu erwähnen ist allerdings, dass bei der Dokumentation dieser Störungen auch Patientenangaben, die zum Beispiel während Arztgesprächen geäußert und bekannt wurden, mit ausgewertet wurden und nicht nur ärztlich gestellte Diagnosen in Arztbriefen wie bei anderen psychischen Störungen. Die Diagnose einer Somatoformen Störung wurde bei 40 \% der Patienten gestellt. 31\% 
litten unter einer affektiven Störung, im Wesentlichen waren dies einzelne oder rezidivierende depressive Episoden. Belastungs- und Anpassungsstörungen (F43) wurden bei $28 \%$ der Untersuchten diagnostiziert, es kann nicht gesagt werden, welchen Anteil hierbei Posttraumatische Belastungsstörungen (ICD-10 F 43.1) hatten. 14\% der Patienten litten an Essstörungen, wobei auch anamnestische Angaben mit berücksichtigt wurden. Andere Verhaltens- und emotionale Störungen mit Beginn in der Kindheit und Jugend, Entwicklungsstörungen und Intelligenzminderung wurden bei $10 \%$ der Patienten erhoben. 
Abbildung 3 Komorbiditäten (ärztlich gestellte Diagnosen; die Angaben zu Substanzmissbrauch und Essstörungen beruhen zusätzlich auf Patientenangaben) $\mathrm{N}=86$ Patienten Legende: dunkle Balken: Störungshauptgruppen nach ICD-10; helle Balken: Störungsuntergruppen nach ICD-10

Psychische und Verhaltensstörungen d. psychotrope Substanzen, F1x (Patientenangaben, $\mathrm{n}=34$ )

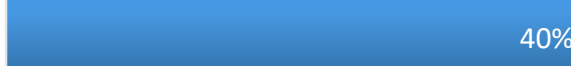

Neurotische, Belastungs- und somatoforme Störungen, $\mathrm{F} 4 \mathrm{x}(\mathrm{n}=34)$

Reaktionen auf schwere Belastungen und Anpassungsstörungen, F43 ( $n=24)$<smiles>C1CCCCC1</smiles>

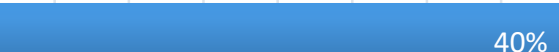

Phobische, Angst- und Zwangsstörungen, F40-42 ( $n=18$ )

weitere Persönlichkeits-und Verhaltensstörungen, F6x $(n=5)$

Dissoziative Störungen, F44 ( $n=3)$

Somatoforme Störungen, F45 ( $n=2)$

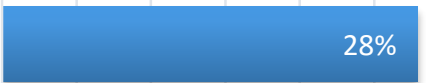

Affektive Störungen, F3x ( $n=27)$

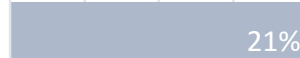

Essstörungen, F50 (Patientenangaben, $\mathrm{n}=12$ )

Verhaltungsauffälligkeiten in Verbindung mit körperlichen Störungen und Faktoren, F5x ( $n=12)$

Verhaltens- und emotionale Störungen mit Beginn in der Kindheit und Jugend, F9x $(n=9)$

Entwicklungsstörungen, $\mathrm{F} 8 \mathrm{x}(\mathrm{n}=3)$
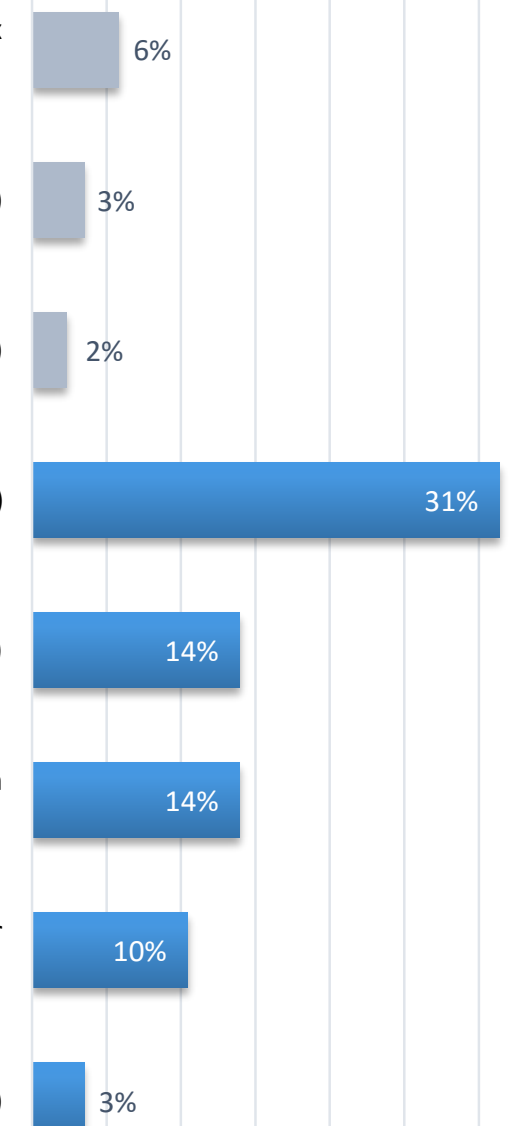

$$
\text { ) }
$$




\section{Abbildung 4 Komorbiditäten - Psychische und Verhaltensstörungen durch psychotrope Substanzen ( $\mathrm{N}=87$ Patienten)}

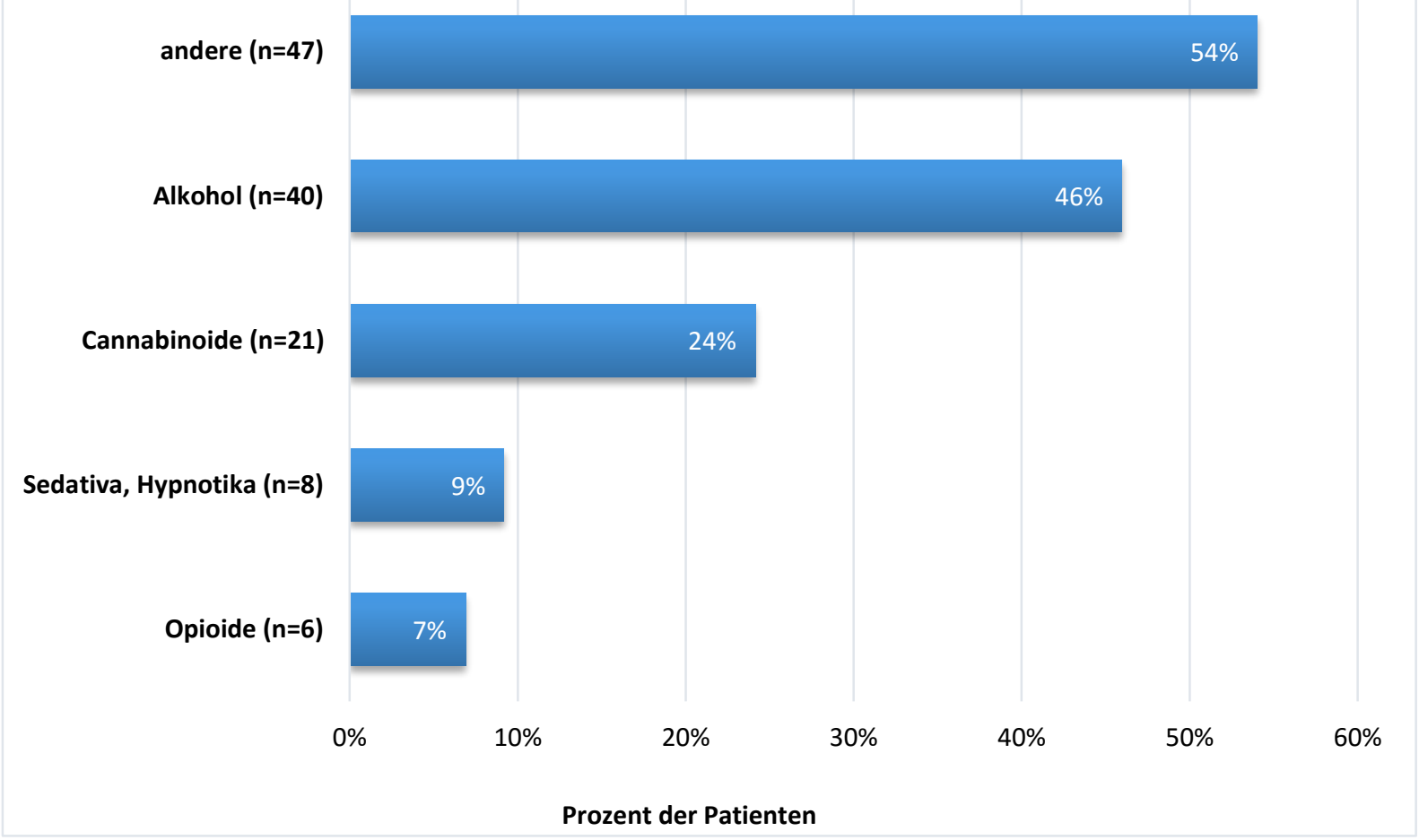

Aus dem Bereich der Substanz bezogenen Störungen (vorrangig schädlicher Gebrauch und Abhängigkeiten) fand man bei $46 \%$ der Patienten Störungen durch Alkohol, bei 24 \% Störungen durch Konsum von Cannabinoiden, während Störungen durch Sedativa und Hypnotika wiesen $9 \%$ der Patienten auf.

Bei psychischen und Verhaltensstörungen durch psychotrope Substanzen kann unterschieden werden zwischen schädlichen Gebrauch oder Abusus, und einem Abhängigkeitssyndrom. Betrachtet man die einzelnen Substanzen finden sich hierbei unterschiedliche Verhältnisverteilungen.

Tabelle 5 Verhältnis von Abhängigkeit und Abusus bei verschiedenen Substanzen

\begin{tabular}{|l|c|c|c|c|}
\hline & $\begin{array}{c}\text { Opioide } \\
(\mathrm{n}=6)\end{array}$ & $\begin{array}{c}\text { Sedativa oder } \\
\text { Hypnotika }(\mathrm{n}=8)\end{array}$ & $\begin{array}{c}\text { Alkohol } \\
(\mathrm{n}=40)\end{array}$ & $\begin{array}{c}\text { Cannabinoide } \\
(\mathrm{n}=21)\end{array}$ \\
\hline Abusus & $67 \%$ & $62 \%$ & $67 \%$ & $81 \%$ \\
\hline Abhängigkeit & $33 \%$ & $38 \%$ & $33 \%$ & $19 \%$ \\
\hline
\end{tabular}




\subsubsection{Suizidversuche}

$56 \%$ der Patienten $(n=46)$ wiesen einen oder mehrere Suizidversuche in der Vorgeschichte auf (30\% keinen; 14\% nicht eruierbar). Bei 30\% der Patienten war ein Suizidversuch zu beobachten, 54\% haben 2-3 mal versucht sich das Leben zu nehmen, $13 \%$ probierten es mehr als dreimal; $2 \%$ der Personen gehörten zu der Gruppe die mehr als zehnmal versucht hat sich umzubringen,

\section{Abbildung 5 Suizidversuche in der Anamnese}

Referenzgruppe: Patienten, die mindestens einen Suizidversuch unternommen hatten $(\mathrm{n}=46$ Patienten)



\subsubsection{Dokumentation der diagnostischen Kriterien nach ICD-10 und DSM-IV}

Für die korrekte Diagnose müssen mindestens fünf der zehn diagnostischen Kriterien der Borderline-Persönlichkeitsstörungen erfüllt sein. Es galt zu prüfen, inwiefern bei den gestellten Diagnosen die respektive Kriterien aufgrund der Dokumentation erfüllt waren. In der vorliegender Studie erfüllten 93\% der Patienten die Kriterien nach ICD10 (WHO 1993); nach DSM-IV (APA 1994) waren die Klassifikationskriterien bei 95\% der Patienten dokumentiert. 


\section{Abbildung 6 Dokumentation der einzelnen diagnostischen Kriterien nach ICD-10 (WHO 1993) ( $N=87$ Patienten)}

Störung und Unsicherheit bezüglich Selbstbild, Zielen und innerer Präferenzen $(n=80)$

Deutliche Tendenz, unerwartet und ohne Berücksichtigung der Konsequenzen zu handeln $(n=77)$

Neigung zu Ausbrüchen von Wut oder Gewalt mit Unfähigkeit zur Kontrolle explosiblen Verhaltens $(n=63)$

Neigung, sich auf intensive, aber instabile Beziehungen einzulassen, oft mit der Folge emotionaler Krisen $(n=60)$

Übertriebene Bemühungen, das Verlassenwerden zu vermeiden $(n=60)$

Deutliche Tendenz zu Streitereien und Konflikten mit anderen, v.a., wenn impulsive Handlungen unterbunden werden $(n=46)$

Schwierigkeiten in der Beibehaltung von Handlungen, die nicht unmittelbar belohnt werden $(n=45)$

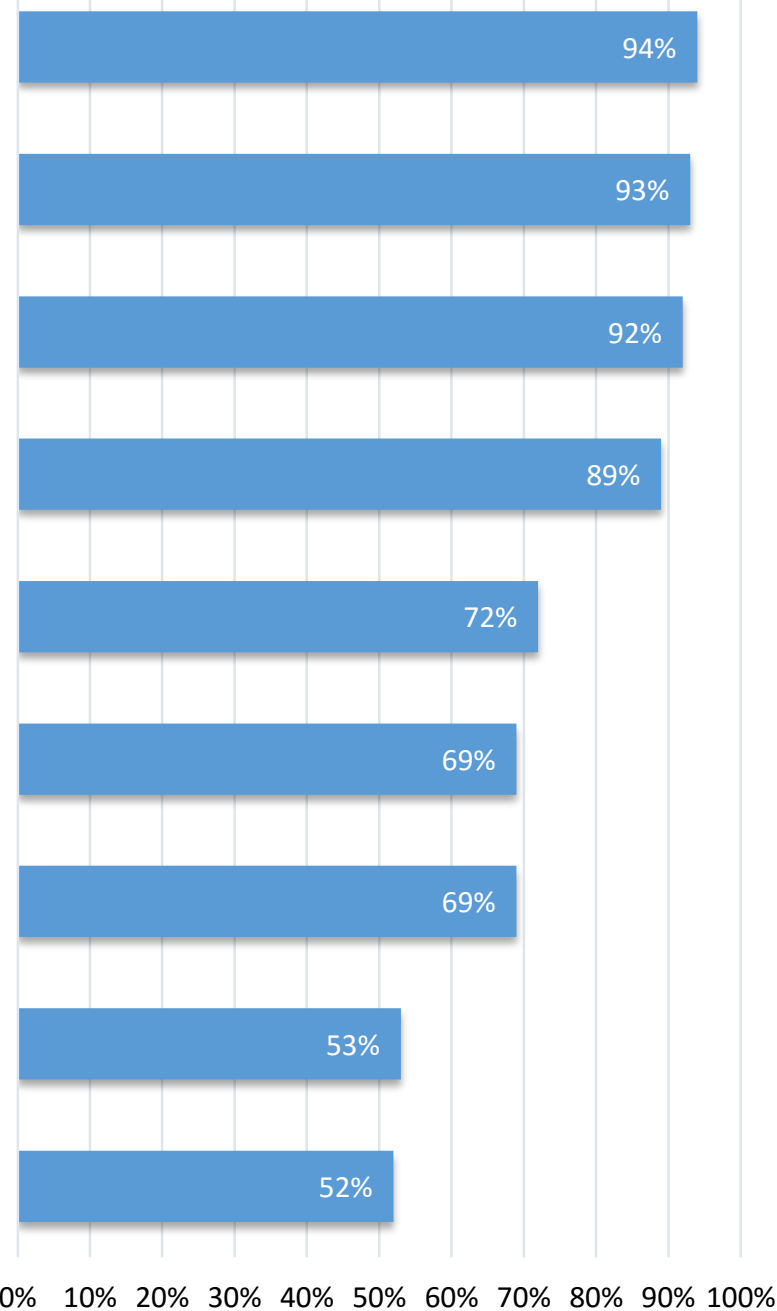

$\begin{array}{lllllllllll}0 \% & 10 \% & 20 \% & 30 \% & 40 \% & 50 \% & 60 \% & 70 \% & 80 \% & 90 \% & 100 \%\end{array}$

Prozent der Patienten

Aufgrund der Ähnlichkeit beider Klassifikationssysteme beschränken sich die Ergebnisse im Weiteren auf das ICD-10 Klassifikationssystem. Der Großteil der Patienten (über 90\%) erfüllte das Kriterium Affektive Instabilität. Alleinsein zu verhindern und chronisches Gefühl der Leere waren bei der Mehrheit der Patienten (94\%) dokumentiert. 93\% hatten Probleme, ihre Wut oder ihren Ärger zu kontrollieren bzw. zeigten Suiziddrohungen/selbstverletzendes Verhalten, 69\% der Patienten hatten Probleme mit instabilen intensiven zwischenmenschlichen Beziehungen. 


\section{Abbildung 7 Dokumentation der einzelnen diagnostischen Kriterien nach DSM-IV (APA 1994)} (N=87 Patienten)

\section{Dokumentation der einzelnen diagnostischen Kriterien nach DSM-IV}

(APA 1994)

( $N=87$ Patienten)

Diagnostische Kriterien insgesamt (> 5; n=83)

Affektive Instabilität mit starken Stimmungsschwankungen $(n=85)$

Häufig Suiziddrohungen, -andeutungen, -versuche oder Selbstverletzungen $(n=80)$

Identitätsstörung: deutliches und dauerhaft bestehendes unbeständiges Selbstbild $(n=77)$

Chronische Leeregefühle $(n=73)$

Unbeständige und intensive zwischenmenschliche Beziehungen mit einem Wechsel zwischen den

Extremen der Idealisierung und Abwertung $(n=67)$

Übermäßige, starke Wut oder Unfähigkeit, die Wut zu kontrollieren $(n=66)$

Verzweifeltes Bemühen, ein reales oder vorgestelltes Verlassenwerden zu vermeiden $(n=61)$

Impulsivität bei mindenstens 2 potentiell selbst schädigenden Aktivitäten $(n=40)$

Vorübergehende, stressbedingte Wahnvorstellungen oder dissoziative Symptome $(n=15)$

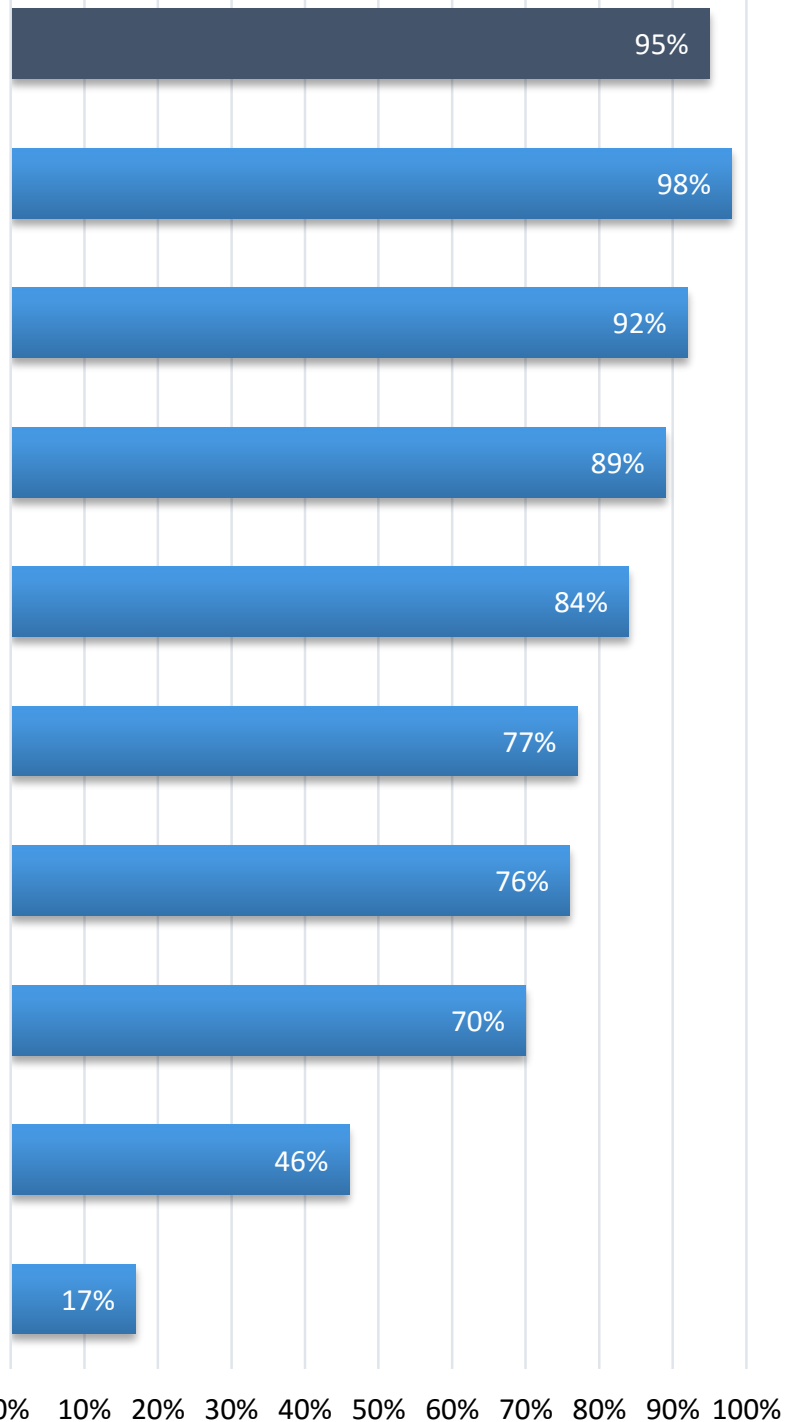

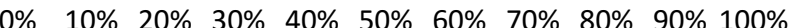

Prozent der Patienten

Andere Kriterien waren weniger häufig dokumentiert. So waren stressbedingte Wahnvorstellungen oder dissoziative Symptome, die ein Kriterium im DSM-IV darstellen, bei $17 \%$ der Patienten eruierbar. 


\subsection{Aufenthaltscharakteristika}

\subsubsection{Aufnahmeanlässe}

Hierbei waren Mehrfachnennungen möglich. Häufigster Aufnahmeanlass war eine affektive Störung (96\%); parasuizidale Handlungen standen bei 94\% der Patienten im Vordergrund. Suizidalität hatten $85 \%$ der Patienten. Eine Angststörung und Selbstverletzung waren weitere Gründe bei mehr als der Hälfte aller Aufnahmen. Ferner führten Intoxikationen in nicht suizidaler Absicht, Entzugswünsche, Arbeitsplatzverlust oder geplante Psychotherapien nicht selten zur stationären Aufnahme.

\section{Abbildung 8 Aufnahmeanlass für den stationären Aufenthalt} ( $\mathrm{N}=140$ Aufenthalte)

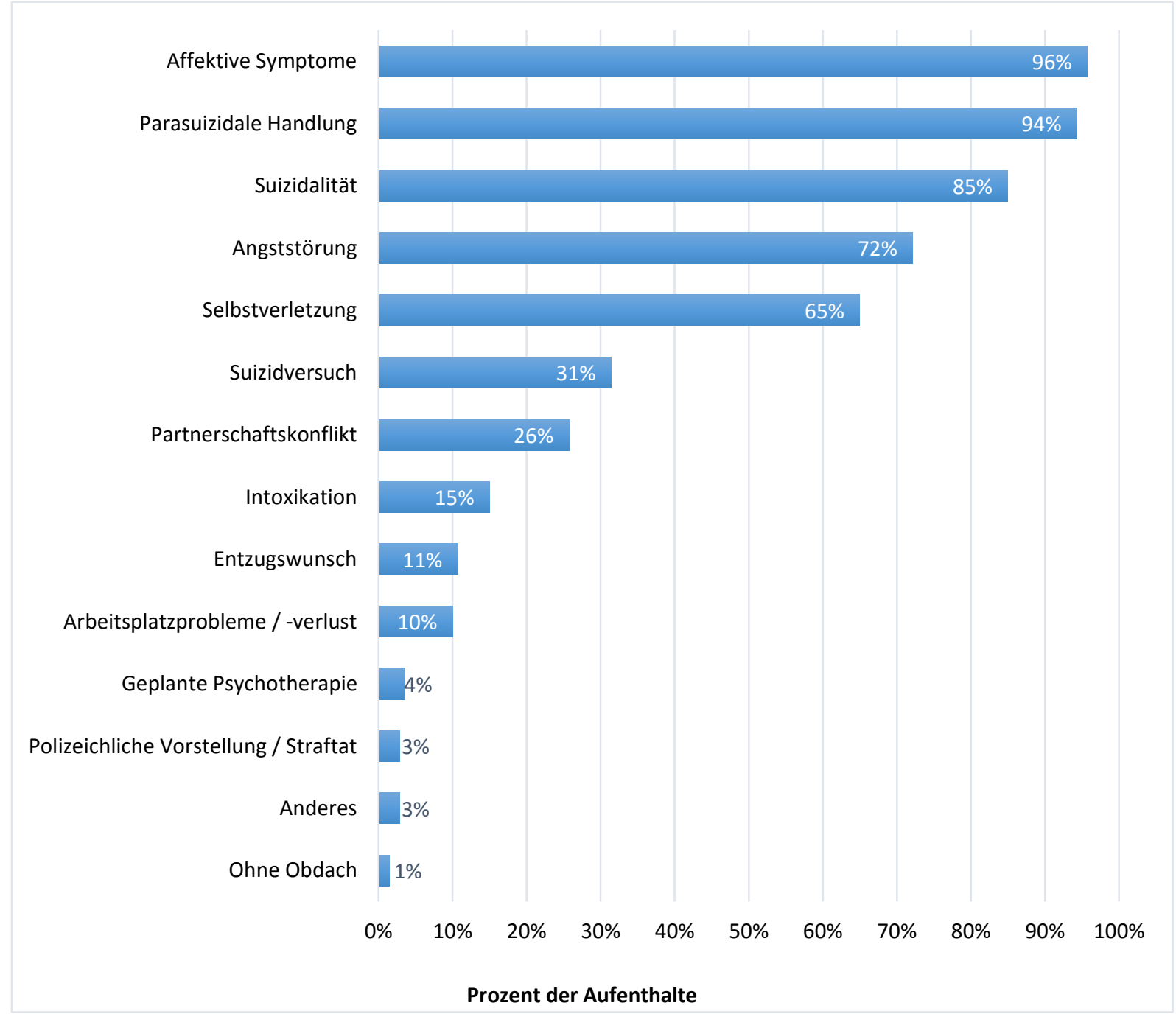




\subsubsection{Dauer der stationären Behandlung bei verschiedenen Behandlungsmodi}

Die durchschnittliche Dauer eines stationären Aufenthalts im Untersuchungszeitraum 2008-2012 betrug 22,7 Tage (SD 34,6). Bei differenziertere Betrachtung ergaben sich dezente Unterschiede je nach Aufnahmeanlass. Kriseninterventionen, die einen Großteil der Aufenthalte bedingten, dauerten mit durchschnittlich 24,1 Tage (SD 37,4) etwas länger als Psychotherapien mit 20,3 Tagen (SD 24,8) im Schnitt. Suchtbehandlungen waren mit im Mittel 15,6 Tagen (SD 13,0) nominell deutlich kürzer als Kriseninterventionen.

\section{Abbildung 9 Dauer der stationären Behandlung bei verschiedenen Behandlungsmodi} ( $N=140$ Aufenthalte)

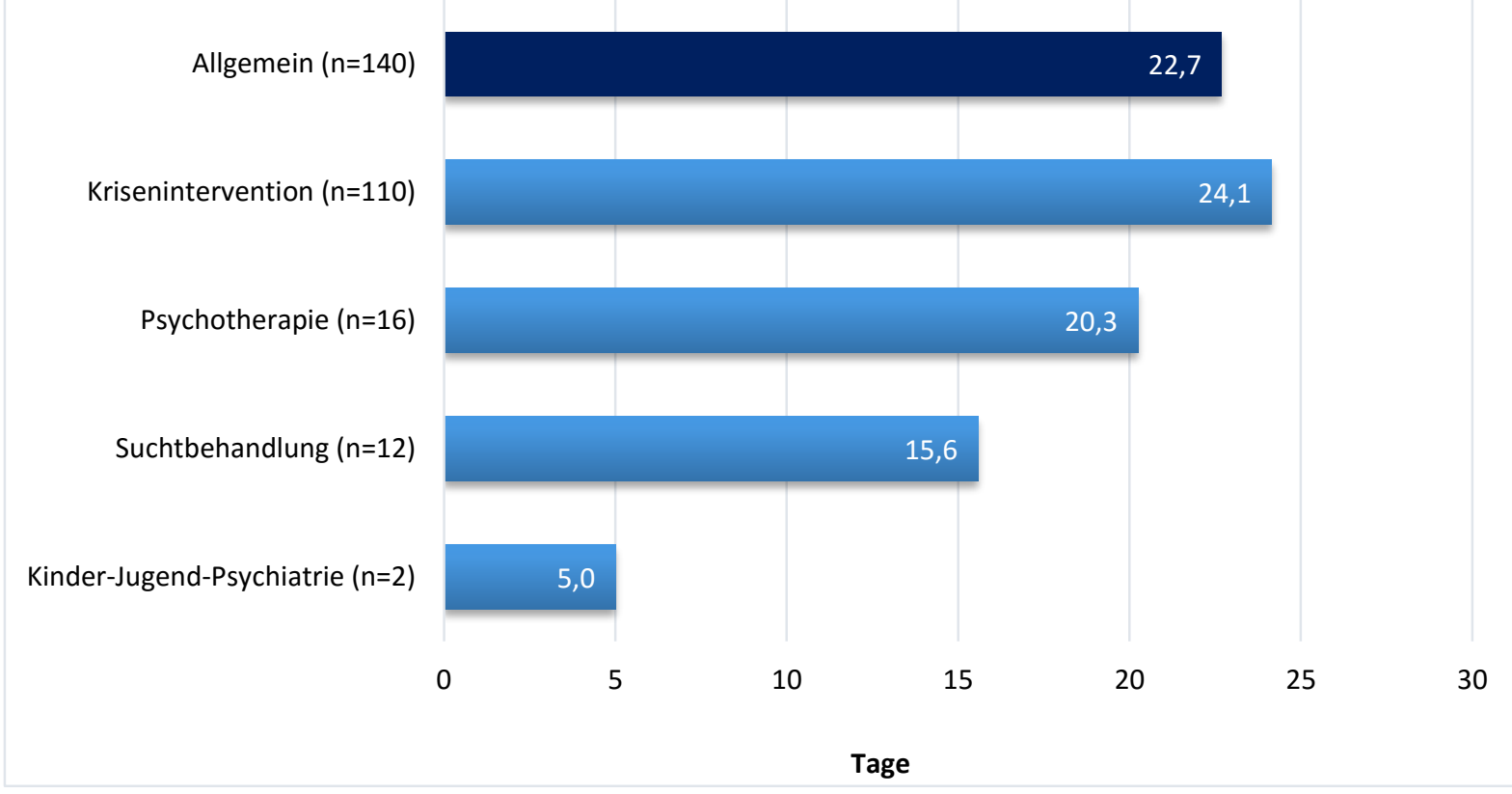

\subsection{Psychopharmakotherapie}

\subsubsection{Psychopharmakaanamnese über den gesamten Behandlungszeitraum}

Speziell zur Behandlung der EIPS / BPS ist bisher kein Medikament zugelassen, so dass hier nur im Rahmen von Off-Label-Use (zulassungsüberschreitender Einsatz von Arzneimitteln) oder komorbider psychischer Störungen symptomorientiert therapiert wird. Angesichts der aktuellen vielfältigen Symptomatik und der Heterogenität des 
Störungsbildes richtet sich die Wahl des einzusetzenden Medikaments danach, welche Symptomatik aktuell im Vordergrund steht und das stärkste Leiden verursacht. In der Abbildung wird ersichtlich, dass $67 \%$ der Patienten schon bei der Aufnahme mit Psychopharmaka behandelt wurden, während der stationären Behandlung waren es 94\%. Nach den Unterlagen erhielten ein eher geringer Anteil von Patienten (10\%) eine Reservemedikation.

\section{Abbildung 10 Einnahme von Psychopharmaka bei Aufnahme, während stationärer} Behandlung und als Reserve

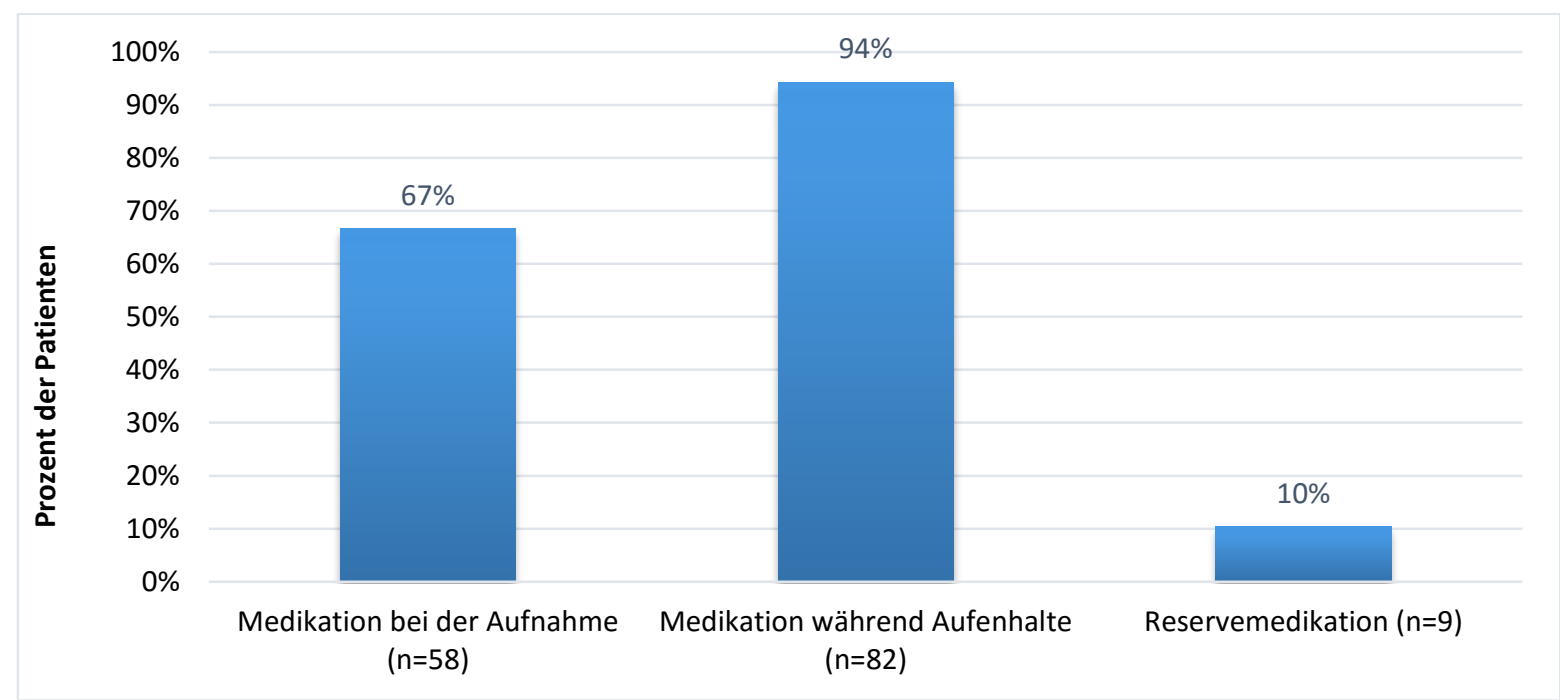

\subsubsection{Häufigkeitsverteilung verschiedener Substanzgruppen}

\subsubsection{Hauptgruppen}

Im Folgenden wird die Häufigkeit der Gabe verschiedener Substanzen (Antipsychotika/Neuroleptika, Antidepressiva, Hypnotika und Sedativa, Stimmungsstabilisierer/Mood Stabilizer sowie Entwöhnungsmittel) bei Aufnahme in der Klinik dargestellt. 
Abbildung 11 Gabe verschiedener Psychopharmakahauptgruppen bei der Aufnahme

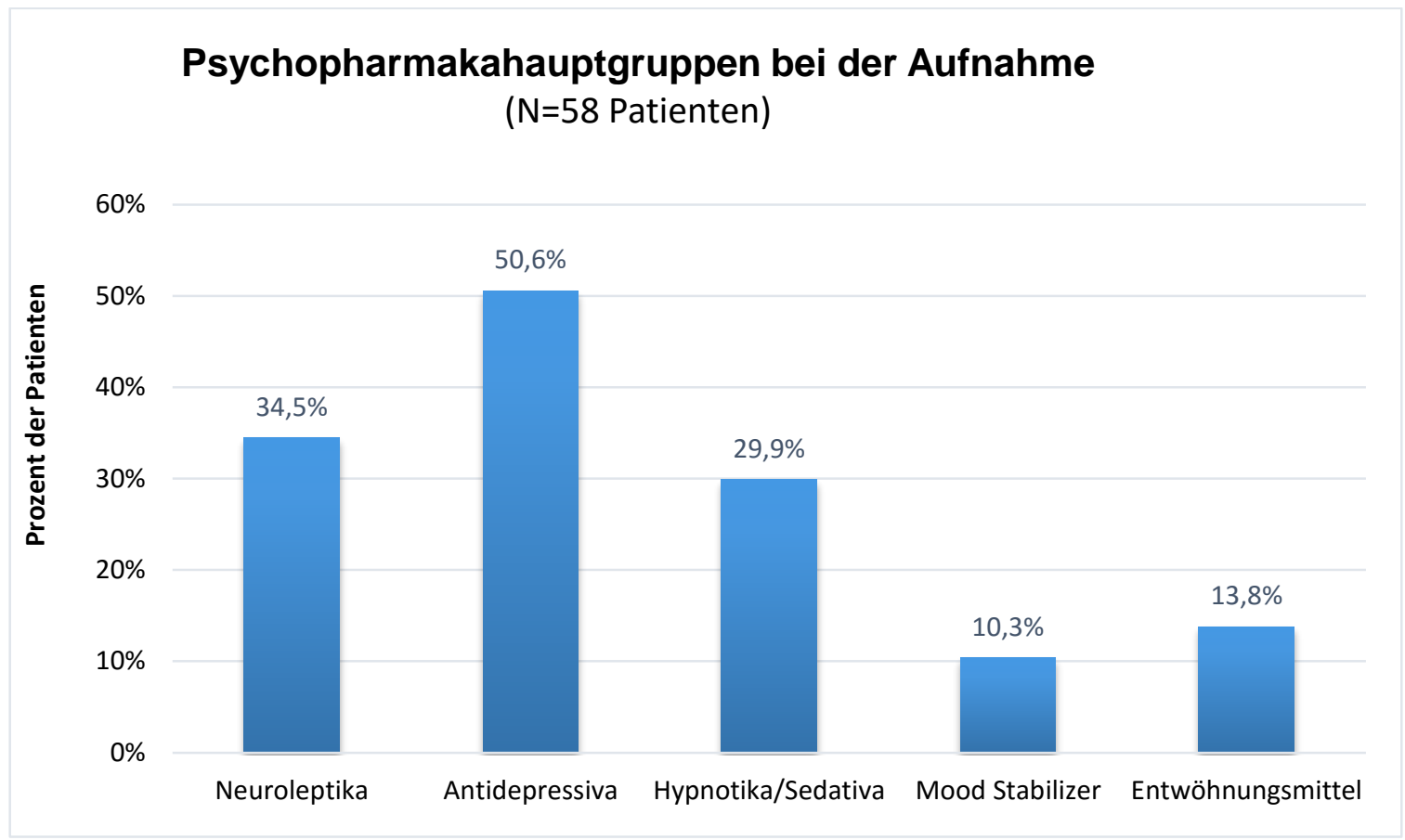

Unter einzeln verordneten Substanzen stehen Antidepressiva im Vordergrund; 50,6\% der Patienten hatten eine regelmäßige Verordnung von Substanzen dieser Medikamentengruppe zum Aufnahmetag. An der zweiten Stelle rangierten mit 34,5\% die Antipsychotika/Neuroleptika, 29,9\% haben Hypnotika bekommen. Mood Stabilizer hatten 10,3\% der Patienten in Ihrer Verordnung, Entwöhnungsmittel 13,8 \%.

\section{Abbildung 12 Gabe verschiedener Psychopharmakahauptgruppen bei der Entlassung}

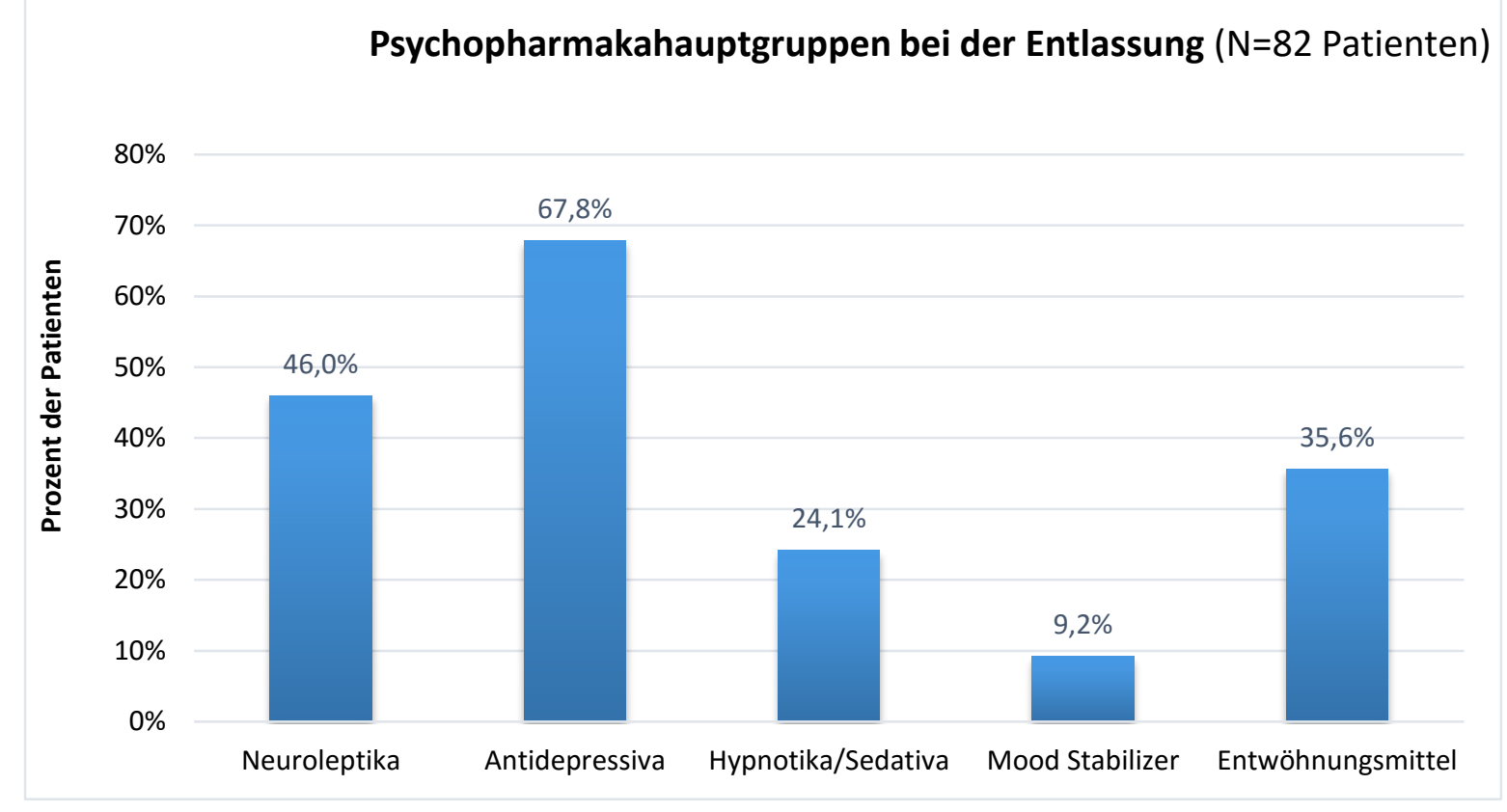


Bei der Entlassungsmedikation sind veränderte Ergebnisse zu sehen. Der Anteil von Antidepressiva (50,6\% auf 67,8\%) ist über den Aufenthalt angestiegen. Antipsychotika wurden nominell über den Aufenthalt gesteigert (34,5\% auf 46\%). Im Unterschied zur Aufnahmemedikation wurden etwas weniger Hypnotika und Mood Stabilizer verordnet. Auffällig ist ein deutlicher Anstieg der Verordnung von Entwöhnungsmitteln von 13,8\% auf $35,6 \%$. Hierbei handelt es sich, wie weiter unten beschrieben, hauptsächlich um die Verschreibung von Naltrexon.

Betrachtet man nur die Patienten als Referenzgruppe, die überhaupt eine Medikation bei Entlassung bekamen $(n=82)$, änderte sich die prozentuale Verteilung wie unten beschrieben.

\subsubsection{Untergruppen}

a) Antipsychotika/Neuroleptika

Bei der Aufnahme und während der stationären Behandlung bekamen die meisten Patienten atypische Neuroleptika, bzw. Antipsychotika der zweiten Generation, oder niederpotente Neuroleptika (60\%). Die Hälfte dieser Patienten hatte niederpotente Neuroleptika eingenommen. Es wurden keine klassischen hochpotenten Neuroleptika wie z.B. Haloperidol gegeben.

\section{Abbildung 13 Einnahme von Neuroleptika bei der Aufnahme und während stationärer Behandlung; ( $\mathrm{N}=30$ Patienten). Mehrfachnennungen möglich}

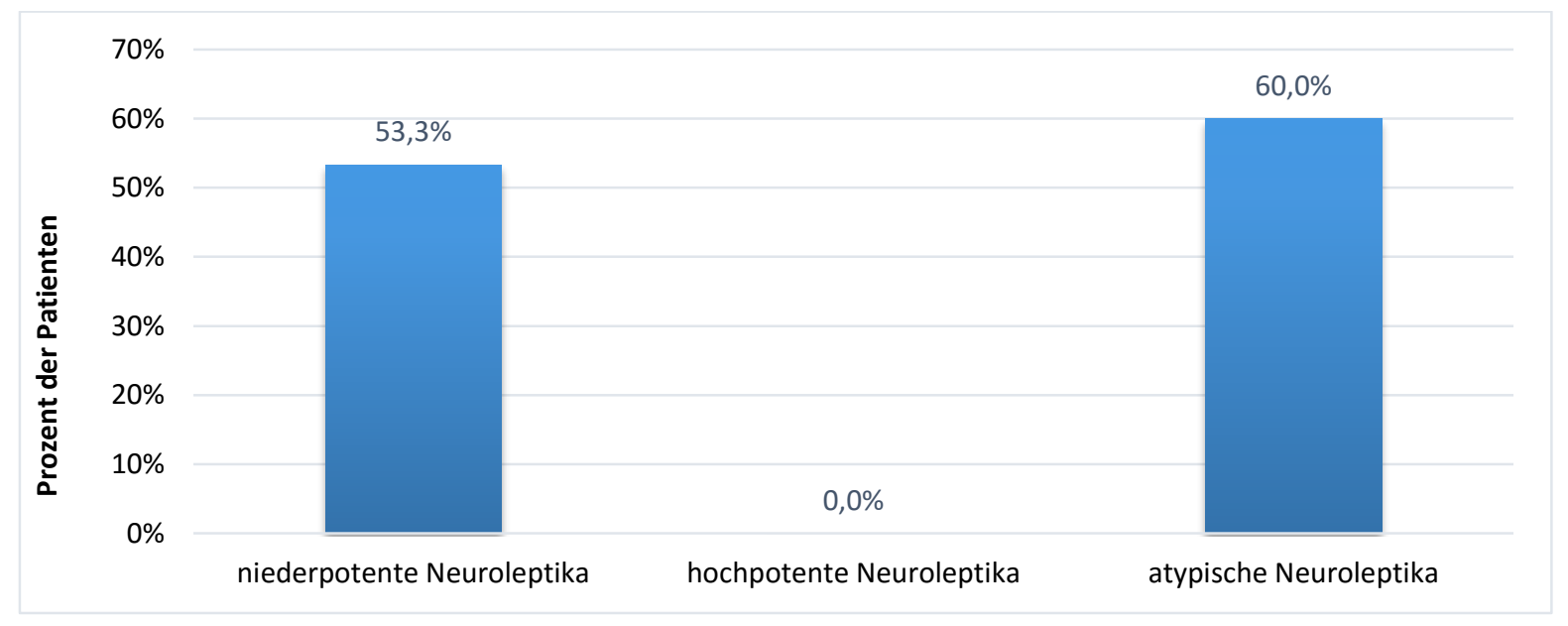

Betrachtet man die Häufigkeitsverteilung bei der Entlassungsmedikation, ergibt sich ein ähnliches Ergebnis wie bei der Aufnahmemedikation. Einziger Unterschied ist, 
dass $5 \%$ der Patienten bei Entlassung klassische hochpotente Neuroleptika einnahmen, die sie zu Beginn nicht bekamen.

Abbildung 14 Einnahme von Neuroleptika bei der Entlassung; ( $\mathrm{N}=40$ Patienten). Mehrfachnennungen möglich

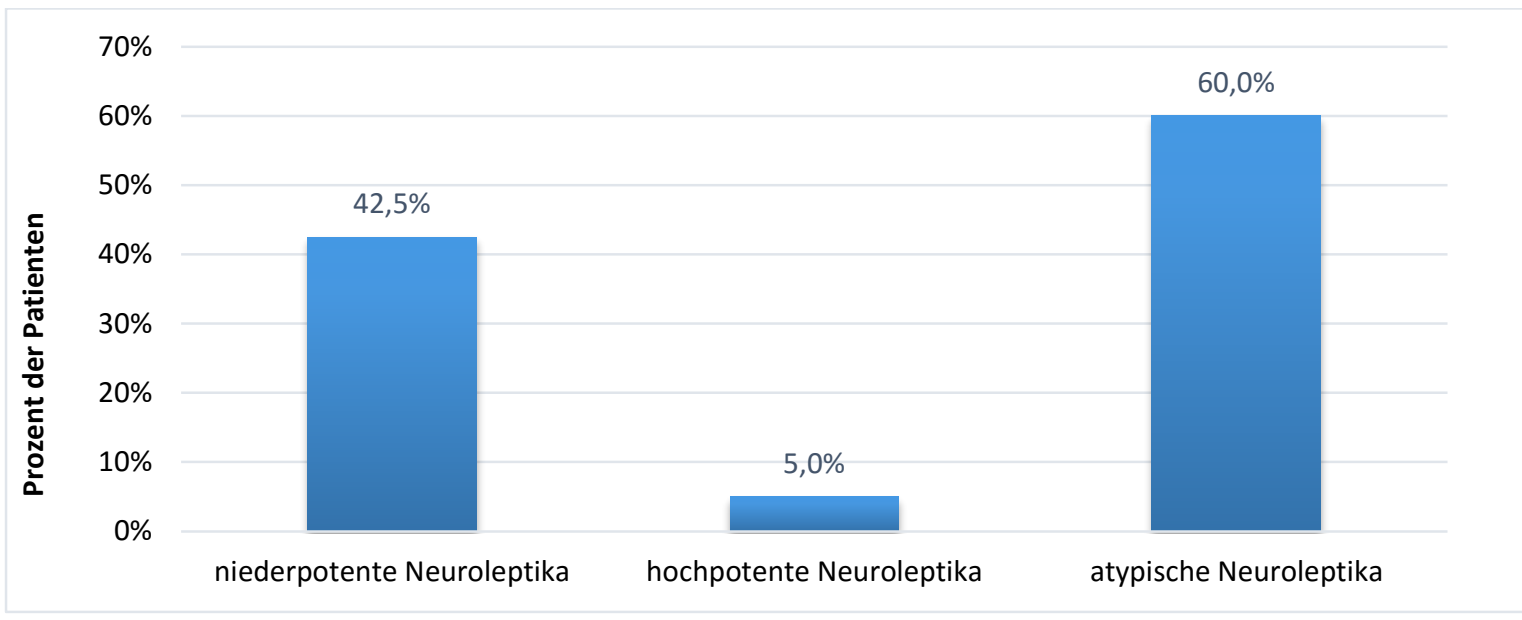

b) Antidepressiva

Am häufigsten eingesetzt wurde die medikamentöse Therapie mit den selektiven Serotonin-Wiederaufnahme-Hemmern (SSRI). Atypische Antidepressiva (worunter hier z.B. das Mirtazapin gezählt wurde) bekamen ein Drittel der mit Antidepressiva behandelten Patienten. 13\% erhielten Tri- und Tetrazyklische Antidepressiva. MAOHemmer waren von geringerer Bedeutung (2,3\%).

\section{Abbildung 15 Einnahme von Antidepressiva bei der Aufnahme und während stationärer Behandlung; ( $\mathrm{N}=44$ Patienten)}

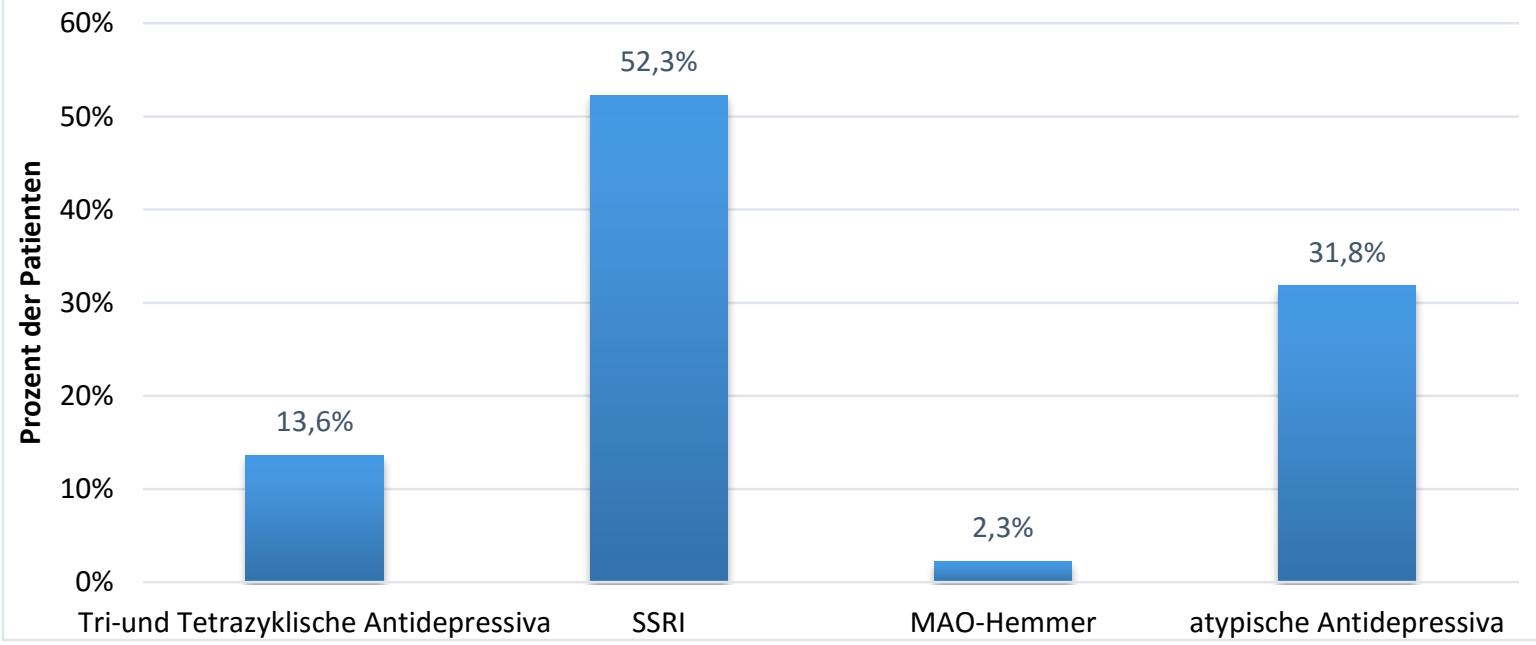


Zur Häufigkeitsverteilung bei der Entlassungsmedikation ergibt sich folgendes, im Wesentlichen ähnliches Ergebnis.

Abbildung 16 Gabe von Antidepressiva bei Entlassung; (N=59 Patienten)

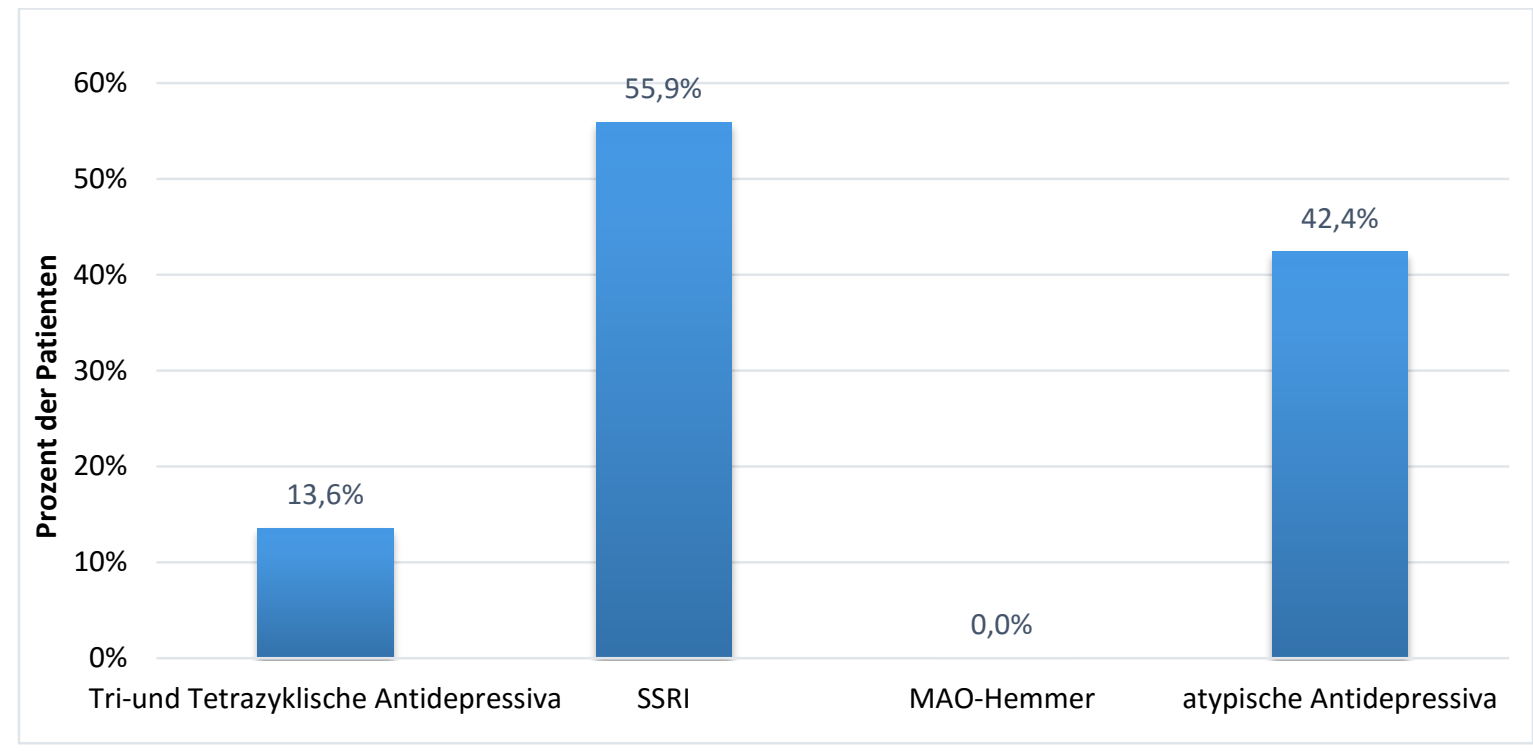

Im Vergleich zur Aufnahme wurden zur Entlassung häufiger atypische Antidepressiva gegeben, MAO-Hemmer wurde während der Behandlung grundsätzlich abgesetzt. Die Häufigkeit der Verordnung von SSRI und Tri- bzw. Terazyklika war annähernd konstant, es zeigte sich kein signifikanter Unterschied.

c) Hypnotika und Sedativa

Bei der Hypnotika und Sedativa wurden mit Abstand am häufigsten Benzodiazepine $(22,3 \%)$ gegeben. Non-Benzodiazepine hatte ein Viertel der mit Hypnotika/Sedativa therapierten Patienten schon einmal verordnet bekommen.

\subsubsection{Wirkstoffe}

Im folgenden Kapitel wird die Häufigkeit der Gabe verschiedener PsychopharmakaWirkstoffe in der Untersuchungsgruppe in Prozent bei Entlassung dargestellt. Als weiterer Parameter werden die Durchschnittsdosis sowie die Dosisrange (Spannweite) angegeben. Anmerkungen zu Dosierungen erscheinen schwierig, da es sich bei den ermittelten Werten um Durchschnittsdosen handelt, die durch Ein- und Ausschleichen 
der Medikamente nicht die tatsächliche konstante therapeutische Dosis widerspiegeln können.

Bei der Entlassung wurden unter den niederpotenten Neuroleptika vorwiegend Prothipendyl (8\%) gegeben. Die am häufigsten gegebenen atypischen Neuroleptika war Quetiapin (19,5\%).

Betrachtet man die Antidepressiva genauer, wird ersichtlich, dass unter innen vorwiegend SSRI wie Sertralin (12,6\%), Escitalopram $(11,5 \%)$ und Fluoxetin $(9,2 \%)$ verabreicht wurden. Bei atypischen Antidepressiva stand Mirtazapin $(18,4)$ eindeutig im Vordergrund. MAO-Hemmer wurde nicht verordnet.

Unter den Hypnotika und Sedativa dominieren Diazepam (10,3\%) und Lorazepam $(9,2 \%)$.

Das am häufigsten verordnete Medikament unter den Entwöhnungsmitteln war Naltrexon (35\%).

In folgender Tabelle wird die durchschnittliche Psychopharmakotherapie bei der Entlassung aufgelistet ( $\mathrm{N}=142$ Aufenthalte). 
Tabelle 6 Entlassungsmedikation

\begin{tabular}{|c|c|c|c|c|c|c|c|}
\hline & \begin{tabular}{|l} 
Prozent der \\
Patienten \\
mit dem \\
Pharmakon \\
$2008-2012$
\end{tabular} & $\begin{array}{l}\text { Prozent der } \\
\text { Patienten } \\
\text { mit dem } \\
\text { Pharmakon } \\
1996-2004\end{array}$ & $\begin{array}{l}\text { Patienten- } \\
\text { anzahl } \\
(\mathrm{N}=87 \\
\text { Patienten) }\end{array}$ & $\begin{array}{l}\text { Aufenthalts- } \\
\text { anzahl } \\
(\mathrm{N}=142)\end{array}$ & $\begin{array}{l}\text { Durch- } \\
\text { schnitts- } \\
\text { dosis } \\
\text { (mg) }\end{array}$ & $\begin{array}{l}\text { Dosis- } \\
\text { range } \\
\text { (mg) }\end{array}$ & $\begin{array}{l}\text { Durch- } \\
\text { schnitts- } \\
\text { dosis (mg) } \\
1996-2004\end{array}$ \\
\hline \multicolumn{8}{|l|}{ Neuroleptika } \\
\hline \multicolumn{8}{|c|}{ Niederpotente Neuroleptika } \\
\hline Perazin & $1,1 \%$ & $9,9 \%$ & 1 & & & & \\
\hline Pipamperon & $1,1 \%$ & $9,2 \%$ & 1 & & & & \\
\hline Promethazin & $3,4 \%$ & $9,2 \%$ & 3 & & & & \\
\hline Levomepromazin & $1,1 \%$ & $6,3 \%$ & 1 & & & & \\
\hline Chlorprothixen & $2,3 \%$ & $4,9 \%$ & 2 & & & & \\
\hline Prothipendyl & $8,0 \%$ & $4,9 \%$ & 7 & 11 & 96,3 & $40-160$ & \\
\hline Melperon & $2,3 \%$ & $4,2 \%$ & 2 & & & & \\
\hline Sulpirid & $1,1 \%$ & $3,5 \%$ & 1 & & & & \\
\hline \multicolumn{8}{|c|}{ Atypische Neuroleptika } \\
\hline Ariprazol & $4,6 \%$ & & 4 & & & & \\
\hline Ziprasidon & $1,1 \%$ & & 1 & & & & \\
\hline Olanzapin & $1,1 \%$ & $9,9 \%$ & 1 & & & & \\
\hline Quetiapin & $19,5 \%$ & $7,0 \%$ & 17 & 36 & 238,3 & $75-700$ & 341,4 \\
\hline Risperidon & $1,1 \%$ & $3,5 \%$ & 1 & & & & \\
\hline \multicolumn{8}{|l|}{ Antidepressiva } \\
\hline \multicolumn{8}{|c|}{ Tri- und Tetrazyklische Antidepressiva } \\
\hline Trimipramin & $3,4 \%$ & $14,8 \%$ & 3 & & & & \\
\hline Doxepin & $3,4 \%$ & $12,7 \%$ & 3 & & & & \\
\hline Amitriptylin & $2,3 \%$ & $4,9 \%$ & 2 & & & & \\
\hline \multicolumn{8}{|l|}{ SSRI } \\
\hline Duloxetin & $1,1 \%$ & & 1 & & & & \\
\hline Citalopram & $3,4 \%$ & $13,4 \%$ & 3 & & & & \\
\hline Fluoxetin & $9,2 \%$ & $10,6 \%$ & 8 & 18 & 58,3 & $20-400$ & 26,7 \\
\hline Sertralin & $12,6 \%$ & $7,7 \%$ & 11 & 10 & 80 & $50-100$ & 123,5 \\
\hline Paroxetin & $1,1 \%$ & $4,9 \%$ & 1 & & & & \\
\hline Escitalopram & $11,5 \%$ & $0,7 \%$ & 10 & 16 & 21,8 & $20-40$ & 20 \\
\hline \multicolumn{8}{|c|}{ Atypische Antidepressiva } \\
\hline Mirtazapin & $18,4 \%$ & $14,1 \%$ & 16 & 23 & 14,6 & $8-60$ & 40,6 \\
\hline Venlafaxin & $10,3 \%$ & $4,2 \%$ & 9 & 18 & 143,7 & $75-225$ & 153,5 \\
\hline \multicolumn{8}{|c|}{ Hypnotika und Sedativa } \\
\hline \multicolumn{8}{|l|}{ Benzodiazepine } \\
\hline Diazepam & $10,3 \%$ & $11,3 \%$ & 9 & 20 & 18,7 & $5-80$ & 19,7 \\
\hline Lorazepam & $9,2 \%$ & $8,5 \%$ & 8 & 9 & 2,6 & $1-5$ & 2,8 \\
\hline Flunitrazepam & $2,3 \%$ & $1,4 \%$ & 2 & & & & \\
\hline Lormetazepam & $1,1 \%$ & $0,7 \%$ & 1 & & & & \\
\hline \multicolumn{8}{|c|}{ Nonbenzodiazepine } \\
\hline Zolpidem & $2,3 \%$ & $4,2 \%$ & 2 & & & & \\
\hline Zoplicon & $3,4 \%$ & $1,4 \%$ & 3 & & & & \\
\hline
\end{tabular}




\begin{tabular}{|l|l|l|l|l|l|l|l|}
\hline & $\begin{array}{l}\text { Prozent der } \\
\text { Patienten } \\
\text { mit dem } \\
\text { Pharmakon } \\
2008-2012\end{array}$ & $\begin{array}{l}\text { Prozent der } \\
\text { Patienten } \\
\text { mit dem } \\
\text { Pharmakon } \\
1996-2004\end{array}$ & $\begin{array}{l}\text { Patienten- } \\
\text { anzahl } \\
(\mathrm{N}=87 \\
\text { Patienten) }\end{array}$ & $\begin{array}{l}\text { Aufenthalts- } \\
\text { anzahl } \\
(\mathrm{N}=142)\end{array}$ & $\begin{array}{l}\text { Durch- } \\
\text { schnitts- } \\
\text { dosis } \\
\text { (mg) }\end{array}$ & $\begin{array}{l}\text { Dosis- } \\
\text { range } \\
(\mathrm{mg})\end{array}$ & $\begin{array}{l}\text { Durch- } \\
\text { schnittsdo- } \\
\text { sis (mg) } \\
1996-2004\end{array}$ \\
\hline Mood Stabilizer \\
\hline Carbamazepin & $3,4 \%$ & $20,4 \%$ & 3 & & & & \\
\hline Valproat & $5,7 \%$ & $2,8 \%$ & 5 & 7 & 728,5 & $\begin{array}{l}300- \\
1200\end{array}$ & 687,5 \\
\hline Gabapentin & $1,1 \%$ & $1,4 \%$ & 1 & & & & \\
\hline Lithium & $2,3 \%$ & $1,4 \%$ & 2 & & & & \\
\hline Lamotrigin & $4,6 \%$ & & 4 & & & & \\
\hline Entwöhnungsmittel & & & & & & \\
\hline Naltrexon & $35,6 \%$ & $6,3 \%$ & 31 & 48 & 73,9 & $50-200$ & 46,9 \\
\hline Disulfiram & $2,3 \%$ & $1,4 \%$ & 2 & & & & \\
\hline
\end{tabular}

Im Vergleich zu den Jahren 1996-2004 zeigte sich eine Abnahme von Verschreibungen nieder- und hochpotenter Neuroleptika wie Perazin $(1,1 \%)$, Pipamperon (1,1\%) und Promethazin (3,4\%). Dafür wurden häufiger atypische Neuroleptika, z.B. Quetiapin (19,5\%) und Ariprazol (4,6\%) verordnet.

Bei Betrachtung der Antidepressiva zeigt sich, dass im zweiten Zeitintervall die Zahlen der tri- und tetrazyklischen Antidepressiva wie Trimipramin und Doxepin um ein Viertel zurückgegangen sind. Unter den SSRIs wurde im zweiten Zeitintervall doppelt so häufig Sertalin, Paroxetin und Escitalopram als in den Jahren 1996-2004 verschrieben. Bei den atypischen Antidepressiva dominierte im Vergleich Venlafaxin. Die Gabe von Mirtazapin ist leicht angestiegen. In den letzten Jahren wurde das Medikament hauptsächlich als Antidepressivum angeordnet, heute gibt man es auch als schlaff förderndes Mittel.

Betrachtet man die Häufigkeitsverteilung in beiden Gruppen bei Hypnotika und Sedativa, wird ersichtlich, dass Diazepam nach wie vor am häufigsten verabreicht wurde. Unter den Nonbenzodiazepinen dominierte Zoplicon (3,4\%).

Verordnungen von Mood- Stabilizern sind im zweiten Beobachtungsintervall (20082012) zurückgegangen. Der am häufigsten verabreichte Mood- Stabilizer Carbamazepin $(3,4 \%)$ wurde bei der Entlassung genauso häufig verabreicht, wie Zoplicon.

Vergleicht man die Entlassungsmedikation zwischen beiden Untersuchungsgruppen, so wurde im zweiten Beobachtungsintervall fünf Mal häufiger das Entwöhnungsmittel Naltrexon verschrieben als in den Jahren zuvor. 


\subsection{Vergleich der Ergebnisse mit der Studie des Zeitraums 1996 - 2004}

Um Veränderungen bezüglich der Soziodemographie zu erkennen, wurden die Ergebnisse in zwei Zeitintervalle unterteilt, 1996 bis 2004, und 2008 bis 2012, so konnte man die Unterschiede über die Zeit verfolgen.

Abbildung 17 Durchschnittsalter ( $\mathrm{N}=87$ Patienten)

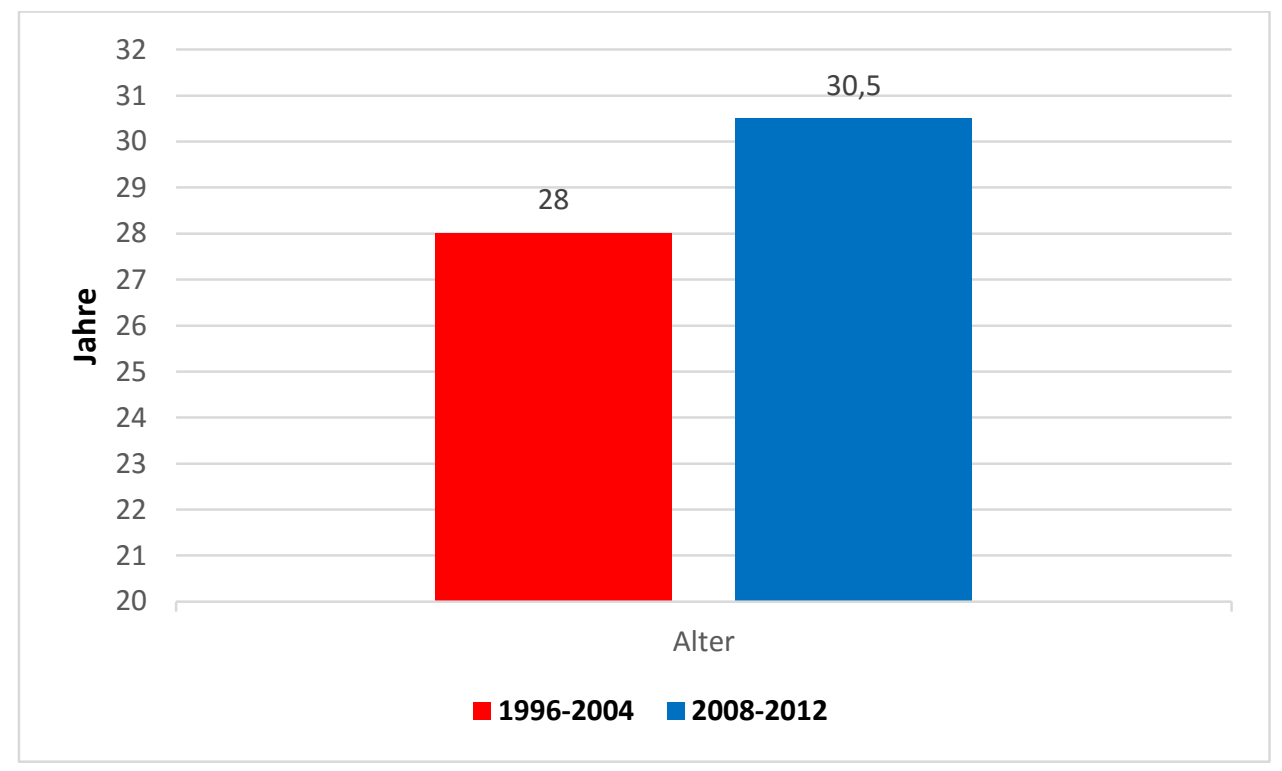

Beobachtet man beide Gruppen bezüglich des Alters ergab sich, dass die Patienten im zweiten Beobachtungsraum (20008-2012) um 2,5 Jahre älter waren, dieser Vergleich war allerdings nicht signifikant (t-test; zweiseitige Signifikanz $=0,052$, t-Wert $=1,955 ; \mathrm{df}=27,000)$. 


\section{Abbildung 18 Dauer der Aufenthalte:}

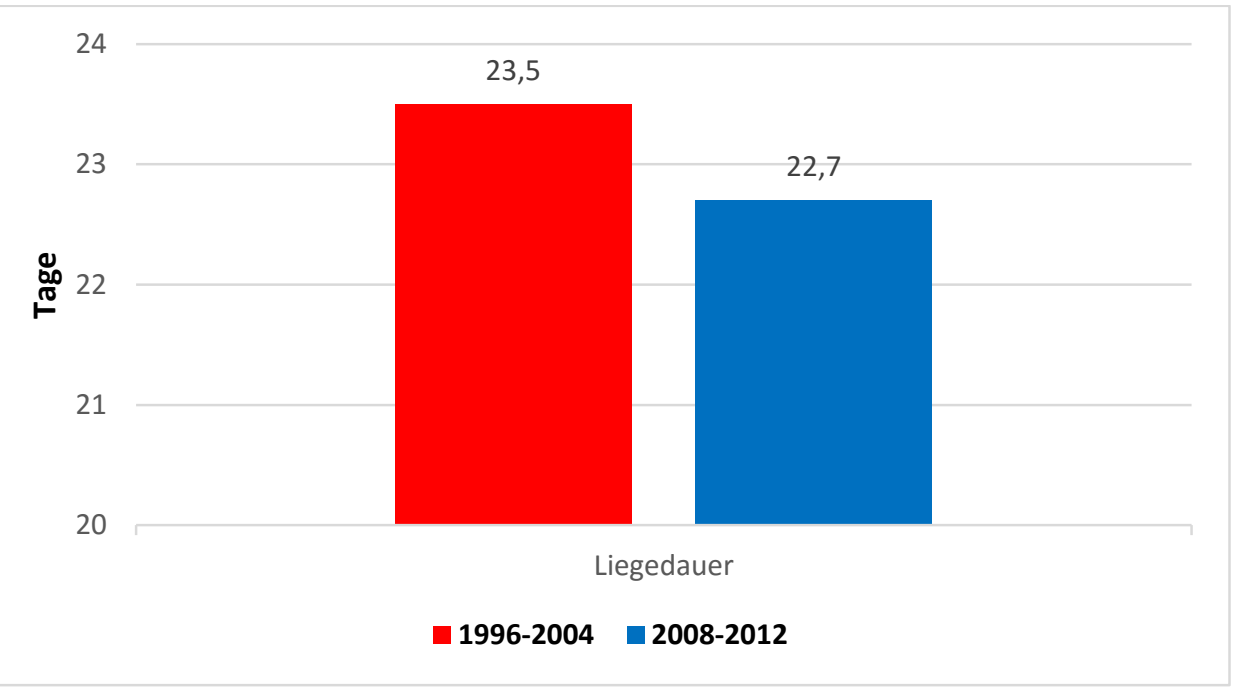

Hinsichtlich der Dauer eines Aufenthaltes zeigte sich zwischen 1996 und 2004 eine durchschnittliche Liegezeit von 23,5 Tagen, zwischen 2008-2012 waren es im Schnitt 22,7 Tage. Somit hat sich die Liegezeit um 0,8 verkürzt. Das Ergebnis ist demnach nicht signifikant (Signifikanz $=0,859$, t-Wert $=0,177, \mathrm{df}=227,000$ ) .

\subsubsection{Vergleich Soziodemographie}

Tabelle $7 \quad$ Vergleich Soziodemographie zwischen 1996 - 2004 und 2008 - 2012

\begin{tabular}{|c|c|c|c|c|c|c|c|}
\hline & Prozent & \begin{tabular}{|c|}
$\begin{array}{c}\text { Häufig- } \\
\text { keit }\end{array}$ \\
\end{tabular} & Prozent & $\begin{array}{c}\begin{array}{c}\text { Häufig- } \\
\text { keit }\end{array} \\
\text { k }\end{array}$ & Test-Wert & \begin{tabular}{|c}
$\begin{array}{c}\text { Freiheits- } \\
\text { grade }\end{array}$ \\
\end{tabular} & Signifikanz \\
\hline Studie in Jahren & \multicolumn{2}{|c|}{ 1996-2004 } & \multicolumn{2}{|c|}{ 2008-2012 } & & & \\
\hline Ehe & \multicolumn{2}{|c|}{$\mathrm{n}=139$} & \multicolumn{2}{|c|}{$\mathrm{n}=87$} & & & \\
\hline Nein & $66,2 \%$ & 92 & $83,9 \%$ & 73 & $\mathrm{chi}^{2}=8,527$ & $d f=1$ & $p=0.003$ \\
\hline $\mathrm{Ja}$ & $23,0 \%$ & 32 & $9,2 \%$ & 8 & $\mathrm{chi}^{2}=7,022$ & $d f=1$ & $p=0.008$ \\
\hline Geschieden & $10,8 \%$ & 15 & $6,9 \%$ & 6 & & & \\
\hline Partnerschaft & \multicolumn{2}{|c|}{$n=139$} & \multicolumn{2}{|c|}{$\mathrm{n}=87$} & & & \\
\hline $\mathrm{Ja}$ & $59,7 \%$ & 83 & $27,6 \%$ & 24 & \multirow{2}{*}{$\operatorname{chi}^{2}=22,153$} & \multirow{2}{*}{$d f=1$} & \multirow{2}{*}{$p<0.001$} \\
\hline Nein & $40,3 \%$ & 56 & $72,4 \%$ & 63 & & & \\
\hline $\begin{array}{l}\text { Häufiger } \\
\text { Partnerwechsel }\end{array}$ & \multicolumn{2}{|c|}{$n=131$} & \multicolumn{2}{|c|}{$n=70$} & & & \\
\hline Nein & $83,2 \%$ & 109 & $67,1 \%$ & 47 & \multirow{2}{*}{$\mathrm{chi}^{2}=6,775$} & \multirow{2}{*}{$d f=1$} & \multirow{2}{*}{$p=0.009$} \\
\hline $\mathrm{Ja}$ & $16,8 \%$ & 22 & $32,9 \%$ & 23 & & & \\
\hline Sexuelle Ausrichtung & \multicolumn{2}{|c|}{$\mathrm{n}=126$} & \multicolumn{2}{|c|}{$\mathrm{n}=87$} & & & \\
\hline Heterosexuell & $95,2 \%$ & 120 & $89,7 \%$ & 78 & \multirow{2}{*}{$\mathrm{chi}^{2}=2,450$} & \multirow{2}{*}{$d f=1$} & \multirow{2}{*}{$\mathrm{p}=0,117$} \\
\hline Homosexuell & $4,0 \%$ & 5 & $10,3 \%$ & 9 & & & \\
\hline Bisexuell & $0,8 \%$ & 1 & $0,0 \%$ & 0 & & nicht verwe & \\
\hline
\end{tabular}




\begin{tabular}{|c|c|c|c|c|c|c|c|}
\hline & Prozent & $\begin{array}{c}\begin{array}{c}\text { Häufig- } \\
\text { keit }\end{array} \\
\end{array}$ & Prozent & $\begin{array}{c}\text { Häufig- } \\
\text { keit }\end{array}$ & Test-Wert & \begin{tabular}{|c|}
$\begin{array}{c}\text { Freiheits- } \\
\text { grade }\end{array}$ \\
\end{tabular} & Signifikanz \\
\hline Wohnsituation & \multicolumn{2}{|c|}{$n=141$} & \multicolumn{2}{|c|}{$\mathrm{n}=87$} & & & \\
\hline Allein & $44,7 \%$ & 63 & $43,7 \%$ & 38 & $\mathrm{chi}^{2}=0,022$ & $d f=1$ & $\mathrm{p}=0,882$ \\
\hline Mit Partner & $22,7 \%$ & 32 & $21,8 \%$ & 19 & $\operatorname{chi}^{2}=0,023$ & $d f=1$ & $p=0,880$ \\
\hline Bei Eltern & $9,2 \%$ & 13 & $17,2 \%$ & 15 & $\mathrm{chi}^{2}=3,214$ & $d f=1$ & $p=0,073$ \\
\hline Betreutes Wohnen & $7,8 \%$ & 11 & $9,2 \%$ & 8 & $\operatorname{chi}^{2}=0,137$ & $\mathrm{df}=1$ & $p=0,711$ \\
\hline Stark wechselnd & $7,1 \%$ & 10 & $1,1 \%$ & 1 & \multicolumn{3}{|c|}{$\frac{1}{\text { nicht auswertbar }}$} \\
\hline Im Heim & $5,0 \%$ & 7 & $1,1 \%$ & 1 & \multicolumn{3}{|c|}{ nicht auswertbar } \\
\hline Kein fester Wohnsitz & $2,1 \%$ & 3 & $1,1 \%$ & 1 & \multicolumn{3}{|c|}{ nicht auswertbar } \\
\hline Anderes & $0,7 \%$ & 1 & $4,6 \%$ & 4 & \multicolumn{3}{|c|}{ nicht auswertbar } \\
\hline Tätigkeit & \multicolumn{2}{|c|}{$n=138$} & \multicolumn{2}{|c|}{$n=85$} & & & \\
\hline Arbeitslos & $45,7 \%$ & 63 & $32,9 \%$ & 28 & $\mathrm{chi}^{2}=3,518$ & $d f=1$ & $\mathrm{p}=0,061$ \\
\hline Student & $8,7 \%$ & 12 & $10,6 \%$ & 9 & $\mathrm{chi}^{2}=0,221$ & $d f=1$ & $p=0,638$ \\
\hline In ungelernter Arbeit & $8,7 \%$ & 12 & $8,2 \%$ & 7 & $\operatorname{chi}^{2}=0,014$ & $d f=1$ & $p=0,905$ \\
\hline Im gelernten Beruf & $8,0 \%$ & 11 & $8,2 \%$ & 7 & $\mathrm{chi}^{2}=0,005$ & $d f=1$ & $p=0,944$ \\
\hline Auszubildender & $7,2 \%$ & 10 & $14,1 \%$ & 12 & $\mathrm{chi}^{2}=2,793$ & $\mathrm{df}=1$ & $\mathrm{p}=0,095$ \\
\hline In Rente & $7,2 \%$ & 10 & $7,1 \%$ & 6 & $\mathrm{chi}^{2}=0,003$ & $\mathrm{df}=1$ & $p=0,958$ \\
\hline Hausfrau & $3,6 \%$ & 5 & $1,2 \%$ & 1 & \multicolumn{3}{|c|}{ nicht auswertbar } \\
\hline Schüler & $2,9 \%$ & 4 & $8,2 \%$ & 7 & \multicolumn{3}{|c|}{ nicht auswertbar } \\
\hline Erwerbsunfähig & $2,2 \%$ & 3 & $3,5 \%$ & 3 & \multicolumn{3}{|c|}{ nicht auswertbar } \\
\hline Arbeitsunfähig & $2,2 \%$ & 3 & $3,5 \%$ & 3 & \multicolumn{3}{|c|}{ nicht auswertbar } \\
\hline Häufig wechselnd & $1,4 \%$ & 2 & $1,2 \%$ & 1 & \multicolumn{3}{|c|}{ nicht auswertbar } \\
\hline In Umschulung & $1,4 \%$ & 2 & $1,2 \%$ & 1 & \multicolumn{3}{|c|}{ nicht auswertbar } \\
\hline Schulischer Abschluss & \multicolumn{2}{|c|}{$n=110$} & \multicolumn{2}{|c|}{$n=81$} & & & \\
\hline Hauptschule & $24,5 \%$ & 27 & $39,5 \%$ & 32 & $\mathrm{chi}^{2}=4,891$ & $\mathrm{df}=1$ & $\mathrm{p}=0,027$ \\
\hline Realschule & $27,3 \%$ & 30 & $30,9 \%$ & 25 & $\mathrm{chi}^{2}=0,293$ & $\mathrm{df}=1$ & $p=0,588$ \\
\hline Abitur & $34,5 \%$ & 38 & $18,5 \%$ & 15 & $\mathrm{chi}^{2}=5,977$ & $d f=1$ & $p=0,014$ \\
\hline Keine & $9,1 \%$ & 10 & $7,4 \%$ & 6 & $\mathrm{chi}^{2}=0,172$ & $\mathrm{df}=1$ & $\mathrm{p}=0,678$ \\
\hline Noch in Ausbildung & $0,0 \%$ & 0 & $2,5 \%$ & 2 & \multicolumn{3}{|c|}{ nicht auswertbar } \\
\hline Sonderschule & $4,5 \%$ & 5 & $1,2 \%$ & 1 & \multicolumn{3}{|c|}{ nicht auswertbar } \\
\hline Beruflicher Abschluss & \multicolumn{2}{|c|}{$\mathrm{n}=126$} & \multicolumn{2}{|c|}{$n=81$} & & & \\
\hline Keiner & $39,7 \%$ & 50 & $45,7 \%$ & 37 & $\mathrm{chi}^{2}=0,728$ & $\mathrm{df}=1$ & $p=0,394$ \\
\hline Noch in Ausbildung & $18,3 \%$ & 23 & $25,9 \%$ & 21 & $\mathrm{chi}^{2}=1,734$ & $\mathrm{df}=1$ & $p=0,188$ \\
\hline $\begin{array}{l}\text { Abgeschlossene } \\
\text { Ausbildung }\end{array}$ & $36,5 \%$ & 46 & $24,7 \%$ & 20 & $\mathrm{chi}^{2}=3,170$ & $d f=1$ & $\mathrm{p}=0,075$ \\
\hline $\begin{array}{l}\text { Abgeschlossenes } \\
\text { Studium }\end{array}$ & $5,6 \%$ & 7 & $3,7 \%$ & 3 & \multicolumn{3}{|c|}{ nicht auswertbar } \\
\hline $\begin{array}{l}\text { Verwandte mit } \\
\text { psychiatrischen } \\
\text { Diagnosen }\end{array}$ & \multicolumn{2}{|c|}{$n=92$} & \multicolumn{2}{|c|}{$n=85$} & & & \\
\hline $\mathrm{Ja}$ & $77,2 \%$ & 71 & $38,8 \%$ & 33 & \multirow{2}{*}{$\operatorname{chi}^{2}=26,814$} & \multirow{2}{*}{$d f=1$} & \\
\hline Nein & $22,8 \%$ & 21 & $61,2 \%$ & 52 & & & $p<0.001$ \\
\hline $\begin{array}{l}\text { Verwandte mit } \\
\text { Hinweisen auf eine } \\
\text { BPS }\end{array}$ & $n=$ & & $\mathrm{n}=$ & 85 & & & \\
\hline $\mathrm{Ja}$ & $86,2 \%$ & 50 & $42,4 \%$ & 36 & & & \\
\hline Nein & $13,8 \%$ & 8 & $57,6 \%$ & 49 & $\mathrm{chi}^{2}=27,65$ & $d f=1$ & $p<0.001$ \\
\hline
\end{tabular}


Eine Gegenüberstellung der beiden Gruppen bezüglich der Soziodemographie zeigte, dass heute weniger Patienten verheiratet sind $\left(\mathrm{ch}^{2}=8,527 ; \mathrm{p}=0,003\right)$, signifikant weniger arbeitslos sind $\left(\mathrm{chi}^{2}=3,518 ; \mathrm{p}=0,061\right)$; die meisten leben ohne Partner $\left(c^{2} i^{2}=22,153 ; p<0.001\right)$. Die Tabelle zeigt auf, dass einerseits mehr Patienten mit einer Ausbildung ( $\left.\mathrm{chi}^{2}=2,793 ; \mathrm{p}=0,095\right)$ angefangen haben, jedoch weniger diese abgeschlossen haben, verglichen mit den Jahren 1996-2004 (chi² $=3,170 ; p=0,075$ ). Es sind signifikant mehr Hauptschüler $\left(c^{2} i^{2}=4,891 ; p=0,027\right)$ dafür wesentlich weniger Abiturienten ( $\left.c \mathrm{i}^{2}=5,977 ; p=0,014\right)$. In der zweiter Gruppe waren signifikant weniger Verwandte mit psychiatrischen Diagnosen $\left(c^{2}{ }^{2}=26,814, p<0,0019\right)$, als in den Jahren davor.

Zusammengefasst zeigt die Gegenüberstellung in Bezug auf die Sozialdemographie, dass unter den Patienten mit BPS in dem Untersuchungsraum 2008-2012

- weniger verheiratet waren

- mehr ohne Partner lebten

- mehr Zuhause lebten

- weniger arbeitslos waren

- mehr eine Ausbildung begonnen haben, aber weniger diese erfolgreich beendeten

- mehr die Hauptschule besuchten und weniger das Abitur abgelegten und

- weniger in der Anamnese angaben, Verwandte 1. Grades mit psychiatrischer Diagnose zu haben. 


\section{Abbildung 19 Vergleich Ehe prozentual}

rot: Patienten in den Jahren 1996-2004 ( $\mathrm{N}=139)$

blau: Patienten in den Jahren 2008-2012 (N=87)

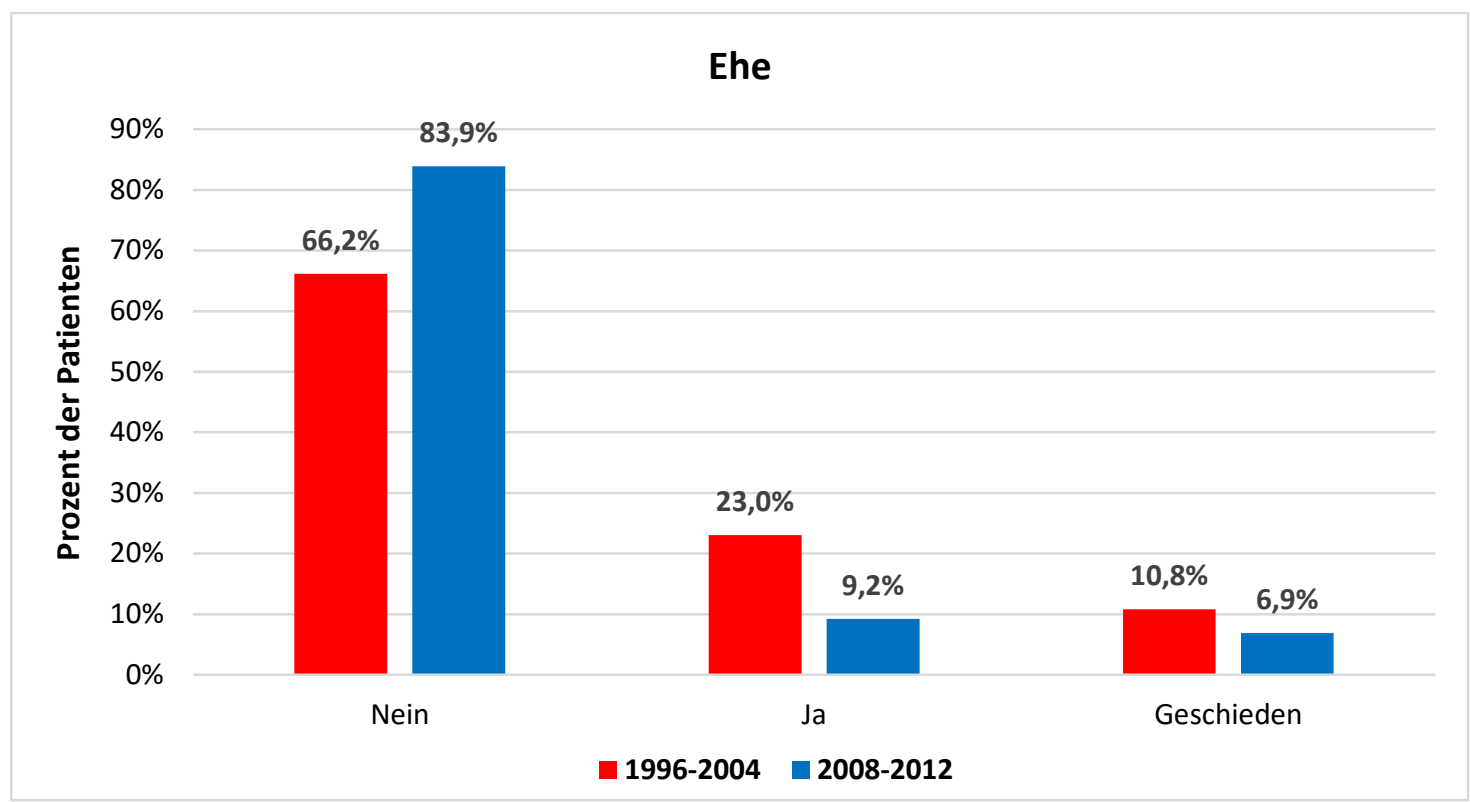

\section{Abbildung 20 Vergleich Ehe statistisch}

rot: Patienten in den Jahren 1996-2004 ( $\mathrm{N}=139)$

blau: Patienten in den Jahren 2008-2012 (N=87)

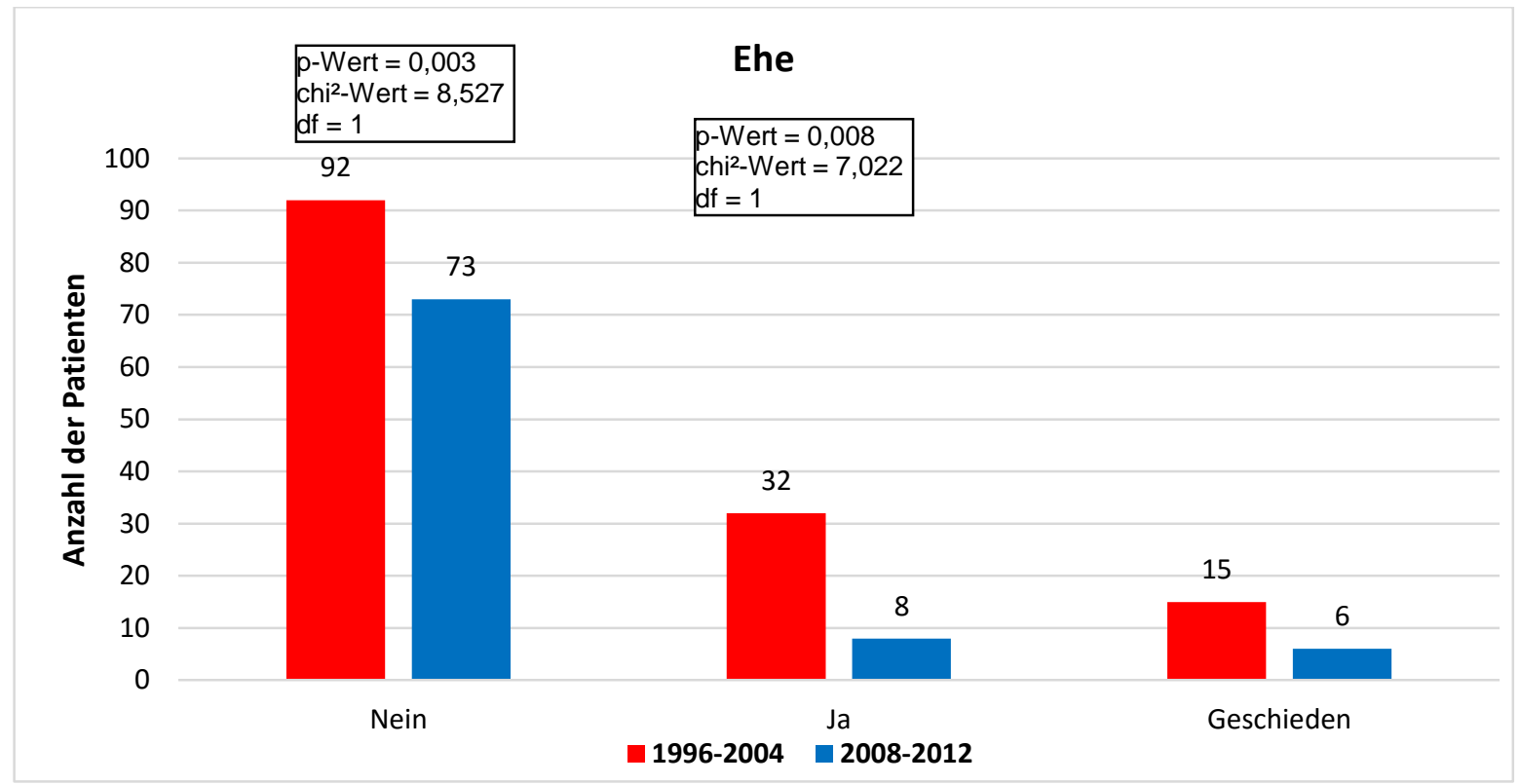

Die Abbildung 20 zeigt, dass in dem Zeitintervall 2008-2012 signifikant weniger Patienten verheiratet waren als in den Jahren 1996-2004. 


\section{Abbildung 21 Vergleich Partnerschaft prozentual}

rot: Patienten in den Jahren 1996-2004 ( $\mathrm{N}=139)$

blau: Patienten in den Jahren 2008-2012 (N=87)



\section{Abbildung 22 Vergleich Partnerschaft statistisch}

rot: Patienten in den Jahren 1996-2004 ( $\mathrm{N}=139)$

blau: Patienten in den Jahren 2008-2012 (N=87)

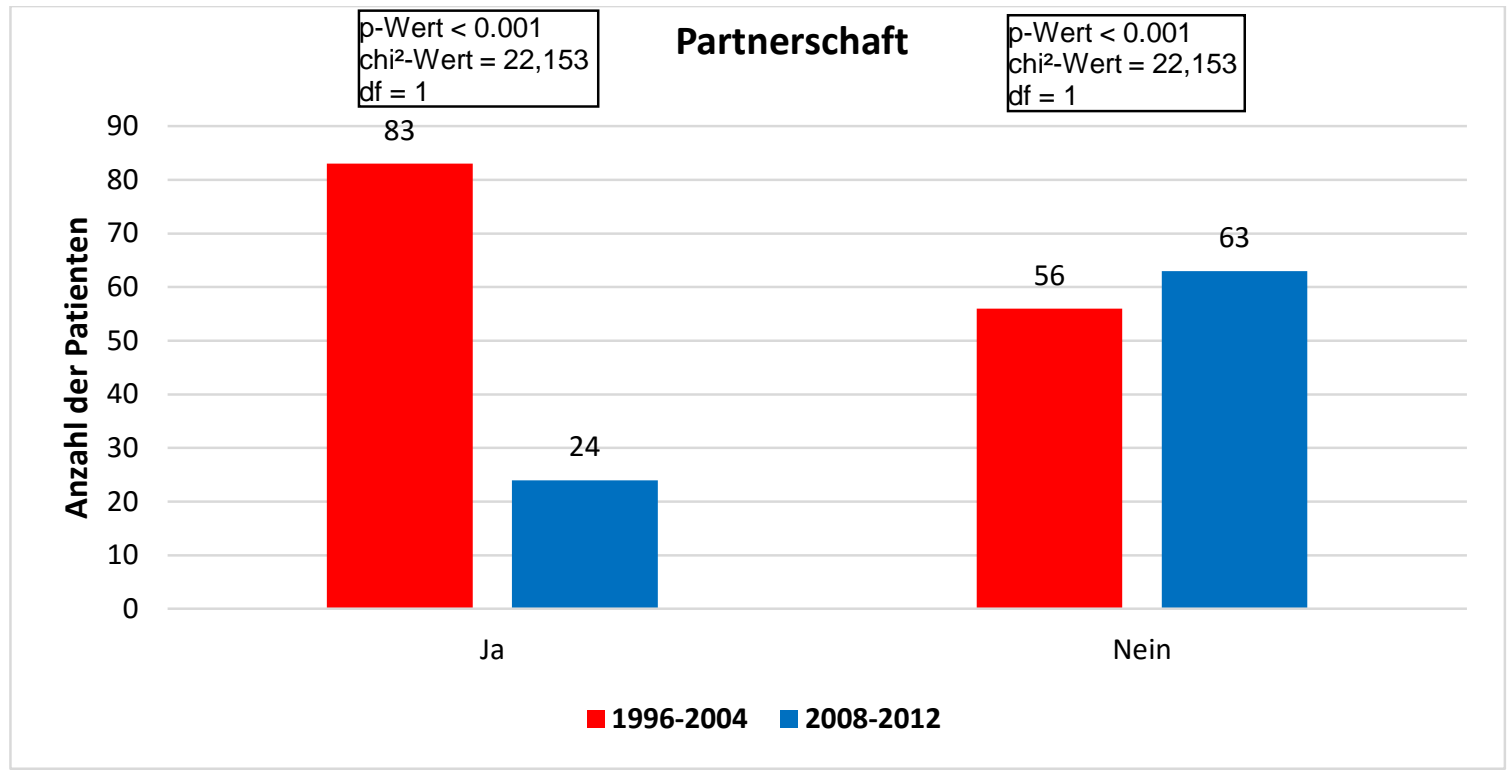

Vergleicht man beide Gruppen bezüglich der Partnerschaft, ergeben sich erhebliche

Unterschiede zwischen den beiden Gruppen. In den aktuellen Untersuchungen (2008-

2012) leben deutlich wenige Patienten in fester Partnerschaft $\left(p<0.001\right.$; $\operatorname{chi}^{2}=22,153$,

$d f=1)$ als in Jahren davor $\left(p<0.001 ; c^{2}=22,153 ; d f=1\right)$. 
Abbildung 23 Vergleich Partnerwechsel prozentual

rot: Patienten in den Jahren 1996-2004 ( $\mathrm{N}=131)$

blau: Patienten in den Jahren 2008-2012 (N=70)

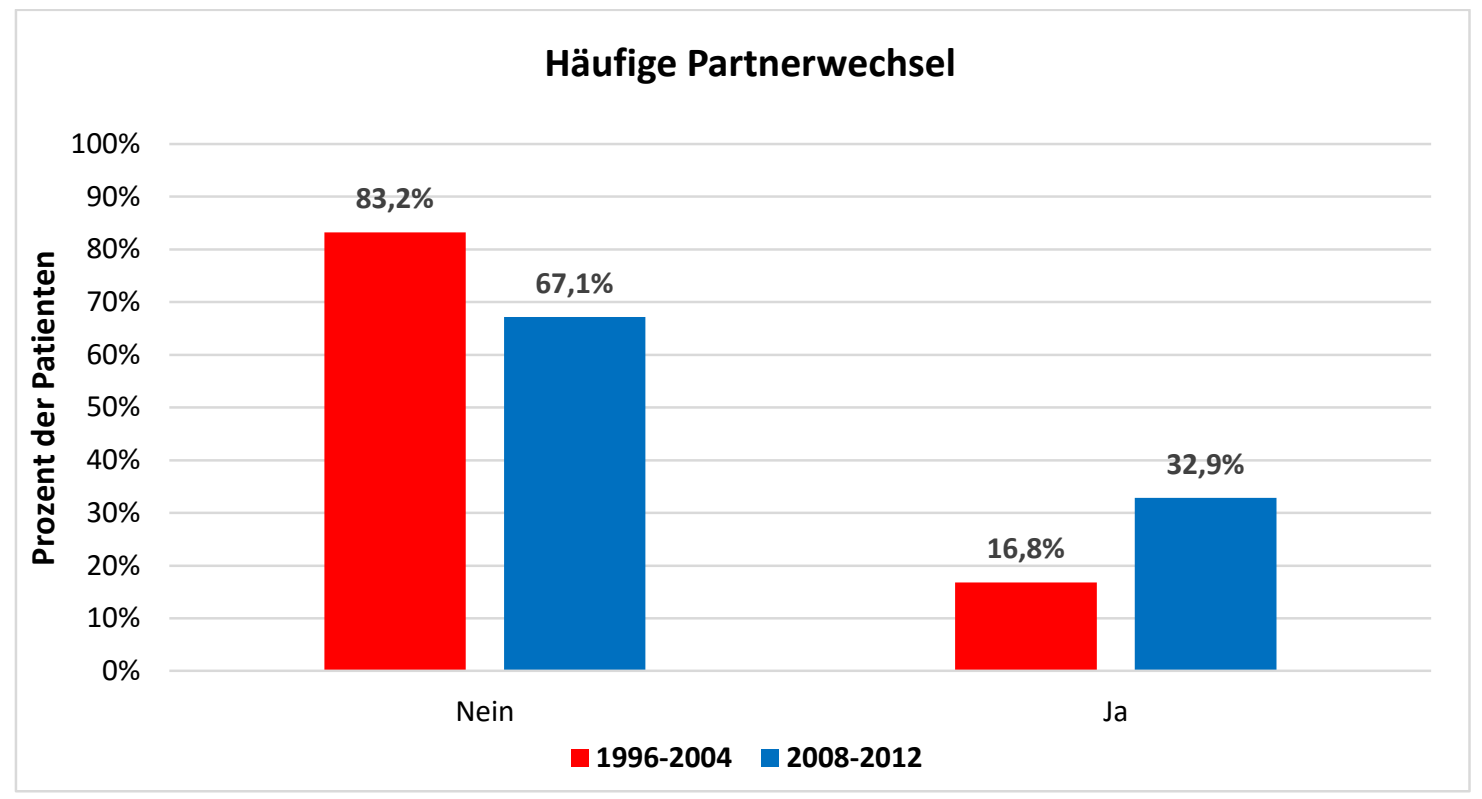

Abbildung 24 Vergleich Partnerwechsel statistisch

rot: Patienten in den Jahren 1996-2004 ( $\mathrm{N}=131)$

blau: Patienten in den Jahren 2008-2012 (N=70)

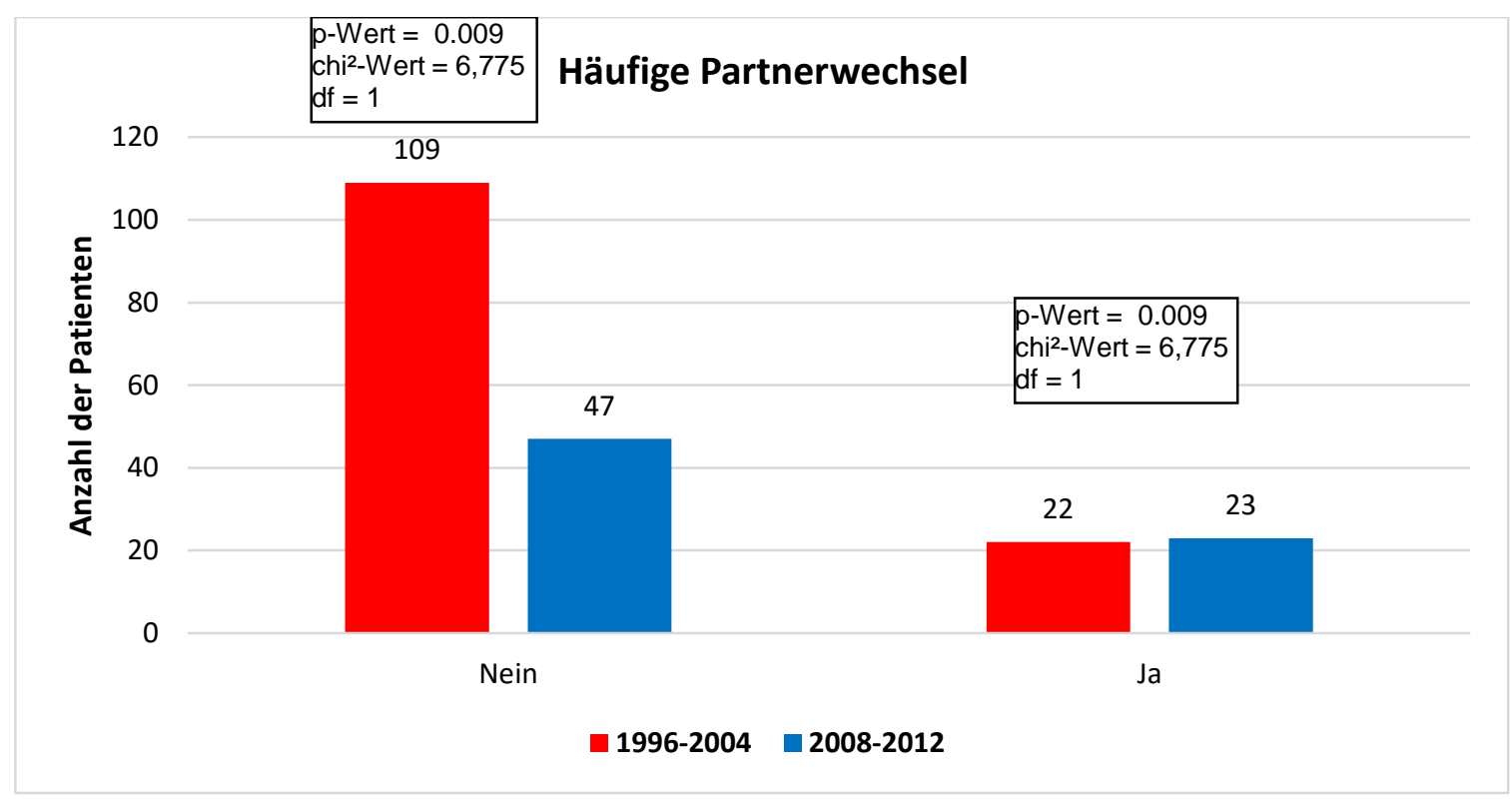

In Bezug auf die Häufigkeit von Partnerwechsel wurden zwei Signifikanztestungen durchgeführt. Dabei zeigte sich, dass bei Patienten in den Jahren 2008-2012 häufiger Partnerwechsel dokumentiert wurden als in den Jahren 1996-2004 ( $p$-Wert =0,009; $\left.\operatorname{chi}^{2}=6,775 ; \mathrm{df}=1\right)$. 


\section{Abbildung 25 Vergleich sexuelle Ausrichtung prozentual}

rot: Patienten in den Jahren 1996-2004 (N=126)

blau: Patienten in den Jahren 2008-2012 (N=87)

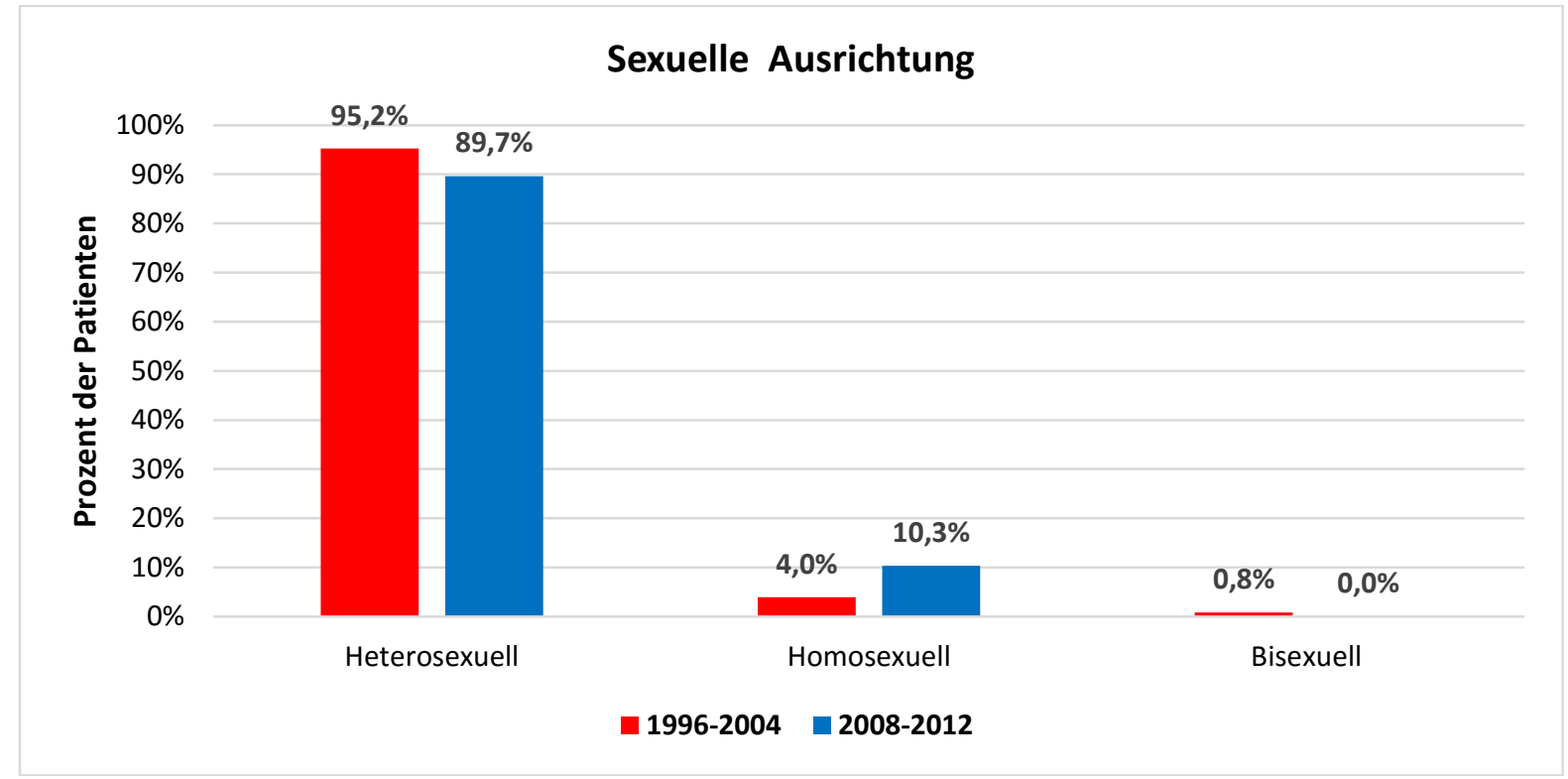

Abbildung 26 Vergleich sexuelle Ausrichtung statistisch

rot: Patienten in den Jahren 1996-2004 ( $\mathrm{N}=126)$

blau: Patienten in den Jahren 2008-2012 (N=87)

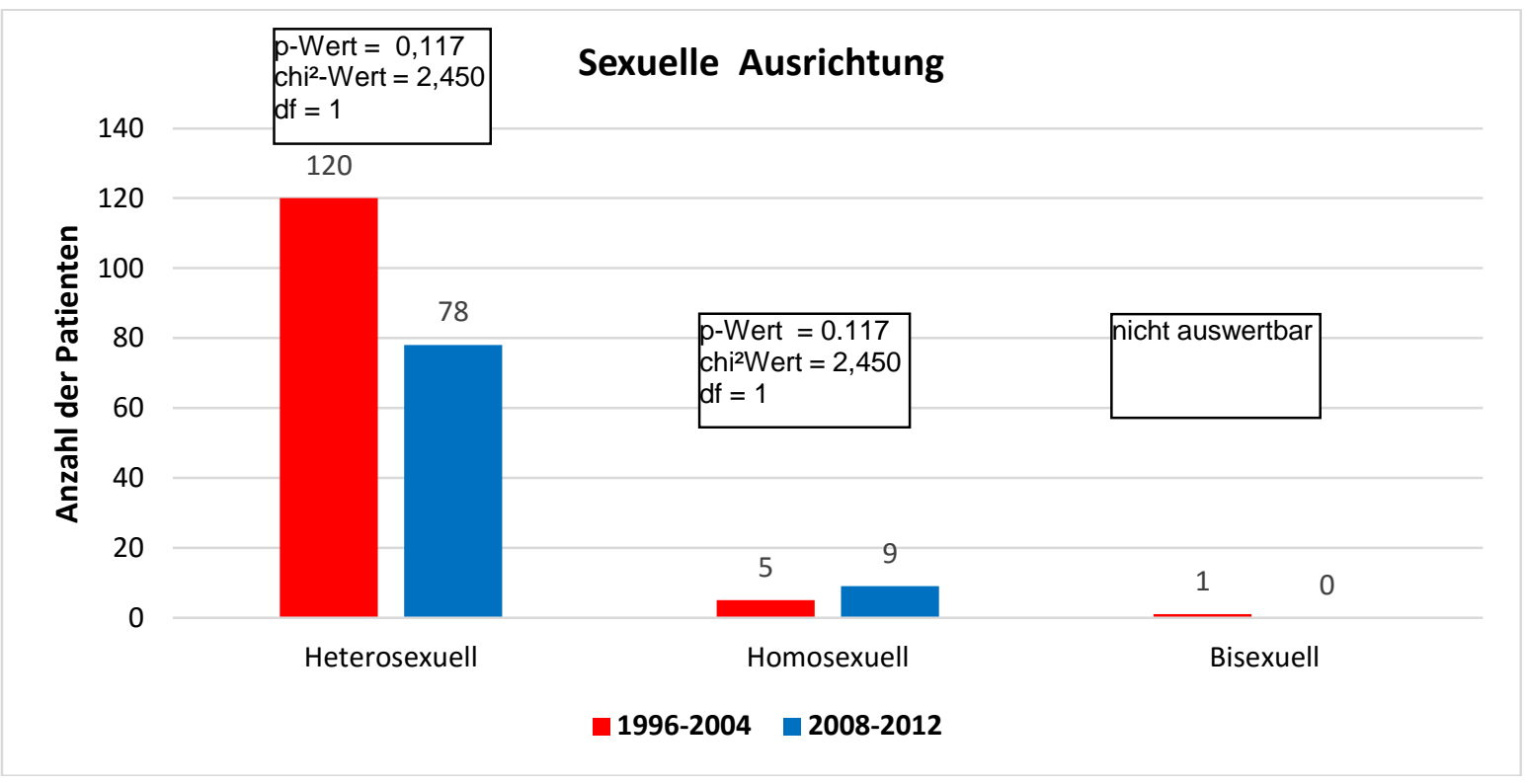

Eine Gegenüberstellung der sexuellen Ausrichtung zeigte keine signifikanten Unterschiede zwischen den beiden untersuchten Gruppen. Nominell finden sich mehr homosexuelle Patienten, der überwiegende Anteil war heterosexuell. 
Abbildung 27 Vergleich Wohnsituation prozentual

rot: Patienten in den Jahren 1996-2004 ( $\mathrm{N}=141)$

blau: Patienten in den Jahren 2008-2012 (N=87)

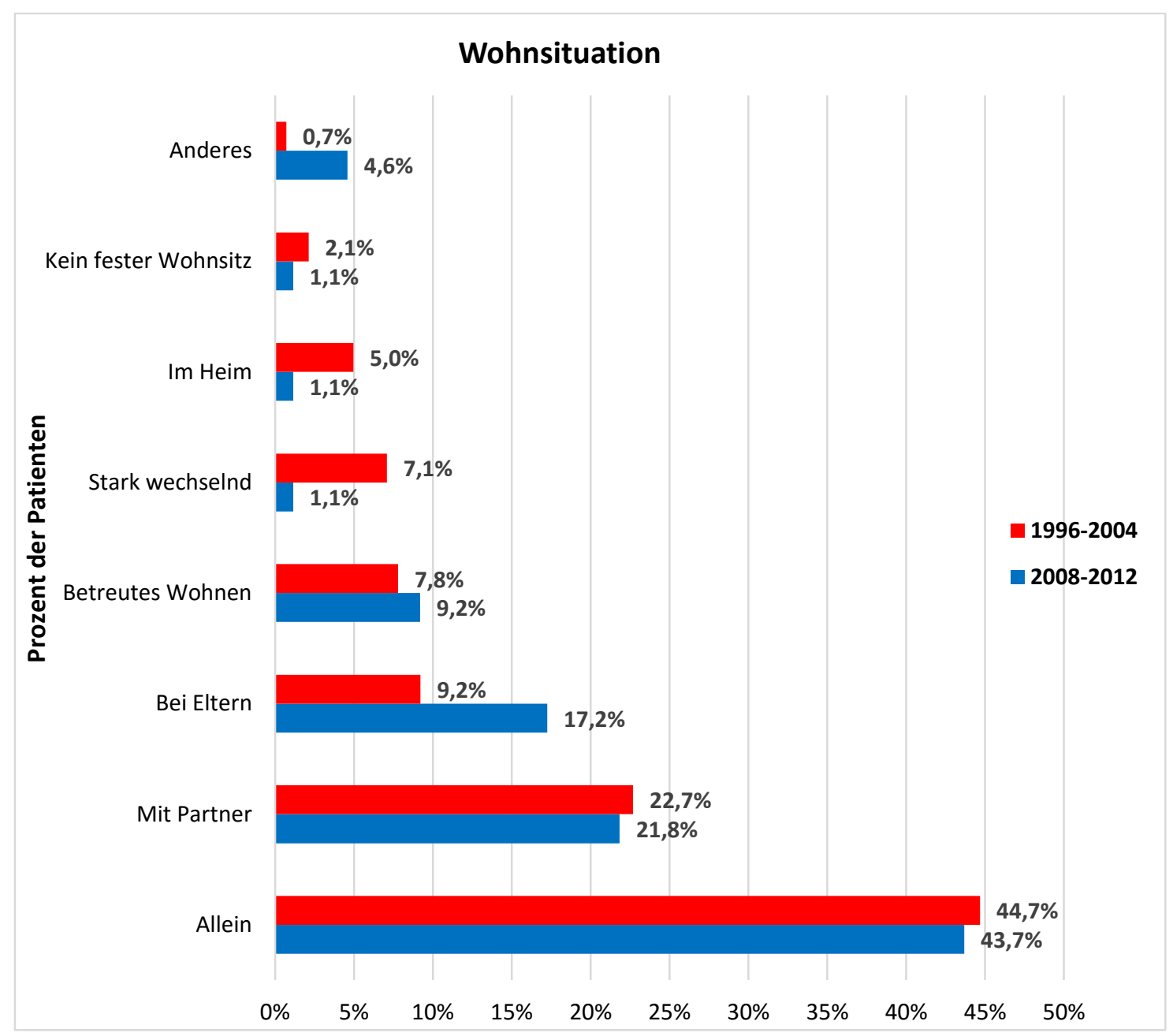




\section{Abbildung 28 Vergleich Wohnsituation statistisch}

rot: Patienten in den Jahren 1996-2004 ( $\mathrm{N}=141)$

blau: Patienten in den Jahren 2008-2012 (N=87)

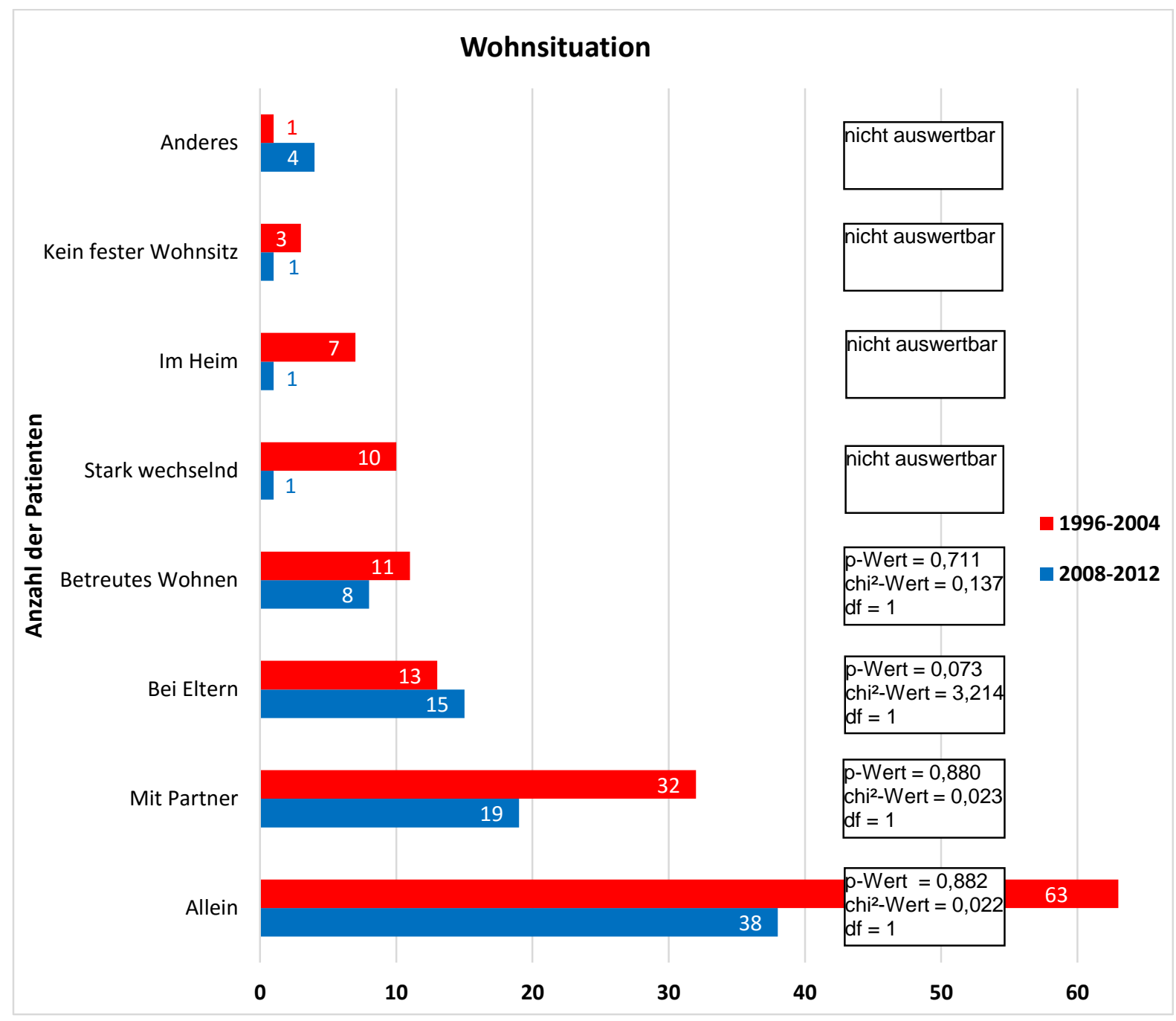

Wie auch oben dargestellten Abbildungen illustrieren, ergaben sich eher wenige Unterschiede zwischen den beiden Gruppen. Es zeigte sich kein signifikanter Effekt, jedoch ein Trend dazu, das die Patienten aktuell häufiger bei den Eltern leben als 1996-2004. 
Abbildung 29 Vergleich Tätigkeit prozentual

rot: Patienten in den Jahren 1996-2004 ( $\mathrm{N}=138)$

blau: Patienten in den Jahren 2008-2012 (N=85)

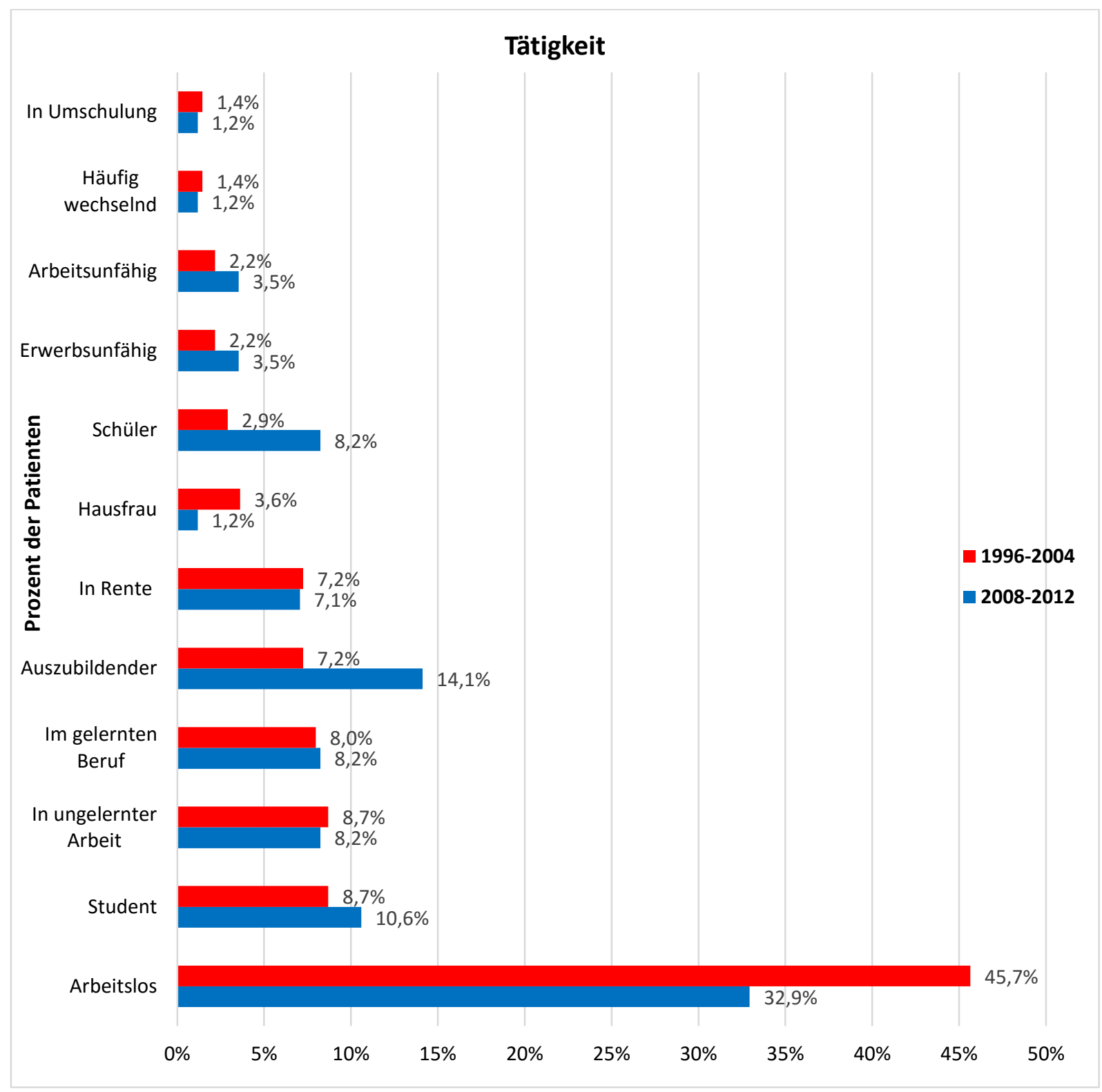




\section{Abbildung 30 Vergleich Tätigkeit statistisch}

rot: Patienten in den Jahren 1996-2004 ( $\mathrm{N}=138)$

blau: Patienten in den Jahren 2008-2012 (N=85)



Bei dem Vergleich der Tätigkeit zwischen den beiden untersuchten Gruppen ergaben sich im Wesentlichen signifikante Unterschiede nur in einzelnen Bereichen: in den

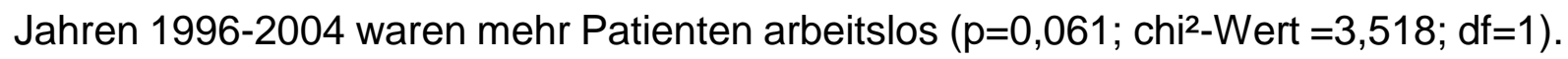
Es waren weniger Studenten $\left(p=0,638\right.$; chi $^{2}$-Wert $=0,221$; $\left.\mathrm{df}=1\right)$ und Auszubildende

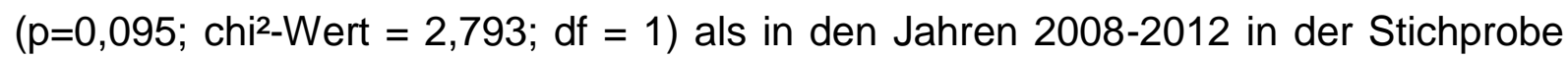
vertreten. 
Abbildung 31 Vergleich schulischer Abschluss prozentual rot: Patienten in den Jahren 1996-2004 ( $\mathrm{N}=110)$ blau: Patienten in den Jahren 2008-2012 (N=81)

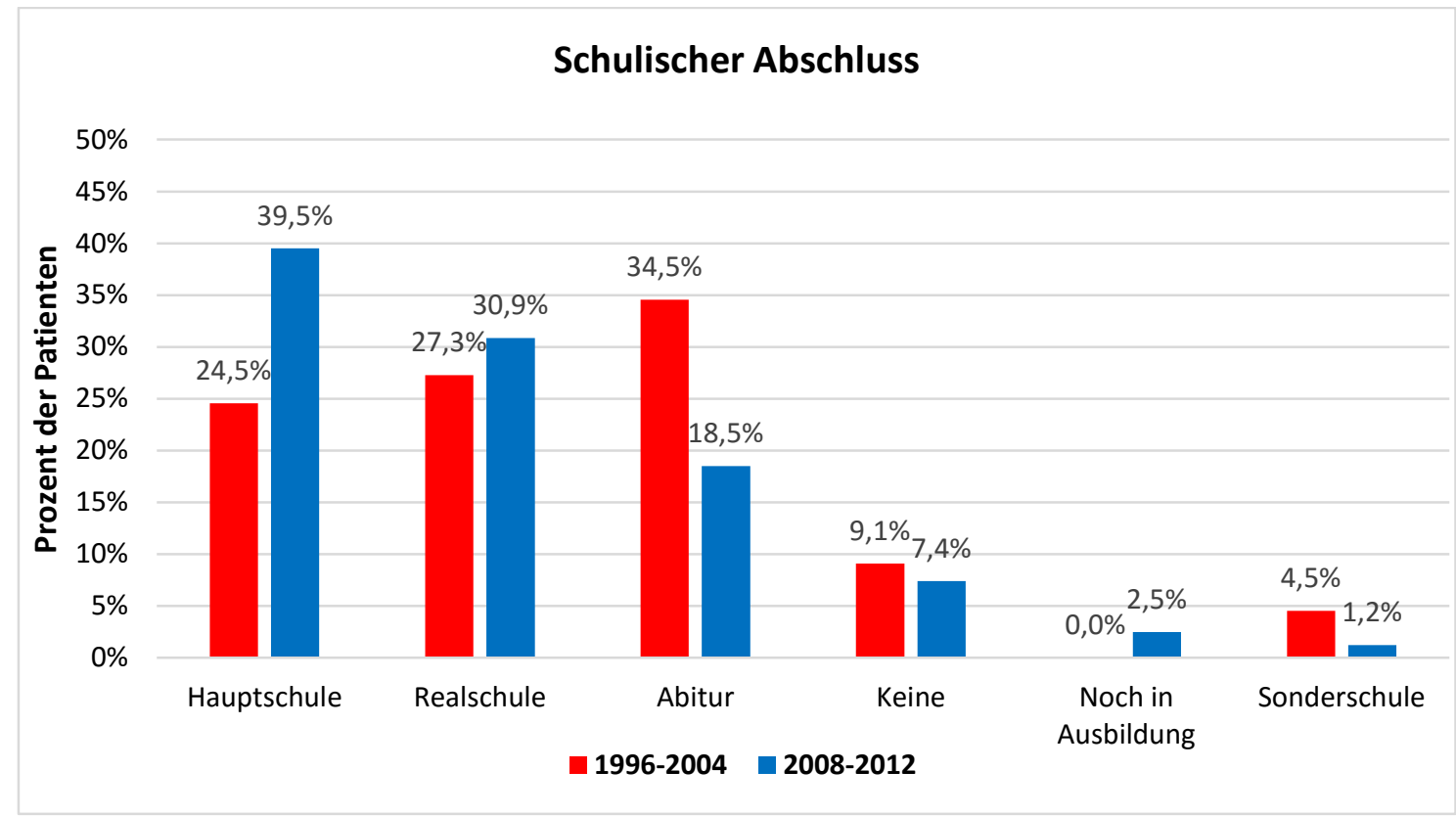

Abbildung 32 Vergleich schulischer Abschluss statistisch rot: Patienten in den Jahren 1996-2004 ( $\mathrm{N}=110)$ blau: Patienten in den Jahren 2008-2012 (N=81)

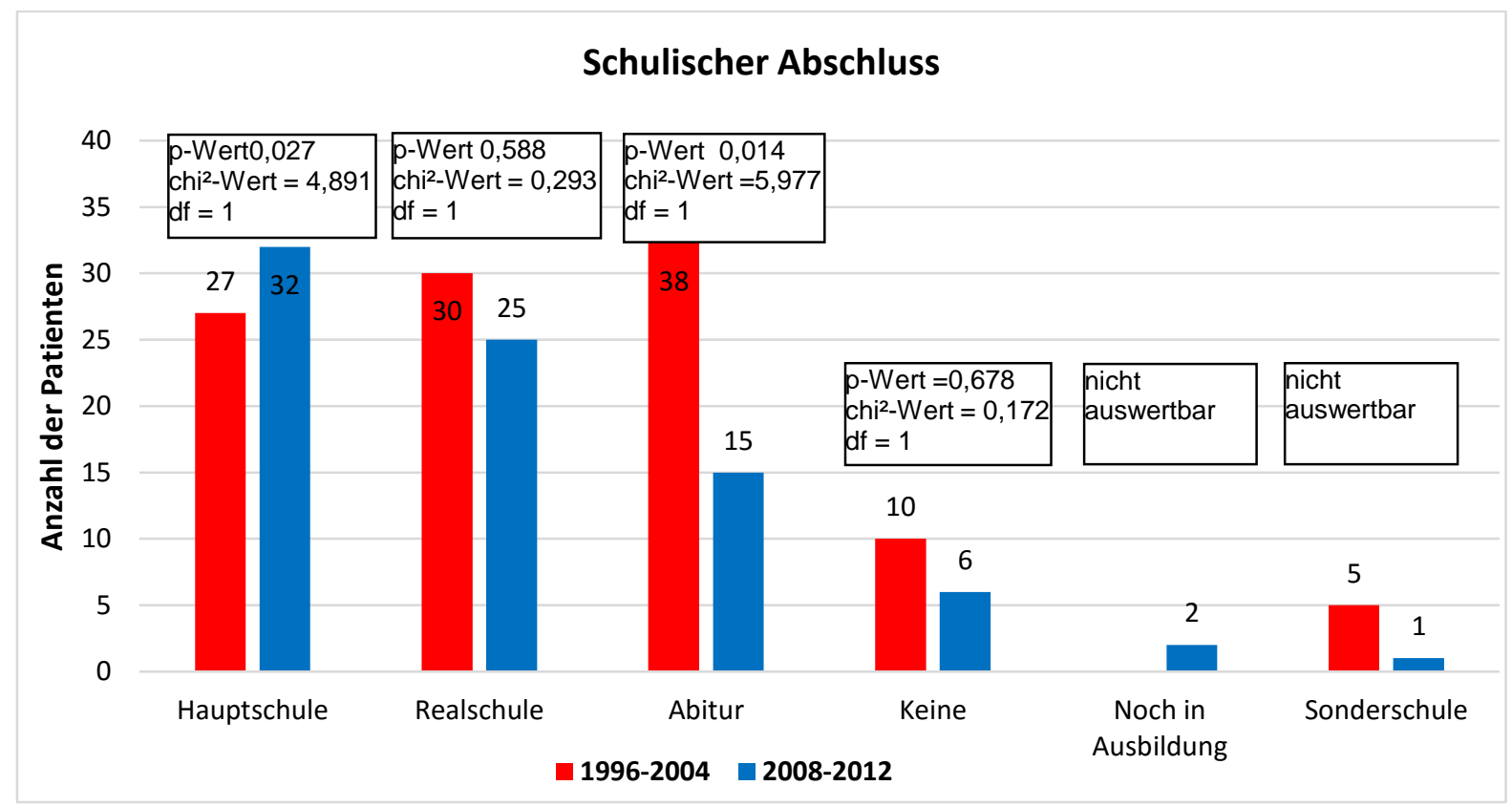

Bei der Prüfung des schulischen und beruflichen Abschlusses ergaben sich einzelne Differenzen. In den Jahren 2008-2012 hatten signifikant weniger Patienten das Abitur oder eine abgeschlossene Ausbildung. Mehr Patienten absolvierten hingegen die Haupt- oder Realschule. 
Abbildung 33 Vergleich beruflicher Abschluss prozentual rot: Patienten in den Jahren 1996-20

$04(\mathrm{~N}=126)$

blau: Patienten in den Jahren 2008-2012 (N=81)

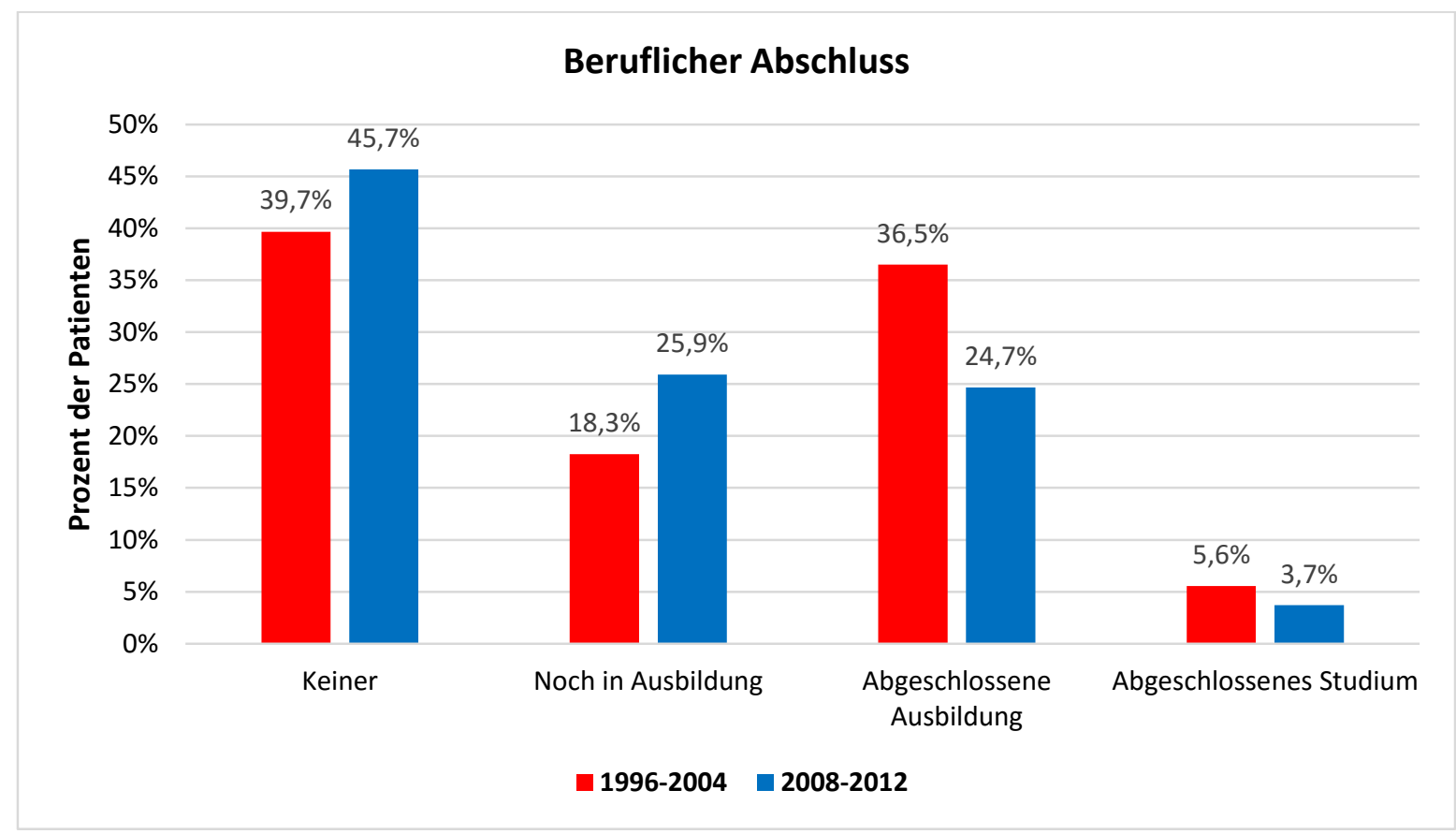

Abbildung 34 Vergleich beruflicher Abschluss statistisch rot: Patienten in den Jahren 1996-2004 ( $\mathrm{N}=126)$

blau: Patienten in den Jahren 2008-2012 (N=81)

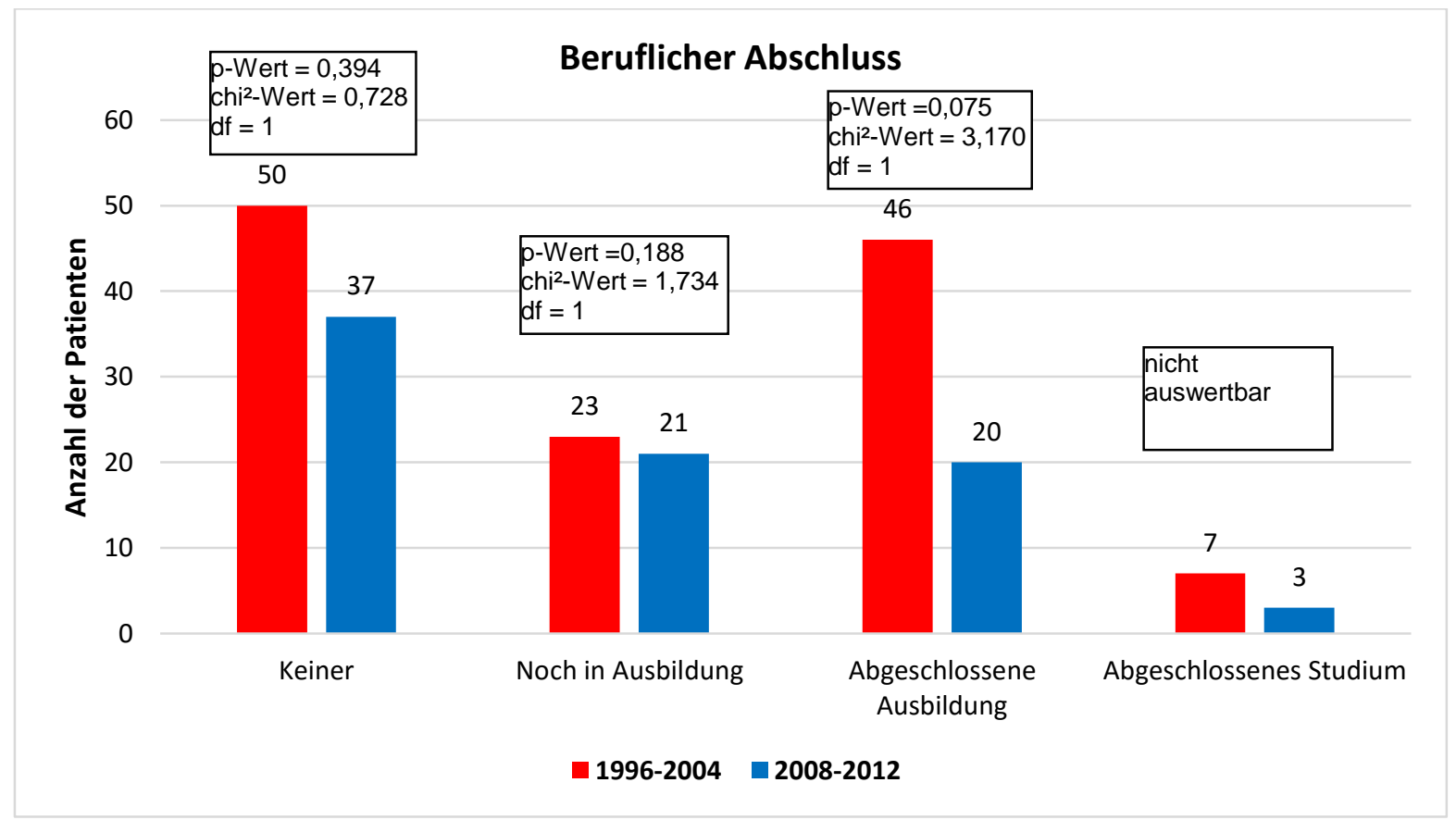


Abbildung 35 Vergleich Verwandte mit psychischen Diagnosen prozentual rot: Patienten in den Jahren 1996-2004 (N=92)

blau: Patienten in den Jahren 2008-2012 ( $\mathrm{N}=85)$

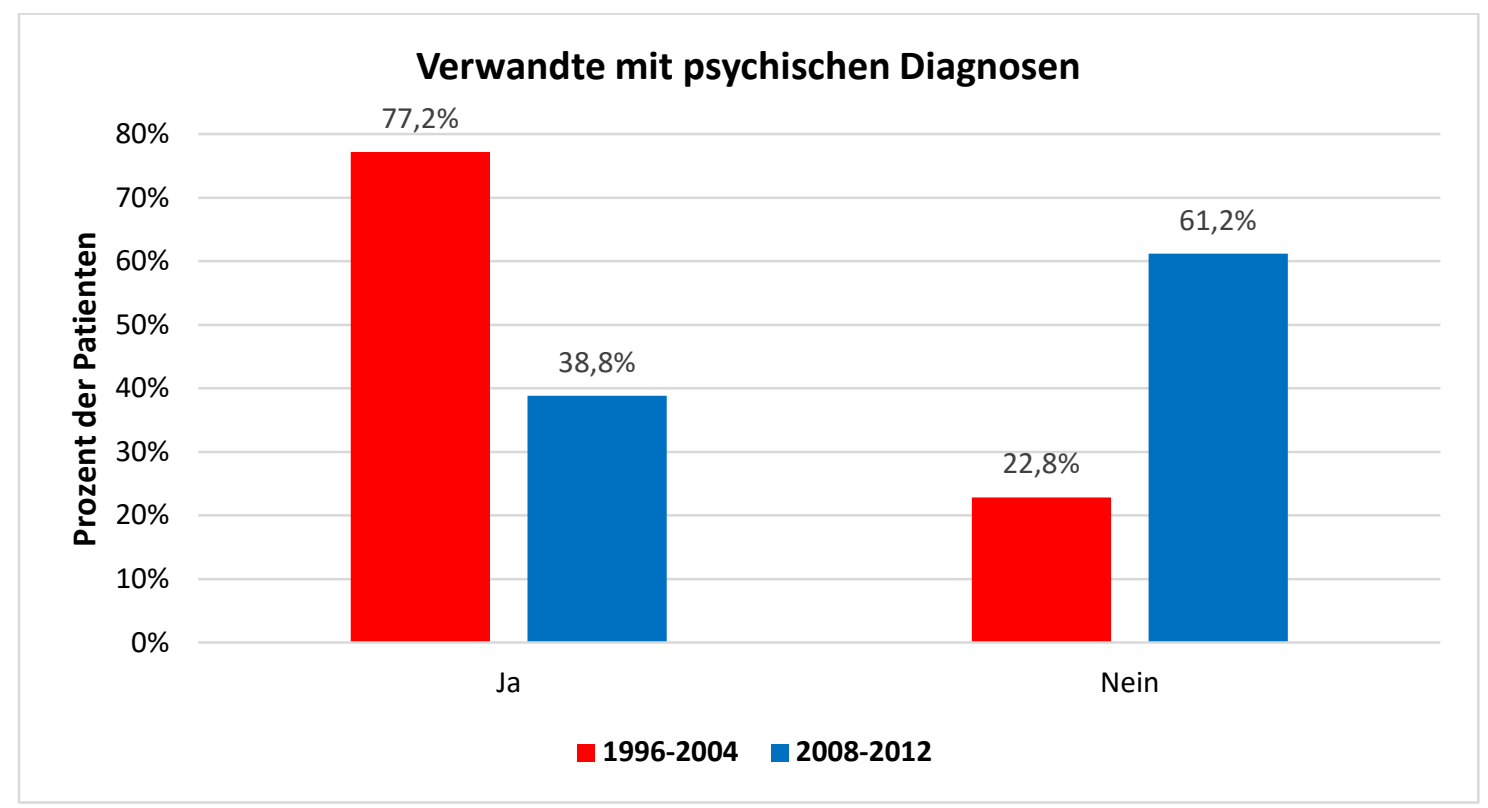

Abbildung 36 Vergleich Verwandte mit psychischen Diagnosen statistisch rot: Patienten in den Jahren 1996-2004 (N=92)

blau: Patienten in den Jahren 2008-2012 ( $\mathrm{N}=85)$

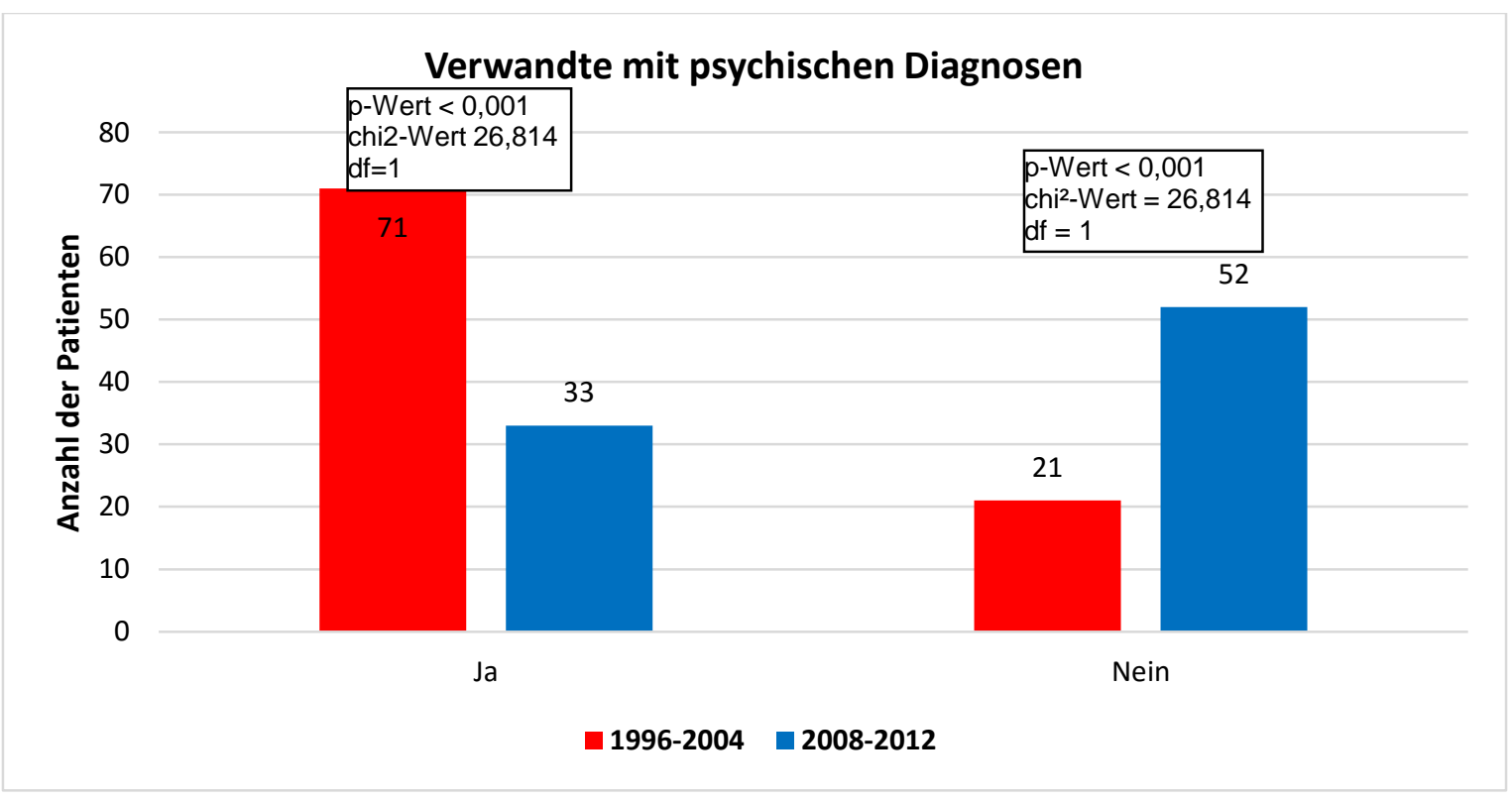

Bei der Fragestellung Verwandte 1. Grades mit psychiatrischen Diagnosen, ergab sich eine sichtbare Dominanz in dem ersten Beobachtungsraum 1996-2004. Hier wurden mit $77,2 \%$ ( $n=92)$ höhere Werte ermittelt als in dem zweiten Beobachtungsraum 20082012 mit $38,8 \%(n=85)$. 
Abbildung 37 Vergleich Verwandte mit Hinweis auf BPS prozentual rot: Patienten in den Jahren 1996-2004 (N=58)

blau: Patienten in den Jahren 2008-2012 (N=85)

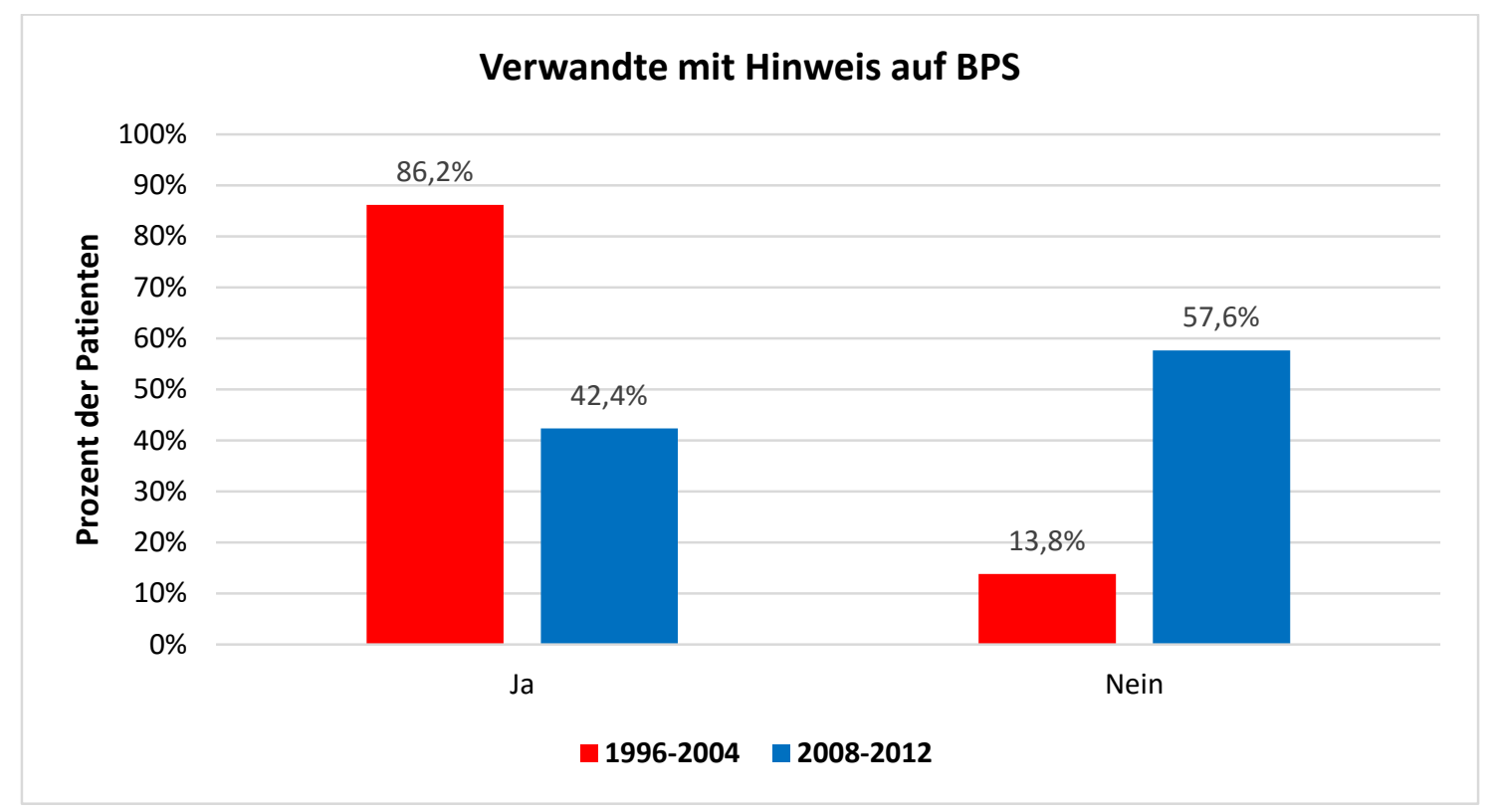

Abbildung 38 Vergleich Verwandte mit Hinweis auf BPS statistisch rot: Patienten in den Jahren 1996-2004 ( $\mathrm{N}=58)$ blau: Patienten in den Jahren 2008-2012 (N=85)

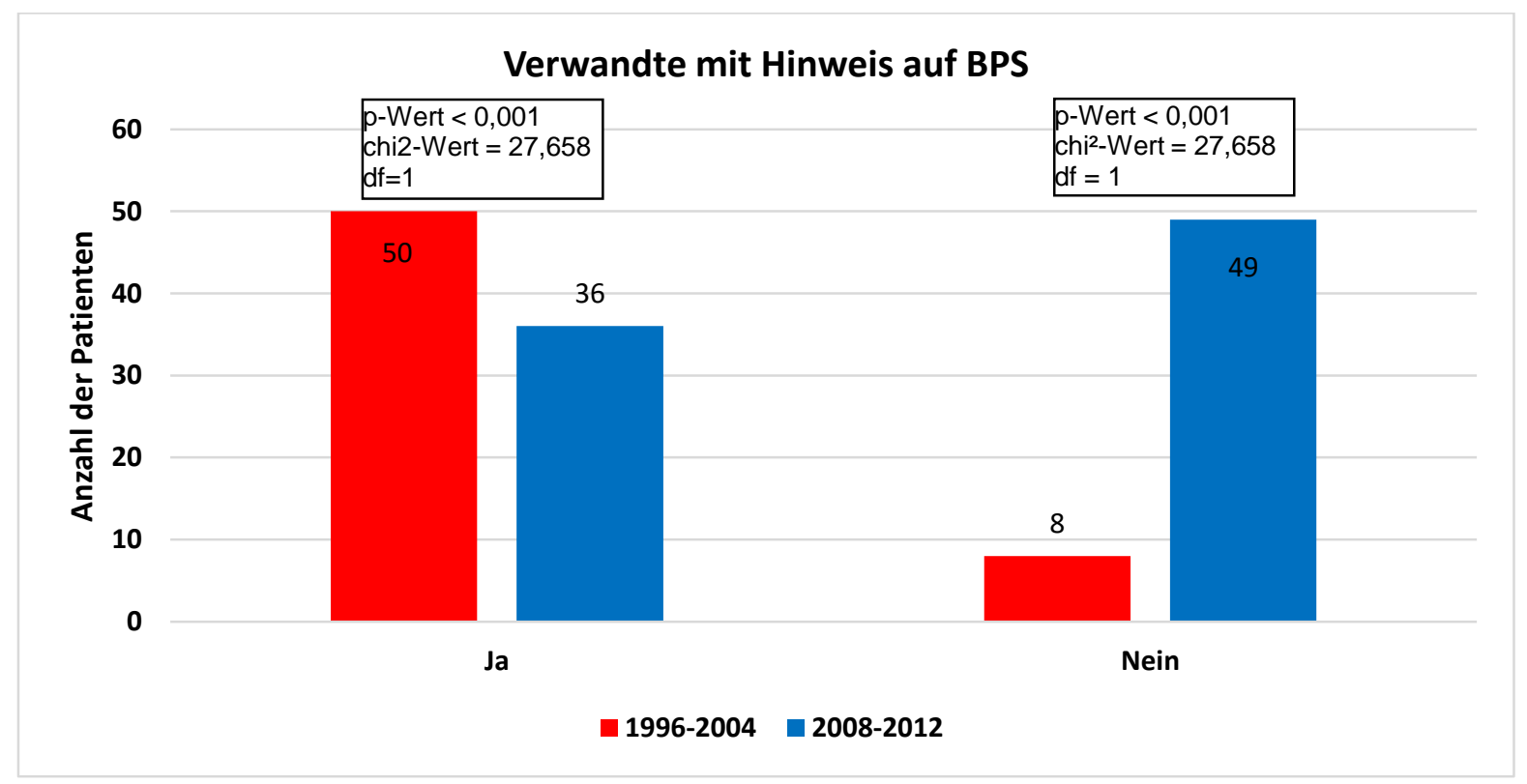

Es zeigte sich in dem Beobachtungszeitraum 2008-2012, dass weniger Patienten angaben, Verwandte mit einer BPS $z u$ haben als im davor liegenden Untersuchungszeitraum 1996-2004 (chiWert = 27,658; df=1; $p$-Wert $<0,001$ ). 


\subsubsection{Vergleich Komorbiditäten}

\section{Abbildung 39 Vergleich Komorbiditäten prozentual}

rot: Patienten in den Jahren 1996-2004 ( $\mathrm{N}=142)$

blau: Patienten in den Jahren 2008-2012 (N=86)

Legende: dunkle Balken: Störungshauptgruppen nach ICD-10; helle Balken:

Störungsuntergruppen nach ICD-10

Psychische und Verhaltensstörungen d. psychotrope Substanzen, F1x

Neurotische, Belastungs- und somatoforme Störungen, $\mathrm{F} 4 \mathrm{x}$
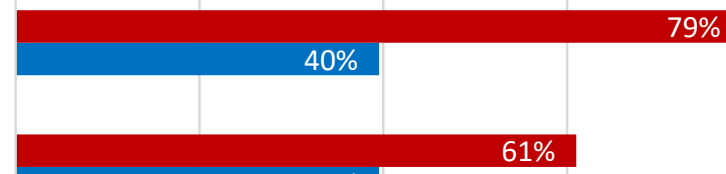

Reaktionen auf schwere Belastungen und Anpassungsstörungen, F43

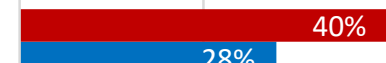

$28 \%$

Phobische, Angst- und Zwangsstörungen, F40-42

weitere Persönlichkeits-und Verhaltensstörungen, F6x

Dissoziative Störungen, F44

Somatoforme Störungen, F45

Affektive Störungen, F3x

Essstörungen, F50

Verhaltungsauffälligkeiten in Verbindung mit körperlichen Störungen und Faktoren, F5x

Verhaltens- und emotionale Störungen mit Beginn in der Kindheit und Jugend, $\mathrm{F} 9 \mathrm{x}$

Entwicklungsstörungen, F8x

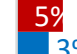

Intelligenzminderung, F7x
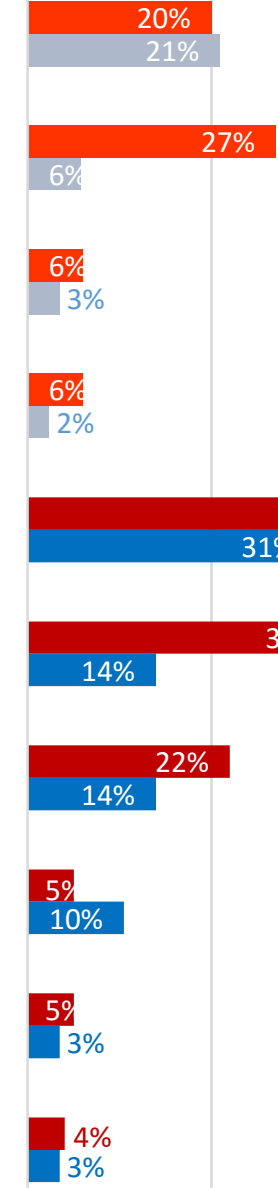

\section{$\frac{6 \%}{2 \%}$}
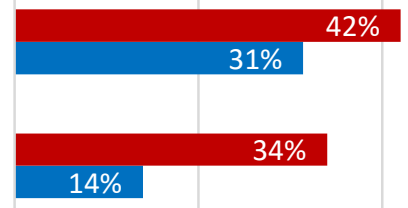

$14 \%$

Werden die Häufigkeiten der einzelnen Störungen von beiden Gruppen betrachtet, weist ein hoher Prozentsatz der Patienten zusätzliche psychische Störungen auf. Im Vordergrund stehen bei beiden Gruppen psychische- und Verhaltensstörungen durch psychotrope Substanzen, wobei aus der Abbildung 39 ersichtlich wird, dass jene im 
Zeitraum 2008-2012 nur etwa in der Hälfte der Fälle zu beobachten waren. Am zweithäufigsten waren Belastungs- oder somatoforme Störungen. Am häufigsten fanden sich hierbei posttraumatische Belastungsstörungen. Phobische Angst- und Zwangsstörungen sowie Anpassungsstörungen sind in beiden Zeitintervallen gleich geblieben.

\section{Abbildung 40 Vergleich Komorbiditäten statistisch}

rot: Patienten in den Jahren 1996-2004 ( $\mathrm{N}=142)$

blau: Patienten in den Jahren 2008-2012 (N=86)




Abbildung 40 zeigt auf, dass sich zwischen der Gruppe neurotischer Belastungs- und somatoformer Störungen, Anpassungsstörungen, Phobischer-, Angst- und Zwangsstörungen und der Gruppe affektiver Störungen keine signifikanten Unterschiede ergaben.

Ein wesentlicher Unterschied ergab sich in der Gruppe psychischer- und Verhaltungsstörungen durch psychotrope Substanzen $\left(\right.$ chi $^{2}=35,990$; $\mathrm{df}=1$; p-Wert< 0,001) sowie bei den Essstörungen (chi $=10,883$; $d f=1$; $p$-Wert < 0,001). Im zweiten Zeitintervall erfüllten nur ein Viertel der Patienten die Kriterien für eine Essstörung. Auch bei zusätzlichen Persönlichkeits- und Verhaltensstörungen waren signifikant weniger Patienten vertreten $\left(\mathrm{chi}^{2}=15,357\right.$; df $=1$; $\mathrm{p}$-Wert $\left.<0,001\right)$ als im ersten Beobachtungsintervall.

Abbildung 41 Vergleich Komorbiditäten - Psychische und Verhaltensstörungen durch psychotrope Substanzen prozentual

rot: Patienten in den Jahren 1996-2004 $(\mathrm{N}=142)$

blau: Patienten in den Jahren 2008-2012 (N=87)

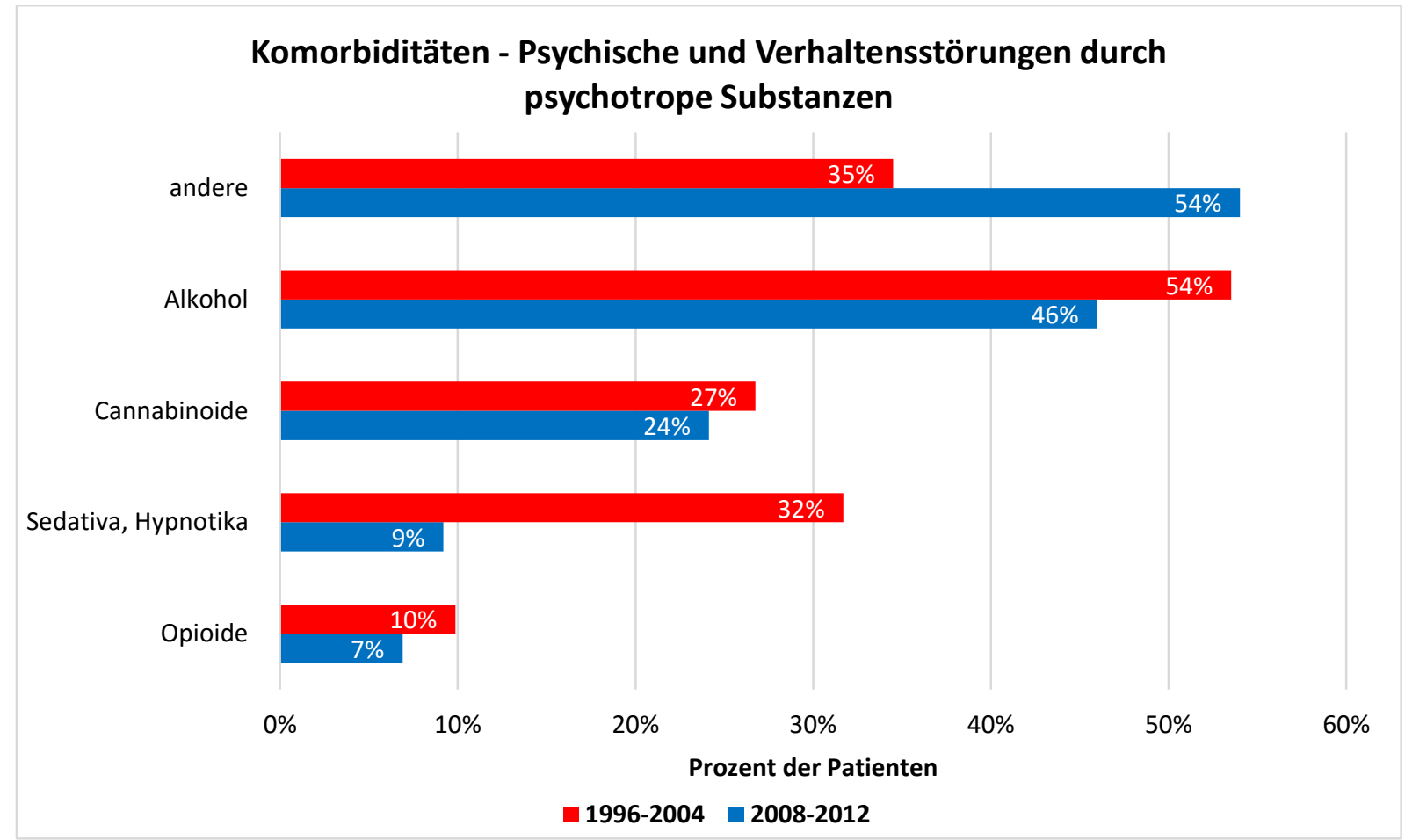




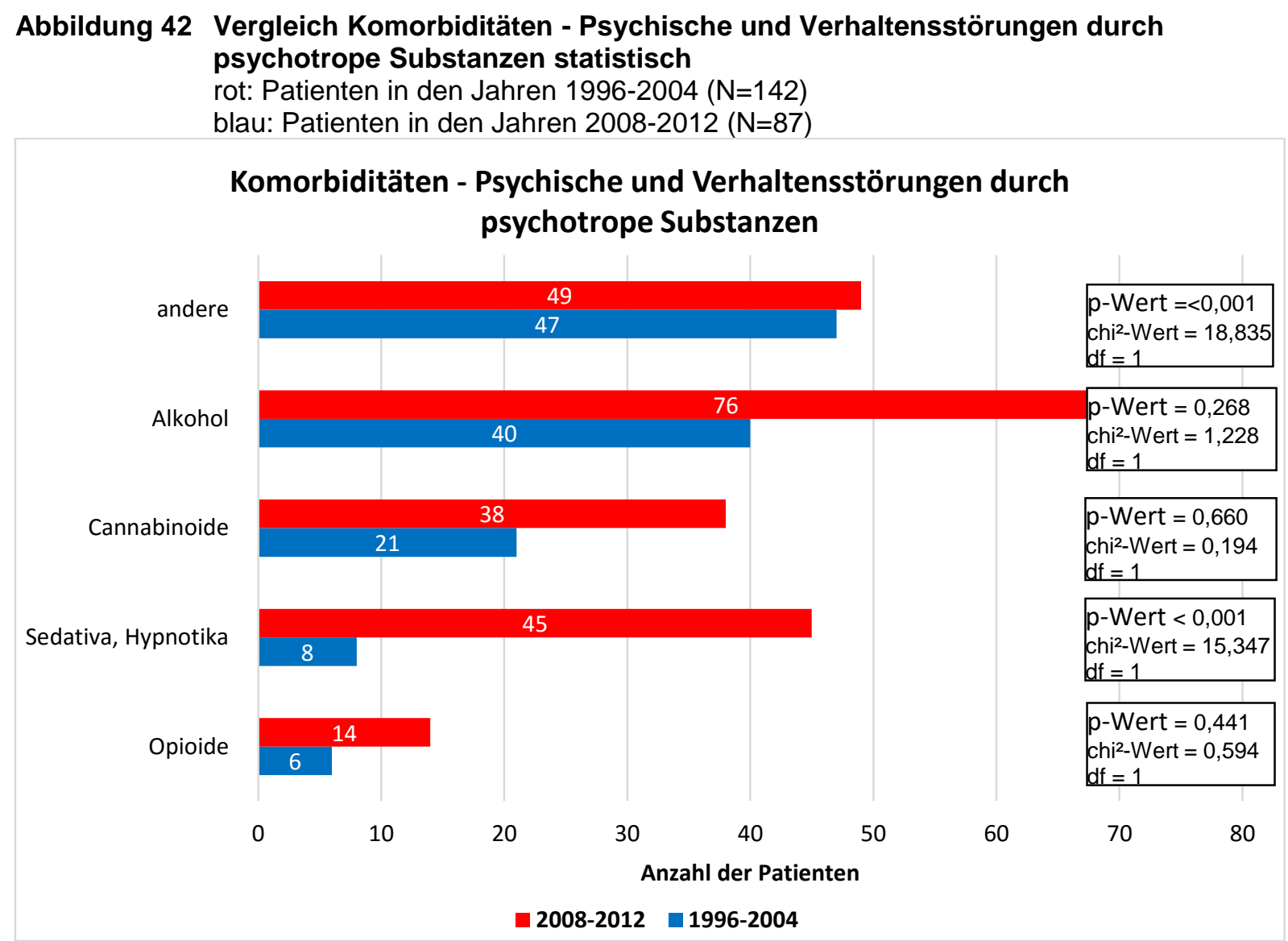

Eine Gegenüberstellung von beiden Gruppen zeigte, dass Störungen durch Alkohol, Cannabinoide und Opioide keine signifikanten Unterschiede ergaben, obwohl nominell eine Abnahme über die Zeit zu erkennen war. Störungen durch Sedativa, Hypnotika waren signifikant weniger 2008-2012 im Vergleich zu 1996-2004 vorhanden, die Restkategorie „andere Substanzen“ war signifikant höher vertreten. Es ist unklar, welche Substanzen in dieser Kategorie konkret vertreten sind, es wäre denkbar, dass moderne „Partydrogen“ wie substituierte Amphetamine, Halluzinogene und Kokain hierunter fallen. 
Abbildung 43 Vergleich Suizidversuche in der Anamnese prozentual

rot: Patienten in den Jahren 1996-2004 ( $\mathrm{N}=107)$

blau: Patienten in den Jahren 2008-2012 (N=46)

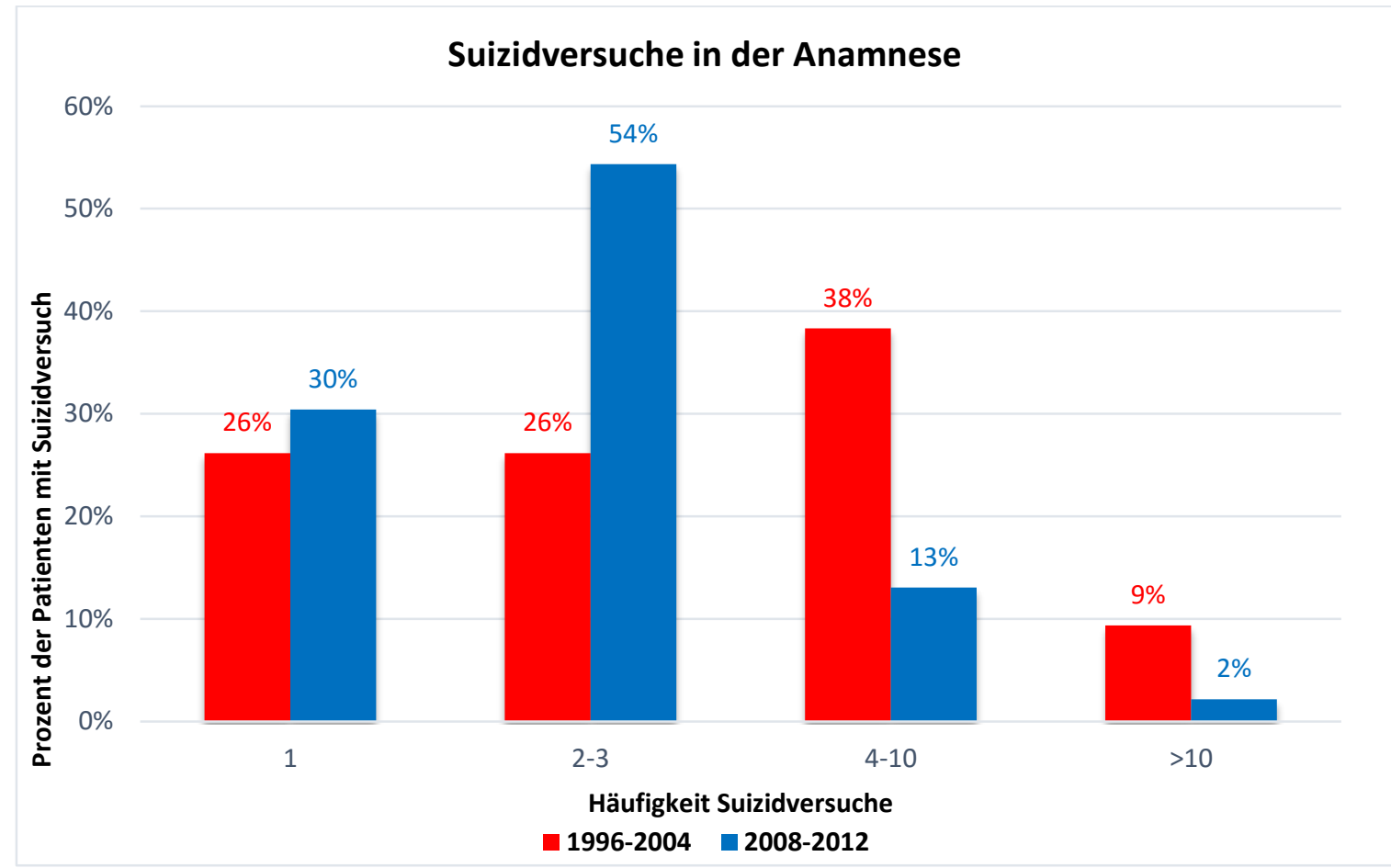

Abbildung 44 Vergleich Suizidversuche in der Anamnese statistisch rot: Patienten in den Jahren 1996-2004 ( $\mathrm{N}=107)$

blau: Patienten in den Jahren 2008-2012 (N=46)

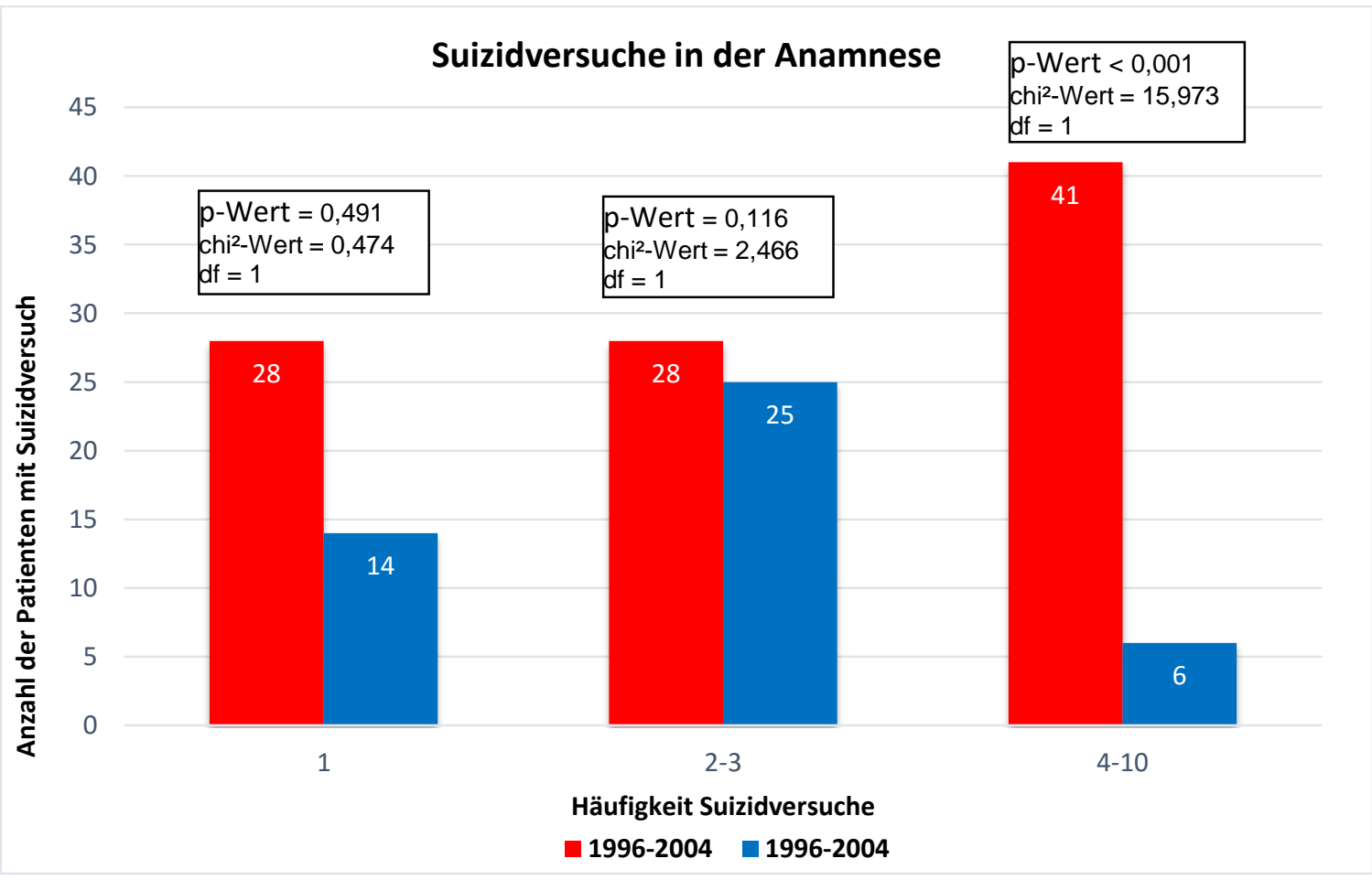


Die Abbildung 43 zeigt die prozentuale Häufigkeit der Suizidversuche in der Stichprobe. Aus der Tabelle wird sichtbar, dass es im zweiten Beobachtungsintervall in der Gruppe 2-3 Suizidversuche nominell einen Anstieg über die Zeit gab. In der der Gruppe von 4-10 Versuchen hingegen, kam es zu einer prozentualen Abnahme im Vergleich 1996-2004 zu 2008-2012.

Es ergaben sich keine signifikanten Gruppenunterschiede (Abbildung 44) hinsichtlich

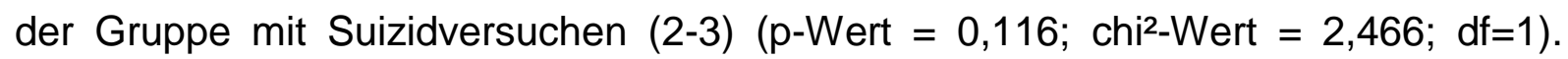
Bezüglich der Patientengruppe die 4 bis 10 Suizidversuche hatte, ergaben sich

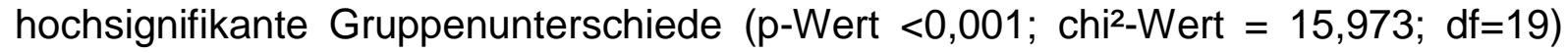
zwischen den beiden Untersuchungszeiträumen. 


\title{
3.4.3 Vergleich der Dokumentation der diagnostischen Kriterien nach ICD-10 und DSM-IV
}

\author{
Abbildung 45 Vergleich der einzelnen diagnostischen Kriterien nach ICD-10 (WHO 1993) \\ prozentual \\ rot: Patienten in den Jahren 1996-2004 ( $\mathrm{N}=142)$ \\ blau: Patienten in den Jahren 2008-2012 (N=87)
}

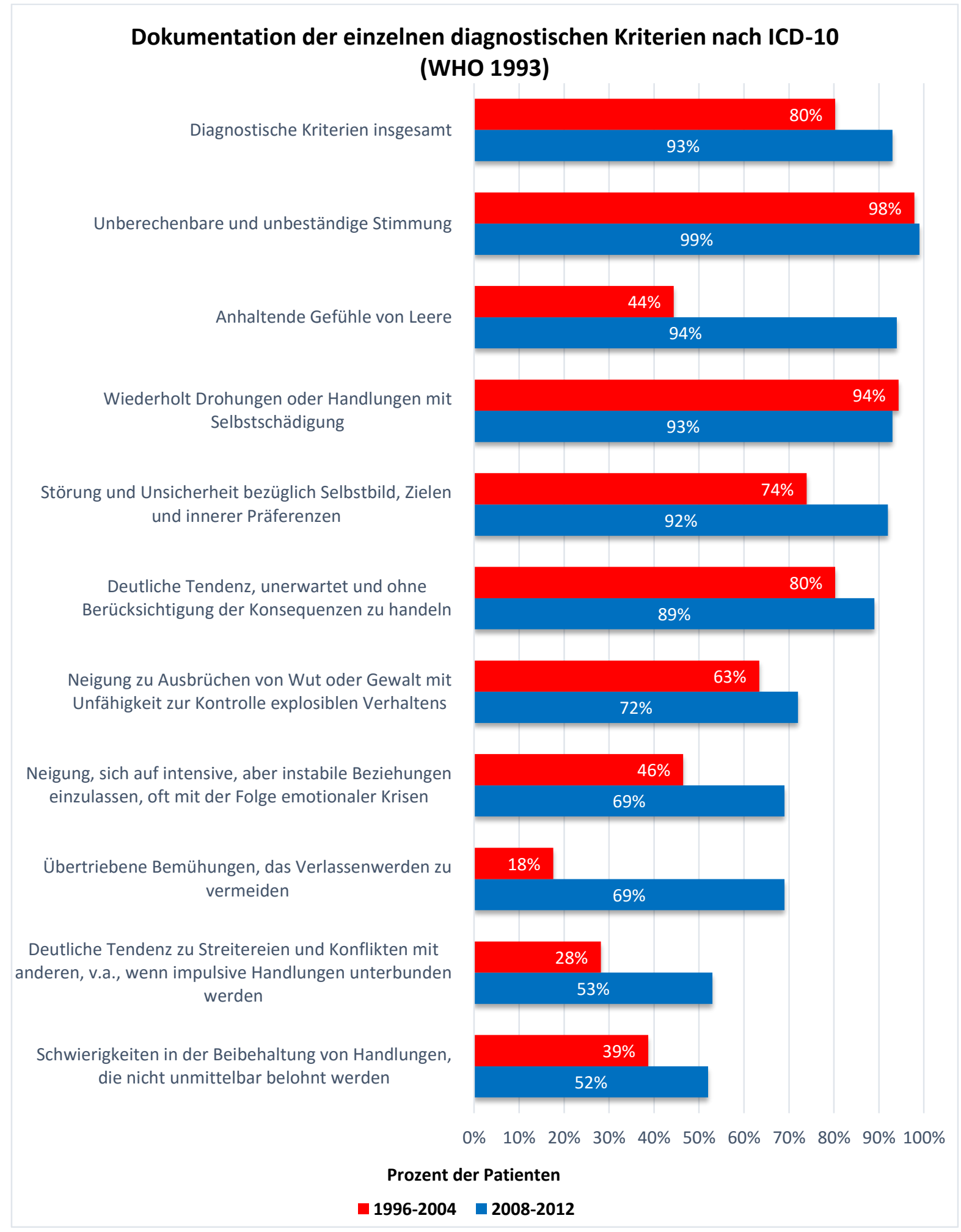




\section{Abbildung 46 Vergleich der einzelnen diagnostischen Kriterien nach ICD-10 (WHO 1993) statistisch \\ rot: Patienten in den Jahren 1996-2004 ( $\mathrm{N}=142)$ \\ blau: Patienten in den Jahren 2008-2012 (N=87)}

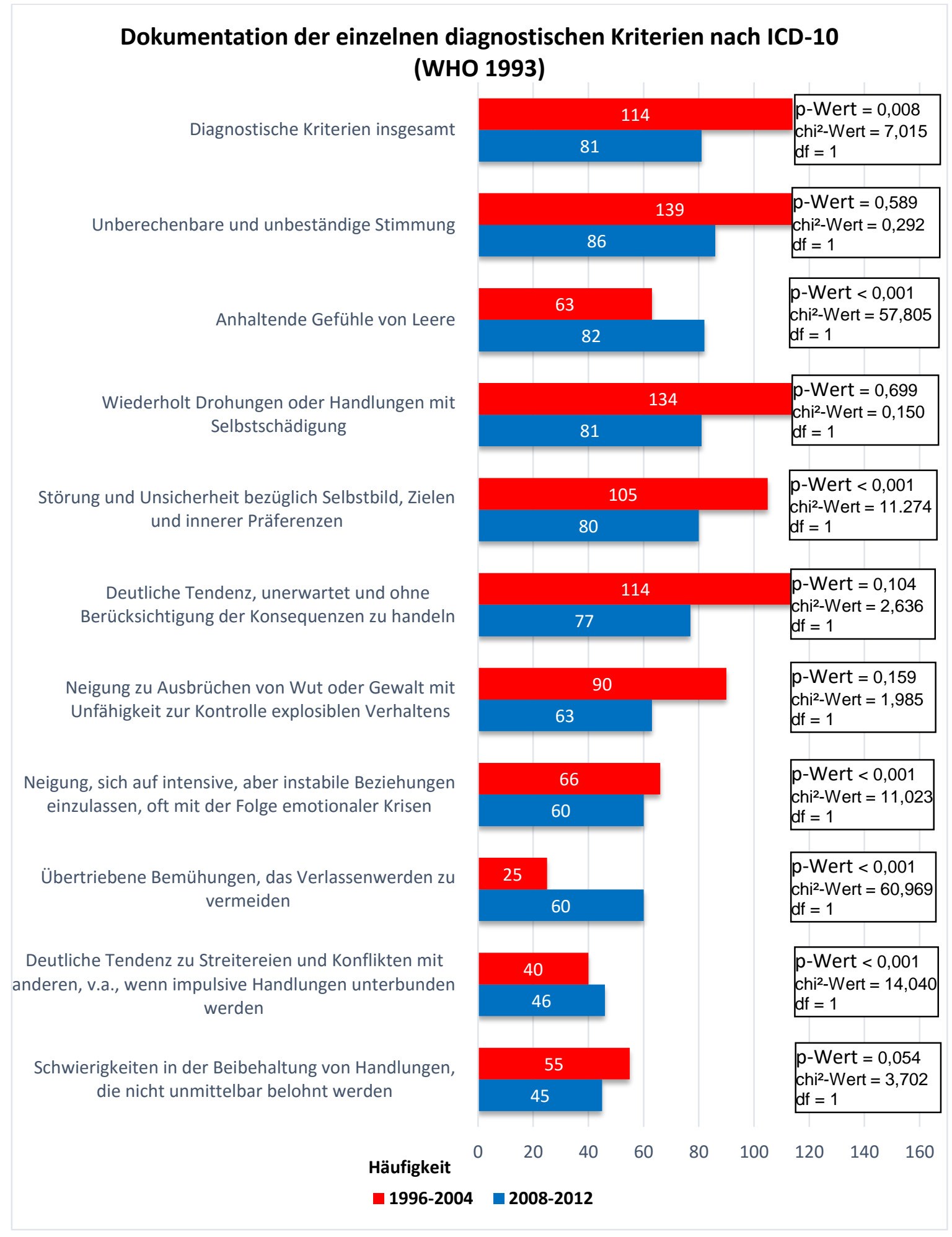

Die Häufigkeit der Diagnose konformer Beschreibung von Symptomen hat zwischen 2008-2012 deutlich zugenommen. Im Vordergrund stehen im zweiten 
Beobachtungsintervall eine höhere Prävalenz der Dokumentation von Kriterien wie unberechenbare und unbeständige Stimmung (98\%), wiederholte Drohungen oder Handlungen mit Selbstschädigung (94\%). Hingegen zeigt knapp die Hälfte der Patienten eine Abnahme zu Neigungen, sich auf instabile Beziehungen einzulassen (46\%), bzw. übertriebene Bemühungen, das Verlassen werden zu vermeiden (18\%) im Vergleich zum Beobachtungszeitraum 1996-2004, genauso die Gruppe der Patienten mit deutlicher Tendenz zu Streitereien und Konflikten mit anderen (28\%). Die genannten Werte ähneln der Gruppe der Patienten mit Tendenz zu unberechenbarer und unbeständiger Stimmung bzw. der Tendenz zu unerwartetemund ungeachtet der Konsequenzen gesteuertem Handeln.

Aus Abbildung 46 ist ersichtlich, dass im späteren Untersuchungsintervall eine Zunahme der Patienten bezüglich Kriterien wie anhaltende Gefühle von Leere (chi²Wert=57,805; $d f=1 ; p<0,001)$ vorliegt, gleichermaßen bei übertriebenen Bemühungen das Verlassen werden zu vermeiden (chi²-Wert=60,969; $\mathrm{df}=1 ; \mathrm{p}<0,001$ ) und bei deutlicher Tendenz zu Streitereien und Konflikten mit anderen (chi ${ }^{2}=14,040$; $d f=1$; $\mathrm{p}<0,001)$. 


\section{Abbildung 47 Vergleich der diagnostischen Kriterien nach DSM-IV (APA 1994) prozentual rot: Patienten in den Jahren 1996-2004 ( $\mathrm{N}=142)$ \\ blau: Patienten in den Jahren 2008-2012 (N=87)}

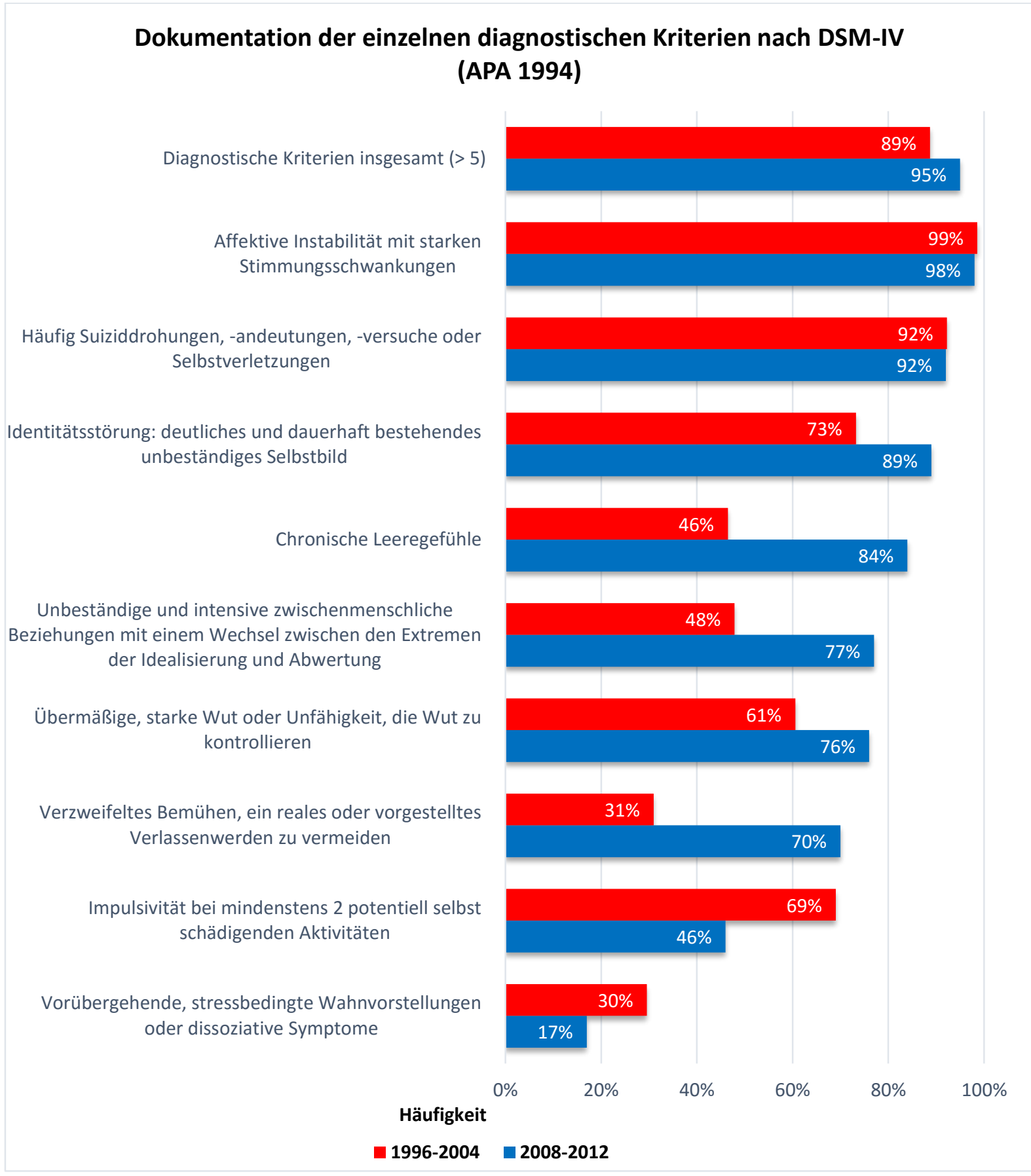




\section{Abbildung 48 Vergleich der diagnostischen Kriterien nach DSM-IV (APA 1994) statistisch rot: Patienten in den Jahren 1996-2004 ( $\mathrm{N}=142)$}

blau: Patienten in den Jahren 2008-2012 (N=87)

\section{Dokumentation der einzelnen diagnostischen Kriterien nach DSM-IV}

(APA 1994)

Diagnostische Kriterien insgesamt (> 5)

Affektive Instabilität mit starken Stimmungsschwankungen

Häufig Suiziddrohungen, -andeutungen, -versuche oder Selbstverletzungen

Identitätsstörung: deutliches und dauerhaft bestehendes unbeständiges Selbstbild

Chronische Leeregefühle

Unbeständige und intensive zwischenmenschliche Beziehungen mit einem Wechsel zwischen den Extremen der Idealisierung und Abwertung

Übermäßige, starke Wut oder Unfähigkeit, die Wut zu kontrollieren

Verzweifeltes Bemühen, ein reales oder vorgestelltes Verlassenwerden zu vermeiden

Impulsivität bei mindenstens 2 potentiell selbst schädigenden Aktivitäten

Vorübergehende, stressbedingte Wahnvorstellungen oder dissoziative Symptome

Häufigkeit
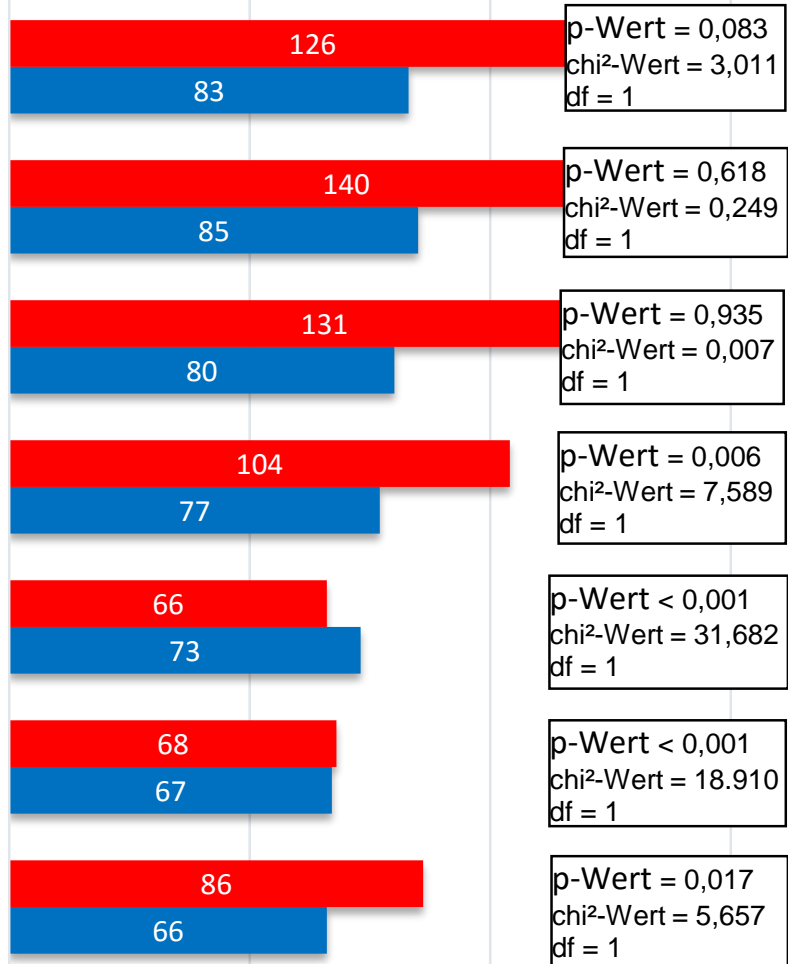

44

61

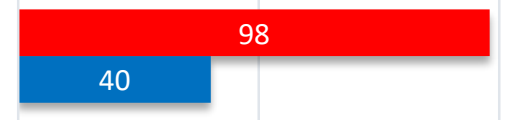

42

15

$0 \quad 50$

$50-100$

150

— 1996-2004 2008-2012

Für DSM-IV-Kriterien ergaben sich die in Abbildung 48 dargestellten Werte. Auch hier hatte es einen signifikanten Gruppenunterschied (chi²-Wert=31,652; $d f=1 ; p<0,001)$ in der Beschreibung chronischer Leeregefühle; unbeständiger und intensiver zwischenmenschlicher Beziehungen; ein Verlassenwerden zu vermeiden und Impulsivität bei mindestens zwei potentiell selbstschädigenden Aktivitäten zum Ergebnis. Insgesamt ist ähnlich wie bei den Ergebnissen der Symptome nach ICD-10 
eine quantitativ bessere Dokumentation von klassifikatorischen Symptomen zu erkennen.

\subsubsection{Veränderung der Psychopharmakotherapie zum Zeitpunkt der Entlassung}

Abbildung 49 Vergleich der Einnahme von Neuroleptika bei Entlassung prozentual rot: Patienten in den Jahren 1996-2004 ( $\mathrm{N}=53)$

blau: Patienten in den Jahren 2008-2012 (N=40)

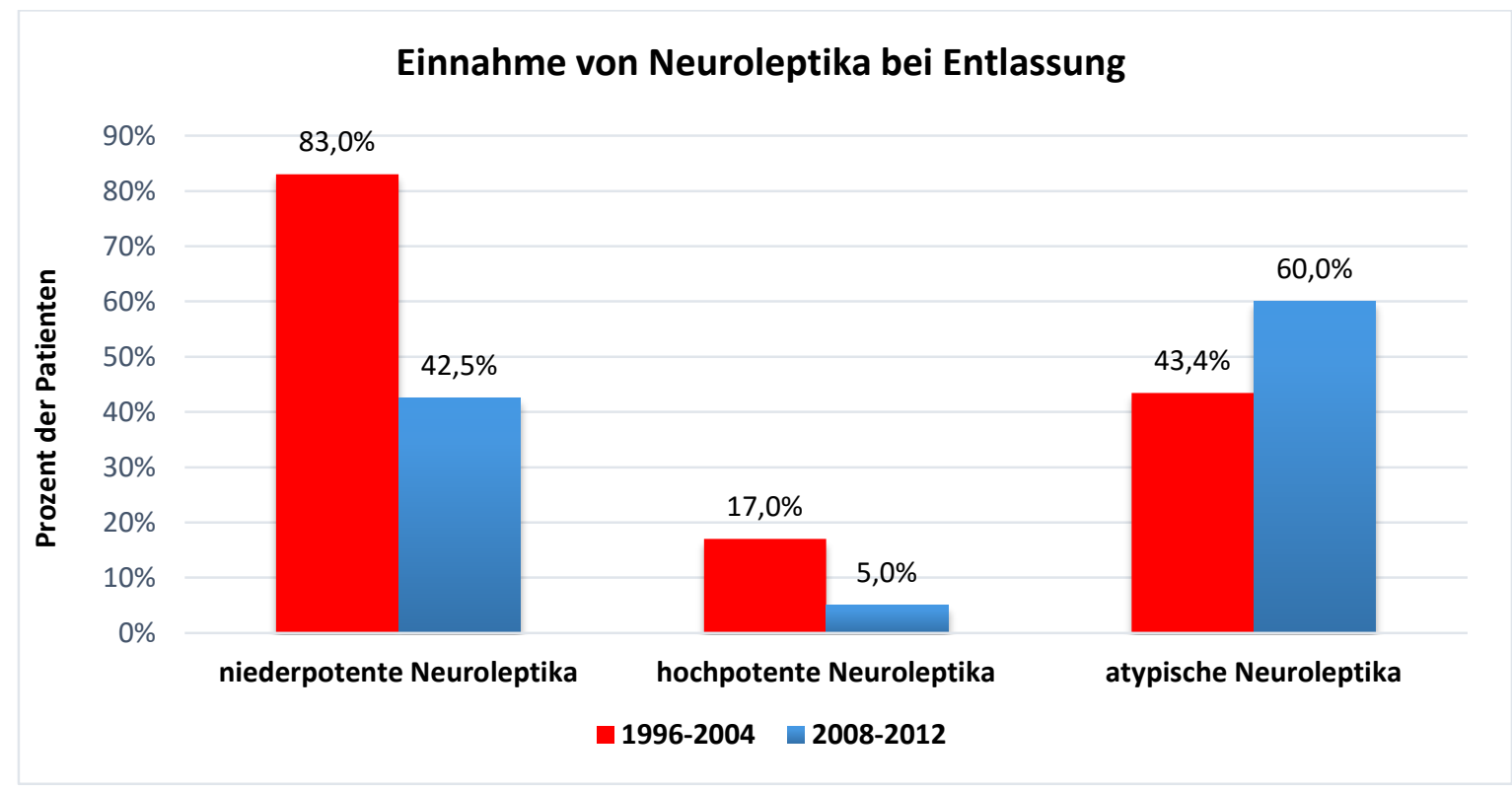

Abbildung 50 Vergleich der Einnahme von Neuroleptika bei Entlassung statistisch rot: Patienten in den Jahren 1996-2004 ( $\mathrm{N}=53)$

blau: Patienten in den Jahren 2008-2012 ( $\mathrm{N}=40)$

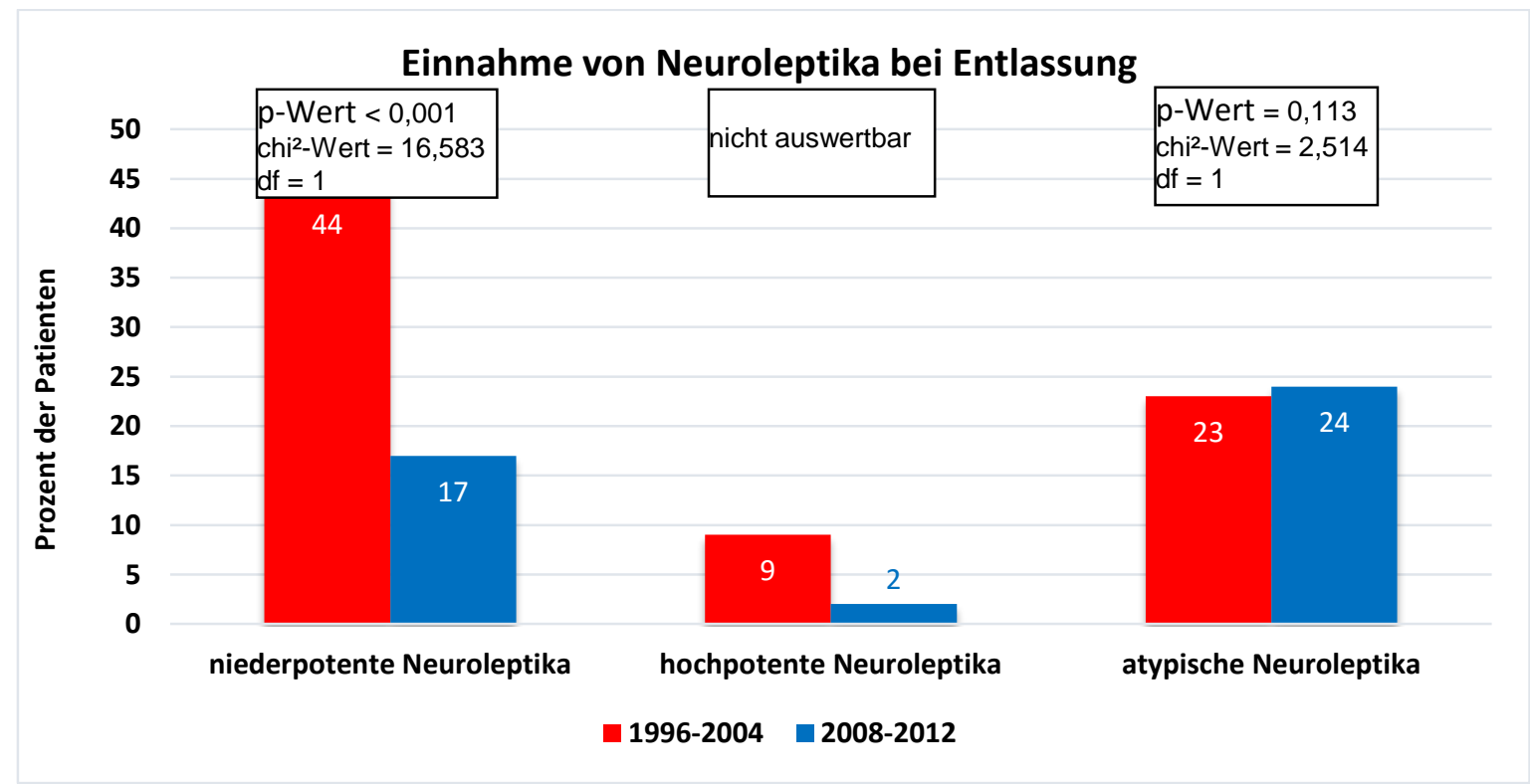


Während es bei atypischen Neuroleptika keine signifikanten Unterschiede in der Häufigkeit ihrer Verschreibungen zwischen den Untersuchungszeiträumen gab, wurden niederpotente Neuroleptika hochsignifikant seltener im Zeitraum 2008-2012 während der Kliniksaufenthalte bis zur Entlassung verordnet (chi²-Wert=16,583; df=1; p-Wert<0,001). Bei hochpotenten Neuroleptika ist aufgrund der Nichtauswertbarkeit keine Aussage möglich.

\section{Abbildung 51 Vergleich Psychopharmakahauptgruppen bei Entlassung prozentual rot: Patienten in den Jahren 1996-2004 ( $\mathrm{N}=102)$ blau: Patienten in den Jahren 2008-2012 (N=82)}

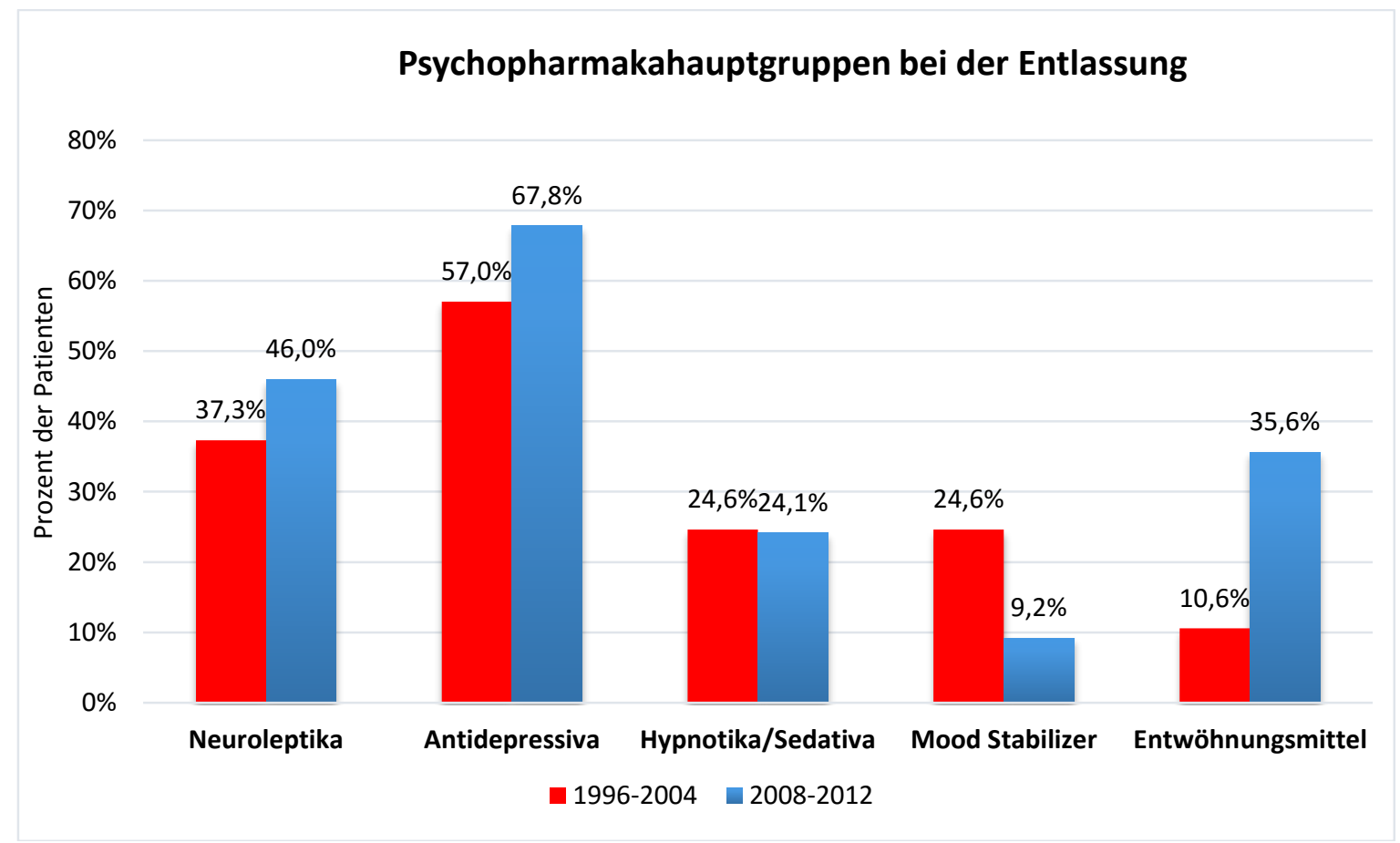


Abbildung 52 Vergleich Psychopharmakahauptgruppen bei Entlassung statistisch rot: Patienten in den Jahren 1996-2004 $(\mathrm{N}=102)$ blau: Patienten in den Jahren 2008-2012 (N=82)

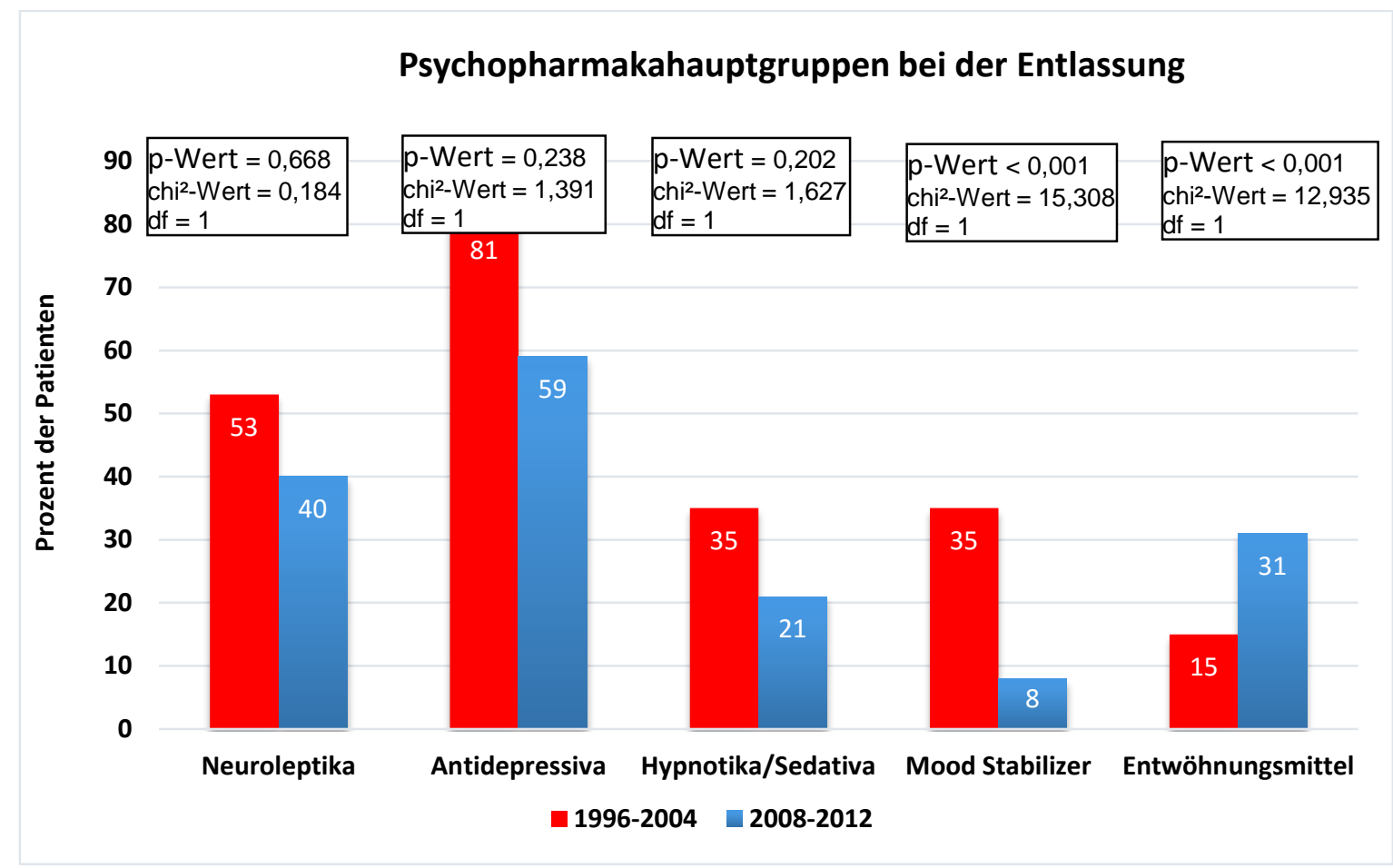

Während sich eine signifikante Abnahme bei der Verordnung von Mood-Stabilzern zeigte $\left(p<0,001 ;\right.$ chi $\left.^{2}=15,308, d f=1\right)$, blieb die Anzahl der Verabreichung von Sedativa und Hypnotika über die Jahre etwa gleich. Ein signifikanter Anstieg in der Verordnung von Entwöhnungsmitteln, die am ehesten auf die häufige Verordnung von Naltrexon zurückzuführen ist, wird über den zeitlichen Verlauf sichtbar $\left(\operatorname{chi}^{2}=12,935\right.$; $d f=1$; $p<0,001)$. 
Abbildung 53 Vergleich Patienten, die bei der Entlassung Antidepressiva bekamen prozentual rot: Patienten in den Jahren 1996-2004 (N=80)

blau: Patienten in den Jahren 2008-2012 (N=59)



Abbildung 54 Vergleich Patienten, die bei der Entlassung Antidepressiva bekamen statistisch rot: Patienten in den Jahren 1996-2004 (N=80)

blau: Patienten in den Jahren 2008-2012 (N=59)

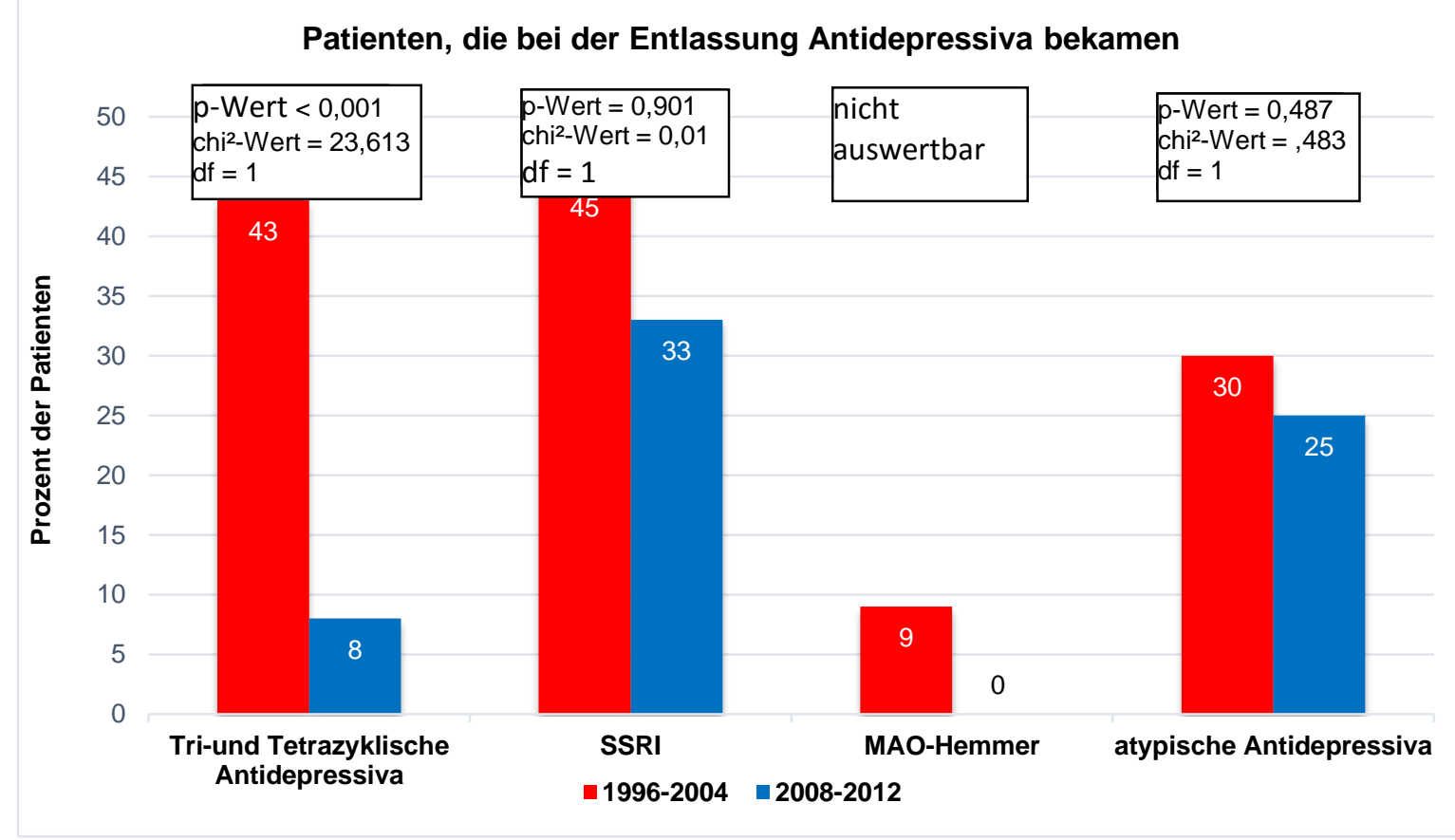

Innerhalb der Antidepressiva-Verordnungen zeigte sich, dass im zweiten Beobachtungzeitraum signifikant weniger tri- und -tetrazyklische Antidepressiva eingesetzt wurden $\left(\mathrm{chi}^{2}=23,613 ; \mathrm{df}=1 ; \mathrm{p}\right.$-Wert $\left.<0,001\right)$. Gleich geblieben scheint die 
Substanzklasse von der SSRI, auffällig ist ein komplettes Fehlen der Verordnung von MAO-Hemmer in den Jahren 2008-2012. 


\section{Diskussion}

Die vorliegende Arbeit befasst sich mit der Untersuchung der klinischen Realität und Praxis der stationären Psychopharmakotherapie bei der Behandlung von Patienten mit einer emotional instabilen PS vom Borderline Typ an einer Universitätsklinik.

In der vorliegenden Studie konnte gezeigt werden, dass 94\% der BPS Patienten während stationärer Behandlungen Psychopharmaka bekommen.

Wie Patienten mit anderen psychiatrischen Störungen werden in der Realität BPSPatienten häufig mit Psychopharmaka behandelt. Allerdings scheint die Psychopharmakotherapie zu nur unbefriedigenden Resultaten zu führen, da eine stabile Remission selten erreicht wird und Rehospitalisierungen oft nicht zu verhindern sind.

Die Neurobiologie von BPD bleibt unklar. In einer Übersichtsarbeit von Bandelow und Wedekind (2010) wurde eine Theorie von Abänderungen in der Sensitivität von opioiden Rezeptoren im ZNS präsentiert. Die alarmierenden Symptome und selbstzerstörende Verhaltensweisen von den betroffenen Patienten können durch unkontrollierbare und gewissenslose Versuche erklärt werden, ihr endogenes Opiodsystem (EOS) und das dopaminergische Belohnungssystem zu stimulieren, ungeachtet der schädlichen Konsequenzen. Erkenntnisse die diese Hypothese unterstützen, wurden überprüft. Aufmerksamkeitssuchendes Verhalten kann durch den Versuch erklärt werden, Nutzen von belohnenden Effekten der menschlichen Bindung, vermittelt durch EOS, zu ziehen. Anhedonie und Gefühle von Leere können Ausdruck von reduzierter Aktivität des EOS sein. Patienten mit BPD tendieren zum Missbrauch von Substanzen die auf mu-opioide Rezeptoren abzielen.

Insgesamt müssen die Erfolgsquoten jeglicher Behandlung der BPS allerdings bisher als unbefriedigend angesehen werden. Trotz langer ambulanter und stationärer Behandlungen kommt es immer wieder zur krisenhaften Zuspitzung von BPSSymptomatik mit Selbstverletzungen, Suizidversuchen, Drogenrückfällen etc. Neurobiologische Studien nehmen an, dass Abnormalitäten in fronto-limbischen Netzwerken mit vielen dieser Symptome verknüpft sind (Leichsenring et al. 2011).

Obgleich also ein hoher Prozentsatz der BPS-Patienten Medikamente verordnet bekommt, werden offene und kontrollierte Medikamentenstudien bei der BPS im 
Vergleich zu anderen psychiatrischen Störungen seltener durchgeführt, die Anzahl aussagekräftiger Übersichtsarbeiten ist klein. Hinzu kommt, dass die vorhandenen klinischen Pharmakastudien in aller Regel an kleinen Fallzahlen bei heterogener Zielsymptomatik und mit unterschiedlichen Ein- und Ausschlusskriterien mangeln. Für eine chronische Krankheit wie die BPS war die Dauer der Studien zudem oft sehr kurz. So existieren bislang kaum dezidierte Leitlinien zur Hilfe bei der Auswahl einer individuellen und vernünftigen - die psychotherapeutische Intervention unterstützenden - psychopharmakologischen Behandlung. Leitlinien wie die englischen NICEGuidelines verzichten völlig auf die Empfehlung von Psychopharmaka in der BPS Behandlung, während die Amerikanische Psychiatrische Gesellschaft APA eine Symptom- bzw. Syndrom orientierte Pharmakotherapie präferiert.

Die Ergebnisse der vorliegenden Studien zeigen signifikante Effekte an Kernsymptomen von BPD, trotzdem gibt es offensichtlich erhebliche Grenzen bei der Vergleichbarkeit und Aussagekraft innerhalb der Studien aufgrund der Heterogenität in den Patientengruppen, additiver Medikation und Maßnahmen (Dell'Osso und Altamura 2010).

Bislang gibt es nur wenige Studien die sich mit medikamentösen Langzeitbehandlungsverläufen von BPS-Patienten beschäftigen.

\subsection{Psychopharmakotherapie allgemein}

In der vorliegenden Studie konnte gezeigt werden, dass 67\% der Patienten schon bei der Aufnahme Psychopharmaka bekamen. Dieses Ergebnis bestätigte die klinische Erfahrung, dass BPS-Patienten häufig langfristig psychotrope Medikamente bekommen. Verglichen mit den Ergebnissen aus anderen Studien bestätigt dieses Ergebnis bisherige Prozentzahlen über die Häufigkeit einer Psychopharmakotherapie. Andrulonis et al. (1982) fanden in einer Untersuchung von 102 Borderline-Patienten bei $85 \%$ der Patienten eine stationäre medikamentöse Vorgeschichte. Eine neuere Studie von Zanarini et al. (2004) mit 290 Borderline-Patienten ergab bei 84,1\% der stationären Patienten Erfahrungen mit Psychopharmaka, die sich allerdings auch auf ambulante Verschreibungen bezogen. Heinze et al. (2005) fanden bei 84\% aller Aufenthalte bei Patienten mit Persönlichkeitsstörungen einen Einsatz von Psychopharmaka. 
Maladaptive BPS-Symptome sprechen auf einzelne Antidepressiva, Antipsychotika, Mood Stabilizer, aber auch Omega-3-Fettsäuren und andere Medikamente an (Ripoll et al. 2011). Evidenz basierte Pharmakotherapie ist am nützlichsten beim Behandeln von begrenzten Symptomgebieten, erzeugt aber nur eine partielle Verbesserung von Persönlichkeitsstörungen (Ripoll et al. 2011).

Auf eine hohe Kontinuitätsrate der Psychopharmakotherapie bei der Aufnahme und Entlassung lässt (1) schließen, dass 67\% Patienten schon Erfahrung mit Psychopharmaka hatten und (2), dass die vorwiegend verabreichten Substanzklassen Antidepressiva und Neuroleptika über jeweils knapp die Hälfte der Gesamtbehandlungsdauer gegeben wurden. Es ist zu vermuten, dass auch außerhalb des Krankenhauses oft eine durchgängige Psychopharmakotherapie erfolgte.

Es gibt zunehmende Hinweise darauf, dass Antiepileptika und Antipsychotika der zweiten Generation, die derzeitig bevorzugte Medikation zur Behandlung für BPD Symptome sind und dass diese auch das Verlangen und den Konsum von Alkohol reduzieren konnten (Gianoli et al. 2012).

Eine Pharmakotherapie scheint in der Zeit von akuteren Beschwerden zudem notwendig zu sein.

Nach Literatursichtung wurden unserem Wissen nach erstmals Unterschiede in der Psychopharmakotherapie der BPS bei verschiedenen Behandlungsmodi untersucht. Überraschenderweise ließen sich keine signifikanten Unterschiede in der Häufigkeit einer verordneten Psychopharmakotherapie bei Aufenthalten zur Krisenintervention oder Psychotherapie bei der Entlassungsmedikation und während stationärer Behandlung finden. Dies widerspricht der Ansicht von Dulz und Schneider (1996), die eine über Kriseninterventionen hinausgehende Therapie mit Psychopharmaka für nicht indiziert ansehen und zeigt den zum Teil erheblichen Widerspruch zwischen Theorie und Praxis.

Im Laufe eines Aufenthaltes, von der Aufnahme- zur Entlassungsmedikation, an der Klinik für Psychiatrie und Psychotherapie an der Universität Göttingen, nahm die Anzahl der verabreichten Psychopharmaka signifikant zu. Diese Steigerung betraf alle Substanzklassen mit Ausnahme der Hypnotika und Sedativa (vor allem die Benzodiazepine), die im Laufe eines Aufenthaltes in ihrer Anzahl um 5,8\% reduziert 
wurden. Dies spricht für ein allgemein zunehmendes Problembewusstsein in der Langzeitverschreibung von Benzodiazepinen.

Am stärksten nahm die Verordnung von Antidepressiva $(+17,2 \%)$ und Neuroleptika $(+11,5 \%)$ zu, hier waren es vorwiegend die niederpotenten und atypischen Substanzen, die im Laufe der stationären Behandlung zusätzlich verordnet wurden.

Die Verordnung der Psychopharmaka nahm von der Vor- zur Entlassungsmedikation um $27 \%$ zu. Die größte Steigerung erfuhren Antidepressiva und Entwöhnungsmittel wie Naltrexon.

Im Zeitverlauf zeigte sich, dass die Patienten im Zeitraum 2008-2012 mehr verschiedene Medikamente verordnet bekamen als im Zeitraum 1996-2004. Bei der Entlassung ist der Anteil von Antidepressiva gestiegen von 50,6\% (1996-2004) auf 67,8\%. (2008-12). Antipsychotika wurden auch nominell gesteigert auf 46\%. Dies bestätigen die Ergebnisse von Heinze et al. (2005), die bei einem Vergleich der Verschreibungspraxis von 1996 und 2003 die stärkste Zunahme bei den Antidepressiva und Antikonvulsiva fanden. Die Therapie affektiver Symptome könnte somit an Bedeutung gewonnen haben.

Aus der häufigen Medikamentengabe kann die Schlussforderung gezogen werden, dass eine Pharmakotherapie bei BPS offensichtlich sehr oft als notwendig erachtet wurde. Schwerwiegende psychopathologische Krankheitszeichen wie unkontrollierbare Impulsivität, affektive oder psychotische Symptome erfordern wiederholt den gezielten Einsatz von Psychopharmaka. Wenn man bedenkt, dass die Hälfte der Patienten mit suizidalen Gedanken oder aufgrund von Selbstverletzungen, parasuizidalen Handlungen oder Suizidversuchen in ärztliche Behandlung kamen, scheint eine Psychopharmakotherapie auch wichtig zu sein, um akut zu stabilisieren und antisuizidal zu wirken. Somit sind die vorliegenden Empfehlungen zur symptombzw. syndromorientierten Pharmakotherapie bei der BPS zunehmend umgesetzt worden.

\subsection{Psychopharmakotherapie speziell}

Eine genauere Betrachtung der verabreichten Substanzklassen bei der Aufnahme konnte zeigen, dass vorwiegend Antidepressiva zum Einsatz kamen, gefolgt von 
Neuroleptika (Erfahrung bei $40 \%$ der Patienten) sowie Hypnotika und Sedativa (Erfahrung bei 29\% der Patienten).

\section{- Antidepressiva}

Die Hälfte der Patienten (50,6\%) hatte vor der Aufnahme bereits Erfahrung mit Antidepressiva gemacht. Im Vergleich zu der Studie von Bender et al. (2001), die bei 61\% aller Patienten Erfahrungen mit Antidepressiva fanden, wurden in der vorliegenden Studie dementsprechend noch etwas mehr Antidepressiva im Vorfeld verordnet. Zanarini et al. (2001) dagegen zeigten bei $80 \%$ der Patienten Antidepressiva in der Vorgeschichte, dieses Ergebnis bezieht sich allerdings auf ambulante und stationäre Verschreibungen.

Antidepressiva wurden erwartungsgemäß und analog der Literaturempfehlungen vor allem bei einer affektiven Symptomatik verordnet, aber auch bei Suizidalität oder nach erfolgten Suizidversuchen.

Am häufigsten eingesetzt wurden hierbei Antidepressiva aus der Gruppe der SSRI und etwas weniger tri-und tetrazyklische Antidepressiva, die Rangfolge kehrt sich allerdings um, vergleicht man beide hier unterschiedliche Zeiträume: Zwischen 19962004 und 2008-2012 lässt sich eine starke Zunahme der SSRIs beobachten, bei gleichzeitiger Abnahme der Verschreibung tri- und tetrazyklischen Substanzen (von $53,8 \%$ auf 13,6\% der Aufenthalte). Diese Entwicklung ist konform mit den heutigen Empfehlungen und Leitlinien, in denen den SSRIs eine immer größere Bedeutung zukommt, unter anderem da sie weniger Nebenwirkungen als die trizyklischen Antidepressiva haben und entsprechende Positivstudien vorliegen. Eine langsame Dosierung kann den Wirkungseintritt verzögern. Zum Beenden sollten TZA langsam abgesetzt werden, sonst kommt es zum Wiederauftreten von Symptomen. Um die Dosierung besser steuern zu können, muss die Konzentration im Plasma überprüft werden. SSRI werden bei BPS als effektiv beschrieben bei abfallendem Schwergrad von depressiver Stimmung, Angst und Ärger Bellino et al. (2011). Auch im Hinblick auf Überdosierungen in suizidaler Absicht sind die SSRls erheblich sicherer als die trizyklischen Antidepressiva.

Dennoch ist der Einsatz tri- und tetrazyklischen Substanzen noch bemerkenswert häufig, wenn man bedenkt, dass es zu dieser Substanzklasse im Gegensatz zu den SSRIs kaum Studien gibt, die deren Wirksamkeit bei der BPS belegen. Damit bestätigt 
sich die Erfahrung von Heinze et al. (2005), die zum einen die hohe Verschreibungspraxis von Antidepressiva bei Persönlichkeitsstörungen feststellen konnten (in 61\% aller Aufenthalte 2003), zum anderen auch den im Vergleich zu SSRIs relativ häufigen Gebrauch von trizyklischen Antidepressiva konstatierten. Allerdings zeigte auch diese Untersuchung eine starke Abnahme des Einsatzes von trizyklischen Antidepressiva im Zeitverlauf von 1996-2004. Eine andere Studie von Zanarini et al. (2004a) zeigte, im Gegensatz zu der vorliegenden Studie, bereits in den neunziger Jahren eine Dominanz der SSRIs gegenüber den trizyklischen Substanzen bei dieser Indikation.

Die am häufigsten verwendeten Antidepressiva waren Trimipramin, Doxepin, Mirtazapin, gefolgt von den SSRIs Citalopram und Fluvoxamin. Eine mögliche Erklärung für die weitere hohe Verordnung von TZA mögen deren deutliche sedierende Effekte sein, die somit zunächst akut hilfreich erscheinen, jedoch keine evidenzbasierte Verordnungsrationale haben.

\section{- Neuroleptika}

Überraschend hoch war der Anteil an verabreichten Neuroleptika. Schon vor dem stationären Aufenthalt hatte knapp die Hälfte der Patienten im Untersuchungsraum 2008-2012 mindestens einmal Neuroleptika bekommen. Es wurden Neuroleptika auch häufig bei affektiven und impulsiven Syndromen gegeben, bei denen gemäß den Empfehlungen allerdings SSRIs, also Antidepressiva, die erste Wahl wären. Hauptsächlich wurden atypische Neuroleptika eingesetzt. Im Vergleich zu den Jahren 1996-2004 zeigte sich eine Abnahme von Verschreibungen nieder-und hochpotenter Neuroleptika. Dafür wurden häufiger atypische Neuroleptika verordnet. Es ist zu vermuten, dass diese gerade bei impulsiven Patienten zur Sedierung oder, wie für Olanzapin beschrieben, zur Stimmungsstabilisierung dienten. Ebenso dürften Patienten mit selbst verletzendem Verhalten, bei denen Neuroleptika auch sehr häufig zum Einsatz kamen, mit Hilfe niederpotenter Neuroleptika, aber auch mit Hilfe von Hypnotika und Sedativa, beruhigt worden sein. Wie schon von Heinze et al. (2005) beschrieben, kam es vielfach zu einem Einsatz älterer Neuroleptika, was überraschend ist, da es kaum studienbasierte Empfehlungen für diese Substanzen gibt und Nebenwirkungen häufiger sind als bei neueren Substanzen. 
Im Vergleich zu anderen Studien wurden in dieser Studie insgesamt häufiger Neuroleptika verschrieben: Nur 10\% der Patienten hatten in der Studie von Bender et al. (2001) irgendeine Vorerfahrung mit dieser Substanzklasse, im Gegensatz zu 34,5\% in der vorliegenden Studie. Zanarini et al. (2001) fanden in den USA ebenso bei 38,6\% eine ambulante oder stationäre Vorerfahrung mit Neuroleptika. In einer neueren Studie von Heinze et al. (2005) wurde für das Jahr 2003 bei $60 \%$ der stationären Aufenthalte ein Einsatz von Neuroleptika bei Patienten mit einer Persönlichkeitsstörung beschrieben.

Betrachtet man die Veränderungen innerhalb der Neuroleptikagruppe, fällt vor allem eine starke Zunahme der atypischen Neuroleptika auf, einhergehend mit einer Abnahme der Verordnungen von niederpotenten Neuroleptika. Atypische Neuroleptika etablierten sich erst im Laufe der neunziger Jahre, was diese Entwicklung erklären dürfte. Außerdem ist die Wirksamkeit der atypischen Neuroleptika, vor allem von Olanzapin, bei der BPS in mehreren Studien belegt worden (Tabelle 2), bei besserer Verträglichkeit als die alten Substanzen. Eine Übersichtsarbeit über fünf placebokontrollierte Studien zu Lamotriginin in der akuten Behandlung zeigt nach differenzierter Betrachtung unterschiedlicher Schwergrade der bipolaren Störung, eine signifikante Überlegenheit für Lamotrigin. Nur bei schweren depressiven Episoden soll langsam dosiert werden, unter Kontrolle der Plasmakonzentration. Lamotrigin wirkt möglicherweise suchtfördernd. Beim Einsatz von Antikonvulsiva sollte sorgfältig auf das Suizidrisiko geachtet werden.

Antipsychotika der zweiten Generation verbessern signifikant kognitive Symptome bei Patienten mit BPS, so dass dementsprechend ein Einsatz empfohlen wird (Bellino et al. 2011). Vorteil dieser Substanzen ist weiterhin eine sehr geringe Rate an extrapyramidal-motorischen Nebenwirkungen. Bei den hochpotenten Substanzen gab es im Zeitverlauf wenige Veränderungen.

Die am häufigsten verwendeten Neuroleptika waren Quetiapin und Olanzapin, sowie Perazin, Promethazin und Pipamperon.

\section{- Hypnotika/ Sedativa}

In Anbetracht der empirischen Studienlage war der häufige Gebrauch von Hypnotika und Sedativa überraschend. Knapp die Hälfte der Patienten hatte Erfahrung mit Benzodiazepinen während früherer stationärer Behandlungen. Nach heutigen 
Richtlinien sollten diese allerdings mit Ausnahme von Kriseninterventionen nicht mehr gegeben werden. Insbesondere bedingt durch ihr Abhängigkeitspotential und Berichten über eine Zunahme von Kontrollverlusten sind sie grundsätzlich als obsolet zu betrachten (Schmahl und Bohus 2001). So konstatierte z.B eine Studie von Gardner und Cowdry (1985) bei BPS-Patienten unter Alprazolam-Therapie eine erhöhte Suizidalität und eine Zunahme aggressiver Verhaltensweisen.

Bei der Interpretation ist aber gleichwohl zu beachten, dass diese Medikamentenklasse während der stationären Aufenthalte in Quantität und Dosierung verringert wurde.

Die Indikation für den Einsatz von BZD ist zumeist symptomorientiert. In vielen Fällen erfolgt der Einsatz um den Therapieeffekt zu unterstützen oder die Wirklatenz einer anderen, längerfristig geplanten Medikation zu unterstützen. BZD sind bei vielen psychiatrischen und internistischen Notfallsituationen indiziert. Anders als bei Antidepressiva gibt es keine neuen Zulassungsstudien für BZD, die sich auf die ICD10 Klassifikation beziehen. Es handelt sich in der Regel um Altzulassungen mit unspezifischen Syndromnennungen.

Das mit Abstand am häufigsten verwendete Hypnotikum/Sedativum war Diazepam. Es scheint also grundsätzlich so zu sein, dass Patienten mit einer vorhergehenden sedativen Medikation in die Klinik kommen, diese dann aber langsam versucht wird auszuschleichen.

Interessant erscheint, dass 1983 in einer Studie von Skodol et al. Benzodiazepine die am häufigsten verschriebene Substanzklasse waren, was sich in der relativ hohen Erfahrungsrate mit Diazepam (37\% der Patienten) und Chlordiazepoxid (20\% der Patienten) widerspiegelte. An zweiter Stelle rangierten antipsychotische Substanzen wie Thioridazin (bei 13\% der Patienten Vorerfahrungen), Antidepressiva waren von untergeordneter Bedeutung. Die vorliegende Studie bestätigt den deutlich veränderten Psychopharmakagebrauch in den letzten dreißig Jahren.

\section{- Opiatantagonisten}

Betrachtet man die Veränderungen zwischen den Gruppen, fällt vor allem eine starke Zunahme der Verordnungen von Naltrexon auf über $25 \%$ auf. BPD-Symptome, wie Substanzmissbrauch, Anorexie, Selbstverletzung, Depersonalisation und sexuelle 
Überstimulation, können erfolgreich mit Antagonisten an opiodergen Rezeptoren behandelt werden. Ein Verständnis in Neurobiologie von BPD könnte beim Entwickeln von neuen Behandlungen für Patienten mit ernsten Störungen von Vorteil sein (Bandelow, et al. 2010), da diese Substanz möglicherweise auch affektregulierende Eigenschaften hat.

Der Opiatantagonist Naltrexon wird normalerweise zur Entwöhnungsbehandlung Opiatabhängiger nach erfolgter Opiat-Entgiftung eingesetzt. Bei Patienten mit BPS konnte Naltrexon vor allem eine Abnahme von selbst verletzendem Verhalten bewirken (z.B. Griengl und Dantendorfer 2001). Problematisch ist hierbei die relativ hohe Opiatabhängigkeitsrate bei BPS-Patienten. Ist der Patient nicht opiatfrei, kommt es zu einer Entzugssymptomatik.

Im Fazit zeigte sich eine sehr heterogene Verschreibungspraxis. Medikamente wurden aus allen psychotropen Substanzklassen und in unterschiedlichsten Dosierungen eingesetzt. Dies verdeutlicht zum einen eine allgemeine Unsicherheit in der pharmakologischen Therapie, teils bedingt durch fehlende Evidenz basierte Medikamentenrichtlinien, zum anderen ist dies ein Resultat der problematischen Therapie eines multidimensionalen Syndroms mit heterogener Symptomatik und vielen Komorbiditäten. Offensichtlich gibt es in der klinischen Praxis nicht „das Borderline-Medikament" und es existiert auch keine entsprechende Zulassung.

Ob eine Pharmakotherapie entlang der vorhandenen, noch wenig etablierten Richtlinien erfolgte, ließ sich aufgrund von häufigen Medikamentenwechseln in einer längeren Behandlungsgeschichte und individuellen Verläufen schwierig nachvollziehen und scheint eher nicht wahrscheinlich. Meistens wurden jedoch bei den entsprechenden vorherrschenden Syndromkomplexen die jeweils empfohlenen Substanzklassen gegeben, z.B. Antidepressiva bei affektiven Symptomen und Neuroleptika bei psychotischen Symptomen. Bei Patienten mit einem impulsiven Syndromkomplex schien allerdings eine gewisse Unsicherheit vorzuherrschen, jene wurden mit allen Substanzklassen therapiert, keine Klasse dominierte deutlich. Entsprechend den vorhandenen Leitlinien wären Antidepressiva bei impulsiver Symptomatik die erste Wahl. Auffallend waren demzufolge die hohen Erfahrungsraten mit Hypnotika und Sedativa, die in den Leitlinien bei impulsiver Symptomatik nicht empfohlen werden, und mit Neuroleptika, die aber zumindest als 2. Wahl gelten. Beide 
Substanzklassen wurden bei impulsiven Patienten ähnlich häufig gegeben wie Antidepressiva.

Eine routinemäßige und Langzeitverordnung vielfältiger psychotroper Medikamente, die gewöhnlich geringeren und manchmal unsicheren Erfolg zeigten und auf lange Sicht zum Teil abhängigkeitserzeugend sind, ist folglich eine Realität in der modernen psychiatrischen Behandlungspraxis für Patienten mit einer BPS.

\subsection{Demographische Gesichtspunkte}

Die BPS ist eine Störung der Stress- und Affektregulationen sowie der Impulskontrolle. Die Entwicklung von interpersonellen, emotions- und selbstregulativen Bewältigungsstrategien ist beeinträchtigt und oftmals wird die Ausbildung dieser Fähigkeit durch soziale Umgebungsfaktoren weiter gestört bzw. ihr Einsatz unterbunden .Dennoch ist die BPS durch ein umfassendes Therapiekonzept langfristig behandelbar. Die Erkrankung verursacht umfassende soziale Probleme wie häufige Konflikte, unstete Bindungen, inadäquate Ausbildung und Arbeitslosigkeit. Die Suizidrate ist sehr hoch und liegt bei $5-10 \%$.

Aktuelle Langzeitstudien zeigen, dass die Prognose der BPS besser ist als in den letzten Jahrzehnten angenommen. In einer Studie von Zanarini et al. (2003) trafen nach 10 Jahren nur noch auf $12 \%$ der Patienten mit ursprünglich diagnostizierter BPS die DSM-IV-Kriterien dieser Störung. Als geheilt wurden Probanden eingestuft, die neben einer Symptomfreiheit auch sozial und beruflich ein stabiles Funktionsniveau wiedererlangt hatten. Dies stellt die Wichtigkeit einer umfassenden sozialen und beruflichen Integration für die Nachhaltigkeit einer einmal erzielten Remission.

Im untersuchten Zeitraum 2008-2012 zeigte sich nach wie vor eine hohe Inanspruchnahme stationärer Versorgung. Bezogen auf alle auswertbaren Aufenthalte ergab sich eine mittlere Aufenthaltsdauer von 22,7 Tagen pro Jahr. Diese Angaben liegen deutlich unter den Daten einer Studie von Jerschke et al. (1998), die zeigte, dass die BPS-Patienten eine mittlere Liegezeit von 45 Tage pro Jahr hatten.

Im Zeitverlauf zwischen 1996-2004 und 2008-2012 ergab sich hinsichtlich der durchschnittlichen Aufenthaltsdauer aller Aufenthalte eine Diskrepanz; bei der Analyse aller Aufenthalte nahm die Aufenthaltsdauer im letzten Intervall um die Hälfte ab. 
Hieraus lassen sich schwer Schlüsse ziehen; möglich ist, dass geringe Fallzahlen im zweiten Beobachtungszeitraum ein Missverhältnis bewirkten. Andererseits könnte spekuliert werden, dass die Kriseninterventionen und möglicherweise auch die Psychotherapien konzertierter und effizienter geworden sind und eine raschere Entlassung somit möglich war. Eventuell könnte auch die gezieltere Pharmakotherapie hierfür kennzeichnend sein.

Die Borderline-Patienten in der Untersuchungsgruppe wiesen viele psychiatrische Komorbiditäten auf, allerdings stellen diese meist keine Komorbiditäten im eigentlichen Sinne dar, sondern sind vielmehr als syndromale Merkmale bzw. Folgen der BPS aufzufassen. Auffallend war der hohe Prozentsatz von 40\% der Patienten mit psychischen- und Verhaltensstörungen durch psychotrope Substanzen, vor allem alkoholbezogene Störungen, der auch im Vergleich mit anderen Studien auffällt. Zimmermann und Mattia (1999) fanden nur bei $13 \%$ der von innen untersuchten Patienten einen Alkohol- oder Drogenmissbrauch, Zittel-Conklin und Westen (2005) bei immerhin 35,6\%, Zanarini et al. (2004b) bei $62,1 \%$. Affektive Störungen waren dagegen in unserem Patientenkollektiv wesentlich geringer vertreten als in anderen Studiengruppen (31\% im Vergleich zu über 90\% bei Zittel-Conklin und Westen 2005 oder Zanarini et al. 1998). Die Häufigkeit von Essstörungen lag mit einer Prozentrate von $14 \%$ im Rahmen anderer Studien (24,4\% bei Zittel-Conklin und Westen 2005; 53\% bei Zanarini et al. 1998), ebenso wie die Häufigkeit von Posttraumatischen Belastungsstörungen (40\% im Vergleich zu 31,1\% bei Zittel-Conklin und Westen 2005; $55,9 \%$ bei Zanarini et al. 1998).

Vergleicht man die vorliegende Studie 2008-2012 mit der Studie von Will und Malevani (Gescher et al. 2013) die im Zeitraum von 2005-2009 im LVR- Klinikum Düsseldorf durchgeführt wurde und das soziodemographischen Profil von BPS Patienten analysierte, stellt man fest, dass das Alter der Patienten mit der Diagnose F 60.3 in beiden Grippen bei Aufnahme durchschnittlich 30,5 Lebensjahre betrug.

Die "Wohnsituation bei der Aufnahme“ zeigte, dass die Patienten zum größten Teil in selbstständiger Wohnform leben.

Das Merkmal "Höchster erreichter Schulabschluss“ zeigte in unserer Studie gleich starke Anteile für den Hauptschulabschluss und die Fachoberschulreife $(39,5 \%$ bzw. 30,9\%). 18,5\% der Patienten hatten inr Abitur abgeschlossen. Ähnlich fiel der Anteil 
der Patienten mit abgeschlossener Fachoberschulreife (25,5\%) bei Will und Malevani (Gescher et al. 2013) aus, bei einem Anteil an abgeschlossenem Abitur von 16,2\%.

Eine abgeschlossene Berufsausbildung gaben 24,7\% der Patienten mit der Diagnose F 60.3 an. In der Studie von Will und Malevani (Gescher et al. 2013) sind es 36,1\%.

Die Anzahl von Patienten mit abgeschlossenem Hochschulstudium fiel in der Göttinger Patientengruppe mit 3,7\% etwas geringer als in der Studiengruppe aus Düsseldorf aus.

Nach Samuels et al. (2002) sind Persönlichkeitsstörungen im Allgemeinen, so auch die BPS, assoziiert mit einer niedrigeren sozialen Schicht. Auch Swartz et al. (1990) attestierten den Borderline-Patienten einen niedrigeren sozioökonomischen Status, analog fand sich in unserer Patientenstichprobe häufig eine Kindheit in mittleren bis unteren sozialen Schichten.

Patienten mit BPS machen seltener Abitur, haben seltener einen Hochschulabschluss, haben aber insgesamt häufiger einen Schulabschluss sowie eine abgeschlossene Ausbildung (Berufsausbildung und Fach-/Hochschulabschluss zusammen).

Diese Ergebnisse lassen sich gut vereinbaren mit der Tatsache, dass die ersten Symptome der BPS bereits im Schulalter einsetzen, diese jedoch in der Regel nicht zur Unterbrechung der Schulzeit führt, sondern lediglich zu schlechteren Leistungen und sozial unangepasstem oder selbstschädigendem Verhalten (niedrigeres Schulniveau). Die BPS gipfelt wahrscheinlich erst einige Jahre später in die psychosozial ausschlaggebende Dekompensation, wenn die Ausbildungszeit bereits abgeschlossen ist, aber die berufliche Etablierung noch aussteht. Viele Patienten mit BPS retten sich möglicherweise aus der Arbeitslosigkeit heraus in ausbildungsfremde Jobs, weil sie von ihrem selbstständigen Einkommen leben müssen und weil dies ihrem Wunsch nach Autonomie entspricht.

Eine starke Korrelation von BPS und Arbeitsunfähigkeit und damit Angewiesenheit auf staatliche Unterstützungszahlungen zeigt die Studie von Samsone et al. (2003).

Dennoch findet sich die BPS fast nie als Begründung für einen Rentenantrag. Dies ist nennenswert, weil für die BPS das krankheitsbedingte Ausscheiden aus Beruf oder Ausbildung negativ mit dem Wiedereintritt ins Berufsleben assoziiert wird. Eine entsprechende Führsorge für die Patienten mit BPS ist bisher weniger tiefgreifend. 
Die soziale Rehabilitation sollte in ihrer fördernden Bedeutung für die Genesung und Stabilität der Patienten erkannt und als präventive Chance genutzt werden.

\subsection{Einschränkungen der Untersuchung}

Bei der Interpretation der Ergebnisse müssen verschiedene Limitationen berücksichtigt werden. Zunächst ist darauf hinzuweisen, dass es sich um eine retrospektive Auswertung ohne Kontrollgruppe handelt. Sie erlaubte nicht, die Erfolge der Therapie zu beurteilen. Angaben über die Wirksamkeit einzelner Medikamente waren nur sehr selten dokumentiert und wurden hier auch nicht bewertet.

Bei der Patientenstichprobe handelte es sich um stationär behandelte, vermutlich schwerer kranke Patienten, so dass die Ergebnisse nicht ohne weiteres auf ambulante Patienten oder BPS-Patienten allgemein übertragbar sind. Berücksichtigt man allerdings die Tatsache, dass ein bedeutender Anteil der ambulanten BorderlinePatienten zumindest einmal psychiatrisch-stationär aufgenommen wurde (z.B. Swartz et al. 1990), ist eine Generalisierung auch auf ambulante Patienten, zumindest eingeschränkt, möglich. Problematisch ist allerdings, dass das Patientenkollektiv mit milden, moderaten und schweren Fällen sehr heterogen ist.

Die Patientenrekrutierung fand in einer psychiatrischen-psychotherapeutischen Klinik statt, in der hauptsächlich Kriseninterventionen stattfinden so dass prozentual der stationäre Psychotherapie-Anteil im Vergleich zu Stichproben anderer Studien geringer ausfällt. Angaben über die Aufenthalte im Allgemeinen sind daher eher auf die Akuttherapie zu beziehen.

Die hohe Frequenz an bestehenden psychiatrischen Komorbiditäten lässt es zunächst problematisch erscheinen, spezifische Rückschlüsse auf die BPS zu ziehen. Allerdings muss man den Großteil der erwähnten Komorbiditäten als Merkmal oder Folge der BPS sehen, so dass diese der BPS unterzuordnen sind. Unter diesem Gesichtspunkt können die in dieser Arbeit gemachten Aussagen, direkt auf die BPS bezogen werden und haben somit auch Konsequenzen bezüglich der Pharmakotherapie.

Bei der Bewertung der Veränderungen in Bezug auf die Sozialdemografie ist zu beachten, dass aufgrund des Untersuchungsdesigns keine Referenzgruppe ohne 
BPS-Diagnose parallel untersucht wurde. Damit blieb der Einfluss gesamtgesellschaftlicher Entwicklungen unberücksichtigt. 


\section{Zusammenfassung}

Krankheitsbedingte Defizite in der alltäglichen sozialen Interaktion sind dafür verantwortlich, dass Patienten mit Borderline-Persönlichkeitsstörungen (BPS) nicht ihren persönlichen Möglichkeiten entsprechend sozial adäquat integriert sind.

Obwohl die Versorgungsressourcen aufgrund der weitreichenden psychosozialen Beeinträchtigungen der betroffenen Patienten stark gefordert sind, findet die BPS in der Aufmerksamkeit von Strukturanalysen zur Versorgungsqualität bisher weniger Berücksichtigung als viele andere psychische Störungen.

Obwohl in der Therapie der BPS die Psychopharmakotherapie gegenüber der Psychotherapie nicht die vorrangige Maßnahme darstellt, findet sie in der Praxis dennoch umfangreich Anwendung.

In der vorliegenden Studie wurden im Zeitraum 2008-2012 87 Borderline-Patienten mit insgesamt 140 Aufenthalten analysiert. Retrospektiv erfolgte eine Auswertung aus den Krankenakten. Es zeigte sich, dass $94 \%$ der Patienten in ihrer stationären Behandlungsgeschichte Psychopharmaka bekamen. Im Zeitraum 1998-2004 waren es $87 \%$. Hierbei wurden keine signifikanten Unterschiede in der Häufigkeit einer Psychopharmakotherapie bei Kriseninterventions- und Psychotherapie-Aufenthalten gefunden. Es wurden Medikamente aus allen psychotropen Substanzklassen eingesetzt, am häufigsten wie auch im ersten Beobachtungsintervall jedoch Antidepressiva. In der ersten Gruppe waren es 73,2\%, in der vorliegenden Studie 64\% der Patienten, die bei der Aufnahme und während der stationären Behandlung schon Erfahrungen damit gemacht hatten. Auffallend war im Vergleich zu anderen Studien aber auch, dass etwas weniger Neuroleptika verordnet wurden (Erfahrung bei $40 \%$ der Patienten). Im Zeitraum 2008-2012 waren es 46,5\% der Patienten. Vergleicht man beide Zeitfenster bezüglich Hypnotika und Sedativa, so dominierte der erste Beobachtungsraum mit 45,1\%. Im zweiten Zeitintervall hatten 29\% der Patienten das Medikament bekommen. Als ungewöhnlich erwies sich, dass die Substanzen eingesetzt wurden, die nach empirischer Datenlage bei der BPS nicht primär empfohlen werden und teilweise sogar nachteilige Effekte erwiesen haben (z.B. Benzodiazepine).

Die Behandlung der BPS erfolgt syndromorientiert, daher wurde die Gabe der verschiedenen Substanzklassen nach Syndromen aufgeschlüsselt; so bekamen 
beispielsweise Patienten mit affektiven Symptomen gehäuft Antidepressiva und sich selbst verletzende Patienten vermehrt Neuroleptika.

Im Laufe eines stationären Aufenthaltes nahm die Anzahl der Medikamente um durchschnittlich 27\% zu, die Patienten gingen mit signifikant mehr Medikamenten als sie gekommen waren. Auch im Zeitverlauf im Vergleich zum Beobachtungszeitraum (1996-2004) kam es zu einer allgemeinen Zunahme der pro stationärem Aufenthalt verabreichten verschiedenen Medikamenten und zu einer signifikanten Zunahme der Häufigkeit einer Psychopharmakotherapie bei verkürzter Liegezeit.

Hohe Prozentzahlen an Komorbiditäten und die soziodemographischen Daten verdeutlichten die Schwere der Krankheit, die offensichtlich häufig einer gezielten Psychopharmakotherapie bedarf.

Bei den Borderline-Patienten fanden sich hohe Raten an Psychopharmakotherapie und intensiver Polypharmazie; eine Psychopharmakotherapie erfolgte in ebenso hohem Maße wie bei anderen psychiatrischen Krankheitsbildern. Folglich besteht ein Bedarf an zukünftiger Forschung zu wirksamen Medikamenten bei der BorderlineStörung. 


\title{
6 Anhang
}

\subsection{Abbildungs- und Tabellenverzeichnis}

\author{
Abbildungsverzeichnis
}

Abbildung 1 Typischer Ablauf von Spannungsaufbau und Spannungslösung im Kontext von selbstschädigenden Verhaltensweisen bei der BPS...... 9

Abbildung 2 Behandlungsalgorithmus bei BPS bei verschiedenen

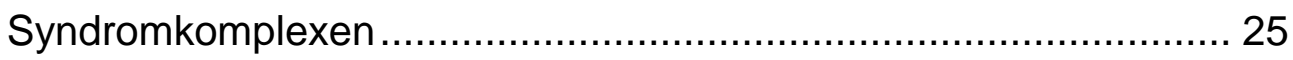

Abbildung $3 \quad$ Komorbiditäten (ärztlich gestellte Diagnosen) …............................ 35

Abbildung 4 Komorbiditäten-Psychische und Verhaltensstörungen durch psychotrope Substanzen ........................................................... 36

Abbildung $5 \quad$ Suizidversuche in der Anamnese ................................................. 37

Abbildung 6 Dokumentation der einzelnen diagnostischen Kriterien nach ICD-10 (WHO 1993) 38

Abbildung 7 Dokumentation der einzelnen diagnostischen Kriterien nach DSM-IV (APA 1994) 39

Abbildung 8 Aufnahmeanlass für den stationären Aufenthalt. 40

Abbildung 9 Dauer der stationären Behandlung bei verschiedenen Behandlungsmodi

Abbildung 10 Einnahme von Psychopharmaka bei Aufnahme, während stationärer Behandlung und als Reserve.

Abbildung 11 Gabe verschiedener Psychopharmaka-Hauptgruppen bei Aufnahme

Abbildung 12 Gabe verschiedener Psychopharmakahauptgruppen bei

Entlassungen 43

Abbildung 13 Einnahme von Neuroleptika bei Aufnahme und während stationärer Behandlung 44

Abbildung 14 Einnahme von Neuroleptika bei Entlassung; 45

Abbildung 15 Einnahme von Antidepressiva bei Aufnahme und während stationärer Behandlung 45

Abbildung 16 Gabe von Antidepressiva bei Entlassung 46 


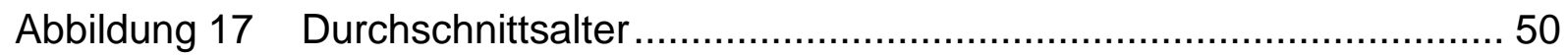

Abbildung 18 Dauer der Aufenthalte ................................................................ 51

Abbildung 19 Vergleich Ehe prozentual......................................................... 54

Abbildung 20 Vergleich Ehe statistisch ...................................................... 54

Abbildung 21 Vergleich Partnerschaft prozentual............................................ 55

Abbildung 22 Vergleich Partnerschaft statistisch............................................. 55

Abbildung 23 Vergleich Partnerwechsel prozentual ......................................... 56

Abbildung 24 Vergleich Partnerwechsel statistisch .......................................... 56

Abbildung 25 Vergleich sexuelle Ausrichtung prozentual .................................. 57

Abbildung 26 Vergleich sexuelle Ausrichtung statistisch ................................... 57

Abbildung 27 Vergleich Wohnsituation prozentual .......................................... 58

Abbildung 28 Vergleich Wohnsituation statistisch ........................................... 59

Abbildung 29 Vergleich Tätigkeit prozentual .................................................. 60

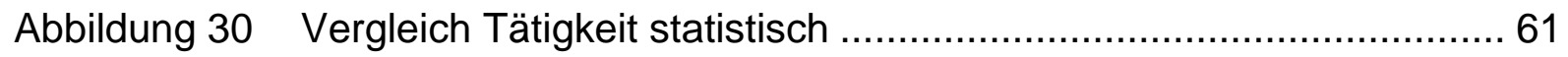

Abbildung 31 Vergleich schulischer Abschluss prozentual................................. 62

Abbildung 32 Vergleich schulischer Abschluss statistisch.................................. 62

Abbildung 33 Vergleich beruflicher Abschluss prozentual ...................................63

Abbildung 34 Vergleich beruflicher Abschluss statistisch ...................................63

Abbildung 35 Vergleich Verwandte mit psychischen Diagnosen prozentual ......... 64

Abbildung 36 Vergleich Verwandte mit psychischen Diagnosen statistisch .......... 64

Abbildung 37 Vergleich Verwandte mit Hinweis auf BPS prozentual ................... 65

Abbildung 38 Vergleich Verwandte mit Hinweis auf BPS statistisch ..................... 65

Abbildung 39 Vergleich Komorbiditäten prozentual ......................................... 66

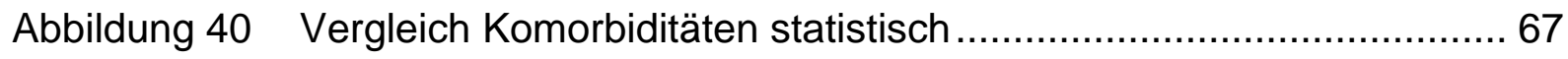

Abbildung 41 Vergleich Komorbiditäten - Psychische und Verhaltensstörungen durch psychotrope Substanzen prozentual.................................... 68

Abbildung 42 Vergleich Komorbiditäten - Psychische und Verhaltensstörungen durch psychotrope Substanzen statistisch..................................... 69

Abbildung 43 Vergleich Suizidversuche in der Anamnese prozentual.................. 70

Abbildung 44 Vergleich Suizidversuche in der Anamnese statistisch................... 70

Abbildung 45 Vergleich der einzelnen diagnostischen Kriterien nach ICD-10 (WHO

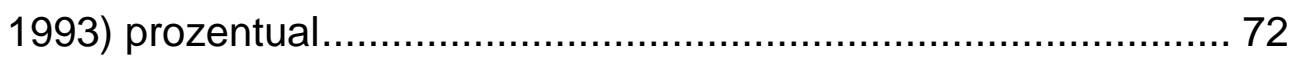

Abbildung 46 Vergleich der einzelnen diagnostischen Kriterien nach ICD-10 (WHO

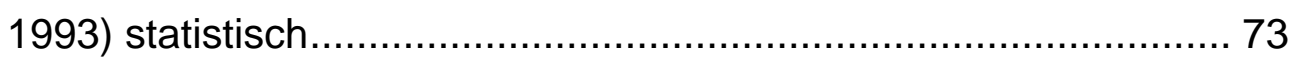


Abbildung 47 Vergleich der diagnostischen Kriterien nach DSM-IV (APA 1994) prozentual

Abbildung 48 Vergleich der diagnostischen Kriterien nach DSM-IV (APA 1994) statistisch.....

Abbildung 49 Vergleich der Einnahme von Neuroleptika bei Entlassung prozentual

Abbildung 50 Vergleich der Einnahme von Neuroleptika bei Entlassung statistisch.

Abbildung 51 Vergleich Psychopharmakahauptgruppen bei Entlassung prozentual

Abbildung 52 Vergleich Psychopharmakahauptgruppen bei Entlassung statistisch.

Abbildung 53 Vergleich Patienten, die bei der Entlassung Antidepressiva bekamen prozentual 80

Abbildung 54 Vergleich Patienten, die bei der Entlassung Antidepressiva bekamen statistisch 80

\section{Tabellenverzeichnis}

Tabelle 1 Übersicht über die verschiedenen Persönlichkeitsstörungen in der ICD-10

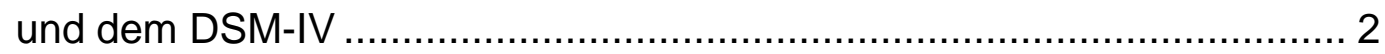

Tabelle 2 Übersicht über bisherige Medikamentenstudien ................................. 20

Tabelle 3 Medikamentenwirkungen bei der Borderline-Persönlichkeitsstörung...... 23

Tabelle 4 Soziodemographie ............................................................... 32

Tabelle 5 Verhältnis von Abhängigkeit und Abusus bei verschiedenen

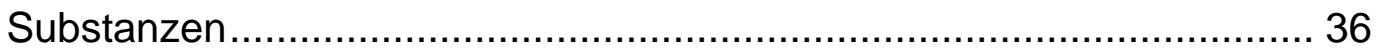

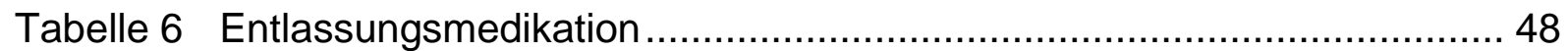

Tabelle 7 Vergleich Soziodemographie zwischen 2008 - 2012 und 1996 - 2004 ... 51 


\subsection{Das Erhebungsinstrument - der Fragebogen}

Auf den folgenden Seiten findet sich der Fragebogen, mit dem die Daten für die vorliegende Arbeit erhoben wurden.

Die Seiten 102 bis 109 wurden je nach Anzahl der stationären Aufenthalte des jeweiligen Patienten dem Fragebogen mehrfach hinzugefügt.

Die Auswertung der Erhebung geschah in einer anderen Reihenfolge als im Fragebogen vorgegeben. Die Einteilung der Medikamente in Substanzgruppen wurde entsprechend der „Roten Liste“ überarbeitet. 


\section{Borderline-Studie}

Datum:

Patient:

Geb. Dat:
wO m O

Aktennummer:

Psychiatrische Diagnosen (Gesamtzeitraum in zeitlicher Folge der Diagnosestellung):

\begin{tabular}{|l|l|}
\hline 1. & \\
\hline 2. & \\
\hline 3. & \\
\hline 4. & \\
\hline 5. & \\
\hline 6. & \\
\hline 7. & \\
\hline 8. & \\
\hline 9. & \\
\hline 10. & \\
\hline
\end{tabular}

Substanzabhängigkeiten und -abusus

\begin{tabular}{|l|l|l|l|l|}
\hline & F10 & F11 & F12 & F13 \\
\hline Abusus & & & & \\
\hline Abhängigkeit & & & & \\
\hline
\end{tabular}

Andere Abhängigkeiten /Abusus

\section{Essstörungen $O$}

Anzahl an Suizidversuchen: 


\section{Stationäre Behandlungen}

Anzahl gesamt:



KI: Krisenintervention; PT: Psychotherapie; SB: Suchtbehandlung; KJP: Kinder- und Jugendpsychiatrie 


\section{Dokumentation diagnostischer Kriterien nach DSM-IV}

\begin{tabular}{|l|l|l|}
\hline & Symptombereich & wird beschrieben \\
\hline 1) & $\begin{array}{l}\text { Impulsivität bei mindestens zwei potentiell selbst schädigenden } \\
\text { Aktivitäten: z.B. Drogenabusus, Alkoholabusus, Glücksspiel, } \\
\text { Ladendiebstahl }\end{array}$ & $\begin{array}{l}\text { Unbeständige und intensive zwischenmenschliche Beziehungen, } \\
\text { mit einem Wechsel zwischen den Extremen der Idealisierung und } \\
\text { Abwertung }\end{array}$ \\
\hline 2) & $\begin{array}{l}\text { Übermäßige, starke Wut oder Unfähigkeit, die Wut zu kontrollieren } \\
\text { 3) }\end{array} \quad \begin{array}{l}\text { Identitätsstörung: deutliches und dauerhaft bestehendes } \\
\text { unbeständiges Selbstbild }\end{array}$ & \\
\hline 4$)$ & $\begin{array}{l}\text { Affektive Instabilität mit starken Stimmungsschwankungen } \\
\text { Verzweifeltes Bemühen, ein reales oder vorgestelltes }\end{array}$ & \\
\hline 5) & $\begin{array}{l}\text { Häufig Suiziddrohungen, -andeutungen, -versuche oder } \\
\text { Selbstverletzungen }\end{array}$ & \\
\hline 6) & Chronische Leeregefühle & \\
\hline 8$)$ & $\begin{array}{l}\text { Vorübergehende, stressbedingte Wahnvorstellungen oder schwere } \\
\text { dissoziative Symptome }\end{array}$ & \\
\hline 9$)$ &
\end{tabular}

Gesamtzahl:

Kriterien gesamt nach DSM-IV erfüllt (mind. 5 von 9): ja $\mathrm{n}$ nein $\mathrm{O}$

\section{Dokumentation diagnostischer Kriterien nach ICD-10}

\begin{tabular}{|c|c|c|}
\hline & Symptombereich & wird beschrieben \\
\hline 1) & $\begin{array}{l}\text { Deutliche Tendenz, unerwartet und ohne Berücksichtigung der } \\
\text { Konsequenzen zu handeln }\end{array}$ & \\
\hline 2) & $\begin{array}{l}\text { Deutliche Tendenz zu Streitereien und Konflikten mit anderen, } \\
\text { vor allem dann, wenn impulsive Handlungen unterbunden und } \\
\text { getadelt werden }\end{array}$ & \\
\hline 3) & $\begin{array}{l}\text { Neigung zu Ausbrüchen von Wut oder Gewalt mit Unfähigkeit zur } \\
\text { Kontrolle explosiblen Verhaltens }\end{array}$ & \\
\hline 4) & $\begin{array}{l}\text { Schwierigkeiten in der Beibehaltung von Handlungen, die nicht } \\
\text { unmittelbar belohnt werden }\end{array}$ & \\
\hline 5) & Unberechenbare und unbeständige Stimmung & \\
\hline a) & $\begin{array}{l}\text { Störung und Unsicherheit bezüglich Selbstbild, Zielen und innerer } \\
\text { Präferenzen (einschließlich sexueller) }\end{array}$ & \\
\hline b) & $\begin{array}{l}\text { Neigung sich auf intensive aber instabile Beziehungen } \\
\text { einzulassen, oft mit der Folge von emotionalen Krisen }\end{array}$ & \\
\hline c) & Übertriebene Bemühungen, das Verlassenwerden zu vermeiden & \\
\hline d) & Wiederholt Drohungen oder Handlungen mit Selbstschädigung & \\
\hline e) & Anhaltende Gefühle von Leere & \\
\hline
\end{tabular}

Gesamtzahl:

Kriterien gesamt nach ICD-10 erfüllt (mind. 3 aus 1-5 + mind. 2 aus a-e): ja Onein $O$ 


\section{Soziodemographie}

\section{Partnerschaft}

Ehe:

eruierbar $\mathrm{O}$

ja $\mathrm{O}$ nein $\mathrm{O}$ geschieden $\mathrm{O}$

nicht

Partnerschaft: $\quad$ ja $\mathrm{O}$ nein $\mathrm{O}$ nicht eruierbar $\mathrm{O}$

Häufiger Partnerwechsel: ja $O$ nein $O$ nicht eruierbar $O$

Sexuelle Ausrichtung: homosexuell Oheterosexuell O

bisexuell $\quad \mathrm{O}$ nicht eruierbar $\mathrm{O}$

2. Wohnsituation

\begin{tabular}{|l|l|l|l|l|l|l|l|l|l|}
\hline allein & Mit Partner & & Im Heim & $\begin{array}{l}\text { Kein fester } \\
\text { Wohnsitz }\end{array}$ & Andere: & \\
\hline Bei Eltern & $\begin{array}{l}\text { Betreutes } \\
\text { Wohnen }\end{array}$ & $\begin{array}{l}\text { Bei } \\
\text { Pflegeeltern }\end{array}$ & $\begin{array}{l}\text { Stark } \\
\text { wechselnd }\end{array}$ & Nicht eruierbar & \\
\hline
\end{tabular}

3. Familie

- Verwandte 1.Grades: -Psychiatrische Diagnosen O

\section{Abschlüsse und Tätigkeit}

- Schulischer Abschluss:

\begin{tabular}{|l|l|l|l|l|l|l|}
\hline $\begin{array}{l}\text { Kein } \\
\text { Abschluss }\end{array}$ & $\begin{array}{l}\text { Sonderschulabschl } \\
\text { uss }\end{array}$ & $\begin{array}{l}\text { Hauptschulabschlu } \\
\text { ss }\end{array}$ & Realschulabschluss & \\
\hline Abitur & Noch in Ausbildung & Nicht eruierbar & & & \\
\hline
\end{tabular}

- Beruflicher Abschluss:

\begin{tabular}{|l|l|l|l|l|l|l|l|}
\hline $\begin{array}{l}\text { Kein } \\
\text { Abschluss }\end{array}$ & $\begin{array}{l}\text { Abgeschlossene } \\
\text { Ausbildung }\end{array}$ & $\begin{array}{l}\text { Abgeschlossenes } \\
\text { Studium }\end{array}$ & $\begin{array}{l}\text { Noch in } \\
\text { Ausbildung }\end{array}$ & Nicht eruierbar & \\
\hline
\end{tabular}

- Ausgeübte Tätigkeit:

\begin{tabular}{|l|l|l|l|l|l|l|l|}
\hline Schüler & $\begin{array}{l}\text { Auszubildende } \\
r\end{array}$ & Student & $\begin{array}{l}\text { In } \\
\text { Umschulung }\end{array}$ & $\begin{array}{l}\text { Bm gelernten } \\
\text { Beruf }\end{array}$ & Arbeitsunfähig & Erwerbsunfähig & \\
\hline $\begin{array}{l}\text { In } \\
\text { Angelernter }\end{array}$ & Arbeitslos & $\begin{array}{l}\text { Häufig } \\
\text { wechselnder } \\
\text { Arbeitsplatz }\end{array}$ & Maßregelvollzug & $\begin{array}{l}\text { Nicht } \\
\text { eruierbar }\end{array}$ & & \\
\hline In Rente & Hausfrau & Maßrar & & & \\
\hline
\end{tabular}


Stationäre Behandlung Nr.:_in:

von: bis:

- Aufnahmeanlass

\begin{tabular}{|l|l|}
\hline Suizidversuch & \\
\hline Parasuizidale Handlung & \\
\hline Suizidalität (angedroht) & \\
\hline Selbstschädigung & \\
\hline Intoxikation (nicht suizidal) & \\
\hline Angststörung & \\
\hline Affektive Symptome & \\
\hline Familiäre Konflikte & \\
\hline Arbeitsplatzprobleme/ -verlust & \\
\hline Ohne Obdach & \\
\hline Partnerschaftskonflikt /Trennung & \\
\hline Polizeiliche Vorstellung / Straftat & \\
\hline Entgiftungswunsch & \\
\hline Geplante Psychotherapie & \\
\hline Nicht eruierbar & \\
\hline Sonstige: & \\
\hline
\end{tabular}

- Syndromkomplex

\begin{tabular}{|c|c|c|c|}
\hline affektiv & impulsiv & psychotisch & nicht eruierbar \\
\hline
\end{tabular}

\section{- Suizidversuche, Parasuizidale Handlungen und Selbstverletzungen}

\begin{tabular}{|l|l|l|l|l|}
\hline & Im Vorfeld & Währ. stat. Behandl. & $\begin{array}{l}\text { Nicht } \\
\text { eruierbar }\end{array}$ & Nein \\
\hline Suizidversuche & & & & \\
\hline $\begin{array}{l}\text { Parasuizidale Handlungen oder fragliche } \\
\text { Suizidversuche }\end{array}$ & & & & \\
\hline Selbstverletzungen & & & & \\
\hline
\end{tabular}

- Wer betreute oder behandelte den Patienten vor der Aufnahme? (Mehrfachnennungen möglich)

\begin{tabular}{|l|l|l|l|l|l|l|l|}
\hline Andere Klinik & Tagesklinik & $\begin{array}{l}\text { Niedergelassener } \\
\text { Psychiater }\end{array}$ & Hausarzt & $\begin{array}{l}\text { Nicht } \\
\text { eruierbar }\end{array}$ & \\
\hline Psychotherapeut & Sozialdienst & Poliklinik & Keiner & & \\
\hline
\end{tabular}


- Wer betreut oder behandelt den Patienten weiter? (Mehrfachnennungen möglich)

\begin{tabular}{|l|l|l|l|l|l|l|l|l|}
\hline Andere Klinik & Tagesklinik & $\begin{array}{l}\text { Niedergelassener } \\
\text { Psychiater }\end{array}$ & Hausarzt & $\begin{array}{l}\text { Nicht } \\
\text { eruierbar }\end{array}$ & \\
\hline Psychotherapeut & Sozialdienst & & Poliklinik & & Keiner & & \\
\hline
\end{tabular}

\section{- Entlassungsmodus}

\begin{tabular}{|l|l|l|l|l|}
\hline regulär & Gegen ärztlichen Rat & $\begin{array}{l}\text { Aus disziplinarischen } \\
\text { Gründen }\end{array}$ & Nicht eruierbar \\
\hline $\begin{array}{l}\text { Verlegung auf andere } \\
\text { psychiatrische Station }\end{array}$ & $\begin{array}{l}\text { Verlegung auf andere nicht- } \\
\text { psychiatrische Station }\end{array}$ & & \\
\hline
\end{tabular}

\section{- Weiteres}

\begin{tabular}{|l|l|l|l|}
\hline & Ja & Nein & $\begin{array}{c}\text { Nicht } \\
\text { eruierbar }\end{array}$ \\
\hline Diagnose F 60.3 & & & \\
\hline Unterbringung nach PsychKG & & & \\
\hline Maßregelvollzug & & & \\
\hline Gesetzliche Betreuung eingerichtet oder beantragt & & & \\
\hline Arbeitsfähigkeit & & & \\
\hline
\end{tabular}

- Psychopharmakotherapie

\begin{tabular}{|c|c|c|c|c|}
\hline \multirow[t]{2}{*}{ Psychopharmakon } & \multirow{2}{*}{$\begin{array}{l}\text { Vormedikation: } \\
\text { Dosis (mg) }\end{array}$} & \multicolumn{2}{|c|}{ Währendmedikation: } & \multirow{2}{*}{$\begin{array}{l}\text { Entlassungsmedikation: } \\
\text { Dosis (mg) }\end{array}$} \\
\hline & & Dosis (mg) & Tage & \\
\hline \multicolumn{5}{|l|}{ Neuroleptika } \\
\hline \multicolumn{5}{|l|}{ Amisulprid (Solian) } \\
\hline \multicolumn{5}{|l|}{ Chlorprothixen (Truxal) } \\
\hline \multicolumn{5}{|l|}{ Clozapin (Leponex) } \\
\hline \multirow{2}{*}{\multicolumn{5}{|c|}{ Flupentixol (Fluanxol) }} \\
\hline & & & & \\
\hline \multicolumn{5}{|l|}{ Fluspirilen (Imap) } \\
\hline \multicolumn{5}{|l|}{ Haloperidol (Haldol) } \\
\hline \multicolumn{5}{|c|}{ Levomepromazin (Neurocil) } \\
\hline \multicolumn{5}{|c|}{ Melperon (Eunerpan) } \\
\hline \multicolumn{5}{|l|}{ Olanzapin (Zyprexa) } \\
\hline \multicolumn{5}{|l|}{ Perazin (Taxilan) } \\
\hline \multicolumn{5}{|l|}{ Perphenazin (Decetan) } \\
\hline \multicolumn{5}{|l|}{ Pimozid (Orap) } \\
\hline \multicolumn{5}{|l|}{ Pipamperon (Dipiperon) } \\
\hline \multicolumn{5}{|l|}{ Promazin (Protactyl) } \\
\hline \multicolumn{5}{|l|}{ Promethazin (Atosil, } \\
\hline \multicolumn{5}{|l|}{ Prothipendyl (Dominal) } \\
\hline \multicolumn{5}{|l|}{ Quetiapin (Seroquel) } \\
\hline \multirow{2}{*}{\multicolumn{5}{|c|}{ Risperidon (Risperdal) }} \\
\hline & & & & \\
\hline \multicolumn{5}{|l|}{ Thioridazin (Melleril) } \\
\hline \multirow{2}{*}{\multicolumn{5}{|c|}{ Zotepin (Nipolept) }} \\
\hline & & & & \\
\hline Antidepressiva & & & & \\
\hline
\end{tabular}




\begin{tabular}{|c|c|c|c|c|}
\hline \multirow[t]{2}{*}{ Psychopharmakon } & \multirow{2}{*}{$\begin{array}{l}\text { Vormedikation: } \\
\text { Dosis (mg) }\end{array}$} & \multicolumn{2}{|c|}{ Währendmedikation: } & \multirow{2}{*}{$\begin{array}{l}\text { Entlassungsmedikation: } \\
\text { Dosis }(\mathrm{mg})\end{array}$} \\
\hline & & Dosis (mg) & Tage & \\
\hline \multicolumn{5}{|l|}{ Alprazolam (Tafil) } \\
\hline \multicolumn{5}{|l|}{ Amitriptylin (Saroten; } \\
\hline \multicolumn{5}{|l|}{ Citalopram (Cipramil; Cilex) } \\
\hline \multicolumn{5}{|l|}{ Clomipramin (Anafranil) } \\
\hline \multirow{2}{*}{\multicolumn{5}{|c|}{$\begin{array}{l}\text { Desipramin (Pertrofan; } \\
\text { Dibenzepin (Noveril) }\end{array}$}} \\
\hline & & & & \\
\hline \multicolumn{5}{|l|}{$\begin{array}{l}\text { Dibenzepin (Noveril) } \\
\text { Dosulepin (Idom) }\end{array}$} \\
\hline \multicolumn{5}{|l|}{ Doxepin (Aponal) } \\
\hline \multicolumn{5}{|l|}{ Fluoxetin (Fluctin) } \\
\hline \multicolumn{5}{|l|}{ Fluvoxamin (Fevarin) } \\
\hline \multicolumn{5}{|l|}{ Imipramin (Tofranil) } \\
\hline \multirow{2}{*}{\multicolumn{5}{|c|}{$\begin{array}{l}\text { Lofepraminhydrochlorid(Ga } \\
\text { Maprotilin (Ludiomil: }\end{array}$}} \\
\hline & & & & \\
\hline \multicolumn{5}{|l|}{ Mianserin (Tolvin; Prisma) } \\
\hline \multicolumn{5}{|l|}{ Mirtazapin (Remergil) } \\
\hline Moclobemid (Aurorix) & & & & \\
\hline Nefazodon (Nefadar) & & & & \\
\hline Nortriptylin (Noveril) & & & & \\
\hline Opipramol (Insidon) & & & & \\
\hline Paroxetin (Tagonis, & & & & \\
\hline Reboxetin (Edronax) & & & & \\
\hline Sertralin (Zoloft) & & & & \\
\hline Sulpirid (Dogmatil; Meresa) & & & & \\
\hline Tranylcypromin (Jatrosom) & & & & \\
\hline Trazodon (Thombran) & & & & \\
\hline Trimipramin (Stangyl; & & & & \\
\hline Venlafaxin (Trevilor) & & & & \\
\hline Tranquillantia/ & & & & \\
\hline Alprazolam (Tafil; Xanax) & & & & \\
\hline Bromazepam & & & & \\
\hline Clomethiazol (Distraneurin) & & & & \\
\hline Clonazepam (Rivotril) & & & & \\
\hline Diazepam (Valium) & & & & \\
\hline Dikaliumclorazepat & & & & \\
\hline Flurazepam (Dalmadorm) & & & & \\
\hline Lorazepam (Tavor) & & & & \\
\hline Lormetazepam (Noctamid) & & & & \\
\hline Midazolam ( Dormicum) & & & & \\
\hline Nordazepam (Tranxilium & & & & \\
\hline Oxazepam (Adumbran) & & & & \\
\hline Temazepam (Planum; & & & & \\
\hline Triazolam (Halcion) & & & & \\
\hline Zaleplon (Sonata) & & & & \\
\hline Zolpidem (Stilnox) & & & & \\
\hline Zolpidemtartrat (Bikalm) & & & & \\
\hline Zoplicon (Ximovan) & & & & \\
\hline Mood Stabilizer & & & & \\
\hline Carbamazepin (Tegretal; & & & & \\
\hline Gabapentin (Neurontin) & & & & \\
\hline Lithium (Hypnorex; & & & & \\
\hline Valproat (Ergenyl; Orfiril & & & & \\
\hline Andere & & & & \\
\hline Chloralhydrat (Chloraldurat) & & & & \\
\hline Naltrexon (Nemexin) & & & & \\
\hline Levomethadon (Polamidon) & & & & \\
\hline Baldrian (Valdispert) & & & & \\
\hline Johanniskraut (Sedariston) & & & & \\
\hline
\end{tabular}


- Reservemedikation

\begin{tabular}{|l|l|l|l|l|l|}
\hline Wirkstoff & Höchstdosis/Tag & $\begin{array}{l}\text { Angesetzter } \\
\text { Zeitraum }\end{array}$ & Gabetage & Häufigkeit & Begründung \\
\hline & & & & & \\
\hline & & & & & \\
\hline & & & & & \\
\hline & & & & & \\
\hline & & & & & \\
\hline
\end{tabular}

- Dokumentation über Wirksamkeit der Pharmaka Ja O Nein O

- Behandlungsmodus

\begin{tabular}{|l|l|l|l|l|l|l|l|l|}
\hline Krisenintervention & Psychotherapie & Suchtbehandlung & $\begin{array}{l}\text { Kinder- } \\
\text { /Jugendpsychiatrie }\end{array}$ & $\begin{array}{l}\text { Nicht } \\
\text { eruierbar }\end{array}$ & \\
\hline
\end{tabular}

\section{- Durchgeführte Therapien}

○ Nicht- Strukturierte Therapie

\begin{tabular}{|l|l|l|l|l|l|l|l|}
\hline $\begin{array}{l}\text { Einzelgesprä } \\
\text { che }\end{array}$ & Gruppentherapie & & Ergotherapie & Physiotherapie & \\
\hline Entspannung & $\begin{array}{l}\text { Andere: } \\
-\end{array}$ & Keine & Nicht eruierbar & \\
\hline
\end{tabular}

- Strukturierte Psychotherapie

\begin{tabular}{|l|l|l|l|l|l|l|l|}
\hline $\begin{array}{l}\text { Psychoanalytisch/ } \\
\text { Tiefenpsychologisch }\end{array}$ & $\begin{array}{l}\text { Verhaltenstherapie } \\
\text { / DBT }\end{array}$ & andere & Nicht eruierbar & \\
\hline
\end{tabular}




\section{Literaturverzeichnis}

Andrulonis PA, Glueck BC, Stroebel CF, Vogel NG (1982): Borderline personality subcategories. J Nerv Ment Dis 170(11), 670-679

APA (American Psychiatric Association): Diagnostic and Statistical Manual of Mental Disorders; American Psychiatric Association, Washington DC 1980.

APA (American Psychiatric Association): Diagnostic and Statistical Manual of Mental Disorders; American Psychiatric Association, Washington DC 1994.

APA (American Psychiatric Association) (2001): Practice guideline for the treatment of patients with borderline personality disorder. Am J Psychiatry 158, 1-54

Apter A, Plutchik R, van Praag H.M (1993): Anxiety, impulsivity and depressed mood in relation to suicidal and violent behavior. Acta Psychiatrica Scandinavia $\underline{87}, 1-5$

Bandelow B,Schmal C,Falkai P,Wedekind D (2010): Borderline personality disorder: a dysregulation of the endogenous opioid system? Psychol Rev. 117, 623-636

Baron M, Gruen R, Asnis L, Lord S (1985): Familial transmission of schizotypal and borderline personality disorders. American Journal of Psychiatry 142, 927-934

Bateman A, Fonagy F (1999): Effectiveness of partial hospitalization in the treatment of borderline personality disorder: a randomized controlled trial. Am J Psychiatry 156, 1563-1569

Bateman A, Fonagy $F$ (2001): Treatment of borderline personality disorder with psychoanalytically oriented partial hospitalization: an 18-month follow-up. Am J Psychiatry $\underline{158}, 36-42$

Bellino S, Paradiso E, Bogetto F (2008): Efficacy and tolerability of aripiprazole augmentation in sertraline-resistant patients with borderline personality disorder. Psychiatry Res. 161, 206-212

Bellino S, Rinaldi C, Bozzatello P, Bogetto F (2011): Pharmacotherapy of borderline personality disorder: a systematic review for publication purpose. Curr Med Chem. $\underline{18}$, 3322-3329

Bellino S, Patria L, Paradiso E, Di Lorenzo R, Zanon, C, Zizza M, Bogetto F (2005): Major Depression in Patients With Borderline Personality Disorder: A Clinical Investigation. Canadian Journal of Psychiatry, $\underline{50}$, 234-238

Bender DS, Dolan RT, Skodol AE, Sanislow CA, Dyck IR, McGlashan TH, Shea MT, Zanarini MC, Oldham JM, Gunderson JG (2001): Treatment utilization by patients with personality disorders. Am J Psychiatry 158, 295-302 
Benedetti F, Sforzini L, Colombo C, Maffei C, Smeraldi E (1998): Low-dose clozapine in acute and continuation treatment of severe borderline personality disorder. $\mathrm{J}$ Clin Psychiatry $\underline{59}$, 103-107

Bick PA, Hannah AL (1986): Intramuscular lorazepam to restrain violent patients. Lancet $\underline{1}, 206$

Binks CA, Fenton M, McCarty L, Lee T, Adams CE, Duggan C (2006): Psychological therapies for people with borderline personality disorder (review). Cochrane database of systematic reviews 1

Bogenschutz MP, Nurnberg GH (2004): Olanzapine versus placebo in the treatment of borderline personality disorder. J Clin Psychiatry $\underline{65}, 104-109$

Bohus M: Borderline-Störung. Hogrefe, Göttingen 2002

Bohus M: Borderlinepersönlichkeitsstörungen. In: Lehrbuch der Verhaltenstherapie. Hrsg. von Margraf J. Band 2, 2. Auflage; Springer-Verlag, Heidelberg 1999, 413-437

Bohus M, Berger M (1996): Die Dialektisch-Behaviorale Psychotherapie nach M Linehan. Ein neues Konzept zur Behandlung von BorderlinePersönlichkeitsstörungen. Nervenarzt $\underline{67}, 911-23$

Bohus M, Remmel A (2004): Zum Umgang mit Suizidalität in der Borderline-Therapie. Persönlichkeitsstörungen $\underline{8}, 11-16$

Bohus M, Schmahl C (2006): Psychopathologie und Therapie der BorderlinePersönlichkeitsstörung. Dtsch Arztebl $\underline{49}$, 3345-3351

Bohus M, Stieglitz RD, Fiedler P, Berger M: Persönlichkeitsstörungen. In: Psychiatrie und Psychotherapie. Hrsg. von Berger M; Urban und Schwarzenberg Verlag, München, Wien, Baltimore 1999, 771-845

Bohus M, Landwehrmeyer BG, Stiglmeyr CE, Limberger MF, Bohme R, Schmahl CG (1999): Naltrexone in the treatment of dissociative symptoms in patient with borderline personality disorder: an open-label trial. J Clin Psychiatry $\underline{60}$, 598-603.

Bohus M, Limberger M, Ebner U, Glocker FX, Schwarz,B, Wernz M, Lie K (2000): Pain perception during self-reported distress and calmness in patients with borderline personality disorder and self-mutilating behavior. Psychiatry Research 95, 251-260

Bremner, JD (1999): Does stress damage the brain? Biological Psychiatry $\underline{45}, 797-$ 805

Brodsky BS, Malone K, Ellis SP, Dulit RA, Mann JJ (1997): Characteristics of borderline personality disorders associated with suicidal behavior. Am Journal of Psychiatry $\underline{154}, 1715-1719$

Bronisch T (2001): Neurobiologie der Persönlichkeitsstörungen mit dem Schwerpunkt der Borderline Persönlichkeitsstörung. Psychotherapie $\underline{6}$, 233-246 
Bronisch T: Suizidalität. In: Psychiatrie und Psychotherapie. Hrsg. v. Möller HJ, Laux G, Kampfhammer HP. Springer, Berlin 2003

Buchheim A, Strauss B, Kächele H (2002): Die differentielle Relevanz der Bindungsklassifikationen für psychische Störungen: Zum Stand der Forschung bei Angststörungen, Depression und Borderline-Persönlichkeitsstörung. Psychother Psych Med $\underline{52}$, 128-133

Chengappa KN, Ebeling T, Kang JS, Levine J, Parepally H (1999): Clozapine reduces severe self-mutilation and aggression in psychotic patients with borderline personality disorder. J Clin Psychiatry $\underline{60}$, 477-84

Clarkin J, Foelsch PA, Levy KN, Hull JW, Delaney JC, Kernberg OF (2001): The development of a psychodynamic treatment for patients with borderline personality disorder: a preliminary study of behavioral change. Journal of Personality Disorders $\underline{15}, 487-495$

Coccaro EF, Kavoussi R.J (1997): Fluoxetine and impulsive aggressive behaviour in personality-disordered subjects. Archives of General Psychiatry $\underline{54}, 1081-1088$

Cowdry RW, Gardner DL (1988): Pharmacotherapy of borderline personality disorder: Alprazolam, carbamazepine, trifluoperazine and tranylcypromine. Arch Gen Psychiatry $\underline{45}, 111-119$

Davids E, Gastpar M (2005): Attention deficit hyperactivity disorder and borderline personality disorder. Progress in Neuro-Psychopharmacology and Biological Psychiatry $\underline{29}$, 865-877

De La Fuente JM, Lotstra F (1994): A trial of carbamazepine in borderline personality disorder. Eur Neuropsychopharmacol $\underline{4}, 479-486$

Dell'Osso B, Berlin HA, Serati M, Altamura AC (2010): Neuropsychobiological aspects, comorbidity patterns and dimensional models in borderline personality disorder. Neuropsychobiology $\underline{61}, 169-179$

Díaz-Marsá M, Galian M, Montes A, Fernández R, Arza R, López-lbor J, Carrasco J (2008): Long-acting injectable risperidone in treatment resistant borderline personality disorder. A small series report. Actas Esp Psiquiatr 36,70-74

Dilling $\mathrm{H}$, Mombour W, Schmidt MH: ICD-10, Kapitel v (F), Klinisch-diagnostische Leitlinien. In: Dilling H, Mombour W, Schmidt MH (Hrsg): Internationale Klassifikation psychischer Störungen. Huber, Bern 2005.

Dowson JH, McLean A, Bazanis E, Toone B, Young S, Robbins TW, Sahakian B (2004): The specificity of clinical characteristics in adults with attentiondeficit/hyperactivity disorder: a comparison with patients with borderline personality disorder. European Psychiatry 19, 72-78

Driessen M, Beblo T, Reddemann L, Rau H, Lange W, Silva A, Berea RC, Wulff $H$, Ratzka S (2002): Ist die Borderline-Persönlichkeitssörrung eine komplexe posttraumatische Störung? Nervenarzt $\underline{73}$, 820-829 
Dulz B, Schneider A: Borderline-Störungen. 2. Auflage; Schattauer Verlag, Stuttgart 1996

Faltus FJ (1984): The positive effect of alprazolam in the treatment of three patients with borderline personality disorder. Am J Psychiatry 141, 802-803

Fonagy P, Gergely G, Jurist E, Target M, Leigh T, Steele M, Steele H, Kennedy R, Mattoon G, Target M, Gerber A (1996): The relation of attachment status, psychiatric classification, and response to psychotherapy. Journal of Consulting \& Clinical Psychology $\underline{64}, 22-31$

Frankenburg FR, Zanarini MC (1993): Clozapine treatment of borderline patients: A preliminary study. Compr Psychiatry $\underline{34}$, 402-405

Frankenburg FR, Zanarini MC (2002): Divalproex sodium treatment of women with borderline personality disorder and bipolar II disorder: a double-blind placebocontrolled pilot study. J Clin Psychiatry $\underline{63}$, 442-446

Friedel RO (2004): Dopamine Dysfunction in Borderline Personality Disorder: A Hypothesis. Neuropsychopharmacology 29, 1029-1039

Friedel RO, Jackson WT, Huston CS, May RS, Kirby NL, Stoves A (2008): Risperidone treatment of borderline personality disorder assessed by a borderline personality disorder-specific outcome measure: a pilot study. J Clin Psychopharmacol 28,345-347

Gardner DL, Cowdry RW (1985): Alprazolam-induced dyscontrol in borderline personality disorder. Am J Psychiatry 142, 98-100

Gardner DL, Cowdry RW (1986): Positive effects of carbamazepine on behavioral dyscontrol in borderline personality disorder. Am J Psychiatry $\underline{143}, 519-522$

Gescher DM ,Will B, Malevani J ( 2013): Psychosoziale Integration von stationären Patienten mit Borderline-Persönlichkeitsstörung. Nervenarzt 84, 326-333

Gianoli MO, Jane JS, O'Brien E, Ralevski E (2012): Treatment for comorbid borderline personality disorder and alcohol use disorders: a review of the evidence and future recommendations. Exp Clin Psychopharmacol 20, 333-344

Giesen-Bloo J, van Dyck R, Spinhoven P, Tilburg W, Dirksen C, van Asselt T, Kremers I, Nador, M, Arntz A (2006): Outpatient Psychotherapy for Borderline Personality Disorder. Archives of General Psychiatry $\underline{63}$, 649-658

Goldberg SC, Schulz SC, Schulz PM, Resnick RJ, Hamer RM, Friedel RO (1986): Borderline and schizotypal personality disorders treated with low-dose thiothixene vs placebo. Arch Gen Psychiatry $\underline{43}$, 680-686

Griengl H, Dantendorfer K (2001): Naltrexone as a treatment of self-injurious behaviour: a case report. Eur Psychiatry $\underline{16}$, 193-194 
Gunderson JG (1984): Borderline Personality Disorder. American Psychiatric Press. Washington D.C

Gunderson JG: Borderline, Diagnostik, Therapie, Forschung; Verlag Hans Huber, Bern 2005

Gunderson JG, Singer MT (1975): Defining borderline patients:an overview. Amer.J. Psychiat. 132,1-10

Heinze M, Andreae D, Grohmann R (2005): Pharmacotherapy of personality disorders in german speaking countries: state and changes in the last decade. Pharmacopsychiatry $\underline{38}, 201-205$

Helgeland M, Torgersen S (2004): Developmental antecedents of borderline personality disorder. Compr Psychiatry $\underline{45}$, 138-147

Herman JL, Perry C, Kolk B (1989): Childhood trauma in borderline personality disorder. American Journal of Psychiatry 146, 490-495

Herpertz SC, Saß H (Hrsg): Persönlichkeitsstörungen; Thieme, Stuttgart 2002

Herpertz SC und Wenning B: Emotional instabile Persönlichkeitsstörung. In: Herpertz SC, Saß H. (Hrsg) Persönlichkeitsstörungen; Thieme, Stuttgart 2002

Herpertz SC, Saß H (1997): Impulsivität und Impulskontrolle - zur psychologischen und psychopathologischen Konzeptualisierung. Nervenarzt $\underline{68}, 171-183$

Hilger E, Barnas C, Kasper S (2003): Quetiapine in the treatment of borderline personality disorder. World J Biol Psychiatry $\underline{4}$, 42-44

Hollander E, Swann AC, Coccaro EF, Jiang P, Smith TB (2005): Impact of trait impulsivity and state aggression on divalproex versus placebo response in borderline personality disorder. Am J Psychiatry 162, 621-624

Hollander E, Tracy KA, Swann AC, Coccaro EF, McElroy SL, Wozna P, Sommerville KW, Nemeroff CB (2003): Divalproex in the treatment of impulsive aggression: efficacy efficacy in cluster B personality disorders. Neuropsychopharmacology 28, 1186-1197

Jerschke S, Meixner K, Richter H, Bohus M (1998): Zur Behandlungsgeschichte und Versorgungssituation von Patientinnen mit Borderline-Persönlichkeitsstörung in der Bundesrepublik Deutschland. Fortschr Neurol Psychiatr $\underline{66}, 545-552$

Johnson JG, Cohen P, Brown J, Smailes E, Bernstein DP (1999): Childhood maltreatment increases risk for personality disorders during early adulthood. Archives of General Psychiatry $\underline{56}, 600-608$

Katzelnick DJ, Kobak KA, Greist JH, Jefferson JW, Mantle JM, Serlin RC (1995): Sertraline for social phobia: a double-blind, placebo-controlled crossover study. Am J Psychiatry 152, 1368-1371 
Kavoussi RJ, Liu J, Cocarro EF (1994): An open trial of sertraline in personalitydisordered patients with impulsive aggression. J Clin Psychiatry $\underline{55}$, 137-141

Lampe K, Konrad K, Kroener S, Fast K, Kunert HJ, Herpert, SC (2007): Neuropsychological and behavioural disinhibition in adult ADHD compared to borderline personality disorder. Psychological Medicine $\underline{37}, 1717-1729$

Leichsenring F, Leibing E, Kruse J, New AS, Leweke F. (2011) Borderline personality disorder. Lancet. $\underline{377}, 74-84$

Lieb C, Zanarini C, Schmahl C, Linehan MM, Bohus M (2004): Borderline personality disorder. Lancet $\underline{364}, 453-461$

Liebowitz MR, Quitkin FM, Stewart JW, McGrath PJ, Harrison WM, Markowitz JS, Rabkin JG, Tricamo E, Goetz DM, Klein DF (1988): Antidepressant specificity in atypical depression. Arch Gen Psychiatry $\underline{45}$, 129-137

Linehan MM: Cognitive-Behavioral Treatment of Borderline Personality Disorder; Guilfort, New York 1993

Linehan MM: Dialektisch-Behaviorale Therapie der Borderline-PersönlichkeitsStörung; CIP-Medien, München1996

Linehan MM, Heard HI, Armstrong HE (1993): Naturalistic follow-up of a behavioural treatment for chronically parasuicidal borderline patients. Arch Gen Psychiatry $\underline{50}, 971$ 974

Linehan MM, McDavid JD, Brown MZ, Sayrs JH, Gallop RJ (20208): Olanzapine plus dialectical behavior therapy for women with high irritability who meet criteria for borderline personality disorder: a double-blind, placebo-controlled pilot study. J Clin Psychiatry $\underline{69}$, 999-1005

Linehan MM, Comtois KA, Murray AM, Brown MZ, Gallop RJ, Heard HL, Korslund KE, Tutek DA, Reynolds S, Lindenboim N (2006): Two-year randomized controlled trial and follow-up of dialectical behavior therapy vs. Therapy by experts for suicidal behaviors and borderline personality disorder. Archives of General Psychiatry $\underline{63}, 757-766$

Links PS, Steiner M, Offord D R, Eppel A (1988): Characteristics of borderline personality disorder: a Canadian study. Canadian Journal of Psychiatry $\underline{33}$, 336-340

Links $P$ (1992): Psychiatric rehabilitation model for borderline personality disorder. Canadian Journal of Psychiatry $\underline{38}, 35-38$

Linnoila VM, Virkkunen M (1992): Aggression, suicidality and serotonin. Journal of Clinical Psychiatry $\underline{53}, 46-51$

Mann JJ, Malone KM (1997): Cerebrospinal fluid amines and higher lethality suicide attempts in depressed inpatients. Biological Psychiatry $\underline{41}, 162-171$

Markovitz PJ, Calabrese Jr, Schulz SC, Meltzer HY (1991): Fluoxetine in the treatment of borderline and schizotypal personality disorders. Am J Psychiatry 148, 1064-1067 
Markowitz PJ, Wagner SC (1995): Venlafaxine in the treatment of borderline personality disorder. Psychopharmacol Bull 31, 773-777

Mc Gee MD (1997): Cessation of self-mutilation in a patient with borderline personality disorder treated with naltrexone. J Clin Psychiatry $\underline{58}$, 32-33

McGlashan TH (1986): The Chestnut Lodge follow-up study. III. Long-term outcome of borderline personalities. Archives of General Psychiatry $\underline{43}, 20-30$

Mobascher A, Mobascher J, Schlemper V, Winterer G, Malevani J (2006): Aripiprazole pharmacotherapy of borderline personality disorder. Pharmacopsychiatry $\underline{39}, 111-112$

Modestin J, Nussbaumer C, Angst K, Scheidegger P, Hell D (1997): Use of potentially abusive psychotropic substances in psychiatric inpatients. European Archives of Psychiatry and Clinical Neuroscience 247,146-153

Montgomery SA, Montgomery D (1982): Pharmacological prevention of suicidal behaviour. J Affect Disord 4, 291-298

Nickel MK, Loew TH (2008): Treatment of aggression with topiramate in male borderline patients, part II: 18-month follow-up. Eur Psychiatry 23, 115-117

Nickel MK, Loew TH, Pedrosa Gil F (2007): Aripiprazole in treatment of borderline patients, part II: an 18-month follow-up. Psychopharmacology (Berl) 191, 1023-1026

Nickel MK, Nickel C, Mitterlehner FO, Tritt K, Lahmann C, Leiberich PK, Rother WK, Loew TH (2004): Topiramate treatment of aggression in female borderline personality disorder patients: A double-blind, placebo-controlled study. Journal of Clinical Psychiatry $\underline{65}, 1515-1519$

Nickel MK, Muehlbacher M, Nickel C, Kettler C, Pedrosa Gil F, Bachler E, Buschmann W, Rother N, Fartacek R, Egger C, Anvar J, Rother WK, Loew TH, Kaplan P (2006): Aripiprazole in the treatment of patients with borderline personality disorder: a doubleblind, placebo-controlled study. Am J Psychiatry 163, 833-838

Nickel MK, Nickel C, Kaplan P, Lahmann C, Muhlbacher M, Tritt K, Krawczyk J, Leiberich PK, Rother WK, Loew TH (2005): Treatment of aggression with topiramate in male borderline patients: A double-blind, placebo-controlled study. Biological Psychiatry 57, 495-499

Ogata SN, Silk KR, Goodrich S, Lohr NE, Westen D, Hill EM (1990): Childhood sexual and physical abuse in adult patients with borderline personality disorder. American Journal of Psychiatry 147, 1008-13

Pascual JC, Madre M, Soler J, Barrachina J, Campins MJ, Alvarez E, Pérez V (2006): Injectable atypical antipsychotics for agitation in borderline personality disorder. Pharmacopsychiatry $\underline{39}, 117-118$ 
Pascual JC, Soler J, Puigdemont D, Pérez-Egea R, Tiana T, Alvarez E, Pérez V (2008): Ziprasidone in the treatment of borderline personality disorder: a double-blind, placebo-controlled, randomized study. J Clin Psychiatry $\underline{69}$, 603-608

Paris J (2002): Chronic suicidality in borderline personality disorder. Psychiatr Serv $\underline{53}$, 738-42

Paris J (2005): Borderline personality disorder. CMAJ 172, 1579-1583

Paris J, Zweig-Frank H (2001): A 27-year follow-up of patients with borderline personality disorder. Compr Psychiatry $\underline{42}$, 485-487

Paris J, Zweig-Frank H, Guzder J (1994): Risk factors for borderline personality disorder in male outpatients. Journal of Nervous and Mental Disease 182, 375-380

Parsons B, Quitkin FM, McGrath PJ, Stewart JW, Tricamo E, Ocepek-Welikson K, Harrison W, Rabkin JG, Wager SG, Nunes E (1989): Phenelzine, imipramine and placebo in borderline patients meeting criteria for atypical depression. Psychopharmacol Bull 25, 524-534

Perrella C, Carrus D, Costa E, Schifano F (2007): Quetiapine for the treatment of borderline personality disorder; an open-label study. Prog Neuropsychopharmacol Biol Psychiatry $\underline{31}, 158-163$

Pinto OC, Akiskal HS (1998): Lamotrigine as a promising approach to borderline personality: an open case series without concurrent DSM IV major mood disorder. J Affect Disord 52, 333-343

Philipsen A, Schmahl C, Lieb K (2004): Naloxone in the treatment of acute dissociative states in female patients with borderline personality disorder. Pharmacopsychiatry $\underline{37}$, $1-4$

Posner J, Russell JA, Peterson BS (2005): The circumplex model of affect: an integrative approach to affective neuroscience, cognitive development, and psychopathology. Devepomental Psychopathology 17, 715-734

Resch F: Entwicklungspsychopathologie des Kindes- und Jugendalters; Beltz Verlag, Weinheim 1996

Remmel A, Bohus M (2006): Pharmakologische und psychotherapeutische Behandlung der Borderline-Störung. Zeitschrift für Psychiatrie, Psychotherapie und Psychologie 54, 185-197

Rifkin A, Quitkin F, Carrillo C, Blumberg AG, Klein DF (1972): Lithium carbonate in emotionally unstable character disorders. Arch Gen Psychiatry 27, 519-523

Rinne T, van den Brink W, Wouters L, van Dyck R (2002): SSRI treatment of borderline personality disorder: a randomized, placebo-controlled trial for female patients with bordeline personality disorder. Am J Psychiatry 159, 2048-2054 
Ripoll LH, Triebwasser J, Siever LJ. Evidence-based pharmacotherapy for personality disorders. Int J Neuropsychopharmacol. 2011 Oct 14, 1257-88

Rizvi ST (2002): Lamotrigine and borderline personality disorder. J Child Adolesc Psychopharmacol 12, 365-366

Rocca P, Marchiaro L, Cocuzza E, Bogetto F J (2002): Treatment of borderline personality disorder with risperidone. Clin Psychiatry $\underline{63}(3), 241-244$

Roy A, Linnoila M (1988): Suicidal behavior, impulsiveness and serotonin. Acta Psychiatrica Scandinavica $\underline{78}, 529-535$

Salzman S, Wolfson AN, Schatzberg A, Looper J, Henke R, Albanese M, Schwartz J, Miyawaki E (1995): Effect of fluoxetine on anger in symptomatic volunteers with borderline personality disorder. J Clin Psychopharmacol 15, 23-29

Samuels J, Eaton WW, Bienvenu OJ, Brown CH, Costa PT, Nestadt G (2002): Prevalence and correlates of personality disorders in a community sample. $\mathrm{Br} \mathrm{J}$ Psychiatry $\underline{180}, 536-542$

$\mathrm{Sa}$ H: Persönlichkeitsstörungen. In: Psychiatrie der Gegenwart. Band 6. Hrsg. v. Helmchen H, Henn F, Lauter H, Sartorius N, 4. Auflage; Springer-Verlag, Berlin, Heidelberg, New York 2000, 275-330

Saß H, Jünemann K, Herpertz SC: Historischer Rückblick. In: Herpertz SC, Saß H (Hrsg): Persönlichkeitsstörungen. Thieme, Stuttgart 2002, 1-3

Schmahl C, Bohus M (2001): Symptomorientierte Pharmakotherapie bei BorderlinePersönlichkeitsstörungen. Fortschr Neurol Psychiatr $\underline{69}$, 310-321

Schmahl CG, Stiglmayr CE, Böhme R, Bohus M (1999): Behandlung von dissoziativen Symptomen bei Borderline-Persönlichkeitsstörungen mit Naltrexon. Nervenarzt, $\underline{70}$, 262-264

Schmitz B, Fydrich T, Limbacher K: Persönlichkeitsstörungen: Diagnostik und Psychotherapie; Beltz Psychologie Verlags Union, Weinheim 1996

Schulz SC, Camlin KL, Berry SA, Jesberger JA (1999): Olanzapine safety and efficacy in patients with borderline personality disorder and comorbid dysthymia. Biol Psychiatry $\underline{46}$, 1429-1435

Sheard MH, Marini JL, Bridges Cl, Wagner E (1976): The effect of lithium on unipolar aggressive behaviour in man. Am J Psychiatry 133, 1409-1413

Silverman JM, Pinkham L, Horvath TB, Coccaro EF, Klar H, Schear S, Apter S, Davidson M, Mohs RC, Siever LJ (1991): Affective and impulsive personality disorder traits in the relatives of patients with borderline personality disorder. American Journal of Psychiatry $148,1378-1385$ 
Skodol AE, Buckley P, Charles E (1983): Is there a characteristic pattern to the treatment history of outpatients with borderline personality? J Nerv Ment Dis $\underline{171}$, 405410

Skodol AE, Siever LJ, Livesley WJ, Gunderson JG, Pfohl B, Widinger TA (2002): biology, genetics and clinical course. Biol Psychiatry $\underline{51}$, 951-963.

Skodol AE, Stout RL, McGlashan TH, Grilo CM, Gunderson JG, Shea MT, Morey LC, Zanarini MC, Dyck IR, Oldham JM (1999): Co-occurrence of mood and personality disorders: a report from the Collaborative Longitudinal Personality Disorders Study (CLPS). Depress. Anxiety 10, 175-182

Soler J, Pascual JC, Campins J, Barrachina J, Puigdemont D, Alvarez E, Pérez V (2005): Double-blind, placebo-controlled study of dialectical behavior therapy plus olanzapine for borderline personality disorder. Am J Psychiatry 162, 1221-1224

Soloff PH (1998): Algorithms for pharmacological treatment of personality dimensions: symptom-specific treatments for cognitive-perceptual, affective, and impulsivebehavioural dysregulation. Bull Menninger Clin $\underline{62}, 195-214$

Soloff PH (2000): Psychopharmacology of borderline personality disorder. Psych Clin North Am 23, 169-192

Soloff PH, George A, Nathan S (1989): Amitriptyline versus haloperidol in borderlines: final outcomes and predictors of response. J Clin Psychopharmacol $\underline{9}$, 238-246

Soloff PH, George A, Nathan RS, Schulz PM, Perel JM (1986): Paradoxical effects of amitriptyline in borderline patients. American Journal of Psychiatry 143, 1603-1605

Soloff PH, George A, Nathan RS, Schul PM, Ulrich RF, Perel JM (1986): Progress in pharmacotherapy of borderline disorders: A double-blind study of amitriptyline, haloperidol, and placebo. Arch Gen Psychiatry 43, 691-697

Soloff PH, Cornelius J, George A, Nathan S, Perel JM, Ulrich RF (1993): Efficacy of phenelzine and haloperidol in borderline personality disorder. Arch Gen Psychiatry $\underline{50}$, 377-385

Sonne S, Rubey R, Brady K, Malcolm R, Morris T (1996): Naltrexone treatment of selfinjurious thoughts and behaviours. J Nerv Ment Dis $\underline{184}, 192-195$

Stein G (1992): Drug treatment of the personality disorders. Br J Psychiatry 161, 167184

Stein DJ, Simeon D, Frenkel M, Islam MN, Hollander E (1995): An open trial of valproate in borderline personality disorder. J Clin Psychiatry $\underline{56}, 506$

Stiglmayr C, Shapior DA, Stieglitz RD, Limberger MF, Bohus M (2001). Experience of aversive tension and dissociation in female patients with borderline personality disorder - a controlled study. Journal of Psychiatric Research $\underline{35}$, 111- 118 
Stone, MH: Long-term outcome in personality disorder. In: Tyrer, P, Stein G (Eds): Personality Disorder Reviewed. Caskell/Royal College of Psychiatrists, London 1993, 321-345

Swartz MS, Blazer D, George L, Winfield I (1990): Estimating the prevalence of borderline personality disorder in the community. J Personal Disord 4 , 257-72

Szigethy EM, Schulz SC (1997): Risperidone in comorbid borderline personality disorder and dysthymia. J Clin Psychopharmacol 17, 326-327

Teicher MH, Andersen SL, Polcari A, Anderson CM, Navalta CM (2002): Developmental neurobioloy of childhood stress and trauma. Psychiatric Clinic of North America 25, 397-426

Torgersen S (1994): Genetics in Borderline Conditions. Acta Psychiatrica Scandinavian supplementum $\underline{379}, 19-25$

Torgersen S, Lygren S, Oien PA, Skre I, Onstad S, Edvardsen J, Tambs K,Kringlen E (2000): A twin study of personality disorders. Compr Psychiatry 41, 416-425

Tritt K, Nickel C, Lahmann C, Leiberich PK, Rother W, Loew TH, Nickel MK (2005): Lamotrigine treatment of aggression in female borderline-patients: A randomized, double-blind, placebo-controlled study. Journal of Psychopharmacology 19, 287-291

Van den Eynde F, Senturk V, Naudts K, Vogels C, Bernagie K, Thas O, van Heeringen C, Audenaert K (2008): Efficacy of quetiapine for impulsivity and affective symptoms in borderline personality disorder. J Clin Psychopharmacol 28, 147-155

Van den Eynde F, Senturk V, Naudts K, Vogels C, Bernagie K, Thas O, van Heeringen C, Audenaert K (2008): Efficacy of quetiapine for impulsivity and affective symptoms in borderline personality disorder. J Clin Psychopharmacol $\underline{28}$, 147-155

van Vliet IM, den Boer JA, Westenberg HG (1994): Psychopharmacological treatment of social phobia; a double blind placebo controlled study with fluvoxamine. Psychopharmacology (Berl) 115, 128-134

Wedekind D, Bandelow B, Rüther E (2005): Pharmakotherapie bei Persönlichkeitsstörungen. Fortschr Neurol Psychiatr $\underline{73}$, 259-267

WHO (Weltgesundheitsorganisation): Internationale Klassifikation psychischer Störungen: ICD-10, Kapitel V (F); klinisch-diagnostische Leitlinien. 2. Auflage; WHO, Bern, Göttingen, Toronto, Seattle 1993.

Widiger TA, Weissmann MM (1991): Epidemiology of borderline personality disorder. Hosp Community Psychiatry $\underline{42}$, 1015-1021

Wilcox JA (1995): Divalproex sodium as a treatment for borderline personality disorder. Ann Clin Psychiatry $\underline{7}$, 33-37

Winkler M, Rossi P (2001): Borderline-Persönlichkeitsstörung und Aufmerksamkeitsdefizit / Hyperaktivitätsstörung bei Erwachsenen. Persönlichkeitsstörungen $\underline{5}$, 39-48 
Young JE, Swift W (1988): Schema-focused cognitive therapy for personality disorder; part 1. International Cognitive Therapy Newsletter $\underline{4}, 13-14$

Young JE, Klosko JS, Weishaar ME: Schematherapie. Ein praxisorientiertes Handbuch. Junfermann Verlag, Paderborn 2005

Zanarini MC (2004): Update on pharmacotherapy of borderline personality disorder. Curr Psychiatry Rep $\underline{6}, 66-70$

Zanarini MC, Frankenburg FR (2001): Olanzapine treatment of female borderline personality disorder patients: a double-blind, placebo-controlled pilot study. J Clin Psychiatry $\underline{62}, 849-854$

Zanarini MC, Frankenburg FR, Parachini EA (2004): A preliminary, randomized trial of fluoxetine, olanzapine, and the olanzapine-fluoxetine combination in woman with borderline personality disorder. J Clin Psychiatry $\underline{65}, 903-907$

Zanarini MC, Frankenburg FR, Khera GS, Bleichmar J (2001): Treatment histories of borderline inpatients. Compr Psychiatry 42, 144-150

Zanarini MC, Frankenburg FR, Hennen J, Silk KR (2003): The longitudinal course of borderline psychopathology: 6-year prospective follow-up of the phenomenology of borderline personality disorder. Am J Psychiatry 160, 274-283

Zanarini MC, Frankenburg FR, Hennen J, Silk KR (2004): Mental health utilization by borderline personality disorder patients and axis II comparison subjects followed prospectively for 6 years. J Clin Psychiatry $\underline{65}, 28-36$

Zanarini MC, Frankenburg FR, Hennen J, Reich DB, Silk KR (2004): Axis I comorbidity in patients with borderline personlity disorder: 6-year follow-up and prediction of time to remission. Am J Psychiatry 161, 2108-2114

Zanarini MC, Frances R, Frankenburg MD, Dubo E, Sickel M, Trikha A, Levin A, Reynolds V (1998): Axis I comorbidity of borderline personality disorder. Am J Psychiatry 155, 1733-1739

Zanarini MC, Williams AA, Lewis RE, Reich RB, Vera SC, Marino MF, Levin A, Yong L, Frankenburg FR (1997): Reported pathological childhood experiences associated with the development of borderline personality disorder. American Journal of Psychiatry $\underline{154}, 1101-1106$

Zelkowitz P, Paris J, Guzder J, Feldman R (2001): Diathesis and stressors in borderline pathology of childhood: The role of neuropsychological risk and trauma. $\mathrm{J}$

Am Acad Child Adolesc Psychiatry 40, 100 -105

Zimmermann M, Mattia JI (1999): Axis I diagnostic comorbidity and borderline personality disorder. Compr Psychiatry $\underline{40}$, 245-252

Zittel Conklin C, Westen D (2005): Borderline personality disorder in clinical practice. Am J Psychiatry 162, 867-875 


\section{Danksagung}

Mein besonderer Dank geht an PD Dr. Wedekind für seine kompetente, geduldige und engagierte Betreuung und Förderung.

Danken möchte ich für das entgegengebrachte Vertrauen. Durch zahlreiche fruchtbare Gespräche und durch das Anvertrauen von wichtigen Aufgaben hat er wesentlich zu meiner Förderung und Entwicklung beigetragen. 